ANHANG 


\title{
Anlage 1
}

\section{Reichs- und Preußisches Justizministerium Geschäftsverteilungsplan Stand vom 22. Oktober 1934}

\author{
Akten des Reichsfinanzministeriums \\ Bundesarchiv, Sign. $R$ 2/23901
}

\author{
Minister: Dr. h. c. Gürtner \\ Ministerbüro: Dr. v. Dohnanyi, ORR. (s. auch Abt. II) \\ Kaulbach, LGR. (s. auch Abt. V). \\ Staatssekretäre: \\ Dr. Freisler, Preuß. Staatsrat, M.d.R.
}

Dr. Dr. h.c. Schlegelberger

Zur persönlichen Verfügung:

Dr. Dr. Wilke, OLGR. (s. auch Abt. IV)

Dr. Heintzeler, AR. (s. auch ZS)
Haastert, OLGR. (s. auch Abt. ZF u. I)

Dr. Ebert, StAR. (s. auch Abt. II)

\section{Geschäftsbereich}

Zentralabteilung S

Abteilung I (Personalsachen)

- Näheres s. unten -

Abteilung IV (Bürgerl. Recht)

Abteilung V (Handelsrecht, öff. Recht)

Abteilung LA (Familiengüter)

\section{Zentralabteilung $\mathrm{F}$}

Abteilung I (Personalsachen)

- Näheres s. unten -

Abteilung II (Strafgesetzgebung)

Abteilung III (Strafrechtspflege)

Abteilung VI (Preuß. Justizverwaltungssachen)

Abteilung RJP. (Ausbildung)

Zuständigkeit in Personalsachen:

a) Reichsjustizministerium

b) Reichsgericht, Reichsanwaltschaft und Reichspatentamt; soweit es sich um leitende Beamte handelt, unter Mitzeichnung von StS. Dr. Freisler.

c) Gerichte u. Staatsanwaltschaften in den Bezirken: Düsseldorf, Frankfurt a. M. (mit Sigmaringen), Hamm, Köln, Königsberg, Marienwerder u. Naumburg (mit Jena) - leitende Beamte s. Abschnitt $\mathrm{c}$ bei StS. Dr. Freisler,

d) Patentanwälte. a) Preußisches Justizministerium und Landesamt für Familiengüter

b) Reichs-Justizprüfungsamt, Volksgerichtshof und Reichserbhofgericht; soweit es sich um leitende Beamte handelt, unter Mitzeichnung von StS. Dr. Schlegelberger.

c) Gerichte, Staatsanwaltschaften u. Gemeinschaftslager Hanns Kerrl:

A: leitende Beamte aller Behörden (unter Mitzeichnung von StS. Dr. Schlegelberger),

B: im übrigen die Bezirke Berlin, Breslau, Celle, Kassel, Kiel u. Stettin.

d) Strafvollzugsbehörden

e) Rechtsanwälte und Notare. 


\begin{tabular}{|c|c|c|}
\hline \multicolumn{2}{|c|}{ Arbeitsgebiete } & \multirow{2}{*}{$\begin{array}{l}\text { Vertreter } \\
\text { Richter, MR }\end{array}$} \\
\hline & $\begin{array}{l}\text { Dr. Doerner, ORR. (s. auch ZF 5) } \\
\text { Presse- u. Rundfunksachen (Presse- u. Rundfunkrecht } \\
\text { s. Abt. V). }\end{array}$ & \\
\hline 2. & $\begin{array}{l}\text { Wagner, } M R \text {. (s. auch Ab. I) } \\
\text { 1) Personalien des höheren Dienstes im RJM. } \\
\text { 2) Verwaltung des RJM. und Korref. für Ref. } 3 \\
\text { 3) Reichshaushalts- u. -verwaltungssachen } \\
\text { 4) Beschwerden über Personalentscheidungen der nichtpreu- } \\
\text { Bischen Länder. }\end{array}$ & Sauer \\
\hline & $\begin{array}{l}\text { Dr. Heintzeler, } A R \text {. (s. auch StS. Schlegelberger) } \\
\text { Sonderaufträge aus Ref. } 2 \text {. }\end{array}$ & - \\
\hline & $\begin{array}{l}\text { Westfeld, MBD. (s. auch Abt. I) } \\
\text { 1) Verwaltungsangelegenheiten Voßstr. } 5 . \\
\text { 2) Geschäftsgang des Ministeriums } \\
\text { 3) Kassenaufsicht über die Zahlstelle des RJM. } \\
\text { 4) Sonderaufträge aus Ref. } 2 \text {. }\end{array}$ & Böhme, MA. \\
\hline & $\begin{array}{l}\text { Koffka, MR. (s. auch Abt. V) } \\
\text { Bücherei des RJM. }\end{array}$ & Pätzold \\
\hline & $\begin{array}{l}\text { Dr. Pätzold, ORR. (s. auch Abt. IV) } \\
\text { Schriftleitung der Deutschen Justiz zus. mit ZF. }\end{array}$ & Kühnemann \\
\hline & $\begin{array}{l}\text { Dr. Sauer, MR. } \\
\text { 1) Angelegenheiten der Verreichlichung der Justiz, soweit } \\
\text { nicht die Entwürfe in den anderen Abteilungen ausgearbei- } \\
\text { tet werden, in Benehmen mit OLGR. Haastert (ZF 4) } \\
\text { 2) Justizhaushalts- u. - Verwaltungssachen der nichtpreußi- } \\
\text { schen Länder. }\end{array}$ & Hauptvogel \\
\hline & $\begin{array}{l}\text { Dr. Schröder, } L R . \\
\text { Vorbearb. im Ref. } 6 \text { und Sonderaufträge. }\end{array}$ & - \\
\hline
\end{tabular}

Zentralabteilung des StS. Dr. Freisler $(=Z F)$

\begin{tabular}{|c|c|c|}
\hline \multicolumn{2}{|c|}{ Arbeitsgebiete } & \multirow{2}{*}{$\begin{array}{l}\text { Vertreter } \\
\text { s. Abt. I }\end{array}$} \\
\hline 1. & $\begin{array}{l}\text { Dr. Nadler, Min.Dir. (s. auch Abt. I) } \\
\text { Personalsachen des höheren Dienstes im Pr.JM. }\end{array}$ & \\
\hline 2. & $\begin{array}{l}\text { Lutterloh, MR. (s. auch Abt. I) u. Dr. Krug, OStA. } \\
\text { (s. auch Abt. III) } \\
\text { Deutsche Justiz zus. mit ZS. }\end{array}$ & Wittland, Rempe \\
\hline 3. & $\begin{array}{l}\text { Kunisch, MR. (s. auch Abt. I) } \\
\text { Gemeinschaftslager, Personalsachen unter Beteiligung der } \\
\text { Bezirksreferenten. }\end{array}$ & Friedrich \\
\hline & $\begin{array}{l}\text { Haastert, OLGR. (s. auch StS. Freisler u. Abt. I) } \\
\text { Angelegenheiten der Verreichlichung der Justiz, soweit nicht } \\
\text { die Entwürfe in den anderen Abteilungen ausgearbeitet wer- } \\
\text { den, im Benehmen mit MR. Sauer (ZS 6). }\end{array}$ & - \\
\hline
\end{tabular}


5. Dr. Doerner, ORR. (s. auch ZS 1)

Richter, MR.

Presse- u. Rundfunksachen (Presse- u. Rundfunktrecht s. Abt. V).

6. MinVerwDir., m. Wahrnehmung d. Gesch. beauftr.:

Urlacher

Bender, ORR. (s. auch Abt. I)

1) Zeichnung der Anweisungen über sächliche Ausgaben für das Pr.JM. bis zum Betrage von 1000 RM.

2) Anweisung der Reisekostenvorschüsse in preuß. Angelegenheiten

3) Anschaffungen für das Gebäude und die Ministerwohnung Wilhelmstr., soweit nicht die Kosten aus dem Baufonds zu decken sind.

7. Dr. Wittland, KGR. (s. auch Abt. I)

Lutterloh

Bücherei des Pr JM.

8. Dr. Mitzschke, LGR. (s. auch Abt. IV)

Volks-Rechtsschutz.

9. Friedrich, $A G R$. (s. auch Abt. VI u. RJP.)

Vorbearb. für das Ref. Kunisch.

\section{Zentralstaatsanwaltschaft}

10. Dr. Joël, StAR.

von Haacke

1) Angelegenheiten der Zentralstaatsanwaltschaft,

2) außerpreuß. Sonderfälle.

11. von Haacke, StAR.

1) Angelegenheiten der Zentralstaatsanwaltschaft,

2) außerpreuß. Sonderfälle.

12. Meyer, $G A$.

Vorbearb. in den Angelegenheiten der Zentralstaatsanwaltschaft.

\section{Vorbemerkung für alle Abteilungen}

Fragen des ausländischen u. des internationalen Rechts werden von derjenigen Stelle bearbeitet, der das einschlägige heimische Rechtsgebiet zugewiesen ist, sofern der Verteilungsplan nichts anderes bemerkt.

\section{Abteilung I}

(Personalsachen und Gerichtsorganisation)

\section{Leiter: Ministerialdirektor Dr. Nadler}

1. Leitung der Abteilung; die Einzelsachen, die die Besetzung der preuß. Behörden mit Hilfskräften aller Art, das Besoldungswesen oder die Zubilligung von Sondervergütungen betreffen, zeichnet der Leiter der Abt. VI.

2. Bearbeitung der Stellenbesetzung von Besoldungsgruppe $A l$ a (preuß.) aufwärts und Mitwirkung bei der Besetzung des Reichsjustizprüfungsamts (s. RJP Ref. 4).

Vertreter: Lutterloh, MR. 
1. Lutterloh, MR. (s. auch ZF)

1) Personalien der Richter $u$. Verwaltungssachen aus

1.
a) Köln,
b) Frankfurt a. M.
c) Hamm
d) aus dem Geschäftsbereich der früheren Friedens- abteilung West- u. Ostreferat.

2) Dolmetscher.

2. Dr. Doerffler, MR. (s. auch Abt. II)

Personalien der Staatsanwälte u. Verwaltungssachen der Staatsanwaltschaften.

3. Kunisch, MR. (s. auch ZF)

Personalien u. sonstige Angelegenheiten der Rechtsanwälte u. Notare (Gesetzgeb. s. Abt. IV).

4. Wagner, MR. (s. auch ZS)

1) Personalien aller Dienstzweige u. Verwaltungssachen
a) des Reichsgerichts,
b) des Volksgerichtshofs,
c) der Reichsanwaltschaft,
d) des Reichspatentamts,
e) des Reichserbhofgerichts.

2) Korref. für Personalsachen des mittleren u. AmtsgehilfenDienstes im RJM. (Ref. Westfeld).

5. Dr. Wittland, KGR. (s. auch ZF)

1) Organisationssachen;

2) Unwiderrufliche Anstellung der Gerichtsassessoren;

3) Dienststrafrecht u. Dienststrafgerichte (Gesetzgebung s. Abt. V);

4) Angelegenheiten betr. Anwendung der Sozialversicherungsgesetze auf Beamte, Angestellte u. Arbeiter;

5) Personalien der Richter $u$. Verwaltungssachen aus
a) Celle,
b) Kassel,
c) Kiel,
d) Landeserbhofgericht, zus. mit ErbhofGR. Dr. Hopp (s. Ref. 8).

6. Haastert, OLGR. (s. auch StS. Freisler u. ZF)

1) Personalien der Richter u. Verwaltungssachen aus
a) Breslau,
b) Düsseldorf;

2) Allgem. Beamtenangelegenheiten (Gesetzgebung s. Abt. V);

3) Allgem. Angelegenheiten der Beamten des höheren Dienstes;

4) Sonderaufträge.

7. Grußendorf, $L G D$.

Personalien der Richter $u$. Verwaltungssachen aus
a) Berlin
b) Königsberg,
c) Marienwerder,
d) Naumburg u. gemeinschaftl. Gerichte,
e) Stettin.
a) Haastert
b) Haastert
c) Grußendorf
d) Grußendorf

2. Willers

Wittland

Wittland

Sauer

(Doerffler)

1. Lutterloh

2. Grußendorf

3,4 . Haastert

5. a-c) Doerffler

d) Hopp

1.

a) Grußendorf

b) Lutterloh

2, 3 .

Wittland
a) Luttleroh
b) Wittland
c) Wittland
d) Haastert
e) Haastert 


\begin{tabular}{l} 
Arbeitsgebiete \\
\hline 8. $\quad$ Dr. Hopp, ErbhofGR. (s. auch Abt. II) \\
Personalien der Richter u. Verwaltungssachen des Landes- \\
erbhofgerichts, zus. mit KGR. Dr. Wittland (s. Ref. 5).
\end{tabular}

Vertreter

9. Lentz, MR. (s. auch Abt. IV, RJP u. LA).

1) Allgem. u. Einzelsachen der Amtsanwälte einschl. der 1. Willers örtlichen Sitzungsvertreter;

2) Unterstützungswesen einschl. der Zeichnung im Rahmen 2. Willers der preuß. Verfügung vom 25.6.30-ZB. 1141 -.

3) Einzelsachen der Beamten im Büro-, Kanzlei- u. Wacht- 3. Osburg meisterdienst aus
a) Kiel,
b) Königsberg,
c) Marienwerder,
d) Naumburg u. gemeinschaftl. Gerichte,
e) aus dem Geschäftsbereich der früheren Friedens- abteilung Ostreferat.

4) Personalien der Gerichtsvollzieher u. Gerichtskassenvollzieher $u$. Einzelangelegenheiten des Gerichtsvollzieher-

u. Gerichtskassenvollzieherwesens.

10. Willers, MR. (s. auch RJP.)

1) Allgem. Angelegenheiten

a) des oberen, mittleren $u$. unteren Justizdienstes

b) der Angestellten u. Lohnempfänger.

Wittland

Einzelsachen der Beamten im Büro-, Kanzlei- u. Wachtmeisterdienst aus
a) Berlin,
b) Celle

3) Korref. für Ref. 14 (Gundlach) zu 1 und 2.

11. Bender, ORR. (s. auch ZF)

Urlacher

Personalsachen des oberen, mittleren u. Amtsgehilfendienstes, der Angestellten u. der Arbeiter des Pr. JM. einschl. Unterstützungen $u$. Notstandsbeihilfen (Zeichnung im bisherigen Umfange).

12. Westfeld, $M B D$. (s. auch ZS)

Personalsachen des schwierigen u. einfachen mittleren sowie des Amtsgehilfen-Dienstes, der Angestellten u. der Arbeiter des RJM. einschl. Unterstützungen u. Notstandsbeihilfen (Zeichnung im bisherigen Umfange) Korref. Wagner.

13. Osburg, ORR. (s. auch Abt. VI)

Einzelsachen der Beamten im Büro-, Kanzlei- u. Wachtmeisterdienst aus
a) Breslau,
b) Düsseldorf,
c) Hamm,
d) Stettin,
e) aus dem Geschäftsbereich der früheren Friedensabteilung Westreferat.

1.
a) Lentz
b) Gundlach
2. Gundlach 


\section{Arbeitsgebiete}

Vertreter

14. Gundlach, ARat. (s. auch Abt. VI)

1) Allgem. u. Einzelsachen der Versorgungsanwärter;

$1,2,4$. Willers

2) Einzelsachen der Angestellten u. Lohnempfänger;

3) Vorbearb. im Ref. Willers zu 1.;

4) Einzelsachen der Beamten im Büro-, Kanzlei- u. Wachtmeisterdienst aus

a) Frankfurt a.M.,

b) Kassel,

c) Köln.

\section{Abteilung II}

Strafgesetzgebung (Strafrecht, Strafprozeß, Strafvollzug, Auslieferungsrecht, Zwischenstaatliche Rechtshilfe in Strafsachen), bäuerliches Recht

Leiter: Ministerialdirektor Schäfer

Vertreter: Dr. Schäfer, GRR.

Vertreter

1. Dr. Schäfer, GRR.

Rietzsch

1) Generalreferat für die Strafrechtsreform

2) Strafgesetzbuch (laufende Fragen)

3) Strafregisterwesen

4) Geschlechtskrankheitengesetz.

2. Dr. Mettgenberg, MR. (s. auch Abt. III)

Zwischenstaatliches Strafrecht, Auslieferungsrecht u. sonstige

Richter, MR.

zwischenstaatl. Rechtshilfe in Strafsachen (soweit Maßnahmen im Wege der Gesetzgebung od. generelle zwischenstaatl. Verträge in Frage kommen).

3. Richter, $M R$.

1) Generalreferat für die Reform des Nebenstrafrechts (Einführungsgesetz zum Strafgesetzbuch)

2) Amnestiegesetzgebung.

4. Rietzsch, $M R$.

1) Mitarbeit an der Strafrechtsreform

2) Preußisches Strafrecht und sonstiges Landesstrafrecht

3) Leitung der der Abteilung zugewiesenen Assessoren im allgemeinen

4) Sonderaufträge.

5. Dr. Dörffler, MR. (s. auch Abt. I)

1) Mitarbeit an der Strafprozeßreform

2) Gerichtsverfassungsgesetz (laufende Fragen)

3) Verreichlichung auf dem Gebiete des Strafprozeßrechts, soweit Maßnahmen im Wege der Gesetzgebung oder der Verordnung in Frage kommen.

6. Dr. Lehmann, MR.

1) Mitarbeit an der Strafprozeßreform

Schäfer, OLGR.

2) Strafprozeßordnung (laufende Fragen)

3) Militärstrafgerichtsordnung. 
4) Angelegenheiten der Internat. Strafrechts- und Gefängnis-

4. Hauptvogel kommission (Fragen des Strafrechts u. Strafprozesses), insbes. Vorbereitung des Berliner Kongresses $I=X I$. Internationaler Strafrechts- und Gefängniskongreß in Berlin vom 18. bis 24. August 1935].

7. Hauptvogel, $M R$.

1) Strafvollzugsreform (Entwurf des Strafvollzugsgesetzes, 1,2,4. Schafheutle Verreichlichung des Strafvollzugs in Verbindung mit Abt. III)

2) Jugendgerichtsgesetz

3) Angelegenheiten der Internat. Strafrechts- u. Gefängniskommission (Fragen des Strafvollzugs)

4) Kriminal-u. Gefängnisstatistik.

8. Grau, Vizepräs.

1) Mitarbeit an der Strafrechtsreform

2) Militärstrafrecht

3) Sonderaufträge.

9. Dr. von Dohnanyi, ORR. (s. auch MB)

1) Mitarbeit an der Strafrechtsreform

2) Sonderaufträge.

10. Dr. Schäfer, OLGR.

1) Mitarbeit an der Strafrechtsreform

2) Mitarbeit an der Reform des Nebenstrafrechts (Einführungsgesetz zum Strafgesetzbuch)

3) die strafrechtlichen Nebengesetze des Reichs.

11. Dr. Schafheutle, RR.

1) Mitarbeit an der Strafprozeßreform (Ref. Dörffer zu 1 u. 3)

2) Gebühren- und Kostengesetze.

12. Dr. Ebert, StAR. (s. auch StS. Freisler)

1) Mitarbeit an der Strafprozeßreform (Ref. Lehmann zu 1 u. 3)

2) Sonderaufträge.

13. Dr. Ficker, $L G R$.

1) Mitarbeit an der Strafprozeßreform

2) Mitarbeit an der Reform des Nebenstrafrechts

3) ausländisches Schrifttum auf dem Gebiet des Strafrechts, Strafprozesses und Strafvollzugs.

13a. Dr. Meeske, LR.

Vorbearb. für die Reform des Nebenstrafrechts

14. Dr. Vogels, MR. (s. auch Abt. IV)

1) Erbhofrecht (ohne die im Ref. Hopp zu 3 u. 4 bezeichneten Angelegenheiten)

2) Ländliche Entschuldung.

15. Dr. Hopp, ErbhofGR. (s. auch Abt. I)

1) Ländliche Siedlung u. Rentengutsgesetzgebung

2) Mitarbeit in Erbhofsachen nach bes. Zuteilung

3) Beobachtung u. Auswertung der Rechtsprechung der Anerbengerichte

4) Statistik des Erbhofrechts

5) Mitarbeit in Sachen der ländlichen Entschuldung nach bes. Zuteilung.

Schäfer, OLGR.

v. Dohnanyi

Ficker

Ficker

Ebert

Schafheutle

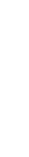

Hopp

Vogels$$
\text { Hopp }
$$

v. Dohnanyi

Schäfer, OLGR. 


\section{Abteilung III \\ (Strafrechtspflege, Strafvollzugsverwaltung)}

Leiter: Ministerialdirektor Dr. Crohne

Zeichnung u. Gegenzeich. der Verfg. in

1) Generalsachen im Strafrecht, Strafprozeß u. Strafregisterwesen, soweit nicht Abt. II zuständig

2) Einzelsachen, soweit sie von politischer, grundsätzlicher od. sonstiger besonderer Bedeutung sind.

Vertreter: Dr. Mettgenberg, MR., in Einzelsachen zu 2 der jeweilige Referent.

\begin{tabular}{|c|c|c|}
\hline \multicolumn{2}{|c|}{ Arbeitsgebiete } & \multirow[t]{2}{*}{ Vertreter } \\
\hline 1. Dr. $M$ & Mettgenberg, MR. (s. auch Abt. II) & \\
\hline & $\begin{array}{l}\text { Auslieferungsrecht u. zwischenstaatliche Rechtshilfe in } \\
\text { Strafsachen (ohne Gesetzgebung u. generelle zwischen- } \\
\text { staatliche Verträge - s. Abt. II) }\end{array}$ & 1. Kutzner \\
\hline 2) & $\begin{array}{l}\text { Mitzeichnung in Einzelfällen in Auslieferungs- u. sonsti- } \\
\text { gen Rechtshilfesachen von grundsätzl. Bedeutung (Ref. } \\
\text { Kutzner) }\end{array}$ & 2.- \\
\hline & $\begin{array}{l}\text { Generalreferat für Strafrecht, Strafprozeß u. Strafregister- } \\
\text { wesen, soweit nicht Abt. II zuständig }\end{array}$ & 3. Pritsch \\
\hline 4) & $\begin{array}{l}\text { Einzelsachen, die die Einholung eines Strafantrags des } \\
\text { Führers u. Reichskanzlers od. eines Reichsministers betref- } \\
\text { fen (in preuß. Sachen, soweit sie von den Ref. für pol. } \\
\text { Strafsachen abgegeben werden). }\end{array}$ & 4. Wille \\
\hline
\end{tabular}

1a. Dr. Stolzenburg, LGR. (s. auch Ref. 3a)

1) Mitarbeit im Generalref. für Strafrecht, Strafprozeß u. Strafregisterwesen

2) Einzelstatistiken

3) Kontrolle der Straf rechtsprechung über grundsätzliche Fragen.

1b. Dr. Reisner, GAss. (vgl. auch Ref. 7b)

Vorbearb. für Ref. Mettgenberg zu Nr.1.

2 u. 3. Mettgenberg

1c. Heitmann, GAss.

Sammlung der Justizverwaltungsbestimmungen auf dem Gebiete des Strafrechts u. Strafprozesses nach Anleitung von Dr. Stolzenburg.

2. Dr. Pritsch, MR.

1) Nichtpol. Einzelstrafsachen aus

1.
a) Berlin,
d) Königsberg,
b) Breslau,
e) Marienwerder,
a) Busch
c) Kiel,
f) Naumburg
b, e) Krug
c, d, f) Kutzner

2) Einzelsachen betr. Entschädigung wegen unschuldig erlit- 2. Busch tener Haft

3) Verfügungen hinsichtlich der zusammengestellten Gnaden- 3. Busch tabellen

4) Dienstaufsichtsbeschwerden gegen Reichsgericht, Volksgerichtshof $u$. Oberreichsanwalt in Strafsachen.

2a. Altmeyer, StAR.

Mitarbeit in nichtpol. Einzelstrafsachen aus dem LGBezirk

Berlin.

4. Busch

Rempe 
2b. Hoffmann, LGR.

Mitarbeit in nichtpol. Einzelstrafsachen aus
a) Breslau
a) Ref. $2 \mathrm{c}$
b) Marienwerder.
b) Ref. 2d

2c. Dr. von Grolman, $L G R$.

Mitarbeit in nichtpol. Einzelstrafsachen aus
a) Königsberg
a) Stolzenburg
b) Naumburg.
b) Altmeyer

2d. Dr. Kramer StAR. (s. auch Ref. 3e)

Mitarbeit in nichtpol. Einzelstrafsachen aus
a) KGBezirk (ohne LG. Berlin)
b) Kiel.
a) Rindermann
b) Altmeyer

2e. Stampe, $R R$.

1) Mitarbeit in Einzelsachen betr. Entschädigung wegen
unschuldig erlittener Haft sowie sonstige Anweisungen in Strafsachen aus Kap. 76 Tit. 52

2) Zusammenstellung der Gnadentabellen.

1. Schön

2. Schön

3. Busch, MR.

1) Landesverrats- u. Spionagesachen (einschl. der Gnaden- 1. Krug sachen)

2) Nichtpol. Einzelstrafsachen aus

2.
a) Celle, Düsseldorf,
b) Frankfurt, Hamm, Jena,
c) Kassel, Köln, Stettin,
a) Albrecht
b, d) Pritsch
c) Wille

d) außerpreuß. Gebiete

3) Wichtigere Abgabesachen in außerpreuß. Angelegenheiten.

3a. Dr. Stolzenburg, LGR. (s. auch Ref. 1a)

Mitarbeit in nichtpol. Einzelstrafsachen aus Köln.

3. Wille

Rindermann

3b. Dr. Rempe, StAR.

Mitarbeit in nichtpol. Einzelstrafsachen aus
a) Frankfurt a.M.,
b) Hamm,
c) Jena u. den außerpreuß. Gebieten.
a,c) Ref. 2c
b) Ref. $2 d$

3c. Dr. Kelz, AGR. (vgl. auch Ref. 7a)

Abgabesachen in außerpreuß. Angelegenheiten.

Voigts

3d. Rindermann, $L R$.

Mitarbeit in nichtpol. Einzelstrafsachen aus
a) Celle
b) Düsseldorf.
a) Hoffmann
b) $\mathrm{Kelz}$

3e. Dr. Kramer, StAR. (s. auch Ref. 2d)

Mitarbeit in nichtpol. Einzelstrafsachen aus Kassel und

Hoffmann Stettin.

3f. Voigts, hamb. Richter (vgl. auch Ref. 7c)

Reisner

Vorbearb. für Ref. Busch zu Nr. 1. 
4. Dr. Kutzner, OLGR.

1) Internat. Einzelstrafsachen

2) Strafsachen aus dem Saargebiet u. Minderheitsstrafsachen

3) Pol. Einzelstrafsachen aus

1 u. 2. Mettgenberg

3.

a) Wille

b-e) Albrecht

d) Naumburg,

b) Jena,

e) den außerpreuß. Gebieten.

c) Kiel,

5. Wille, KGR.

1) Pol. Strafsachen aus

a) Berlin,

b) Stettin u. Kassel

2) a) Nichtpol.u.

b) pol. Strafsachen, in denen ein Angeklagter zum Tode verurteilt worden ist, von der Rechtskraft des Urteils bis zur Erledigung der Vollstreckung (unter Mitarb. des vor Eintritt der Rechtskraft zuständigen Sachbearbeiters).

6. Dr. Albrecht, LGDir.

1) Generalref. in pol. Strafsachen

1. Wille

2) Pol. Einzelstrafsachen aus

a) Breslau,

b) Düsseldorf, Frankfurt a. M., Hamm, Köln,

c) Königsberg u. Marienwerder.

2.

a,c) Wille

b) Kutzner
a) Albrecht
b) Kutzner
2.
a) Albrecht
b) Kutzner

6a. Toebert, AGR.

Mitarbeit in pol. Einzelstrafsachen aus

a) Berlin,

b) Jena, Königsberg, Marienwerder u. den außerpreuß. Gebieten.

a) Knapp

b) Meyer

6b. Dr. Knapp, LGR.

Mitarbeit in pol. Einzelstrafsachen aus
a) Breslau, Celle,
b) Kiel, Naumburg, Stettin.
a) Toebert,
b) Meyer

6c. Meyer, $L G R$.

1) Mitarbeit in pol. Einzelstrafsachen aus

1.
a) Düsseldorf, Köln,
b) Frankfurt a. M., Hamm, Kassel
2) Sämtliche Strafsachen gegen Geistliche.
a) Toebert
b) Knapp
2. Knapp

6d. Lauterbach, ARat (s. auch Abt. IV)

Salewski

Vorbearb. von internat. Einzelstrafsachen, von Auslieferungssachen aus dem Ausland nur die Einzelfälle im Verkehr mit Italien u. der Tschechoslowakei (Ref. Kutzner).

7. Dr. Krug, OStA (s. auch ZF)

1) Gnadensachen einschl. Niederschlagungen, soweit das Gnadenrecht durch den Führer u. Reichskanzler ausgeübt wird (ohne Landesverrats- u. Spionagesachen - s. Ref. 3)

2) Hochverratssachen, soweit sie in die Zuständigkeit des Volksgerichtshofs fallen

3) Einzelsachen betr. Strafregister des RJM.

1. Mettgenberg

2. Mettgenberg

3. Busch

7a. Dr. Kelz, AGR. (vgl. auch Ref. 3c)

Mitarbeit in Einzelsachen betr. Strafregister des RJM. 
7b. Dr. Reisner, GAss. (vgl. auch Ref. lb)

Voigts

Vorbearb. für Ref. Krug zu Nr. 1.

7c. Voigts, hamb. Richter (vgl. auch Ref. 3f)

Reisner

Vorbearb. für Ref. Krug zu Nr. 2.

8. Klee, RR. (s. auch Abt. VI)

Sauthoff

Transportwesen $u$. Transportkosten.

\section{Gruppe Strafvollzug}

Leiter: Ministerialdirektor Dr. Crohne

Zeichnung der Generalsachen, soweit sie nicht den Sachbearbeitern übertragen ist, u. der Einzelsachen von politischer, grundsätzlicher od. sonstiger besonderer Bedeutung - ausgenommen aus Ref. 9 die Sachen zu lc-e u. sonst. Besoldungssachen, die der Leiter der Abt. VI zeichnet.

Vertreter: Dr. Schmidt, MR., im einzelnen der Dienstälteste d. beteiligten MinRäte.

Arbeitsgebiete $\quad$ Vertreter

9. Dr. Schmidt, $M R$.

1) Allgemeine Angelegenheiten

1. Marx

a) Generalref. für Strafvollzug (Vollzug der Untersuchungshaft, der Freiheitsstrafen u. der mit Freiheitsentziehung verbundenen Maßregeln der Sicherung und Besserung), ohne Gesetzgebung (s. Abt. II)

b) Allgem. Beamtenangelegenheiten

c) Allgem. Finanzsachen, insbes. Haushaltssachen u. Regelung der Personalbewilligung

d) Rechnungssachen

e) Allgem. Besoldungssachen

f) Allgem. Verwaltungsangelegenheiten u. sonstige Angelegenheiten allgem. Art; $z u b-f$ : für den Bereich der Strafanstaltsverwaltung, jedoch ohne Angelegenheiten des Arbeitsbetriebes u. ohne Bausachen

g) Zulassung u. Ausbildung von Anwärtern für den Strafvollzugsdienst

2) Personal-, Verwaltungs- u. Baueinzelsachen aus

2.
a) Kassel,
b) Köln
3) Zeichnung in Einzelsachen, in denen
a) Hecker
b) Weddige
3. Marx

a) das Finanzreferat beteiligt,

b) eine Entscheidung über die Personalbemessung zu treffen ist.

10. Dr. Weddige, MR.

1) Allgemeine Angelegenheiten

a) Hygienische Einrichtungen der Gefangenenanstalten, Gesundheitswesen, ärztliche Versorgung der Gefangenen, Irrenabteilungen, Kranken- $u$. Tuberkuloseabteilungen (einschl. Bausachen)

b) Angelegenheiten der Hauswirtschaft, insbesondere Verpflegung, Bekleidung, Lagerung u. Beschaffungswesen
1.

a) Schmidt

b) Marx 


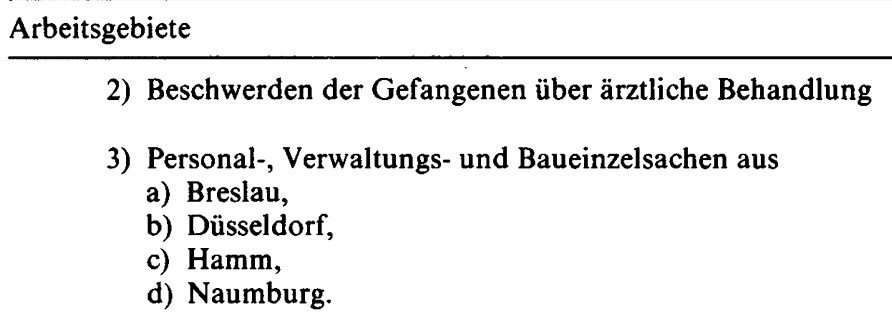

Vertreter
2. der zust. Bezirksref.
3.
a) Hecker
b) Schmidt
c) Marx
d) Hecker

11. Marx, $M R$.

1) Allgemeine Angelegenheiten
a) Landwirtschaftliche Betriebe
b) Arbeitswesen
c) Bausachen (einschl. Haftraumbeschaffung)
d) Besichtigungen durch Ausländer

2) Einzelsachen des Arbeitswesens

3) Personal-, Verwaltungs- u. Baueinzelsachen aus
a) Kiel,
b) Königsberg,
c) Marienwerder

1.
a) Schmidt
b u. c) Hecker
d) Schmidt
2. der zust. Bezirksref.
3.
a) Weddige
b) Hecker
c) Schmidt

4) Angelegenheiten der Strafgefangenen-Lager in Papenburg.

12. Hecker, JR.

1) Allgemeine Angelegenheiten

zugl. Korref.
a) Seelsorge
b) Bücherei
c) Unterricht
d) Gefangenen- u. Entlassenenfürsorge
e) Strafvollzugsstatistik

1.
a) Marx
b u. c) Schmidt
d) Marx
e) Schmidt

2) Sonderaufträge aus den Referaten $9 u .11$

3) Personal-, Verwaltungs- u. Baueinzelsachen aus

3.
a) Berlin,
b) Celle,
a) Marx
c) Frankfurt,
d) Stettin.
b) Schmidt
d u. e) Weddige

\section{Abteilung IV}

(Bürgerliches Recht, bürgerliche Rechtspflege)

Leiter: Ministerialdirektor Dr. Volkmar

Vertreter: Dr. Brandis, MR.

Arbeitsgebiete $\quad$ Vertreter

1. Dr. Brandis, $M R$.

1) Bürgerliches Recht:
a) Miet- u. Pachtrecht
b) Erbbaurecht
c) Familienrecht
(Vorbearb. Rexroth)
1 u. 3.
Maßfeller
2) Korref. für
2. -

a) Ref. la (Maßfeller) zu 1 bis 3

b) Heimstätten-, städt. Siedlungs-, Kleingarten- u. Städtebaugesetzgebung, Pachtschutzordnung (Ref.

Mitzschke).

3) Beteiligung beim Fürsorgerecht (Ref. 5 zu Nr. 1). 


\begin{tabular}{l} 
Arbeitsgebiete \\
\hline 1a. Maßfeller, $A G R$. \\
1) Personenstands- u. Standesamtssachen \\
2) Jugendwohlfahrt u. Fürsorgeerziehungssachen \\
3) Verhütung erbkranken Nachwuchses \\
4) Familienrechtl. Einzelsachen (s. auch Ref. 9b) \\
(zu 1-4: Korref. Brandis) \\
5) Schiedsmänner \\
6) Zivilrechtl. Einzelsachen aus Köln u. Naumburg (Korref. \\
Pätzold).
\end{tabular}

Vertreter

lb. Rexroth, StA.

Vorbearb. in:

1) Miet-, Pacht- u. Familienrecht (Ref. Brandis)

2) Zivilrechtl. Einzelsachen aus Hamm (Ref. Köhler).

2. Dr. Hesse, MR.

1) Grundbuchsachen

2) Angelegenheiten der freiw. Gerichtsbarkeit

3) Orts- u. Dorfgerichte

4) Korref. für Bürgerl. Gesetzbuch Allg. Teil (Ref. Mitzschke)

5) Korref. für zivilrechtl. Einzelsachen aus Celle, Stettin 5. (Ref. Mitzschke).

3. Dr. Pätzold, ORR. (s. auch ZS.)

1) Bürgerliches Recht

a) Schuldrecht (ohne Miete u. Pacht - s. Ref. 1) einschl. der Entschuldungsgesetzgebung (ohne ländliche Entschuldung - s. Abt. II)

b) Sachenrecht

c) Gesetzgebung auf dem Gebiete des Hypothekenbankwesens (Einzelsachen s. Abt. V) (ohne Schiffspfandbriefbanken u. öffentlichrechtliche Kreditanstalten s. Abt. V)

d) Mündelsicherheit, Devisenrecht

e) Aufwertung u. Nebengebiete.

2. Korref. für

a) Jagdrecht (Ref. Mitzschke)

b) Zivilrechtl. Einzelsachen (Ref. Maßfeller).

3a. Dr. Schultze, LGR.

1) Vorbearb. im Ref. Pätzold

a) Entschuldungsgesetzgebung

b) Gesetzgebung auf dem Gebiete des Hypothekenbankwesens

c) Mündelsicherheit, Devisenrecht

2) Sonderaufträge.

4. Dr. Mitzschke, LGR.

(s. auch ZF, ferner Berichterstattung im Staatsministerium

über $Z$ wangspensionierungsbeschwerden)

1) Bürgerl. Gesetzbuch Allg. Teil (Korref. Hesse)

1 u. 6. Hesse

2) Wasserrecht, Jagd-, Forst-, Fischereirecht (Jagdrecht:

Korref. Pätzold)

3) Heimstätten-, städtische Siedlungs-, Kleingarten- u. Städtebaugesetzgebung, Pachtschutzordnung (Korref. Brandis)

4) Bergrecht (ohne Recht der Gewerkschaften - s. Abt. V)

5) Schätzungssachen 
6) Zivilrechtl. Einzelsachen aus Celle u. Stettin (Korref. Hesse).

5. Dr. Koehler, MR. (s. auch LA.)

1) Soziale Gesetzgebung einschl. Unfall-, Kranken-, Alters-, Vogels Invaliden- und Angestelltenversicherung (bei Fürsorgerecht Beteiligung: Brandis)

2) Materielles Arbeitsrecht einschl. Recht der Hausangestellten

3) Zivilrechtl. Einzelsachen aus Breslau, Marienwerder u. Hamm (Vorbearb. für Hamm: Rexroth).

6. Dr. Vogels, MR. (s. auch Abt. II)

1) Bürgerliches Recht: Erbrecht

2) Konkursrecht, Vergleichsordnung, Anfechtung außerhalb des Konkurses

3) Vollstreckungsschutz für die Binnenschiffahrt

4) Zivilrechtl. Einzelsachen aus Düsseldorf u. Frankfurt a. M. (Vorbearb.: Pohle).

7. Kühnemann, LGR.

Pohle

1) Gewerblicher Rechtsschutz (Recht der Patente, Gebrauchsmuster, Geschmacksmuster, Warenzeichen, Schutz gegen unlauteren Wettbewerb)

2) Literarisches u. künstlerisches Urheberrecht, Verlagsrecht.

8. Dr. Jonas, $M R$.

1) Zivilprozeß u. Prozeßreform

1 u. 4. Maßfelder

2 u. 3. Jonas

Pon

Staud

Zwangsvollstreckungsverfahren (Beteiligung: Staud)

Völkerrechtl. Verträge auf dem Gebiete der bürgerl.

Rechtspflege

2) Anwaltsrecht

(zu 1 u. 2 Vorbearb.: Pohle)

3) Notariatswesen (Vorbearb.: Hornig)

4) Beteiligung an
a) Gerichtsverfassungsgesetz, Zivilprozeß (Erkenntnisver- fahren) (Ref. Staud)
b) Kostenwesen (Ref. Melsheimer)
c) Gerichtsvollzieherwesen (Ref. Lentz)
5) Zivilrechtl. Einzelsachen aus den nichtpreuß. Gebieten.

8a. Pohle, LGR.

Vorbearb.:

1) der Zivilprozeß- u. Anwaltssachen (Ref. Jonas)

2) der zivilrechtl. Einzelsachen aus Düsseldorf u. Frankfurt a. M. (Ref. Vogels).

9. Staud, $M R$.

1) Gerichtsverfassungsgesetz (Beteiligung: Jonas)

2) Zivilprozeß u. Prozeßreform: Erkenntnisverfahren einschl. des arbeitsgerichtlichen Verfahrens (Beteiligung Jonas) (zu 1 u. 2: Mitarbeit Fechner)

3) Mitwirkung bei grundsätzlichen Fragen der Justizstatistik (s. Abt. VI)

4) Zivilrechtl. Einzelsachen aus Kassel u. Kiel (Vorbearb.: Fechner)

5) Beteiligung an Zivilprozeß (Zwangsvollstreckung) (Ref. Jonas). 
9a. Dr. Fechner, LGR.

1) Mitarbeit in den Zivilprozeßsachen u. Gerichtsverfassungsfragen aus dem Ref. Staud

2) Vorbearb. der zivilrechtl. Einzelsachen aus Kassel u. Kiel.

9b. Lauterbach, ARat. (s. auch Abt. III)

1) Internat. Einzelsachen in Angelegenheiten des Zivilprozesses und der freiw. Gerichtsb. (Korref. Kriege s. Abt. V)

2) Vorbearb. für die Befreiung von der Beibringung des ausländ. Ehefähigkeitszeugnisses (s. Ref. Maßfeller).

10. Lentz, MR. (s. auch Abt. I, RJP u. LA)

Melsheimer

Gerichtsvollzieherwesen (Beteiligung: Jonas).

11. Dr. Dr. Wilke, OLGR. (s. auch StS. Dr. Schlegelberger)

Sonderaufträge, insbes. auf dem Gebiete des internat. Rechts.

12. Melsheimer, LGD.

1) Kosten- u. Stempelgesetzgebung (Beteiligung: Jonas s. auch Abt. VI)

2) Zivilrechtl. Einzelsachen aus Berlin u. Königsberg i. Pr. (zu 1 u. 2 Vorbearb.: Hornig).

12a. Hornig, $A G R$.

Vorbearb.:

1) Notariatswesen (Ref. Jonas)

2) Kostengesetz (Ref. Melsheimer)

3) der Einzelsachen aus Berlin u. Königsberg i. Pr. (Ref. Melsheimer).

\section{Jonas}

\section{Abteilung $V$}

(Handelsrecht, Verkehrsrecht, Völkerrecht und öffentliches Recht)

Leiter: Ministerialdirektor Richter

Vertreter: Quassowski, MR.

\section{Arbeitsgebiete}

1. Quassowski, $M R$.

1) Handelsrecht (ohne Handelsregister, Firmenrecht, handelsrechtlichen Arbeitsvertrag - s. Ref. 2 -, ohne Frachtrecht - s. Ref. 2, 3, 4) (Vorbearb.: Herbig)

2) Recht der G.m.b.H. u. der Genossenschaften einschl. der landesrechtl. u. der öffentl.-rechtlichen (ohne Genossenschaftsregister - s. Ref. 2). Bergrechtliche Gewerkschaften (Vorbearb. für Genossenschaften: Souchon, im übrigen Herbig)

3) Goldbilanzen. Kapitalherabsetzung in erleichterter Form. Bilanzerleichterungen. (Vorbearb.: Herbig)

4) Reform des Aktienrechts u. des Genossenschaftsrechts. Wirtschaftsprüfer. (Vorbearb. für Reform des Genossenschaftsrechts: Souchon, im übrigen Herbig)

5) Wechsel- u. Scheckrecht. (Vorbearb.: Souchon)

6) Bankrecht (ohne Hypothekenbanken - s. Abt. IV - u. Schiffspfandbriefbanken - s. Ref. 2). Bankdepotgesetz. Börsenrecht. (Vorbearb.: Souchon).
Vertreter

die mit der Vorbearb. betrauten Beamten 


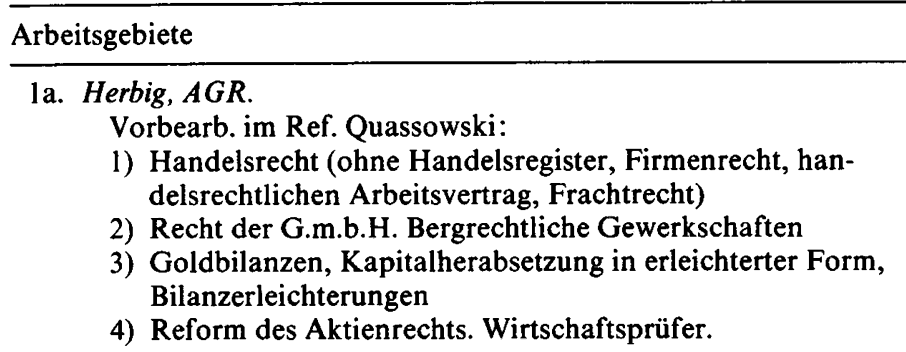

2. Krieger, MR. (s. auch LA)

1) Handelsregister und Genossenschaftsregister. Firmenrecht. s. zu 5. (Vorbearb.: Lenz) Handelsrechtlicher Arbeitsvertrag. (Vorbearb.: Souchon)

2) Schuldverschreibungsgesetz. (Vorbearb.: Souchon)

3) Öffentlichrechtl. Kreditanstalten. Schiffspfandbriefbanken. Hypothekenbankwesen ohne Gesetzgebung (diese s. Abt. IV)

4) Wirtschaftsrecht, insbes. Kartellrecht, Gewerberecht. (Vorbearb.: Lenz)

5) Seerecht

6) Privates u. öffentliches Versicherungsrecht (Sozialversicherung s. Abt. IV) (Vorbearb.: Lenz).

3. Quassowski

5. Koffka im übrigen die mit der Vorbearb. betrauten Beamten

2a. Dr. Lenz, LGD.

Vorbearb. im Ref. Krieger:

1) Handelsregister u. Genossenschaftsregister, Firmenrecht

2) Wirtschaftsrecht, insbes. Kartellrecht, Gewerberecht

3) Privates u. öffentliches Versicherungsrecht.

2b. Dr. Souchon, LGR. u. AGR.

Vorbearb. in

1) Handelsrechtl. Arbeitsvertrag (Ref. Krieger)

2) Recht der Genossenschaften (ohne Genossenschaftsregister), Reform des Genossenschaftsrechts (Ref. Quassowski)

3) Wechsel- u. Scheckrecht, Bankrecht (ohne Hypothekenbanken u. Schiffspfandbriefbanken), Bankdepotgesetz, Börsenrecht (Ref. Quassowski)

4) Schuldverschreibungsgesetz (Ref. Krieger).

3. Koffka, MR. (s. auch ZS)

Riese

1) Privates u. öffentliches Verkehrsrecht (Eisenbahnen, Kraftwagen, Wasserstraßen, Binnenschiffahrt, Internat. Flußkommissionen) ohne Seerecht - s. Ref. 2, ohne Luftrecht s. Ref. 4

2) Post- u. Telegraphenrecht, Funkrecht.

4. Dr. Riese, ORR.

1) Privates u. öffentliches Luftrecht

2) Militärrecht (ohne Militärstrafrecht - s. Abt. II-)

$1,2,4$.

3) Völkerrecht, soweit nicht in Ref. 6

Koffka

4) Sonderaufträge. 
Vertreter

5. Kritzinger, $M R$.

1) Staats- u. Verwaltungsrecht, Presserecht

Kriege

2) Beamtenrecht, Dienststrafrecht $u$. Dienststrafgerichte (zus. mit Abt. I Ref. Wittland u. Haastert)

3) Finanzrecht (soweit nicht in Abt. VI)

4) Angelegenheiten der ehemaligen Landes- und Standesherren (ohne Recht der Familiengüter, Hausvermögen usw. s. LA)

5) Kompetenzkonflikte

6) Enteignungsrecht

7) Gesetzgebung über Waffenbesitz und Waffenführung

8) Kirchenrecht.

5a. Kaulbach, LGR. (s. auch MB).

Mitarbeit im Ref. Kritzinger.

6. Dr. Kriege, $M R$.

1) Versailler Vertrag u. seine Auswirkungen, insbes. Ober-

Riese schlesien, Saargebiet

2) Recht der Minderheiten

3) Staatsangehörigkeitsfragen aus internat. Verträgen

4) Völkerbund, Internat. Schiedsgerichte

5) Konsularrecht, Exterritorialität

6) Legalisationen (Einzelsachen s. Witt)

7) Korref. für Ref. Lauterbach s. Abt. IV

8) Hinterlegungswesen, allg. u. Einzelsachen

9) Angelegenheiten aus dem ehemaligen Heroldsamt.

6a. Witt, ARat (s. auch LA).

Lauterbach

Legalisationen (Beglaubigungsvermerke).

\section{Abteilung VI}

(Preußische Justizverwaltungssachen)

Leiter: Ministerialdirigent Schneller

Leitung der Abteilung und Zeichnung der ihm vorbehaltenen Sachen aus Abt. I u. III (Gruppe Strafvollzug)

Vertreter: Dr. Reichau, MR; im einzelnen:

a) Hilfsarbeitersachen: der nach der preuß. Verfügung v. 17.4.34-ZB. $800-z u-$ ständige Beamte,

b) im übrigen der Dienstälteste der beteiligten Min.Räte u. soweit ein solcher nicht vorhanden: Reichau.

\section{Arbeitsgebiete}

Vertreter

1. Dr. Reichau, MR.

1) Besoldungssachen

2) Zeichnung der die Festsetzung des Anwärter- u. Besol-

1. Anders dungsdienstalters betreffenden Verfügungen

3) Allgemeine Angelegenheiten auf dem Gebiete des Ruhegehaltswesens u. der Hinterbliebenenfürsorge

4) Ersatzforderungen gegen den Fiskus

5) Durchführung des Luftschutzes - zu 1 u. 5: ohne Strafanstaltsverwaltung.

2. -

3. Osburg

4. Sandgänger

5. Herwig 
2. Herwig, $M R$.

1) Grundstücksverwaltung u. Bausachen (ohne die besonde-

1. Reinicke ren Strafanstalten s. Abt. III)

2) Zeichnung der Sachen aus Ref. 4.

2. -

3. Osburg, ORR. (s. auch Abt. I)

1) Einzelsachen auf dem Gebiete des Ruhegehaltswesens u. der Hinterbliebenenfürsorge

2) Reise- u. Umzugskosten

3) Beschäftigungstagegelder $u$. Wohnungsbeihilfen

4) Prämienfonds

5) Portosachen.

4. Dietz, ORR.

1) Bewilligungen aus den laufenden Mitteln für Bauunterhaltung

2) Änderung laufender Mietverträge, Bauunterhaltungsverträge usw.

3) Dienstwohnungen (ohne die der Oberlandesgerichtspräsidenten), Werk- u. Mietwohnungen.

5. Dr. Anders, $K G R$.

1) Bearbeitung folgender Sachen:

a) Allgemeine Finanzsachen, insbes. Haushaltssachen, Sparmaßnahmen $u$. Grundsätze für die Personalbemessung

b) Einzelsachen des Finanzreferats

c) Allgemeine Verwaltungssachen, für die kein besonderes Referat besteht

d) Gerichtsgemeinschaften

2) Mitwirkung bei

1, 4 u. 5. Gundlach

2 u. 3. Sauthoff

a) Angelegenheiten, die für die Einnahmen und Ausgaben der Justizverwaltung von grundsätzlicher Bedeutung sind

1. Reichau

b) Entscheidungen über die Verwendung (Ausschreibung, Übertragung usw.) von Planstellen

c) Einzelsachen, die die Besetzung der Behörden mit Hilfsarbeitern aller Art od. die Zubilligung von Sondervergütungen betreffen

- zu la, b u. 2b, c: ohne Strafanstaltsverwaltung.

6. Kollat, ORR.

Kosten- u. Stempelsachen, soweit sie nicht die Gesetzgebung (s. Abt. IV) oder ihre Durchführung (s. Ref. 11 Nr. 4) betreffen, ohne die Bezirke Berlin, Hamm u. Marienwerder (s.

Ref. 10).

7. Sauthoff, ORR.

1) Geschäftsübersichten

2) Justizstatistik (s. auch Abt. IV) ohne Kriminal- u. Strafvollzugsstatistik (s. Abt. II, III)

3) Terminkalender, Staatshandbuch u. ,Dienstlaufbahn“

4) Mitarbeit im Finanzref., insbes. Neufassung der Haushalts- 4. vorschriften

5) Geschäftsordnungen 1-3. Klee

Dietz, Klee, Sandgänger

a u. b)

unmittelbare

Vorlage beim Abt.-

Leiter

c) -

5 u. 6. Osburg 
Vertreter

6) Geschäftsgang

7) Zeichnung der Anweisungen über Reisekosten u. fortlaufende persönliche Bezüge der Beamten des PrJM., soweit es sich dabei lediglich um die formelle Ausführung der geltenden Bestimmungen handelt.

8. Dr. Reinicke, $L G R$.

Mitarbeit im Ref. 2.

9. Friedrich, $A G R$. (s. auch ZF u. RJP)

Anders

Angelegenheiten des Gemeinschaftslagers aus dem Geschäfts-

7. Urlacher bereich der Abt. VI.

10. Dr. Sandgänger, $A G R$.

(außerdem Berichterstattung im Staatsministerium über

Zwangspensionierungsbeschwerden)

1) Mitarbeit im Ref. 1 Nr. 4

2) Kosten- u. Stempelsachen, soweit sie nicht die Gesetzgebung (s. Abt. IV) oder ihre Durchführung (s. Ref. 11) betreffen, aus Berlin, Hamm u. Marienwerder

3) Sonderaufträge.

11. Klee, RR. (s. auch Abt. III)

1) Rechnungslegung u. Rechnungsprüfung 1. Anders

2) Kassenwesen (allgemeine u. Einzelsachen) 2. Sauthoff

3) Kassendefekte

4) Durchführung der Kosten- u. Steuergesetze.

1.-

2. Kollat

3 , 4. Kollat

1. Anders

2. Osburg

\section{Abteilung RJP}

(Ausbildung)

Leiter: Präsident Dr. Palandt

Vertreter: Vizepräsident Creutzfeldt; soweit die Vertretung in den Ministerialgeschäften nicht dem Vizepräsidenten besonders übertragen ist (s. Ref. 1), vertreten im einzelnen die jeweiligen Sachbearbeiter.

Vertreter

1. Creutzfeldt, V.-Präs.

1) Zeichnung aller Sachen, die die Ausbildung der Beamten des oberen, mittleren u. unteren Justizdienstes, der Amtsanwälte u. Gerichtsvollzieher betreffen

2) Justizoffiziantenwitwenkasse, Lautheriusstiftung, Mühl- Palandt bachstiftung, Kriegsgedächtnis- u. Kriegspatenstiftung.

2. Lentz, MR. (s. auch Abt. I, IV u. LA.)

1) Ausbildung der Gerichtsvollzieher u. Gerichtskassen-

Osburg vollzieher

2) Ausbildung der Amtsanwälte. 
3. Willers, MR. (s. auch Abt. I)

Lentz

Ausbildung der Beamten des oberen, mittleren u. unteren

Dienstes.

4. Dr. Richter, KGR. (s. auch Ref. 8)

1) Angelegenheiten des Reichs-Justizprüfungsamts (einschließlich seiner Besetzung - diese unter Mitwirkung von Abt. I)

2) Ausbildung der Richter, Staatsanwälte u. Rechtsanwälte, soweit nicht auf AGR. Last u. AGR. Friedrich übertragen

3) Personalien der Referendare.

Zu 2-4: einschl. der Maßnahmen zur Fortbildung der festangestellten Beamten.

5. Kühn, ORR.

Unterhaltszuschüsse, Prüfungsgebühren, Reisekosten zur großen Staatsprüfung.

6. Last, $A G R$. (s. auch Ref. 9)

1) Ernennung zum Gerichtsassessor, Festsetzung des Dienstalters

2) Mitarbeit im Ref. Richter.

7. Friedrich, $A G R$. (s. auch Ref. 10, Abt. ZF. u. VI)

1) Gemeinschaftslager (Einzelsachen)

2) Mitarbeit im Ref. Richter.

Last u. Friedrich

Reichs-Justizprüfungsamt

Verteilung der Verwaltungsgeschäfte

Präsident: Dr. Palandt

Creutzfeldt

8. Dr. Richter, KGR. (s. auch Ref. 4)

Last

Aufsicht über die Prüfungsämter (einschl. Personalien).

9. Last, $A G R$. (s. auch Ref. 6)

1) Zulassung zur großen Staatsprüfung

2) Genehmigung einer Wiederholung der Prüfung

3) Auswahl der geschichtlichen Aufgaben für die 1. u. 2. Prüfung.

10. Friedrich, AGR. (s. auch Ref. 7, Abt. ZF u. VI)

Jacob

Friedrich

Last

Friedrich

Mitarbeit bei der Auswahl der juristischen Aufgaben für die

1. Prüfung.

\section{Abteilung LA}

(Familiengüter u. Hausvermögen)

\begin{tabular}{l} 
Arbeitsgebiete \\
\hline 1. Leiter: MDirigent Prof. Dr. Klässel, Präsident des Landesamts \\
für Familiengüter. \\
1) Zeichnung in Angelegenheiten betr. die Aufsicht über die \\
Geschäftsführung der Auflösungsämter für Familiengüter
\end{tabular}

Vertreter

Palandt

Zeichnung in Angelegenheiten betr. die Aufsicht über die 
2) Zeichnung in Thronlehnsangelegenheiten, soweit dabei das Pr.JM. in Betracht kommt

3) Zeichnung in den die Familiengüter u. Hausvermögen betreffenden Angelegenheiten zu 2 und 3 sind ausgenommen die Fälle, in denen die Genehmigung zu einem Beschluß des Landesamts in Frage kommt.

2. Dr. Koehler, MR. (s. auch Abt. IV)

Hesse

1) Angelegenheiten der Auflösungsämter

2) Familiengüter u. Hausvermögen, Familienstiftungen, hohenzoll. Personenstandsregister, Ausführung des Adelsgesetzes.

3. Witt, ARat (s. auch Abt. V)

Machule, MA.

1) Fideikommißstatistik

2) Mitarbeit bei der Beaufsichtigung der Kostenerhebung der Auflösungsämter

3) Bestimmung des für Waldgüter u. Schutzforsten zuständigen Grundbuchamts (ohne Zeichnung).

\section{Landesamt für Familiengüter}

Präsident: Ministerialdirigent Prof. Dr. Klässel

Richterliche Mitglieder:

Lentz, MR. (s. auch Abt. I, IV, RJO)

Krieger, MR. (s. auch Abt. V)

Hesse

Dr. Koehler, MR. (s. oben u. Abt. IV).

Reichau

Anders

Dr. Bohnen,

ErbhofGR 


\title{
Anlage 2
}

\section{Reichsjustizministerium \\ Geschäftsverteilungsplan \\ Stand vom Frühjahr 1941}

Akten des Reichsjustizministeriums

Bundesarchiv, Sign. R 22/56, 57, 58

[Stand vom April 1941]

\author{
Minister: \\ Mit der Führung der Geschäfte beauftragt: \\ Staatssekretär Dr. Dr. Schlegelberger. *) \\ Zur persönlichen Verfügung: \\ Dr. Gramm, ORegRat \\ Ebersberg, LGRat \\ Staatssekretär Dr. Freisler \\ Zur persönlichen Verfügung: \\ Dr. von Hackwitz, ORegRat \\ Stier, StA. \\ Ebert, MinRat (für bes. Aufträge) Einberufen \\ Joppich, LGDir. Abgeordnet nach dem Haag \\ Mitarbeiter: \\ Dr. Geißeler, LGRat Einberufen \\ Dr. Nassauer, StA. Einberufen \\ Geschäftsbereich
}

A. 1. Abt. I Allgemeines und Personalsachen nach der Verteilung unter B

2. Abt. II

3. Abt. III mit Unterabt. IIIA, B, C, D

4. Abt.V mit Ausnahme des Handels-, Verkehrs- und Wirtschaftsrechts sowie des Pri-

5. Abt. VI senrechts

6. a) Abt. VII

b) Gemeinschaftslager

7. Übernahme der Assessoren

8. Betreuung der Umsiedler

9. Deutsche Justiz, Presse und Rundfunk, Bücherei

*) StS. Dr. Schlegelberger hat sich die das Reichsgericht und die Reichsanwaltschaft beim Reichsgericht, die die neuen Gebiete (Elsaß, Lothringen, Luxemburg, Eupen, Malmedy, Danzig und die eingegliederten Ostgebiete) betreffenden Angelegenheiten sowie das Prisenrecht vorbehalten. 
B. 1. Personalsachen und andere Angelegenheiten des Ministeriums

2. Volksgerichtshof

Reichsanwaltschaft beim Volksgerichtshof

Reichsjustizprüfungsamt

3. Gerichte und Staatsanwaltschaften in den Oberlandesgerichtsbezirken:

Berlin

Braunschweig

Breslau

Celle

Dresden

Hamburg

Jena

Kassel

Kattowitz

Kiel

Leitmeritz

Oldenburg

Posen

Prag

Rostock

Stettin

sowie die Strafvollzugsbehörden aller Bezirke

4. Generalgouvernement

\section{Unterstaatssekretär Dr. Hueber}

Zur persönlichen Verfügung:

Haupt, KGRat

Dr. Schlenz, LGRat

Geschäftsbereich

A. 1. Abt. I Personalsachen nach der Verteilung unter B

2. a) Abt. IV Bürgerliches Recht und bäuerliches Recht

b) Oberstes Fideikommißgericht

3. Aus Abt. V: Handels-, Verkehrs- und Wirtschaftsrecht

B. 1. Reichspatentamt

2. Reichserbhofgericht

3. Gerichte und Staatsanwaltschaften in den Oberlandesgerichtsbezirken:

Bamberg

Darmstadt

Düsseldorf

Frankfurt

Graz

Hamm

Innsbruck

Karlsruhe

Köln

Königsberg (ohne die eingegl. Ostgebiete)

Linz

Marienwerder

München

Naumburg

Nürnberg

Stuttgart

Wien

Zweibrücken 
4. Rechtsanwälte und Notare

5. Patentanwälte

Aufträge von besonderer politischer Bedeutung

Sommer, MinRat

Deutsche Justiz

Dr. Krug, MinRat

Mitarb. Dr. Ehrhardt, StA.

Vertreter Dr. Ehrhardt, StA.

Geschäftl. Verwaltung:

Lutterloh, MinDirig.

Vertreter Dr. Grussendorf, MinRat

Presse und Rundfunk

Dr. Krug, MinRat

Mitarb. Dr. Voß, EStA.

Woitschach, KGR. $\}$ Einbe-

Vertreter Dr. Ehrhardt, StA.

Dr. Ehrhardt, StA.

Fritsch, LGR.

Dr. Schneyder, RegRätin

Joppich, LGDir. Abgeordnet

Bücherei

Last, KGRat

Vertreter Manske, AGRat

Ministerialbürodirektor

Stadermann, ORegRat

Vertreter Andresen, ARat

Betriebsluftschutzleiter

Andresen, ARat

Vertreter Bohl, ARat

[Stand vom April 194I]

Abteilung I

(Personal- und Organisationssachen)

Leiter: $\quad$ Ministerialdirektor Dr. Nadler

Vertreter: Ministerialdirigent Lutterloh, innerhalb ihrer Arbeitsgebiete die Ministerialdirigenten Dr. Sauer und Willers.

Der Abteilungsleiter zeichnet auch die ihm vorbehaltenen Sachen aus III D.

Die Einzelsachen, die die Besetzung der Behörden mit Hilfskräften aller Art, das Besoldungswesen oder die Zubilligung von Sondervergütungen betreffen, zeichnet der Leiter der Abteilung VI.

\begin{tabular}{|c|c|}
\hline Arbeitsgebiete & Vertreter \\
\hline $\begin{array}{l}\text { 1. Lutterloh, Ministerialdirigent } \\
\text { 1) Zeichnung der Verfügungen in den Angelegenheiten der } \\
\text { Rechtsanwälte und Notare (Ref. } 13 \text { - Schoetensack } \\
\text { - Nr.1 bis 5; außerdem bezüglich der Notare aus Ref. } 20 \\
\text { - Miethsam -) }\end{array}$ & $\begin{array}{l}\text { Zu } 1 \text { und } 2 . \\
\text { Willers; Lentz für } \\
\text { die Angelegenhei- } \\
\text { ten aus dem Ref. } 5 \text {. }\end{array}$ \\
\hline
\end{tabular}


2) Zeichnung der Verfügungen in Angelegenheiten der Beamten des höheren Justizdienstes (mit Ausnahme der Beamten mit Einzelgehältern) betreffend
a) die Entlassung auf Antrag und die Zurruhesetzung
b) die Nebenbeschäftigung
c) die Beurlaubung
d) die Bewilligung von Beihilfen und Unterstützungen

3) Zeichnung der Verfügungen in Sachen der Verleihung der Treudienstehrenzeichen, soweit sie sich nicht der Abt.Leiter vorbehalten hat

4) Personalsachen der Richter und Verwaltungssachen aus
a) Köln
b) Oldenburg

5) Dolmetscher

Willers

Dr. Miethsam

Dr. Grussendorf

Willers

2. Dr. Sauer, Ministerialdirigent (s. auch Abt. VI)

Personalsachen der Beamten aller Dienstzweige und Verwaltungssachen des Reichspatentamts

Mitarbeiter: Dr. Dr. Schade, Kammergerichtsrat

3. Haastert, Ministerialdirigent. Einberufen

Kriegsvertreter

1) Allgemeine Beamtenangelegenheiten

Ruppert (Nr.9)

2) Allgemeine Angelegenheiten der Beamten des höheren Ju-

Ruppert (Nr.9) stizdienstes

Mitarbeiter: Dr. Dörken, Landgerichtsrat. Einberufen

3) Personalsachen der im Ministerium beschäftigten Beamten des höheren Dienstes

Kriegsvertreter

Sommer (Nr. 16)

4) Personalsachen der Richter und Verwaltungssachen aus

Dr. Koehler (Nr. 8)

Düsseldorf

5) Betreuung der Jungjuristen

Dr. Grussendorf

(Nr. 12)

4. Willers, Ministerialdirigent (s. auch Abt. VII)

1) Zeichnung der Verfügungen in den Angelegenheiten

a) der Beamten des gehobenen, mittleren und einfachen Justizdienstes

b) der Angestellten und Lohnempfänger, soweit sie sich der Abt.Leiter nicht vorbehalten oder den Referenten übertragen hat

2) Allgemeine Angelegenheiten des gehobenen, mittleren und Gundlach einfachen Justizdienstes

Mitarbeiter: Gundlach, Regierungsrat

3) Personalsachen der Richter und Verwaltungssachen aus
a) Hamburg
b) Hamm
Koehler
Zehrfeld

Mitarbeiter: Dr. Uppenkamp, Sen.Präsident

4) Einzelsachen der Beamten des gehobenen, mittleren und

Lentz einfachen Justizdienstes aus

Hamburg

Mitarbeiter: Dr. Uppenkamp, Sen.Präsident

5) Korreferat für Ref. 21 (Gundlach) zu 1 und 2 
5. Lentz, Ministerialrat (s. auch Abt. IV, VII)

1) Allgemeine Angelegenheiten der Gerichtsvollzieher und Justizvollstreckungsassistenten, soweit nicht Abt. IV zuständig ist

2) Einzelsachen der Gerichtsvollzieher und der Justizvollstreckungsassistenten

3) Unterstützungen und Beihilfen

4) Allgemeine und Einzelsachen der Beamten der Amtsanwaltslaufbahn

5) Einzelsachen der Beamten des gehobenen, mittleren und einfachen Justizdienstes ausRostock

Ruppert

Osburg

Zehrfeld

Willers

Osburg

6. Zehrfeld, Ministerialrat (s. auch Abt. VI)

1) Personalsachen der Richter und Verwaltungssachen aus
a) Dresden (als Kriegsvertreter für Ref. 10 - Bälz - zu 2a)
b) Naumburg
a) Koehler
b) Schade

2) Einzelsachen der Beamten des gehobenen, mittleren und einfachen Justizdienstes aus
a) Braunschweig
b) Darmstadt
Lentz
c) Oldenburg
Gundlach
Gundlach
d) Dresden
Lentz

Mitarbeiter: Kurze, Kammergerichtsrat. Einberufen

7. Dr. Dörffler, Ministerialrat (s. auch Abt. II)

1) Personalsachen der Beamten aller Dienstzweige und Verwaltungssachen
a) der Reichsanwaltschaft beim Reichsgericht
b) der Reichsanwaltschaft beim Volksgerichtshof
c) des Reichsgerichts
d) des Volksgerichtshofs
e) des Reicnserbhofgerichts
zu c-e als Kriegsvertreter für Ref. 10 (Bälz) zu 1

Dr. Wittland
Dr. Wittland
Dr. Sauer
Dr. Wittland
Dr. Wittland

2) Personalsachen der Staatsanwälte und Verwaltungssachen

Dr. Wittland der Staatsanwaltschaften einschließlich des Generalgouvernements

Beteiligung bei den Bezirken: Bamberg, Graz, Innsbruck, Karlsruhe, Linz, München, Nürnberg, Stuttgart, Wien und Zweibrücken

3) Abordnungen und Versetzungen von Beamten des höheren Justizdienstes in den Bereich anderer Verwaltungen oder von Parteidienststellen

4) Anträge auf Bestellung von Beamten zu Mitgliedern von Berufsgerichten und der Schiedsgerichte des Reichsnährstandes

Zu 3 und 4: unter Beteiligung der Bezirksreferenten.

8. Dr. Koehler, Ministerialrat (s. auch Abt. IV und Ob. Fideikommiß-Gericht)

Personalsachen der Richter und Verwaltungssachen aus
a) Frankfurt
b) Jena
c) Düsseldorf (als Kriegsvertreter für Ref. 3 (Haastert) zu 4)
d) Rostock (als Kriegsvertreter für Ref. 15 (Friedrich) zu 1b)
e) Stettin (als Kriegsvertreter für Ref. 10 (Bälz) zu 2b)

Dr. Wilhelmi

Zehrfeld

Dr. Wilhelmi

Dr. Miethsam

Dr. Schade 
9. Ruppert, Ministerialrat (s. auch Abt. V, VI)

1) Allgemeine Beamtenangelegenheiten (als Kriegsvertreter für Ref. 3 (Haastert) zu 1)

2) Allgemeine Angelegenheiten der Beamten des höheren Ju-

Dr. Sauer

Dr. Sauer stizdienstes (als Kriegsvertreter für Ref. 3 (Haastert) zu 2) Mitarbeiter $z u 1$ und 2: Reineck, Landgerichtsrat

3) Personalsachen der Beamten aller Dienstzweige (ausgenommen Gerichtsvollzieher, Justizvollstreckungsassistenten und Amtsanwälte) und der Notare und Verwaltungssachen aus
a) Karlsruhe
Dr. Miethsam
b) Stuttgart
Dr. Miethsam

zu b): als Kriegsvertreter für Ref. 10 (Bälz) zu 3)

10. Bälz, Ministerialrat (s. auch Ob. Fideikommißgericht)

Zur Militärverwaltung nach Paris abgeordnet

1) Personalsachen der Beamten aller Dienstzweige und Verwaltungssachen
a) des Reichsgerichts
b) des Volksgerichtshofs
c) des Reichserbhofgerichts

2) Personalsachen der Richter und Verwaltungssachen aus
a) Dresden
b) Stettin
Mitarbeiter: Kurze, Kammergerichtsrat. Einberufen

3) Personalsachen der Beamten aller Dienstzweige (ausgenommen Gerichtsvollzieher, Justizvollstreckungsassistenten und Amtsanwälte) und der Notare und Verwaltungssachen aus Stuttgart

\section{Dr. Wittland, Ministerialrat}

1) Organisationssachen grundsätzlicher Art, die über den Be- Willers zirk eines Oberlandesgerichts hinausgehen

2) Mitwirkung bei den Organisationssachen in den Bezirken

3) Allgemeine Angelegenheiten des Dienststrafrechts und der Dienststrafgerichtsbarkeit, Beteiligung am Dienststrafrecht (vgl. Abt. V)

4) Angelegenheiten betr. Anwendung der Sozialversicherungsgesetze auf Beamte, Angestellte und Lohnempfänger

5) Personalien der Richter und Verwaltungssachen aus
a) Celle
b) Kassel
c) Kiel
d) Darmstadt
e) des Landeserbhofgerichts

6) Personalsachen der Beamten aller Dienstzweige und Verwaltungssachen aus
a) Leitmeritz
b) Posen
c) Prag
d) dem Generalgouvernement

Kriegsvertreter:

Dr. Dörffler

(Nr.7)

\author{
Zehrfeld (Nr.6)
}

Dr. Koehler (Nr. 8)

Ruppert (Nr.9)
Willers
Dr. Dörffler

Zehrfeld

Beier

Dr. Grussendorf

Dr. Grussendorf

Dr. Dörffler

Dr. Dörffler

Dr. Dörffler 
mit Ausnahme der Personalsachen der Staatsanwälte, der Gerichtsvollzieher, Justizvollstreckungsassistenten, Amtsanwälte und der Verwaltungssachen der Staatsanwaltschaften.

Mitarbeiter: Dr. Wilhelmi, Oberlandesgerichtsrat

12. Dr. Grussendorf, Ministerialrat

1) Personalsachen der Richter und Verwaltungssachen aus

a) Berlin

b) Königsberg

c) Marienwerder

d) Braunschweig

2) Personalsachen der Beamten aller Dienstzweige und Verwaltungssachen aus

Danzig

mit Ausnahme der Personalsachen der Staatsanwälte, der Gerichtsvollzieher, Justizvollstreckungsassistenten, Amtsanwälte und der Verwaltungssachen der Staatsanwaltschaften

3) Gestaltung des Anwärterdienstes für den höheren Justizdienst

4) Übernahme von Assessoren als Anwärter

5) Austausch von in der Entschuldung tätigen Assessoren

6) Einsatz und Verteilung der Kräfte aller Laufbahnen im gesamten Bereich der Reichsjustizverwaltung aus Anlaß der Einberufungen zur Wehrmacht, der Wiedereinstellung von Ruhestandsbeamten und dergl.

7) Betreuung der Jungjuristen (als Kriegsvertreter für Ref. 3 Haastert - zu 5)

Mitarbeiter: Frohwein, Landgerichtsrat

13. Schoetensack, Ministerialrat

1) Allgemeine Angelegenheiten und Einzelsachen der Rechts- Dr. Wittland anwälte

2) a) Allgemeine Angelegenheiten der Notare

Dr. Wittland

b) Einzelsachen der Notare mit Ausnahme der Bezirke: Bamberg, Karlsruhe, München, Nürnberg, Stuttgart, Zweibrücken

3) Beteiligung an der Gesetzgebung in Angelegenheiten der Rechtsanwälte und Notare

4) Übernahme von Assessoren

Dr. Miethsam

Dr. Miethsam

Dr. Dörffler

Dr. Dörffler

Dr. Schade

Ruppert

Ruppert

Beier

Dr. Miethsam

Dr. Wittland

Dr. Wittland

Dr. Wittland

a) in den anwaltlichen Probedienst und als Anwärter

b) in den Probe- und Anwärterdienst für das Amt des Notars mit Ausnahme der Bezirke: Bamberg, Karlsruhe, München, Nürnberg, Stuttgart und Zweibrücken

5) Gestaltung des anwaltlichen Probe- und Anwärterdienstes und des Probe- und Anwärterdienstes für das Amt des Notars

Mitarbeiter: Dr. Schwarz, Landgerichtsrat

Dr. Walenta, Landgerichtsdirektor, für die Bezirke Graz, Innsbruck, Linz und Wien Dr. Dr. Schade, Kammergerichtsrat, für die Bezirke Danzig, Posen, Kattowitz, den Landgerichtsbezirk Zichenau (Oberlandesgerichtsbezirk Königsberg) und das Generalgouvernement

Dr. Wittland 
Vertreter

14. Beier, Ministerialrat

1) Personalsachen der Beamten aller Dienstzweige (ausgenommen Gerichtsvollzieher, Justizvollstreckungsassistenten und Amtsanwälte) und Verwaltungssachen aus
a) Linz
b) Wien
Dr. Walenta
Dr. Walenta

2) Korreferat für Ref. 25 (Dr. Walenta) zu 1

15. Friedrich, Kurt, Ministerialrat (s. auch Abt. VI, VII). Einberufen

1) Personalsachen der Richter und Verwaltungssachen aus
a) Breslau
b) Kattowitz
c) Rostock

2) Jungbeamtenaustausch

Kriegsvertreter

Dr. Miethsam

(Nr.20)

Dr. Miethsam

(Nr. 20)

Dr. Koehler (Nr. 8)

16. Sommer, Ministerialrat (s. auch Abt. VI)

1) Personalsachen der im Ministerium beschäftigten Beamten

Dr. Grussendorf des höheren Dienstes (als Kriegsvertreter für Ref. 3

- Haastert - zu 3)

Mitarbeiter: Hawranke, Regierungsrat

2) Korreferat für Ref. 19 (Stadermann)

17. Osburg, Oberregierungsrat (s. auch Abt. VI)

Einzelsachen der Beamten des gehobenen, mittleren und einfachen Justizdienstes aus
a) Breslau
b) Hamm
c) Kattowitz
d) Naumburg
Bender
Bender
Bender
Bender

18. Bender, Oberregierungsrat (s. auch Abt. VI)

Einzelsachen der Beamten des gehobenen, mittleren und einfachen Justizdienstes aus.
a) Celle
b) Jena
Osburg
Osburg
c) Kiel
d) Königsberg
e) Marienwerder
f) Stettin
Osburg
Gundlach
Gundlach
Osburg

19. Stadermann, Oberregierungsrat, Ministerialbürodirektor (s. auch Abt. VI)

Personalsachen der im Ministerium beschäftigten
a) Beamten des gehobenen, mittleren und einfachen Dienstes
b) Angestellten
c) Lohnempfänger
Korreferent: Sommer, Ministerialrat
Mitarbeiter: zu a) Otte, Regierungsrat
zu b) und c) Hawranke, Regierungsrat

20. Dr. Miethsam, Oberregierungsrat
1) Personalsachen der Richter und Verwaltungssachen aus Breslau und Kattowitz (als Kriegsvertreter für Ref. 15 (Friedrich) zu la) u. b)
Dr. Wilhelmi 
2) Personalsachen der Beamten aller Dienstzweige (ausgenommen Gerichtsvollzieher, Justizvollstreckungsassistenten und Amtsanwälte) und der Notare und Verwaltungssachen aus
a) Bamberg
b) München
c) Nürnberg
d) Zweibrücken

Dr. Wilhelmi

21. Gundlach, Regierungsrat

1) Allgemeine und Einzelsachen der Versorgungsanwärter Willers

2) Allgemeine und Einzelsachen der Angestellten und Lohn- Willers empfänger Korreferent zu 1 u. 2 MinDirig. Willers

3) Mitarbeit im Ref. 4 (Willers) zu 2

4) Einzelsachen der Beamten des gehobenen, mittleren und einfachen Justizdienstes aus Köln

5) Abordnungen und Versetzungen von Beamten des gehobenen, mittleren und einfachen Justizdienstes sowie von Angestellten und Arbeitern in den Bereich anderer Verwaltungen oder von Parteidienststellen

22. Otte, Regierungsrat

1) Angelegenheiten der Ruhestandsbeamten des Ministe- Hawranke riums (ohne höheren Dienst)

2) Mitarbeit im Ref. 19 (Stadermann) zu a)

23. Hawranke, Regierungsrat

1) Einzelsachen der Beamten des gehobenen, mittleren und einfachen Justizdienstes aus

Düsseldorf

2) Mitarbeit im Ref. 16 (Sommer) zu 1 und im Ref. 19 (Stadermann) zu b) und c)

24. Dr. Uppenkamp, Sen.Präs. (s. auch Abt. V, VI)

Mitarbeit im Ref. 4 (Willers) zu 3 und 4

25. Dr. Walenta, Landgerichtsdirektor

1) Personalsachen der Beamten aller Dienstzweige (ausge-

Bender

Willers nommen Gerichtsvollzieher, Justizvollstreckungsassistenten und Amtsanwälte) und Verwaltungssachen aus

a) Graz

b) Innsbruck

Korreferent: Beier, Ministerialrat

2) Mitarbeit im Ref. 13 (Schoetensack) für die Bezirke Graz, Innsbruck, Linz und Wien

26. Dr. Wilhelmi, Oberlandesgerichtsrat

1) Einzelsachen der Beamten des gehobenen, mittleren und einfachen Justizdienstes aus
a) Frankfurt

b) Kassel

2) Mitarbeit im Ref. 11 (Wittland)

27. Kurze, Kammergerichtsrat. Einberufen

Mitarbeit im Ref. 6 (Zehrfeld) und 10 (Bälz) 
Vertreter

28. Dr. Dr. Schade, Kammergerichtsrat

Willers

1) Einzelsachen der Beamten des gehobenen, mittleren und einfachen Justizdienstes aus dem Kammergerichtsbezirk

2) Mitarbeit im Ref.2 (Dr. Sauer) und im Ref. 13 (Schoetensack) für Danzig, Posen, Kattowitz, den LGBezirk Zichenau (OLGBezirk Königsberg) u. das Generalgouvernement

29. Frohwein, Landgerichtsrat

Mitarbeit im Ref. 12 (Dr. Grussendorf)

30. Dr. Schwarz, Landgerichtsrat

Mitarbeit im Ref. 13 (Schoetensack)

31. Dr. Dörken, Landgerichtsrat. Einberufen

Kriegsvertreter

Mitarbeit im Ref. 3 zu 1 und 2

Reineck

32. Reineck, Landgerichtsrat

Mitarbeit im Ref.9 (Ruppert)

[Stand vom März 1941]

\section{Abteilung II}

Strafgesetzgebung (Strafrecht, Strafprozeß, Strafvollzug, Auslieferungsrecht, zwischenstaatliche Rechtshilfe in Strafsachen), bäuerliches Recht

Leiter: Ministerialdirektor Ernst Schäfer

Vertreter: Ministerialdirigenten GRR. Dr. Leopold Schäfer, Dr. Suchomel, Dr. Mettgenberg, Dr. Vogels

je für ihr Arbeitsgebiet, für die übrigen Sachen der jeweils anwesende älteste Ministerialdirigent (oder Ministerialrat) vorbehaltlich einer Sonderbestimmung des Abteilungsleiters im Einzelfall.

\begin{tabular}{l} 
Arbeitsgebiete \\
\hline Dr. Leopold Schäfer, GRR. Ministerialdirigent \\
Zeichnung der Verfügungen in folgenden Sachen: \\
1) Strafregisterwesen, \\
2) Militärstrafrecht und Militärstrafgerichtsordnung sowie \\
verwandte Gesetze, \\
3) Gebühren- und Kostengesetze.
\end{tabular}

(Bei Fragen von grundsätzlicher Bedeutung oder besonderer Wichtigkeit, insbesondere stets, wenn der Herr Minister oder der Herr Staatssekretär beteiligt ist, Vorbehalt der Zeichnung durch den Abteilungsleiter.)

2. Dr. Suchomel, Ministerialdirigent

Zeichnung der Verfügungen in folgenden Sachen:

1) Rechtsangleichung in der Ostmark,

2) Rechtsangleichung in den sudetendeutschen Gebieten,

3) Österreichisches und sudetendeutsches Landesstrafrecht,

4) Rechtsfragen des Protektorats Böhmen und Mähren.

(Bei Fragen von grundsätzlicher Bedeutung oder besonderer Wichtigkeit, insbesondere stets, wenn der Herr Minister oder der Herr Staatssekretär beteiligt ist, Vorbehalt der Zeichnung durch den Abteilungsleiter.)

\section{Vertreter}

Abteilungsleiter

Abteilungsleiter 
3. Dr. Mettgenberg, Ministerialdirigent

Zeichnung der Verfügungen in folgenden Sachen:

Zwischenstaatliches Strafrecht (einschl. Verfahren und Vollzug), Auslieferungsrecht und sonstige zwischenstaatliche Rechtshilfe in Strafsachen (soweit Maßnahmen im Wege der Gesetzgebung oder zwischenstaatliche Abmachungen, die eine über den Einzelfall hinausgehende Regelung treffen, in Frage kommen).

(Bei Fragen von grundsätzlicher Bedeutung oder besonderer Wichtigkeit, insbesondere stets, wenn der Herr Minister oder der Herr Staatssekretär beteiligt ist, Vorbehalt der Zeichnung durch den Abteilungsleiter.)

4. Dr. Vogels, Ministerialdirigent

Zeichnung der Verfügungen in folgenden Sachen:

Abteilungsleiter

1) Erbhofrecht,

2) Ländliche Entschuldung,

3) Ländliche Siedlung und Rentengutsgesetzgebung.

(Bei Fragen von grundsätzlicher Bedeutung oder besonderer Wichtigkeit, insbesondere stets, wenn der Herr Minister oder der Herr Staatssekretär beteiligt ist, Vorbehalt der Zeichnung durch den Abteilungsleiter.)

5. Rietzsch, Ministerialrat

1) Strafgesetzbuch,

2) Strafrechtliche Nebengesetze des Reichs, soweit nicht besonders zugeteilt,

3) Landesstrafrecht (mit Ausnahme des österreichischen, sudetendeutschen und des in den eingegliederten Ostgebieten geltenden Landesrechts),

4) Strafrecht, Strafverfahren und Gerichtsverfassung im Elsaß, in Lothringen, Luxemburg, den besetzten niederländischen Gebieten und in Belgien.

Mitarbeiter: LGRat Dr. Ansorge, RegRätin Hische, AGRat Dr. Geller.

6. Dr. Dörffler, Ministerialrat

Mitarbeiter: StA. Dr. Creifelds.

7. Grau, MinRat.

1) Strafprozeßordnung und prozeßrechtliche Nebengesetze,

2) Militärstrafrecht und Militärstrafgerichtsordnung sowie verwandte Gesetze,

3) Gnadenwesen, Amnestiegesetze,

4) Strafrecht, Strafverfahren und Gerichtsverfassung in den eingegliederten Ostgebieten, im Generalgouvernement und in Norwegen,

5) Kolonialstrafrecht.

Mitarbeiter: AGR. Dr. Sommer,

StA. Dr. Creifelds.
StA.

Dr. Creifelds

MR. Grau

MR. Rietzsch 
8. Dr. Eichler, MinRat.

LGRat

1) Strafvollstreckung,

2) Angelegenheiten der Internationalen Strafrechts- und

Dr. Dallinger Gefängniskommission und anderer internationaler strafrechtlicher Gesellschaften.

Mitarbeiter: LGR. Dr. Dallinger.

9. Dr. Hopp, ErbhGRat u. OLGR.

1) Erbhofrecht,

MinDirig.

2) Ländliche Entschuldung,

Dr. Vogels

3) Ländliche Siedlung und Rentengutsgesetzgebung,

4) Beobachtung und Auswertung der Rechtsprechung der Anerbengerichte,

5) Statistik des Erbhofrechts.

10. Dr. Hoyer, OLGRat

1) Rechtsangleichung in der Ostmark,

2) Rechtsangleichung in den sudetendeutschen Gebieten,

3) österreichisches und sudetendeutsches Landesstrafrecht,

4) Rechtsfragen des Protektorats Böhmen und Mähren.

Mitarbeiter: AGRat Dr. Geller.

11. Dr. Reisner, KGRat

OLGRat

Zwischenstaatliches Strafrecht (einschl. Verfahren und Vollzug), Auslieferungsrecht und sonstige zwischenstaatliche Rechtshilfe in Strafsachen (soweit Maßnahmen im Wege der Gesetzgebung oder zwischenstaatliche Abmachungen, die eine über den Einzelfall hinausgehende Regelung treffen, in Frage kommen).

12. Dr. Sommer, AGRat

1) Gebühren- und Kostengesetze,

MinDirig.

Dr. Suchomel

2) Mitarbeit bei Herrn MinRat Grau.

13. Dr. Creifelds, StA.

1) Strafregisterwesen

2) Mitarbeit bei den Herren MinRat Dr. Dörffler und MinRat Grau.

14. Dr. Ansorge, LGRat

Mitarbeit bei Herrn MinRat Rietzsch.

Dr. von Ammon

StA. Dr. Creifelds

AGRat Sommer

RegRätin Hische

15. Dr. Kümmerlein, LGRat

1) Jugendstrafrecht und Jugendstrafverfahrensrecht,

2) Mitarbeit am Jugendstrafvollzug.

zu 1. MinRat

Rietzsch

16. Dr. Dallinger, LGRat

1) Strafrecht, Strafprozeß und Strafvollstreckung des Auslandes,

2) Laufende Durchsicht und Bearbeitung des ausländischen Schrifttums auf dem Gebiete des Strafrechts, des Strafprozesses und der Strafvollstreckung,

3) Sonderaufträge des Abteilungsleiters und Mitarbeit bei Herrn MinRat Dr. Eichler.

17. Dr. Geller, AGRat

Mitarbeit bei Herrn MinRat Rietzsch und bei Herrn OLGRat Dr. Hoyer.

LGRat

Dr. Kümmerlein 


\begin{tabular}{ll}
\hline Arbeitsgebiete & Vertreter \\
\hline 18. Hische, Reg.Rätin & LGRat \\
1) Kriminalstatistik, & Dr. Ansorge \\
2) Mitarbeit bei Herrn MinRat Rietzsch. &
\end{tabular}

Der Abteilung gehören weiter an, sind aber zur Zeit zur Wehrmacht einberufen:
1. ORRat Dr. Doerner,
2. OLGRat Dr. Schäfer,
3. LGDir. Dr. Schafheutle,
4. OLGRat Dr. Rempe,
5. ORRat Dr. Merth,
6. KGRat Dr. Meeske,
7. StA. Dr. Estl,

[Stand vom Mai 1941]
8. StA. Dr. Grohmann,
9. AGRat Wüstenberg,
10. AGRat Dr. Kaniak,
11. LGRat Bargatzky,
12. LGRat Dr. Löscher,
13. LGRat Simmerding,
14. LGRat Dr. Wagner-Roemmich.

\section{Abteilung III}

(Strafrechtspflege)

Leiter: Ministerialdirektor Dr. Crohne

Vertreter: Die Unterabteilungsleiter für ihren Bereich, Ministerialdirigent Dr. Mettgenberg für die dem Abteilungsleiter unmittelbar unterstellten Referate.

Dem Abteilungsleiter unmittelbar unterstellte Referate. $\left.{ }^{*}\right)$

\begin{tabular}{ll}
\hline Arbeitsgebiete & Referenten \\
\hline
\end{tabular}

1. Generalreferat

A) a) Generalreferat für Straf- und Strafverfahrensrecht

ORR. Mielke (ausgenommen Unterabteilung III C)

b) Geschäftsverteilung und Urlaubsregelung für Abt. III (ausgenommen Unterabteilung III D) ${ }^{* *}$ )

Mitarbeiter zu a): AGR. Manske

B) a) Generalreferat für Strafvollstreckung, Gnadenwesen, Strafregistersachen, Vereinfachung der Strafrechtspflege, Amnestie, Ermittlungshilfe, Statistiken (ausgenommen Unterabteilung III C), Sammlung bedeutungsvoller Entscheidungen

b) Sonderaufträge im Generalreferat

Mitarbeiter: AGR. Manske

2. Hochverratssachen einschließlich der Gnadensachen und der Todesurteile

OLGR.

v. Schroeter Mitarbeiter:

KGR. Westphal

1) LGR. Rommel für Volksgerichtshof,

2) AGR. Vollmer für KG, OLG. Jena, Königsberg, München,

3) LGR. Dr. Fuchs für OLG. Hamburg, Kassel,

*) Sonderaufträge, die unmittelbar unter dem Abteilungsleiter zu bearbeiten sind, werden jeweils als solche gekennzeichnet.

**) Soweit es sich dabei um Angelegenheiten der Unterabteilungen handelt, ist die Gegenzeichnung des Unterabteilungsleiters einzuholen. 
4) LGR. Kruß für OLG. Hamm, Stuttgart, Wien,

5) AGR. Bruntsch für OLG. Breslau, Danzig, Dresden, Leitmeritz, Posen

3. Strafsachen wegen Wehrmittelbeschädigung

( $\S 1,2$ der VO. vom 25.XI.1939 - RGBI. I S. 2319)

Mitarbeiter: LGR. Rommel

OLGR.

v. Schroeter

EStA. Jaeger

4. Landesverratssachen einschließlich der Gnadensachen und der Todesurteile

Mitarbeiter im Todesurteilssachen: LGR. Fleischmann

5. a) Folgende Kriegsverbrechen vor den Sondergerichten:

1) Strafsachen aus $\$ 1$ der Kriegswirtschaftsverordnung vom 4. IX. 1939 (RGBl. I S. 1609),

2) Strafsachen aus $\$ \S 1-4$ der VO. gegen Volksschädlinge vom 5. IX. 1939 (RGBI.I S. 1679),

3) Strafsachen aus $\S \S 1,2$ der VO. gegen Gewaltverbrecher vom 5.XII. 1939 (RGBI. I S. 2378),

4) Strafsachen aus der VO. zum Schutz der Metallsammlung des Deutschen Volkes vom 29.III. 1940 (RGB1. I S. 565)

b) Sondergerichtssachen aus den eingegliederten Ostgebieten (auch Zichenau und Kattowitz) einschließlich der Gnadenentschließungen bei Todesurteilen

c) Sonderbehandlungen

d) Verbindungsführung zur SS einschließlich SD und zur Geheimen Staatspolizei.

Mitarbeiter zu a) und b):

1. LGR. Dierks

2. LGR. Fischer

3. LGR. Hoeller

4. LGR. Kehr

5. AGR. Pfenningsdorf

6. Verbindungsführung zur SA.

7. Sichtung der geschichtlich bedeutsamen Akten Mitarbeiter: RR. Dr. Melzer

8. Todesurteilssachen (von der Rechtskraft des Urteils bis zur Erledigung der Vollstreckung)

a) aus dem KGBez. und den OLGBez. Bamberg, Darmstadt, Dresden, Jena, Karlsruhe, Köln, Königsberg, Marienwerder, München, Nürnberg, Posen, Stuttgart, Zweibrücken

b) aus den OLGBez. Braunschweig, Breslau, Celle, Danzig, Düsseldorf, Frankfurt, Hamburg, Hamm, Kassel, Kattowitz, Kiel, Leitmeritz, Naumburg, Oldenburg, Prag, Rostock, Stettin

Mitarbeiter $z u$ a) und b):

Der vor Eintritt der Rechtskraft zuständige.
StA. Ehrhardt

MR. Krug

OStA. Joël

\section{MR. Altmeyer}

KGR. Dr. Hupperschwiller 


\section{Unterabteilung IIIA}

Leiter: Ministerialdirigent Dr. Mettgenberg

Vertreter: Ministerialrat Schmidt

\section{Sachreferate.}

Arbeitsgebiete

Referenten

1. Kirchenpolitische Strafsachen:

MR. Schmidt

a) sämtliche Strafverfahren gegen Geistliche beider Konfessionen sowie Ordensangehörige (mit Ausnahme der Devisenstrafsachen);

b) alle Strafverfahren mit kirchenpolitischem Einschlag, insbesondere Ausschreitungen gegen Geistliche, Kirchen oder Gegenstände religiöser Verehrung.

Mitarbeiter: 1) AGR. Lippert

2) LGR. Trommer.

2. a) Generalreferat für zwischenstaatliches Strafrecht und Strafverfahrensrecht, Auslieferungsrecht und sonstige zwischenstaatliche Rechtshilfe in Strafsachen (ohne Gesetzgebung - s. Abt. II -

OLGR.

Dr. v. Ammon und mit Ausnahme der unter 3 bezeichneten Sachen), insbesondere

aa) zwischenstaatliche Abmachungen in Einzelfällen, z. B. über die Einleitung von Strafverfahren, die Nachteile sowie den Austausch von Beschuldigten oder Verurteilten;

bb) Gewährung von Haftentschädigungen an Ausländer (Allgemeines und Grundsätzliches), Befreiung von der deutschen Strafgerichtsbarkeit;

b) Allgemeine Fragen der Generalgouvernements- und der tschechischen Protektoratsgerichtsbarkeit.

3. Rechtsprechung in Auslieferungssachen, Auslieferungsstatistik, Verzeichnisse von Dolmetschern und Übersetzern sowie Sonderaufgaben aus dem Arbeitsgebiet 2 nach näherer Weisung des Unterabteilungsleiters.

4. a) Internationale Einzelsachen,

b) Einzelsachen aus dem Generalgouvernement und der tschechischen Protektoratsgerichtsbarkeit

c) Einzelfälle aus der Konsulargerichtsbarkeit Mitarbeiter zu a):

1) LGR. Rindermann in den OLG.Bezirken Breslau, Danzig, Kattowitz, Königsberg, Marienwerder, Posen.

2) RR. Lauterbach für die übrigen Bezirke.

5. Strafrechtliche Fortbildung der Staatsanwälte und Richter Mitarbeiter: AGR. Manske.

KGR. Dr. Reisner

OLGR.

Dr. v. Ammon

KGR. Westphal

II. Bezirksreferate (Berlin, Nordsee, Westen, Prag)

\begin{tabular}{lll}
\hline OLG.Bezirk & Referent & Mitarbeiter \\
\hline 1. KGBez. Berlin & OLGR. Dr. Kühn & a) LGR. Kreienberg \\
& & b) LGR. Berner \\
2. Braunschweig & KGR. Dr. Hupperschwiller & - \\
3. Celle & ORR. Dr. Klemenz & LGR. Rindermann
\end{tabular}




$\begin{array}{rll}\text { 4. Darmstadt } & \text { MR. Altmeyer } & \text { LGR. Dr. Fuchs } \\ \text { 5. Düsseldorf } & \text { MR. Dr. Malzan } & \text { LGDir. Dr. Thiele } \\ \text { 6. Frankfurt } & \text { EStA. Jaeger } & \text { AGR. Bruntsch } \\ \text { 7. Hamburg } & \text { OLGR. Dr. v. Ammon } & \text { LGR. Schmerse } \\ \text { 8. Hamm } & \text { LGDir. Dr. Preiser } & - \\ \text { 9. Karlsruhe } & \text { MR. Dr. Krug } & \text { LGR. Ansorge } \\ \text { 10. Kiel } & \text { KGR. Dr. Hupperschwiller } & \text { LGR. Fleischmann } \\ \text { 11. Köln } & \text { MR. Altmeyer } & \text { OLGR. Dr. Wilkerling } \\ \text { 12. Leitmeritz } & \text { OLGR. Dr. Wilkerling } & - \\ \text { 13. Oldenburg } & \text { KGR. Dr. Hupperschwiller } & \text { LGR. Rommel } \\ \text { 14. Prag } & \text { KGR. Meyer } & \text { LGR. Kruß } \\ \text { 15. Zweibrücken } & \text { LGDir. Dr. Thiele } & \text { - }\end{array}$

\section{Unterabteilung III B}

Leiter: $\quad$ Ministerialdirigent Geh.Reg.Rat Dr. Schäfer

Vertreter: Ministerialrat Dr. Malzan

I. Sachreferate.

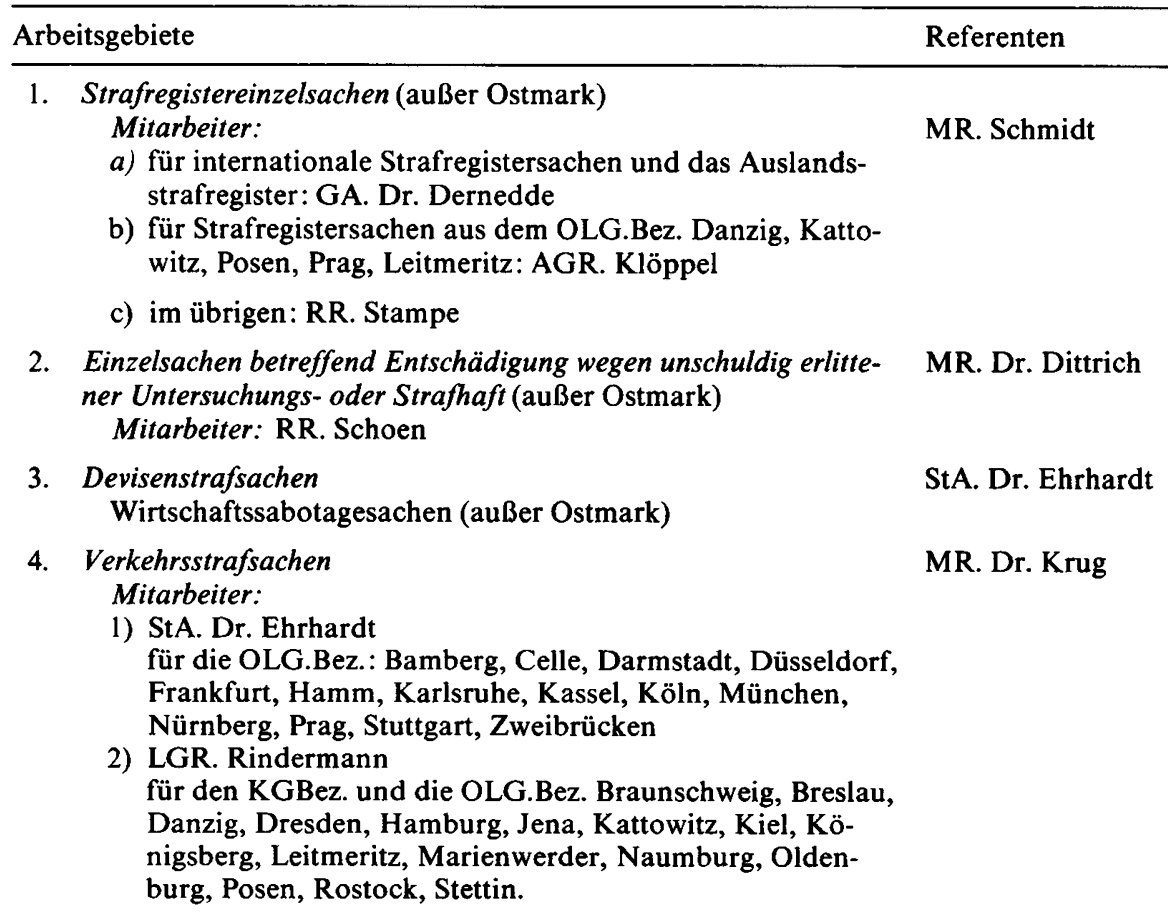


II. Bezirksreferate (Osten, Ostsee, Mittel- (außer Berlin) und Süddeutschland)

\begin{tabular}{rll}
\hline OLG.Bezirk & Referent & Mitarbeiter \\
\hline 1. Bamberg & MR. Dr. Mitzschke & LGR. Schmerse \\
2. Breslau & MR. Dr. Dittrich & AGR. Vollmer \\
3. Danzig & KGR. Meyer & LGR. Schmerse \\
4. Dresden & MR. Dr. Dittrich & LGR. Dr. Walter \\
5. Jena & MR. Dr. Malzan & LGR. Dr. Fuchs \\
6. Kassel & MR. Dr. Krug & LGR. Ansorge \\
7. Kattowitz & KGR. Meyer & LGR. Ansorge \\
8. Königsberg & KGR. Meyer & AGR. Klöppel \\
9. Marienwerder & KGR. Meyer & AGR. Klöppel \\
10. München & MR. Dr. Mitzschke & LGDir. Dr. Preiser \\
11. Naumburg & MR. Dr. Malzan & GA. Dr. Dernedde \\
12. Nürnberg & MR. Dr. Mitzschke & LGR. Dr. Fuchs \\
13. Posen & KGR. Meyer & LGR. Schmerse \\
14. Rostock & KGR. Dr. Hupperschwiller & - \\
15. Stuttgart & LGR. Kruß & - \\
16. Stettin & OLGR. Dr. v. Schroeter & LGR. Marxen
\end{tabular}

\section{Unterabteilung IIIC}

Leiter: Ministerialdirigent Dr. Suchomel

Vertreter: Ministerialdirigent Dr. Mettgenberg, f. I, 1-2

Ministerialdirigent Dr. Schäfer, f. I, 3-6

I. Sachreferate.

Arbeitsgebiete Referenten

1. Generalreferat

A) Generalreferat für Straf- und Strafverfahrensrecht in den OLGR. Hoyer Reichsgauen der Ostmark

B) Generalreferat für Gnadensachen, Strafvollstreckung,

ORR. Dr. Klemenz Strafregistersachen, Vereinfachung der Strafrechtspflege, Amnestie, Statistiken in den Reichsgauen der Ostmark

2. Todesurteilssachen (von der Rechtskraft des Urteils bis zur Erledigung der Vollstreckung)
a) aus den Bezirken Innsbruck, Linz, Wien
MR. Altmeyer

b) aus dem Bezirk Graz

Mitarbeiter zu a) und b): der vor der Rechtskraft zuständige.

KGR. Dr. Hupperschwiller

3. Strafregistereinzelsachen aus der Ostmark Mitarbeiter:

MR. Schmidt

1) StA. Elger für die OLG.Bezirke Wien, Graz.

2) AGR. Patterer für die OLG.Bezirke Linz, Innsbruck 
4. Einzelsachen betr. Entschädigung wegen unschuldig erlittener Un- MR. Dr. Dittrich tersuchungs- oder Strafhaft aus der Ostmark Mitarbeiter: RR. Schoen

5. Devisenstrafsachen, Wirtschaftssabotagesachen aus der Ostmark StA. Dr. Ehrhardt

6. Verkehrsstrafsachen aus der Ostmark Mitarbeiter: StA. Dr. Ehrhardt

MR. Dr. Krug

II. Bezirksreferate

\begin{tabular}{lll}
\hline OLG.Bezirk & Referent & Mitarbeiter \\
\hline 1. Graz & LGR. Marxen & StA. Elger \\
2. Innsbruck & LGR. Marxen & AGR. Patterer \\
3. Linz & ORR. Dr. Klemenz & StA. Elger \\
4. Wien & ORR. Dr. Klemenz & AGR. Patterer
\end{tabular}

Anhang:

Beteiligungsreferate:

Blutschutzsachen

v. Schroeter

Klosterprozesse

(Abwicklung der laufenden Sittlichkeitssachen)

Joël

Klosterprozesse

(Abwicklung der laufenden Devisensachen)

Nichtigkeitsbeschwerde

Westphal

Rundfunk (Abhören ausländischer Sender)

Westphal

Sicherstellung des Kräftebedarfs

Dittrich

Verbotener Umgang mit Kriegsgefangenen

Joël

Verfahren gegen Ausländer

v. Ammon

Kriegsverbrechen, die nicht vor den Sondergerichten angeklagt

Joël werden

Strafverfahren wegen Teilnahme an einer wehrfeindlichen Verbindung ( $\$ 3$ der Wehrkraftschutzverordnung vom 25.XI. 1939

- RGB1. I S.2319 -)

Schutzhaft, obwohl ersichtlich, daß das Gericht die Verhängung einer Haft nicht oder nicht mehr für angezeigt hält, bzw. Schutzhaft

Hupperschwiller nach Gnadenerweis

Zersetzung der Wehrkraft ( $\$ 5$ der Kriegssonderstrafrechtsverord- Meyer nung)

Luftschutzgesetz

Meyer

VO. zum Schutz gegen jugendliche Schwerverbrecher

Meyer 
Folgende Angehörige der Abteilung III einschließlich III A, B und C sind zur Wehrmacht einberufen:

1. MinRat Huber

2. OStA. von Haacke

3. OLGRat Schippert

4. KGRat Kelz

5. KGRat Jaekel

6. LGDir. Kuhn

7. EStA. Artopoeus

8. EStA. Voß

9. EStA. Carnier

10. AGRat Lorenzen

11. AGRat Siebert

12. AGRat Boenicke

13. AGRat Helmut Fritsch (Graz)

14. AGRat Durst
15. LGRat Ahlborn

16. LGRat Buri

17. LGRat Haßfurter

18. LGRat Roß

19. LGRat Blum

20. LGRat Lederer

21. LGRat Mumm

22. StA. Kutschmann

23. StA. Metz

24. StA. Petrich

25. StA. Vollmar

26. StA. Gugler

27. GAss. Stumpf.

Folgende Angehörige der Abteilung III sind abgeordnet:

a) nach Polen:

1. MinRat Wille,

2. ORR. Ganser

3. AGRat Wolf

b) nach den Niederlanden:

KGRat Seiffert

AGRat Fritsch

c) zu anderen Behörden (Stellvertreter des Führers):

MinRat Klemm

[Stand vom April 1941]

Unterabteilung IIID (Strafvollzug)

Leiter: Ministerialdirigent Marx

Vertreter: Ministerialrat Dr. Eichler

\begin{tabular}{ll}
\hline Arbeitsgebiete & Vertreter \\
\hline 1
\end{tabular}

1. Marx, Ministerialdirigent

Eichler

Allgemeine u. bezirkliche Angelegenheiten der Justizvollzugsanstalten, deren Bearbeitung sich der Leiter der Strafvollzugsabteilung von Fall zu Fall vorbehält

2. Dr. Eichler, MinRat (s. auch Abt. II)

1) Generalreferat für Vollzug (allgemeine Angelegenheiten des Vollzugs der Freiheitsstrafen, der mit Freiheitsentzie-

Hecker hung verbundenen Maßregeln der Sicherung und Besserung, der Untersuchungshaft und sonstigen Haftarten)

2) Sonderreferat für Jugendstrafvollzug und Jugendgefängnisse (in Einzelsachen mit Unterstützung seitens der jeweils zuständigen Bezirksreferenten)

3) Beteiligung an allen allgemeinen und bezirklichen Angele- Hecker genheiten von vollzugstechnischer Bedeutung

4) Gesundheitswesen der Justizvollzugsanstalten, kriminal- Hecker biologischer Dienst, Entmannung 
Vertreter

5) Beteiligung an allen allgemeinen und bezirklichen Angelegenheiten von hygienischer oder medizinischer Bedeutung

6) Beschwerden über ärztliche Behandlung und Zeichnung dieser Sachen

7) Personalsachen der Ärzte und des ärztlichen Hilfspersonals

8) Einzelsachen aus den Bezirken
a) Dresden
b) Leitmeritz
c) Prag
Müller
Hoffmann
Hoffmann

Mitarbeiter: StA. Wehl und

Hecker

der zuständige

Bezirksreferent

Hecker

\section{RegRätin Ibbeken}

3. Dr. Nörr, MinRat

1) Arbeitswesen und Arbeitseinsatz

Hoffmann

2) Beteiligung an allen allgemeinen und bezirklichen Angele- Hoffmann genheiten von Bedeutung für den Arbeitseinsatz und die Arbeitsbetriebe

3) Einzelsachen aus den Bezirken
a) Bamberg
b) Königsberg
c) Marienwerder
d) München
e) Nürnberg
f) Zweibrücken
g) Graz
h) Innsbruck
i) Linz
k) Wien

Mitarbeiter: RegRat Schulz in Angelegenheiten der allgemeinen Regelung des Arbeitswesens, RegRat Sommermeyer in den Arbeitsgebieten zu 1 und $3 \mathrm{~g}-\mathrm{k}$

4. Hecker, SenPräs.

1) Bauwesen der Justizvollzugsanstalten im allgemeinen Nörr

2) Beteiligung an Einzelbausachen von grundsätzlicher Be- Nörr deutung und soweit Ausgabemittel beansprucht werden

3) Dienstwohnungsangelegenheiten

4) Luftschutz

5) Vollstreckungsplan

6) Entlassenenfürsorge

Müller

Hoffmann

Hoffmann

Müller

Müller

Müller

Eggensperger

Eggensperger

Eggensperger

Eggensperger

7) Aus dem Generalreferat für Verwaltung der Vollzugsanstalten

a) Allgemeine Verwaltungsangelegenheiten Müller

b) Allgemeine Finanzsachen, insbes. Haushaltssachen und Müller Regelung der Personalbewilligung

8) Einzelsachen aus

a) den Bezirken
1) Breslau
2) Kattowitz
3) Stettin 
b) den Ostgebieten
1) Danzig
2) Kattowitz
3) Königsberg
4) Posen
5) Generalgouvernement
Eggensperger
Eggensperger
Eggensperger
Eggensperger
Eggensperger

Mitarbeiter: RegRat Sommermeyer in den Arbeitsgebieten zu 1 bis 5 und 7a

5. Müller, LGDir.

1) Allgemeine Besoldungssachen

2) Einzelsachen von besoldungsrechtlicher Bedeutung

3) Strafgefangenenlager im Emsland, Strafvollzug und Wachttruppe

4) Besichtigung der Justizvollzugsanstalten durch Dritte

5) Einzelsachen aus den Bezirken
a) Darmstadt
b) Frankfurt
c) Jena
d) Karlsruhe
e) Kassel
f) Naumburg
g) Stuttgart

Hecker

Hecker

Schulz

Eggensperger
Hecker
Hecker
Hoffmann
Nörr
Nörr
Hoffmann
Hoffmann

6. Hoffmann, LGDir.

1) Auswahl, Ausbildung und Fortbildung, Dienstkleidung Nörr und Bewaffnung der Strafvollzugsbeamten

2) Aus dem Generalreferat für Verwaltung der Vollzugsanstalten:

Allgemeine Beamtenangelegenheiten

Hecker

3) Einzelsachen aus den Bezirken
a) Braunschweig
b) Celle
c) Hamburg
d) Kiel
d) Oldenburg
f) Rostock

Müller

Hecker

Hecker

Eggensperger

Nörr

Müller

7. Dr. Eggensperger, EStA.

1) Hauswirtschaftssachen der Justizvollzugsanstalten Hecker

2) Hauswirtschaftsvorschriften Hecker

3) Beschaffungswesen

4) Vollzugsstatistik

Hecker

5) Unfallfürsorge für Gefangene, Unfallverhütungsvorschriften

Eichler

Müller

6) Einzelsachen aus

a) den Bezirken
1) Berlin
2) Düsseldorf
3) Hamm
4) Köln

Nörr

Hecker

b) den Westgebieten

Hecker
1) Elsaß
2) Lothringen
3) Luxemburg

Müller

Müller

Müller 


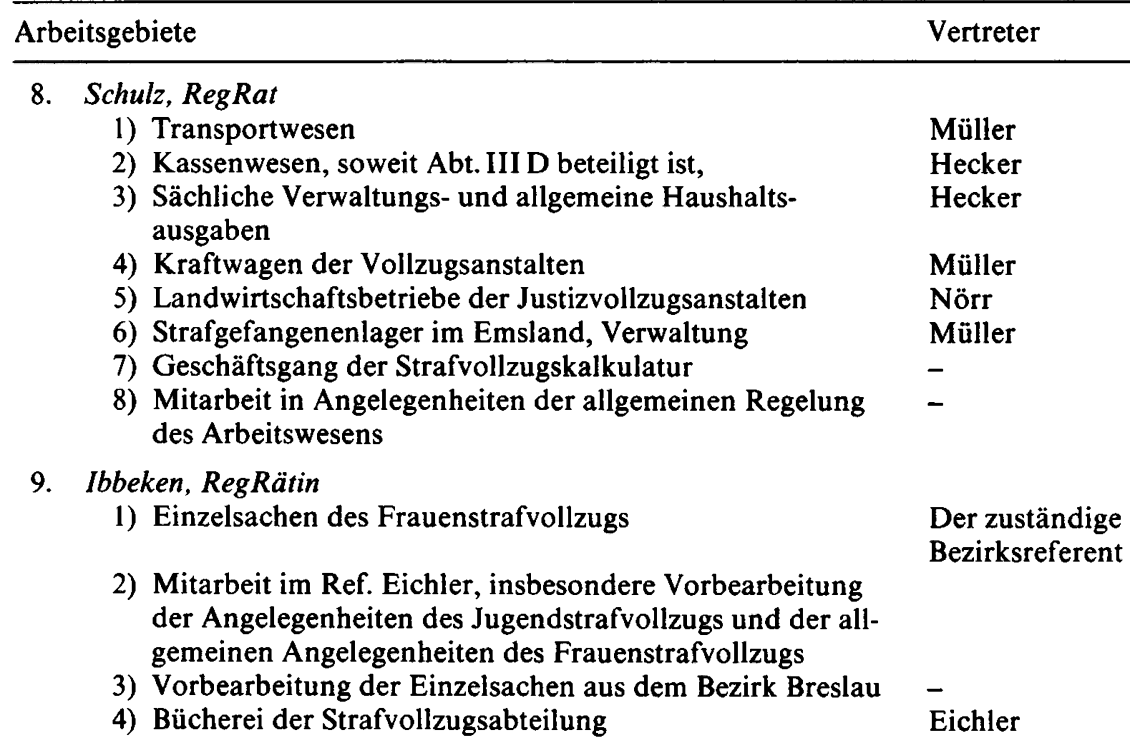

10. Sommermeyer, RegRat

Mitarbeit im Ref. Nörr und Hecker

11. Wehl, StA.

Mitarbeit im Ref. Eichler

Folgende Angehörige der Abt. IIID sind zur Wehrmacht einberufen:

1. MinRat Dr. Edgar Schmidt,

2. MinRat Dr. Weddige,

3. OStA. Thissen,

4. ORRat Dr. Merth,

5. KGRat Hey,

6. LGRat Speidel,

7. AGRat Dr. Hopf.

[Stand vom März 1941]

\section{Abteilung IV}

(Bürgerliches Recht, bürgerliche Rechtspflege; zivilrechtliche Einzelsachen, die nicht das Arbeitsgebiet der Abt.V oder das Recht der Schuldverhältnisse betreffen)

Leiter: Ministerialdirektor Dr. Volkmar

Vertreter: Ministerialdirigent Dr. Vogels

Arbeitsgebiete Vertreter

1. Dr. Vogels, MinDirigent (s. auch Abt. II)

1) Rechtserneuerung auf dem Gebiete des Erbrechts und des Konkursrechts

2) Laufende Arbeiten:

a) Erbrecht

b) Konkurs- und Vergleichsrecht, Anfechtung außerhalb des Konkurses

c) Vollstreckungsschutz für die Binnenschiffahrt Zu 2b, c:

Mitarbeiter zu 1, 2a und b: AGR. Dr. Weitnauer

Zu 2a, 3, 4, 6:

Weitnauer

Merten 
Kriegsvertretung von MinR. Dr. Richter:

3) Rechtserneuerung auf dem Gebiete des Arbeitsrechts

4) Laufende Arbeiten:

a) soziale Gesetzgebung einschl. Unfall-, Kranken-, Alters-, Invaliden- u. Angestelltenversicherung

b) materielles Arbeitsrecht

c) Erbrecht des Fiskus

Mitarbeiter zu 3 u. 4: AGR. Dr. Weitnauer

Kriegsvertretung von MinR. Dr. Pätzold:

5) Laufende Arbeiten:

Zu 5:

a) Recht der Schuldverhältnisse einschl. des Devisen- u. Aufwertungsrechts (mit Ausnahme des Miet- u. Pacht-

Breithaupt rechts)

b) Recht der Mündelsicherheit

6) Allgemeine Angelegenheiten der Akademie für Deutsches

Recht

Mitarbeiter zu 5 u. 6:

für Schuldenbereinigung, Zinssenkung, Aufwertung, Devisenrecht: AGR. Dr. Breithaupt im übrigen: AGR. Dr. Weitnauer.

2. Dr. Hesse, MinRat

1) Rechtserneuerung:

a) auf dem Gebiete des Grundbuchrechts und der freiwilligen Gerichtsbarkeit

$\mathrm{Zu} \mathrm{1,} \mathrm{3,} \mathrm{4:}$

Kaulbach

b) auf dem Gebiete des Liegenschaftsrechts (Korref. Pritsch)

c) auf dem Gebiete der allgemeinen Lehren des bürgerlichen Rechts (ausschl. des Rechts der Vereine und Stiftungen; s. Abt. V).

2) Korref. für die Rechtserneuerung auf dem Gebiete des Fahrnisrechts (Ref. Pritsch)

3) Laufende Arbeiten:

a) Grundbuchsachen

b) Angelegenheiten der freiwilligen Gerichtsbarkeit

c) Angelegenheiten der Orts- und Dorfgerichte

d) Bürgerliches Gesetzbuch, Allgemeiner Teil (ausschl. Vereine u. Stiftungen; s. Abt. V)

e) Schätzungssachen

f) Hypothekenrecht

4) Generalreferat für bürgerliches Recht in Sachen, in denen die Abt. IV nicht federführend ist Mitarbeiter: KGR. Kaulbach Kriegsvertretung von MinRat Dr. Pätzold:

5) Erneuerung des Schuldrechts (mit Ausnahme des Miet- u. Zu 5:

Pachtrechts) sowie des Hypothekenrechts

Mitarbeiter zu 5: AGR. Dr. Breithaupt

Dr. Weitnauer

AGR. Dr. Weitnauer 
4. Dr. Breithaupt, AGRat

Dr. Weitnauer

Mitarbeit im Ref. Vogels und Hesse:

a) Schuldenbereinigung, Hypothekenangelegenheiten u. Zinssenkung, Aufwertung, Devisenrecht

b) im Ref. Schultze (Einzelsachen der landwirtschaftlichen Entschuldung)

5. Dr. Weitnauer, AGRat

1) Mitarbeit im Ref. Vogels (insgesamt) und Hesse (zu 5)

Breithaupt

2) Zivilrechtliche Einzelsachen aus den Oberlandesgerichtsbezirken

Düsseldorf u. Frankfurt

6. Dr. Pritsch, MinRat

1) Rechtserneuerung auf dem Gebiete

Reinecke

a) des Fahrnisrechts (Korref. Hesse)

b) des Landpachtrechts

2) Korref. für die Rechtserneuerung auf dem Gebiete des Liegenschaftsrechts (Ref. Hesse)

3) Laufende Arbeiten auf dem Gebiete des Landpachtrechts

4) Laufende Arbeiten auf dem Gebiete des Sachenrechts, einschl. Erbbaurecht, Erbpachtrecht, Stockwerkseigentum, jedoch ohne Hypothekenrecht

5) Zivilrechtliche Einzelsachen aus den Oberlandesgerichtsbezirken

Bamberg u. Zweibrücken

Mitarbeiter zu 1, 3, 4: LGR. Reinecke

7. Reinecke, LGRat

1) Mitarbeit im Ref. Pritsch zu 1, 3, 4

2) Zivilrechtliche Einzelsachen aus den Oberlandesgerichtsbezirken

Braunschweig,

Celle,

Dresden,

Kiel,

München u.

Nürnberg

8. Maßfeller, OLGRat

1) Rechtserneuerung auf dem Gebiete des Personenstandrechts und Verwirklichung des Sippegedankens im Recht

2) Laufende Arbeiten:

a) Personenstandssachen,

b) Korref. für Jugendwohlfahrt und Fürsorgeerziehung (Ref. Dabringhaus)

c) Verhütung erbkranken Nachwuchses

d) Ehegesundheitssachen

e) Gesetzgebung zum Schutze des deutschen Blutes sowie die weitere Rassegesetzgebung (Vertretung von MinRat Dr. Ficker)

f) Familienrechtliche Befreiungen, Ehelichkeitserklärungen usw.

g) Internat. Familienrecht

Hinsichtlich der

Einzelsachen:

Merten

Rexroth 
Mitarbeiter zu 2a,c: AGR. Schlenz

$z u$ 2f: für die Befreiung von der Beibringung des ausländischen Ehefähigkeitszeugnisses: RR. Lauterbach

9. Lauterbach, RegRat (s. auch Abt. III u. V)

Mitarbeit im Ref. Maßfeller zu $2 \mathrm{f}$ für die Befreiung von der

Beibringung des ausländischen Ehefähigkeitszeugnisses

10. Rexroth, OLGRat

1) Rechtserneuerung

a) Vorarbeiten zur Erneuerung des Rechts der Wohn- und Arbeitsstätten (Miet- und gewerbliches Pachtrecht)

b) auf dem Gebiete des Rechts der deutschen Familie (Vertretung von MinRat Dr. Ficker)

2) Laufende Arbeiten auf dem Gebiete:

a) des Familienrechts

b) des Mietrechts und Pachtrechts der gewerblichen Räume

Mitarbeiter zu 2a: AGR. Schlenz

3) Korref. für Ref. Dabringhaus zu la und c

11. Dabringhaus, KGRat

1) Laufende Arbeiten

a) auf dem Gebiete der Vormundschaft über uneheliche Kinder

b) in Jugendwohlfahrt und Fürsorgeerziehung

c) im ehelichen Güterrecht

Korref. zu a und c: Rexroth zu b: Maßfeller

2) Familienrechtliche Einzelsachen

12. Dr. Schultze, KGRat

Landwirtschaftliche Entschuldung

Dabringhaus $\mathrm{u}$. Maßfeller

Mitarbeiter: AGR. Dr. Breithaupt

$\mathrm{Zu} 1 \mathrm{a}$ und $\mathrm{c}$ und 2:

Rexroth, zu lb: Maßfeller

13. Kühnemann, MinRat

1) Rechtserneuerung und laufende Arbeiten auf dem Gebiete

Sünner

a) des gewerblichen Rechtsschutzes (Recht der Patente, Gebrauchsmuster, Geschmacksmuster, Warenzeichen, Schutz gegen unlauteren Wettbewerb)

b) des literarischen und künstlerischen Urheberrechts und des Verlagsrechts

2) Angelegenheiten der Patentanwälte

3) Einzelsachen des Reichspatentamts Mitarbeiter zu 1-3: RegRat Sünner

14. Sünner, RegRat

Mitarbeit im Ref. Kühnemann zu 1-3

15. Dr. Merten, LGRat

Kriegsvertretung von LGDir. Dr. Pohle:

1) Rechtserneuerung:

a) Recht der Zwangsvollstreckung

(Beteiligung: Staud u. Stagel)

b) Recht der nichtbeamteten Rechtswahrer - mit Ausnahme der Notare - (s. auch Abt. I)

(Beteiligung: Hornig) 
2) Laufende Arbeiten:

a) Zwangsvollstreckung einschl. Zwangsversteigerung und Verwaltungszwangsverfahren (Korreferat: Stagel,

$\mathrm{Zu} 2 \mathrm{a}$ und $\mathrm{b}$ : Beteiligung: Staud)

b) zwischenstaatliches Prozeßrecht, insbesondere Verträge auf dem Gebiete der bürgerlichen Rechtspflege (Beteiligung: Staud und Stagel)

c) Anwaltsrecht (wegen der laufenden Verwaltung in Anwaltsangelegenheiten s. Abt. I) (Beteiligung: Hornig)

d) Rechtsberatungsmißbrauchgesetz: Allgemeines und Einzelsachen (Rechtsbeistände, Inkassobüros, Frachtprüfer, Versicherungsberater usw.) (Beteiligung: Hornig)

3) Beteiligung am Referat Lentz

4) Zivilrechtliche Einzelsachen aus

Hamm,

Jena,

Köln,

Königsberg,

Naumburg,

Oldenburg,

Rostock,

Stettin u.

Stuttgart

16. Staud, MinR.

1) Rechtserneuerung:

Erneuerung des Rechtsgangs in streitigen Angelegenheiten, Erkenntnisverfahren

2) Laufende Arbeiten:

a) Zivilprozeß: Erkenntnisverfahren

b) Mitwirkung bei grundsätzlichen Fragen der Justizstatistik (s. Abt. VI)

c) Korref. für Ref. Brauns zu 2a u. b

d) Beteiligung am Ref. Stagel zu $2 b$ und am Ref. Merten $\mathrm{zu}$ la und $2 \mathrm{a}$ u. $\mathrm{b}$

Mitarbeiter: LGR. Brauns

StA. Becker

Brauns

$\mathrm{Zu} 2 \mathrm{c}$ und $\mathrm{d}$ :

Hornig

$\mathrm{Zu}$ 4: Brauns

Stagel

Zu 2 u. 3: Staud

17. Dr. Stagel, MinR.

1) Rechtserneuerung:

Erkenntnisverfahren

2) Laufende Arbeiten:

a) Ostmärkisches Recht, soweit es das Arbeitsgebiet der Abt. IV betrifft

b) Generalreferat für die das Arbeitsgebiet der Abt. IV berührenden Angelegenheiten aus den Reichsgauen der Ostmark, dem Reichsgau Sudetenland und dem Protektorat Böhmen und Mähren

(Beteiligung: Staud bezüglich Gerichtsverfassung und Verfahren) 
3) Beteiligung in sämtlichen Referaten der Abt. IV bei Einführung des Reichsrechts in den Reichsgauen der Ostmark, im Reichsgau Sudetenland und im Protektorat B. u. M.

4) Korref. für Ref. Merten zu 2a

5) Beteiligung am Ref. Merten zu 1a und $2 b$

6) Zivilrechtliche Einzelsachen aus

Graz,

Innsbruck,

Leitmeritz,

Linz,

Prag u.

Wien

18. Brauns, LGRat

1) Rechtserneuerung:

Mitarbeit im Ref. Staud zu 1

2) Laufende Arbeiten:

a) Gerichtsverfassungsgesetz (Korref. Staud),

b) arbeitsgerichtliches Verfahren (Korref. Staud)

3) Zivilrechtliche Sachen aus

Breslau,

Danzig,

dem KGBezirk,

Kassel,

Posen

19. Becker, StA.

1) Rechtserneuerung:

Sammlung des für die Erneuerung des zivilprozessualen

Erkenntnisverfahrens aus Schrifttum, Rechtsprechung und Eingaben zu entnehmenden in- und ausländischen Materials

2) Laufende Arbeiten:

Mitarbeit im Ref. Staud

3) Zivilrechtliche Einzelsachen, deren Bestimmung vorbehalten bleibt

20. Reineck, LGRat (siehe auch Abt. I)

Reinecke

Zivilrechtliche Einzelsachen aus den Oberlandesgerichts-

Zu 2: Becker

Zu 3: Merten

bezirken

Darmstadt,

Karlsruhe u.

Marienwerder

21. Lentz, MinRat (s. auch Abt. I u. VII)

Gerichtsvollzieherwesen (Beteiligung: Merten)

Sebode

Mitarbeiter: AGR. Sebode

Brauns 
Vertreter

23. Hornig, KGRat (s. auch Abt. VI)

Schmidt

1) Rechtserneuerung

a) Recht der Notare (s. auch Abt. I)

b) Kostenwesen

2) Laufende Arbeiten

a) Notariatsrecht (wegen der laufenden Verwaltung in Notariatsangelegenheiten s. Abt. I)

b) Kosten- und Steuergesetzgebung (s. auch Abt. VI)

c) Gebührenabgabe der Notare (s. auch Abt. VI)

Mitarbeiter zu 1 u. 2: LGR. Dr. Schmidt $z u l b, 2 b$ : ARat Stemmler

24. Dr. Schmidt, LGRat

1) Mitarbeit im Ref. Hornig

2) Zivilrechtliche Einzelsachen aus Hamburg

hinsichtlich der

Einzelsachen

Brauns

25. Stemmler, ARat (s. auch Abt. VI)

1) Sammlung und Sichtung der das Gebiet der Abt. IV berührenden Verwaltungsanordnungen der Länder, die das Verfahren in bürgerlichen Rechtssachen betreffen

2) Mitarbeit im Ref. Hornig zu $1 \mathrm{~b}$ und $2 \mathrm{~b}$

26. Dr. Koehler, MinRat (s. auch Abt. I)

Stagel

1) Angelegenheiten der Fideikommißauflösungsbehörden,

2) Fideikommiß- und Auflösungsrecht einschl. der Einzelsachen

3) Auflösungsstiftungen und Stiftungen, die mit einem Fideikommi $ß$ und dergleichen zusammenhängen

4) Recht der Familienstiftungen einschl. der Einzelsachen

5) Adelsgesetze, Angelegenheiten der ehemaligen Landesund Standesherren

27. Witt, ARat

1) Mitarbeit im Ref. Koehler

2) Fideikommiß-Statistik

\section{Eingezogen oder abgeordnet:}

Anz, Landgerichtsrat

Düker, Amtsgerichtsrat

Epping, Amtsgerichtsrat

Fechner, Oberlandesgerichtsrat

Dr. Ficker, Ministerialrat

Henßler, Amtsgerichtsrat

Hoffmann, Kammergerichtsrat

Dr. Kramer, Amtsgerichtsrat

Dr. Pätzold, Ministerialrat

Dr. Pohle, Landgerichtsdirektor

Dr. Richter, Ministerialrat

Dr. Saage, Amtsgerichtsrat

Sarstedt, Landgerichtsrat

Dr. von Spreckelsen, Oberlandesgerichtsrat

Dr. Schäfer, Landgerichtsrat

Ziegert, Landgerichtsrat 


\section{[angegliedert:] Oberstes Fideikommißgericht}

\section{Präsident:}

Ministerialrat Dr. Hesse

stellvertr. Präsident $u$. Mitglied:

Dr. Koehler, Ministerialrat

Mitglieder:

Landforstmeister Müller-Weisker im Reichsforstamt,

MinRat Dr. Stagel im RJM.,

MinRat Otto im Reichsforstamt,

KGRat Dr. Heinemann und

ORegRat Knoetzsch im Reichsministerium für Ernährung und Landwirtschaft

stellv. Mitglieder:

MinRat Dr. Wrabec im Reichsforstamt

MinRat Bälz im RJM., Zur Militärverwaltung nach Paris abgeordnet

MinRat Dr. Weisser im Reichsministerium für Ernährung und Landwirtschaft,

LGDir. Dr. Bayr im RJM. Einberufen

Mitarbeiter nach näherer Bestimmung des Präsidenten:

Dr. Saage, Amtsgerichtsrat, Abgeordnet zum Generalgouverneur Abt. Justiz in Krakau Anz, Landgerichtsrat. Einberufen.

[Stand vom Januar 1941]

\section{Abteilung $V$}

(Handels-, Verkehrs- und Wirtschaftsrecht, Vereinsrecht, öffentliches Recht und Völkerrecht)

Leiter: Ministerialdirektor Quassowski

Vertreter: Ministerialdirigent Dr. Kriege

Arbeitsgebiete*)

Vertreter

1. Dr. Friedrich, MinRat, zum Wehrdienst einberufen.

1) Rechtserneuerung und laufende Arbeiten auf dem Gebiete des Rechts der Handelsgesellschaften (ohne die Sachen des Ref. 4 zu 1 bis 3 )

Mitarbeiter zu 1: ORegRat Geßler, LGRat Hefermehl, LGRat Dr. Meyer (einberufen)

2) Rechtserneuerung und laufende Arbeiten auf dem Gebiete des Genossenschaftsrechts und des Genossenschaftsregisters (ohne die Sachen des Ref. 6 zu 2 und 3)

Mitarbeiter zu 2: AGRat Dr. Menard

3) Korreferat für Ref.6 zu $2 \quad$ Dr. Müller

2. Krieger, MinRat

1) Seerecht und Seeschiffsregister (Beteiligung: MinRat Koffka Koffka)

Seeversicherungsrecht

Rechte an eingetragenen Schiffen (gemeinschaftlich mit MinRat Koffka)

Mitarbeiter: LGRat Dr. Hezel in Angelegenheiten des Seeschiffsregisters

OLGRat Dr. Däubler

*) Jeder Sachbearbeiter bearbeitet, soweit nichts anderes bestimmt ist, die Einzelsachen, die ihrem Gegenstande nach zu seinem Arbeitsgebiet gehören. 
2) Bank- und Sparkassenwesen

Dr. Menard

Kreditbanken

Hypothekenbanken

Schiffspfandbriefbanken

öffentlich-rechtliche

einschließlich der ein

Kreditanstalten

3) Börsenrecht

Mitarbeiter zu 2 und 3: AGRat Dr. Menard

Dr. Menard

4) Korreferat für Ref. 5 zu 1 und 2, für Ref. 6 zu 1

5) Beteiligung am Binnenschiffahrtsrecht (Ref. $7 \mathrm{zu} 1$ )

Mitarbeiter zu 1 bis 5: LGRat Dr. Weber (einberufen)

3. Dr. Müller, KGRat

1) laufende Arbeiten auf dem Gebiete des Rechts des Han-

Hefermehl delsstandes (ohne das Recht des handelsrechtlichen Arbeitsvertrages, der Handlungsagenten und der Handelsmäkler) und der Handelsgeschäfte (ohne das Recht der Spediteure, Lagerhalter und Frachtführer)

2) Internationale Vereinheitlichung des Kaufrechts

Dr. Menard

3) Schuldrechtliche Einzelsachen aus den Bezirken Danzig

Dr. Hezel und Posen

4) Mitarbeit an der Erneuerung des Rechts des Handelsstandes und der Handelsgeschäfte - ohne das Recht der Spediteure, Lagerhalter und Frachtführer -, des handelsrechtlichen Arbeitsvertrages der Handlungsagenten und der Handelsmäkler (Ref. 17 zu 2)

4. Geßler, ORegRat

1) Formblätter für Jahresabschlüsse, Wirtschaftsprüfer und

Dr. Müller Wirtschaftstreuhänder

2) Laufende Arbeiten:

Aktienrecht

Umwandlung von Kapitalgesellschaften

Bilanzerleichterungen

3) Einzelsachen auf dem Gebiete des Rechts der Handelsgesellschaften

4) Bergrechtliche Gewerkschaften

Dr. Müller

5) Schuldverschreibungsgesetz

6) Kirchenrecht

7) Kompetenzkonflikte

8) Schuldrechtliche Einzelsachen aus dem Bezirk Berlin

9) Mitarbeit an der Rechtserneuerung und den laufenden Arbeiten auf dem Gebiete des Rechts der Handelsgesellschaften (Ref. 1 zu 1)

5. Hefermehl, LGRat

1) Wirtschaftsrecht, namentlich:

Gewerberecht, Handwerksrecht

Dr. Müller

Kartellrecht

Preisrecht, Marktregelung

Vierjahresplan

Organisation der Wirtschaft

Reichswirtschaftsgericht

Judengesetzgebung

(Korref. MinRat Krieger)

Dr. Müller

Dr. Müller

Dr. Pfeifle

Dr. Pfeifle

Dr. Menard 
2) Behandlung des feindlichen Vermögens sowie Behand-

Krieger lung des norwegischen, niederländischen und belgischen Vermögens

(Korref. MinRat Krieger)

3) Laufende Arbeiten auf dem Gebiete des Rechts des handelsrechtlichen Arbeitsvertrages, der Handlungsagenten und der Handelsmäkler

4) Schuldrechtliche Einzelsachen aus den Bezirken Dresden, Karlsruhe, München, Nürnberg, Oldenburg, Stuttgart, Zweibrücken

5) Mitarbeit an der Rechtserneuerung und den laufenden Arbeiten auf dem Gebiete des Rechts der Handelsgesellschaften (Ref. 1 zu 1)

6) Mitarbeit an der Erneuerung des Rechts des Handelsstandes und der Handelsgeschäfte - ohne das Recht der Spediteure, Lagerhalter und Frachtführer -, des handelsrechtlichen Arbeitsvertrags, der Handlungsagenten und der Handelsmäkler (Ref. $17 \mathrm{zu}$ 2)

6. Dr. Menard, AGRat

1) Gesetz über die Verwahrung und Anschaffung von Wertpapieren (Korref. MinRat Krieger)

2) Gesetz über Verbrauchergenossenschaften (Korref. MinRat Dr. Friedrich)

3) Einzelsachen auf dem Gebiete des Genossenschaftsrechts und des Genossenschaftregisters

4) Schuldrechtliche Einzelsachen aus den Bezirken Breslau, Hamburg, Kassel, Kiel, Marienwerder, Naumburg, Generalgouvernement

5) Mitarbeit an der Rechtserneuerung und den laufenden Arbeiten auf dem Gebiete des Genossenschaftsrechts und des Genossenschaftsregisters (Ref. 1 zu 2)

6) Mitarbeit an Ref. 2 zu 2 und 3

7. Koffka, MinRat

1) Privates und öffentliches Verkehrsrecht (Eisenbahnen, Kraftfahrzeuge, Wasserstraßen, Binnenschiffahrt, Flößerei, Binnenschiffsregister) ohne Seerecht - s. Ref. 2 zu 1 -, ohne Luftrecht und Luftschutzrecht -s. Ref. 13 zu 2 und 3 - (Beteiligung am Binnenschiffahrtsrecht: MinRat Krieger). Rechte an eingetragenen Schiffen (gemeinschaftlich mit MinRat Krieger)

2) Recht der Spediteure, Lagerhalter und Frachtführer

3) Post- und Telegraphenrecht, Funkrecht

Mitarbeiter zu I bis 3: OLGRat Dr. Däubler

$$
\text { LGRat Dr. Hezel }
$$

4) Beteiligung am Seerecht (Ref. 2 zu 1)

5) Protektoratsangelegenheiten

Mitarbeiter zu 5: LGRat Kracht (einberufen)

Dr. Däubler

\section{Dr. Féaux de la}

Croix

Krieger

Friedrich (einberufen), Dr. Müller Friedrich (einberufen), Dr. Müller Geßler

Dr. Däubler

Dr. Däubler

Dr. Kriege

Ebersberg

1) Staats- und Verwaltungsrecht

2) Beamtenrecht (Beteiligung: MinDirig. Haastert (einberufen) vgl. Abt. I) 
3) Dienststrafrecht und Dienststrafgerichtsbarkeit (Beteiligung: MinRat Dr. Wittland vgl. Abt. I)

4) Behandlung des Vermögens der Angehörigen des ehemaligen polnischen Staats

Mitarbeiter zu I bis 4: LGRat Ebersberg

9. Dr. Mitzschke, MinRat

1) Presserecht

2) Jagd- und Forstrecht

Fischereirecht

Naturschutzwesen

10. Dr. Kriege, MinDirig.

1) Völkerrecht einschl. Kriegsrecht

Dr. Féaux de la

Croix

2) Recht der Volksgruppen

3) Staatsangehörigkeitsfragen aus internationalen Verträgen

4) Internationale Schiedsgerichte

5) Generalreferat für den Waffenstillstandsvertrag

6) Reichsgrenzsachen

7) Konsularrecht

8) Exterritorialität

9) Wehrrecht (ohne Militärstrafrecht)

10) Prisenrecht

Mitarbeiter zu l bis 10: AGRat Dr. Féaux de la Croix, LGRat Kracht (einberufen)

11. Dr. Féaux de la Croix, AGRat

1) Legalisationen (Einzelsachen s. Ref. 12)

2) Schuldrechtliche Einzelsachen aus den Bezirken Celle, Düsseldorf, Frankfurt, Hamm und Köln

Gessler

3) Mitarbeit an Ref. 10

12. Witt, ARat.

Legalisationen (Beglaubigungsvermerke)

Machule

13. Dr. Bülow, KGRat

1) Hinterlegungswesen

2) Privates und öffentliches Luftrecht

Mitarbeiter zu 1 und 2: LGRat Dr. Rinck (einberufen)

3) Luftschutzrecht

Mitarbeiter zu 3: LGRat Dr. Rinck (einberufen),

$$
\text { LGRat Dr. Seiler }
$$

Dr. Kriege

Hefermehl

4) Rechtshilfe in bürgerlichen Rechtsangelegenheiten (Allgemeines)

5) Korreferat für Ref. 15

(Breslau, Danzig, Jena, Kassel, Kiel, Königsberg, Marienwerder, Naumburg, Posen, Rostock, Stettin, Wien, Protektorat Böhmen-Mähren und Generalgouvernement)

Mitarbeiter zu 4 und 5: LGRat Dr. Stanzl (einberufen), LGRat Dr. Seiler

14. Thees, KGRat

1) Privates und öffentliches Versicherungsrecht

Dr. Bistritschan

(Sozialversicherung s. Abt. IV)

Mitarbeiter zu 1: AGRat Dr. Hagemann (einberufen)

LGRat Dr. Seiler

Dr. Kriege

Thees

Thees

Thees

Thees 
Arbeitsgebiete $\quad$ Vertreter

2) Angelegenheiten aus dem ehemaligen Heroldsamt

Dr. Kriege

Mitarbeiter zu 2: ARat Salewski (einberufen),

Reg.Rätin Dr. Melzer

3) Rechtshilfe in bürgerlichen Rechtsangelegenheiten (Allgemeines)

4) Korreferat für Ref. 15 (Bamberg, Braunschweig, Celle, Darmstadt, Dresden, Düsseldorf, Frankfurt, Graz, Hamburg, Innsbruck, Karlsruhe, Köln, Leitmeritz, Linz, München, Nürnberg, Oldenburg, Stuttgart, Zweibrücken)

Mitarbeiter zu 3 und 4: LGRat Dr. Stanzl (einberufen), LGRat Dr. Seiler

15. Salewski, ARat, zum Wehrdienst einberufen.

RegOInspekt.

1) Zwischenstaatliche Einzelsachen in Angelegenheiten des Zivilprozesses und der freiwilligen Gerichtsbarkeit (Korref. KGRat Dr. Bülow (vgl. $13 \mathrm{zu}$ 5) und KGRat Thees (vgl. $14 \mathrm{zu} 4$ ).

2) Mitarbeit an Ref. 14 zu 2

16. Dr. Däubler, OLGRat

1) Finanz- und Steuerrecht (soweit nicht Abt. VI) (Korref. Hornung MinRat Hornung)

2) Schuldrechtliche Einzelsachen aus den Bezirken Leitmeritz Dr. Pfeifle und Prag

3) Mitarbeit an Ref. 7 zu 1 bis 3

4) Mitarbeit in Angelegenheiten des Seeschiffsregisters (Ref. $2 \mathrm{zu} 1$ )

17. Dr. Bistritschan, MinRat

1) Generalreferat für die Erneuerung des Handelsrechts Krieger (ohne Gesellschaftsrecht)

2) Erneuerung des Rechts des Handelstandes und der Handelsgeschäfte - ohne das Recht der Spediteure, Lagerhalter und Frachtführer -, des handelsrechtlichen Arbeitsvertrags, der Handlungsagenten und der Handelsmäkler

Mitarbeiter zu 2: KGRat Dr. Müller, LGRat Hefermehl

3) Wechsel- und Scheckrecht

Mitarbeiter zu 3: LGRat Dr. Stanzl (einberufen)

4) Erneuerung des Rechts der Gemeinschaften (Vereine, Gesellschaften und Körperschaften) und der Stiftungen (Beteiligung: KGRat Dr. Pfeifle)

5) Korreferat für Ref. 18 zu 1 bis 7

6) Beteiligung an der Einführung des Reichsrechts in der Ostmark

18. Dr. Pfeifle, KGRat

1) Laufende Arbeiten auf dem Gebiete des Rechts der Gemeinschaften (Vereine, Gesellschaften und Körperschaften) und der Stiftungen

2) Heimstättenrecht

3) Städtisches Siedlungswesen und Städtebaugesetzgebung

4) Kleingartenrecht

Dr. Bistritschan

Dr. Bistritschan

Dr. Bistritschan

Dr. Bistritschan 
$\begin{array}{ll}\text { Arbeitsgebiete } & \text { Vertreter }\end{array}$

5) Bergrecht (ohne Recht der Gewerkschaften s. Ref. 4 zu 4)

Dr. Bistritschan

6) Wasserrecht, Wegerecht

Dr. Bistritschan

7) Landesrechtliche und öffentlichrechtliche Genossenschaften (Korref. zu 1 bis 7 MinRat Dr. Bistritschan)

8) Schuldenrechtliche Einzelsachen aus der Ostmark

Dr. Bistritschan

9) Beteiligung an der Erneuerung des Rechts der Gemein-

Dr. Däubler schaften (Vereine, Gesellschaften und Körperschaften) und der Stiftungen (Ref. 17 zu 4)

19. Hornung, MinRat

1) Kolonialrecht Beteiligt: Senatspräsident Dr. Uppenkamp

2) Korreferat für Ref. $16 \mathrm{zu} 1$

20. Dr. Uppenkamp, Sen.Präs.

Beteiligung am Kolonialrecht (Ref. 19 zu 1)

21. Kracht, LGRat, zum Wehrdienst einberufen.

Mitarbeit an Ref. 7 zu 5 und Ref. 10

22. Hagemann, AGRat, zum Wehrdienst einberufen. Mitarbeit an Ref. 14 zu 1

23. Ebersberg, LGRat

Mitarbeit an Ref. 8

24. Dr. Weber, LGRat, zum Wehrdienst einberufen. Mitarbeit an Ref. 2

25. Dr. Meyer, LGRat, zum Wehrdienst einberufen.

Mitarbeit an der Rechtserneuerung und den laufenden Arbeiten auf dem Gebiete des Rechts der Handelsgesellschaften (Ref. 1 zu 1)

26. Dr. Seiler, AGRat

Mitarbeit an Ref. 13 zu 3, 4, 5 und Ref. 14 zu 1, 3, 4

27. Dr. Stanzl, LGRat, zum Wehrdienst einberufen.

1) Mitarbeit an Ref. 13 zu 4, 5 und Ref. 14, zu 3, 4

2) Mitarbeit an Ref. 17 zu 3

28. Dr. Rinck, LGRat, zum Wehrdienst einberufen.

Mitarbeit an Ref. 13 zu 1 bis 3

29. Dr. Hezel, LGRat

1) Schuldrechtliche Einzelsachen aus den Bezirken Bamberg,

Dr. Müller

Braunschweig, Darmstadt, Jena, Königsberg, Rostock,

Stettin

2) Mitarbeit an Ref. 7 zu 1 bis 3

30. Dr. Melzer, Reg.Rätin

Mitarbeit an Ref. 14 zu 2 
[Stand vom März 1941]

\section{Abteilung VI \\ (Haushaltssachen)}

Leiter: Ministerialdirektor Schneller

Er zeichnet auch die ihm vorbehaltenen Sachen aus Abt. I und aus III D.

Vertreter: Dr. Sauer, MinDirig., und für Regreß- und Kostensachen Dr. Reichau, MinRat; im übrigen, soweit Ministerialräte beteiligt sind, der dienstälteste MinRat.

\begin{tabular}{l} 
Arbeitsgebiete \\
\hline 1. Dr. Sauer, MinDirig. (s. auch Abt. I) \\
1) Haushaltssachen des Ministeriums, des Reichsgerichts, \\
des Volksgerichtshofs und des Reichspatentamts sowie \\
der Körperschaften des öffentlichen Rechts
\end{tabular}

2) Grundstücksverwaltungs- und Bausachen:
a) der unter Nr. 1 bezeichneten Behörden, soweit sie nicht im Referat 13 bearbeitet werden,
b) aus den OLGBezirken Bamberg, Darmstadt, Graz, Innsbruck, Jena, Karlsruhe, Linz, München, Nürnberg, Stuttgart, Wien und Zweibrücken

3) Beteiligung bei Angelegenheiten von grundsätzlicher Bedeutung

a) für die Einnahmen und Ausgaben der zu 1 bezeichneten Behörden

b) bei Anwendung des 2. und 3. Rechtspflegeüberleitungsgesetzes

4) Notarkasse

5) Richterheime in Bayern

6) Justizmuseum

7) Amtstracht (Allgemeine Verwaltungsvorschriften)

Mitarbeiter zu 2: AGRat Reß und

LGRat Dümler

2. Dr. Reichau, MinRat

1) Allgemeine Besoldungsangelegenheiten

2) Besoldungs-Einzelsachen $\mathrm{Zu} 1$ und 2: soweit nicht Ref. 14 Nr. 1 Platz greift

3) Mitzeichnung der Sachen aus Ref. $14 \mathrm{Nr} .1$

4) Zeichnung der Einzelsachen (Nr. 2 und 3), denen keine grundsätzliche Bedeutung zukommt

5) Ersatzforderungen gegen den Fiskus
a) Allgemeine Angelegenheiten
b) Mitzeichnung der Sachen aus dem Referat 17 Nr. 2

3. Herwig, MinRat

1) Allgemeine Angelegenheiten der Grundstücksverwaltung und des Bauwesens

2) Einzelsachen aus den Bezirken der in Preußen gelegenen Oberlandesgerichte und der Oberlandesgerichte Braunschweig, Danzig, Dresden, Hamburg, Leitmeritz, Oldenburg, Posen, Prag und Rostock

3) Durchführung des Luftschutzes (Allgemeines)

Mitarbeiter: AGRat Reß und

LGRat Dümler
Vertreter

Für das Reichspatentamt und die Akademie f. Dt.

Recht: Dr. Schade, im übrigen Dr. Anders

Herwig

Dr. Anders

Zu 4 bis 7 :

Dr. Kirchhoff

Dr. Sandgänger

Dr. Sandgänger

fällt weg

a) Josenhan $\beta$

b) fällt weg

zu 1-3:

Dr. Sauer 
4. Zehrfeld, MinRat (s. auch Abt. I)

1) Ausarbeitung einer Geschäftsordnung für die Justizbehörden (vgl. Ref. $11 \mathrm{Nr}$. 1)

zu 1 und 2:

Sauthoff

2) Allgemeine Angelegenheiten des Geschäftsgangs (vgl. Ref. 11 Nr.2)

5. Ruppert, MinRat (s. auch Abt. I und V)

Versorgung der Beamten und ihrer Hinterbliebenen (allgemeine und Einzelsachen)

6. Dr. Anders, MinRat

1) Allgemeine Angelegenheiten des Haushalts einschl. der darauf bezüglichen Rechts- und Verwaltungsvorschriften

2) Beteiligung bei Angelegenheiten, die für die Einnahmen oder Ausgaben der Justizverwaltung von grundsätzlicher Bedeutung sind, aus allen Abteilungen (vgl. auch Ref. 1 Nr.3a)

3) Allgemeine Verwaltungssachen, für die kein anderes Referat zuständig ist

4) Alle Haushaltssachen (allgemeine und Einzelsachen), für die nicht andere Referate zuständig sind

5) Dienstkraftwagen (allgemeine Angelegenheiten und Einzelsachen Kap.4)

6) Mitzeichnung der Sachen aus Referat 14 Nr. 2-4

7. Sommer, MinRat (s. auch Abt. I)

Kraftfahrwesen des Ministeriums

Zehrfeld

zu 1, 2, 4:

Dr. Sandgänger

zu 3 und 5:

Dr. Uppenkamp

zu 6: fällt weg

Dr. Sauer

ARat Fricke

8. Osburg, ORRat (s. auch Abt. I)

Reise- und Umzugskosten, Beschäftigungsvergütungen und

Trennungsentschädigungen

1) Allgemeine Angelegenheiten

2) Einzelsachen mit Ausnahme der Einzelsachen des Ministeriums, die im Referat 9 und in Abt. I bearbeitet werden

9. Westfeld, ORRat

1) Angelegenheiten der Zahlstelle des Ministeriums einschl. Kassenaufsicht

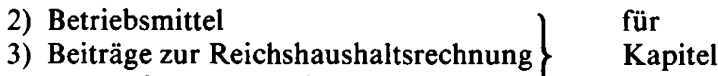

4) Vorprüfung der Rechnungslegung 1-3

5) Zeichnung der Anweisungen über die Einnahmen und über die sächlichen Ausgaben des Ministeriums

Mitarbeiter zu 5: ARat Weddigen (Zeichnung der Anweisungen über Reise- und Umzugskosten sowie über Beihilfen für den fremdsprachlichen Unterricht)

10. Sauthoff, ORRat

1) Aktenordnung

2) Geschäftsübersichten

3) Justizstatistik ohne Kriminal- und Strafvollzugsstatistik (vgl. Abt. II und III D)

4) Kalender für Reichs-Justizbeamte, Reichshandbuch, Verzeichnis der Justizbeamten des höheren Dienstes

5) Geschäftsgang der Ministerialkalkulatur (ohne die Straf- zu 5: Osburg vollzugskalkulatur)

zu 1: ARat Müller zu 2-4: Bender

zu 5:

Stadermann 
6) Mitwirkung bei der Aufstellung der Grundsätze und der zu 6: fällt weg Beschaffung der Unterlagen für die Personalbemessung (vgl. Ref. 6 Nr. 4)

7) Anträge und Beschwerden in Kostensachen aus allen Bezirken (Einzelsachen)

zu 7: Bender

11. Bender, ORRat (s. auch Abt.I)

1) Mitwirkung bei der Ausarbeitung einer Geschäftsordnung für die Justizbehörden (vgl. Ref. 4 Nr. 1)

2) Einzelsachen des Geschäftsgangs aus allen Bezirken (vgl. Ref. 4 Nr. 2)

3) Behandlung der Postsendungen einschl. Gebührenablösung

4) Büromaschinen (ohne die Kassenspezialmaschinen)

12. Stadermann, ORRat als Ministerialbürodirektor (s. auch Abt. I)

1) Geschäftsordnung und Geschäftsgang des Ministeriums, soweit nicht besondere Referate bestehen

2) Bewirtschaftung der Mittel für Anschaffungen und Bauunterhaltung des Ministeriums (Tit. 11, 12, 14-17), bei wichtigeren Angelegenheiten unter Beteiligung des Haushaltsreferenten

Mitarbeiter: ARat Andresen

13. Klee, ORRat

1) Kassen- und Rechnungswesen (allgemeine und Einzelsachen)

zu 1: fällt weg

zu 2: ARat Müller

zu 3 u. 4:

Osburg

ARat Andresen

Reg.Amtm.

Paulmann

2) Durchführung der Steuer- und Kostengesetze hinsichtlich

a) des Steuerabzugs vom Arbeitslohn

b) der Kostenentrichtung durch Kostenmarken

c) der Gebühren für die Einsicht des Schuldnerverzeichnisses

3) Durchführung der Gebührenabgabe der Notare

14. Dr. Sandgänger, ORRat

1) Die aus der Angleichung der Landesbesoldungsordnungen an die Reichsbesoldungsordnung erwachsenden Besoldungsangelegenheiten (Allgemeine und Einzelsachen)

2) Wohnungsfürsorge für die Gefolgschaft, soweit nicht die Baureferate zuständig sind

3) Haushaltssachen, die betreffen

a) die finanziellen Beziehungen der Justizverwaltung zu anderen Verwaltungen

b) die Ausstattung der Gerichte und Staatsanwaltschaften in den OLGBezirken Berlin, Braunschweig, Breslau, Celle, Danzig, Dresden, Hamburg, Kiel, Königsberg, Leitmeritz, Marienwerder, Oldenburg, Posen, Prag, Rostock und Stettin mit Plan- und Hilfsstellen

4) Ausarbeitung von Haushaltsvorschriften für die Justizverwaltung

5) Einzelaufgaben aus den Referaten 2 Nr. 1 u. $2 ; 6$

zu 5: fällt weg

15. Dr. Uppenkamp, SenPräs. (s. auch Abt. I)

Einzelaufgaben aus Referat 6

fällt weg 
16. Dr. Kirchhoff, OLGRat

1) Durchführung des Erstattungsgesetzes im allgemeinen zu 1 u. 2:

2) Kassen- und Rechnungsfehlbeträge sowie Ersatzansprüche Klee gegen Beamte und Dienstverpflichtete außerhalb der Regreßfälle (Kassenfehlbeträge unter Beteiligung des Ref. 13)

3) Einzelaufgaben aus Referat 1

zu 3: fällt weg

17. Josenhanß, KGRat

1) Vordrucke in Rechtssachen (allgemeine Angelegenheiten und Beteiligung bei der Feststellung von Vordrucken aus allen Abteilungen)

2) Ersatzforderungen gegen den Fiskus

zu 1: Bender

zu 2: Dr. Reichau

18. Hornig, KGRat (s. auch Abt.IV)

1) Durchführung der Steuer- und Kostengesetze, soweit sie nicht dem Referat 13 zugewiesen ist.

2) Kostenbefreiungen, Kostenerlasse und Bearbeitung kostenrechtlicher Bestimmungen für Abt. VI (Allgemeine Angelegenheiten)

Mitarbeiter: LGRat Dr. Schmidt und

ARat Stemmler

19. Dr. Dr. Schade, KGRat (s. auch Abt. I)

Mitarbeit im Referat 1 Nr. 1 (Reichspatentamt und Akademie für Deutsches Recht)

20. Reß, AGRat

Mitarbeit im Ref. 1 Nr. 2 und im Ref. 3

21. Dümler, LGRat

Mitarbeit im Ref. 1 Nr. 2 und im Ref. 3

zu 1 u. 2:

ARat Stemmler

fällt weg

LGRat Dümler

AGRat Reß

22. Dr. Schmidt, LGRat

Mitarbeit im Ref. 18

fällt weg

23. Weddigen, ARat

Mitarbeit im Ref. 9 Nr. 5

fällt weg

24. Andresen, ARat

Mitarbeit im Ref. 12

fällt weg

25. Stemmler, ARat

Mitarbeit im Ref. 18

fällt weg

Von den Angehörigen der Abt. VI sind zur Wehrmacht einberufen und werden für die Dauer der Einberufung nach dem vorstehenden Geschäftsverteilungsplan wie folgt vertreten:

1. Friedrich, Kurt, MinRat (s. auch Abt. I, VII)

Angelegenheiten des Gemeinschaftslagers Hanns Kerrl aus dem Geschäftsbereich der Abt. VI

Kriegsvertreter: MinRat Dr. Anders

2. Dr. Bayr, LGDir.

1) Durchführung des Erstattungsgesetzes im allgemeinen

2) Kassen- und Rechnungsfehlbeträge sowie Ersatzansprüche gegen Beamte und Dienstverpflichtete außerhalb der Regreßfälle Kriegsvertreter: OLGRat Dr. Kirchhoff 
3) Ersatzforderungen gegen den Fiskus aus den OLGBezirken Graz, Innsbruck, Leitmeritz, Linz und Wien Kriegsvertreter: KGRat Josenhan $\beta$

4) Haushaltssachen, die die Ausstattung der Gerichte und Staatsanwaltschaften mit Planstellen und Hilfskräften betreffen, aus den OLGBezirken Bamberg, Darmstadt, Düsseldorf, Frankfurt, Hamm, Jena, Karlsruhe, Kassel, Köln, München, Naumburg, Nürnberg, Stuttgart und Zweibrücken

Kriegsvertreter: MinRat Dr. Anders

5) Notarkasse Kriegsvertreter: MinDirig. Dr. Sauer

6) Mitwirkung im Ref. 6 Fällt weg

3. Dr. Bachl, OLGRat

1) Besoldungssachen aus den Bezirken der nicht in Preußen oder in der Ostmark gelegenen OLGBezirke Kriegsvertreter: MinRat Dr. Reichau

2) Mitarbeit in allgemeinen Besoldungsangelegenheiten Fällt weg

3) Durchführung des Luftschutzes (allgemeines) Kriegsvertreter: MinRat Herwig

4) Vordrucke in Rechtssachen (allg. Angelegenheiten u. Beteiligung bei der Feststellung von Vordrucken aus allen Abteilungen)

5) Ersatzforderungen gegen den Fiskus aus den OLGBezirken Bamberg, Karlsruhe, München, Nürnberg, Stuttgart und Zweibrücken

Kriegsvertreter: KGRat Josenhan $ß$

6) Bayerische Richterheime

Kriegsvertreter: MinDirig. Dr. Sauer

4. Dr. Peter, StA.

1) Mitarbeit in den Referaten 1 Nr. 1 u. 2; 5 und in den Regreßsachen aus Nr. 3 des Referats des LGDir. Dr. Bayr

2) Mitarbeit bei der Einführung von Reichsvorschriften in der Ostmark nach näherer Bestimmung des Abteilungsleiters

Fällt weg

5. Dr. Fritsch (Graz), AGRat

Kriegsvertretung des LGDir. Dr. Bayr in Regreßsachen

Erledigt durch eigene Einberufung

6. Dr. Exner, LGRat

Mitarbeit in Regreßsachen

Fällt weg

7. Dr. Gräser, LGRat

Mitarbeit im Referat 4 und in Vordruckangelegenheiten Fällt weg

8. Biermann, RegRat

1) Kostenbefreiungen, Kostenerlasse und Bearbeitung kostenrechtlicher Bestimmungen für Abt. VI (Allgemeine Angelegenheiten)

Kriegsvertreter: KGRat Hornig

2) Anträge und Beschwerden in Kostensachen aus allen Bezirken (Einzelsachen) Kriegsvertreter: ORRat Sauthoff. 
[Stand vom April 1941]

\section{Abteilung VII}

(Ausbildung)

Leiter: Präsident Dr. Palandt

Vertreter: Vizepräsident Creutzfeldt; soweit die Vertretung in den Ministerialgeschäften nicht dem Vizepräsidenten besonders übertragen ist, (s. Ref. 1) vertreten im einzelnen die jeweiligen Sachbearbeiter.

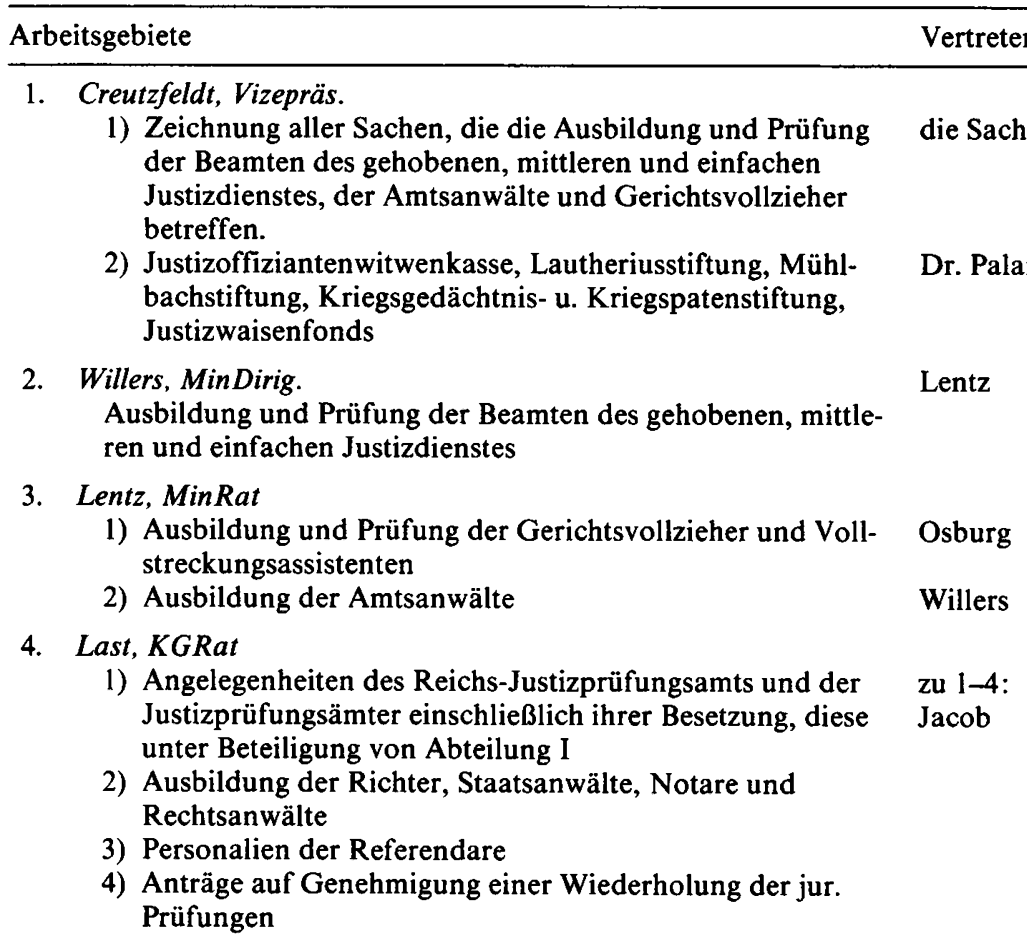

Zu den Referaten 2-4: einschließlich der Maßnahmen zur Fortbildung der festangestellten Beamten.

5. Jacob, RegRat

1) Angelegenheiten der Assessoren

2) Erlaß, Stundung und Teilzahlung von Prüfungsgebühren

3) Rückzahlung von Prüfungsgebühren

4) Anweisung der Ausgaben zu Einzelpl. IX

Kap. 1 Tit. 9

Kap. 1 Tit. 13

Kap. 1 Tit. 19

(Reichs-Justizprüfungsamt und Prüfungsstelle Berlin).

Kap. 4 Tit. 25

(Abt. VII u. Prüfungsstelle Berlin).

5) Bücherbeschaffung für Prüfungszwecke

6) Sonderaufträge des Präsidenten

zu 1, 2: Last

zu 3, 4:

Zeichnung durch den Präsidenten

Im Heeresdienst befinden sich die Sachbearbeiter MinRat Ebert, LGDir. Dr. Schumacher und AGRat Würz. 
[angegliedert:] Reichs-Justizprüfungsamt

Präsident: Dr. Palandt

Vizepräsident: Creutzfeldt

Verteilung der Verwaltungsgeschäfte

Vertreter

1. Last, KGRat

1) Genehmigung einer Wiederholung der ersten jur. Prüfung zu 1, 4 u. 5:

2) Auswahl der Aufgaben für die 5. Aufsichtsarbeit in der Jacob großen Staatsprüfung zu 2, 3:

3) Sonderaufträge bei der Auswahl von Akten

4) Aufsicht über die Prüfungsstellen des RJPA. und die Prüfungsämter (einschl. Personalsachen)

5) Nachprüfung der Niederschriften über die Prüfungen

2. Jacob, RegRat

1) Zulassung zur großen Staatsprüfung

2) Prüfungsplan

3) Einzelsachen in Prüfungsangelegenheiten

bleibt vorbehalten

4) Erteilung der Zeugnisse auch für geprüfte Amtsanwälte und Rechtspfleger

5) Verwaltungs- und Haushaltssachen des RJPA.

6) Sonderaufträge des Präsidenten

zu 1, 3 u. 4: Last

fällt fort

zu 5: ARat Kuhn fällt fort 


\title{
Anlage 3
}

\author{
Zusammenstellung \\ der in der Ära Gürtner 1933-1940 amtierenden \\ Oberlandesgerichtspräsidenten und Generalstaatsanwälte
}

\section{Oberlandesgerichtspräsidenten}

Kammergericht (Berlin):

Dr. Eduard Tigges

Heinrich Hölscher

1922-1933

1933-1943

Dr. Carl Wiechmann

Dr. Ernst Gutjahr

1931-1933

Dr. Friedrich Jung

1933

1933-1942

beim LG Berlin:

Dr. Leopold Wilde

1929-1933

Dr. Erich Thomas

1933-1936

Ernst Lautz

1936-1937

Dr. Melle Seebens

1937-1945

\section{Bamberg:}

Hans Aull

Albert Heuwieser

1932-1933

1933-1938

1938-1939

unbesetzt

(Vertreter: Vizepräsident Otto Stammler)

Dr. Ernst Dürig

1939-1944

\section{Braunschweig:}

Dr. Willy Röpcke

Dr. Bruno Heusinger

Günther Nebelung

1930-1933

1933-1934

1935-1944

Nikolaus Döll

1925-1934

(bis 1931 mit dem Titel Oberstaatsanwalt)

Otto Kahl

1934-1944

Paul Koch

1932-1933

Heinrich Müller

1933-1941

Breslau:

Max Witte

Dr. August Herwegen

Walther Frhr. von Steinaecker

1927-1933

1933-1935

1936-1942

Celle:

Adolf von Garßen

1932-1945

Georg Bach

1931-1933

Friedrich Parey

1933-1937

Karl Schnoering

1930-1933

Dr. Walter Schaeffer

1933-1935

1935-1945

Danzig:

Walter Wohler

(seit 1937 Präsident des Obergerichts

1939-1945

Danzig)

Graßmann

1939-1941

(seit 1936 Generalstaatsanwalt beim

Obergericht Danzig)

Darmstadt:

Dr. Adolf Müller

Dr. Wilhelm Stuckart

1931-1935

Fritz Hoos

Dr. Ludwig Scriba

1935

Dr. Gerhard Eckert

1928-1933

1933-1945 
Dresden:

Dr. Alfred Hüttner

Rudolf Beyer

Düsseldorf:

Dr. Franz Schollen

Wilhelm Schwister

1922-1933

1933-1943

Bernhard Wichmann

Karl Schnoering

Franz Hagemann

1932-1933

1934-1937

1937-1945

Frankfurt a. M.:

Dr. Bernhard Hempen

Otto Stadelmann

1930-1933

1933-1939

Artur Ungewitter

1939-1945

Graz:

Dr. Fritz Meldt

1938

(Kommissarischer Leiter)

1938-1945

Hamburg:

Dr. Wilhelm Kiesselbach

Dr. Arnold Engel

Dr. Curt Rothenberger

1928-1933

1933-1935

1935-1942

\section{Hamm:}

Rudolf Schneider

1933-1943

Dr. Franz Lang

Dr. Erich Drescher

1921-1933

1933-1943

Innsbruck:

Dr. Hermann Greinz

1938

(Kommissarischer Leiter)

Dr. Oskar Stritzl

1939-1945

Dr. Johann Moser

1938

(Kommissarischer Leiter der Staatsanwaltschaft)

1939-1941

Jena:

Bruno Becker

1925-1945

Dr. Werner Wurmstich

1929-1945

Karlsruhe:

Dr. Karl Buzengeiger

1930-1937

Dr. Karl Hafner

1924-1933

Heinrich Reinle

1937-1945

Emil Brettle

1933-1937

Ernst Lautz

Wilhelm Frey

1937-1939

1939-1945

Kassel:

Dr. Anz

Dr. Kurt Delitzsch

1925-1933

Dr. Erich Trautmann

1932-1945 
Kiel:

Dr. Gottfried Kuhnt

Dr. Karl Martin

Köln :

Dr. Max Josef Volmer Dr. Alexander Bergmann

1932-1933 Adolf Hepke

1928-1933

1933-1943 Paul Windhausen

1933-1943

Königsberg:

Walter Moehrs

Hugo Minde

Otto Hardt

Dr. Max Draeger

\section{Leitmeritz:}

Dr. Herbert David

1939-1944 Hermann Stein

1939-1945

Linz:

Dr. Edmund Krautmann

1939-1943

Dr. Anton Köllinger

1939-1942

Marienwerder:

Dr. Arthur Ehrhardt

Dr. Max Karge

Dr. Max Draeger

Fritz Szelinski

\section{München:}

Dr. Alexander Gerber

Georg Neithardt

1931-1933

1933-1937

Dr. Alfred Dürr

Adolf Sotier

1930-1933

1933-1934

1934-1937

1937-1943

1923-1933 Wilhelm Janßen

1932-1943

1937

1937-1943

1937-1943

beim Bayerischen Obersten Landesgericht:

Friedrich Manglkammer

1933-1935

Naumburg:

Georg Werner

Dr. Paul Sattelmacher

1923-1933 Dr. Ludwig Becker

1927-1936

1933-1945

Hermann Hahn

1936-1945

\section{Nürnberg:}

Friedrich Burkhardt

Otto Bertram

1933

1933-1937

Friedrich August Döbig

Edwin Leuchs

1926-1935

1937-1943

(bis 1931 mit dem Titel Oberstaatsanwalt)

Friedrich August Döbig 1935-1937

Dr. Emil Bems

$1937-1945$

Oldenburg:

Dr. Eduard Högl

Dr. Kurt Reuthe 
Posen:

Hellmut Froböß

1940-1945 Karl Drendel

$1940-1943$

Prag:

Fritz Bürkle

1939-1945 Helmuth Gabriel

1939-1945

Rostock:

Heinrich Burmeister

1930-1935 Paul Siegfried

Rudolf Goetsch

1935-1943 Dr. Walter Pufpaff

$-1933$

1933-1942

Stettin:

Dr. Paul Cormann

1920-1933

Dr. Gotthard Mosler

1926-1933

Richard Kulenkamp

1933-1944

Ulrich Stürenburg

1933

Dr. Reinhold Sturm

1934-1935

Otto Stäcker

1935-1945

Stuttgart:

Dr. Eugen Schmoller

1926-1933

Karl Heintzeler

1930-1937

Erwin $\mathrm{He} B$

1933-1935

Dr. Otto Küstner

1935-1945

Otto Wagner

1937-1945

Wien:

Dr. Friedrich Schober

1938

unbesetzt

1938

(Kommissarischer Leiter)

(Vertreter: Erster Oberstaats-

1938-1943 anwaltstellvertreter Dr. Brunner)

Dr. Johann Stich

1939-1945

Zweibrücken:

Friedrich Becker

Dr. Karl Siegel

1927-1933 Rudolf Troeltsch

1933-1945 Hermann Hahn

1932-1935

Heinrich Welsch

1935-1936

1936-1945 


\section{Abkürzungsverzeichnis}

\begin{tabular}{|c|c|c|c|}
\hline \multirow{3}{*}{$\begin{array}{l}\text { A.a.O. } \\
\text { ABGB }\end{array}$} & am angegebenen Ort & Dir. & Direktor \\
\hline & Allgemeines Bürgerliches & Dirig. & Dirigent \\
\hline & Gesetzbuch für Österreich & DJ & Deutsche Justiz \\
\hline Abs. & Absatz & DJZ & Deutsche Juristenzeitung \\
\hline Abt. & Abteilung & DNB & Deutsches Nachrichtenbüro \\
\hline $\begin{array}{l}\text { a. D. } \\
\text { ADO }\end{array}$ & $\begin{array}{l}\text { außer Dienst } \\
\text { Allgemeine Dienstordnung }\end{array}$ & DNSAP & $\begin{array}{l}\text { Deutsche Nationalsozialistische } \\
\text { Arbeiterpartei }\end{array}$ \\
\hline $\mathrm{AG}$ & Amtsgericht & DNVP & Deutschnationale Volkspartei \\
\hline AGR, & & DO & Dienstordnung \\
\hline AGRat & Amtsgerichtsrat & Dok. & Dokument \\
\hline AkDR & Akademie für Deutsches Recht & DR & Deutsches Recht \\
\hline Akz. & Aktenzeichen & DRiZ & Deutsche Richterzeitung \\
\hline Anm. & Anmerkung & DStR & Deutsches Strafrecht \\
\hline AR, ARat & Amtsrat & DStro & Dienststrafordnung \\
\hline Arch. & Archiv & Dt. & Deutsch \\
\hline Ausz. & Auszug & DVP & Deutsche Volkspartei \\
\hline \multirow[t]{2}{*}{ AV } & Allgemeine Verfügung & & \\
\hline & & Eintr. & Eintrag \\
\hline BA & Bundesarchiv & EK & Eisernes Kreuz \\
\hline BBG & Berufsbeamtengesetz & ErbhofGR & Erbhofgerichtsrat \\
\hline $\mathrm{BDM}$ & Bund Deutscher Mädchen & Erl. & Erlaß \\
\hline Bespr. & Besprechung & EStA & Erster Staatsanwalt \\
\hline Bez. & Bezirk & & \\
\hline BGB & Bürgerliches Gesetzbuch & FAZ & Frankfurter Allgemeine \\
\hline BGH & Bundesgerichtshof & & Zeitung \\
\hline BJM & Bundesjustizminister(ium) & FM & Finanzminister[ium] \\
\hline \multirow[t]{2}{*}{ BISchG } & $\begin{array}{l}\text { Gesetz zum Schutze des } \\
\text { deutschen Blutes und der }\end{array}$ & FS & Fernschreiben \\
\hline & $\begin{array}{l}\text { deutschen Ehre („Blutschutz- } \\
\text { gesetz“) }\end{array}$ & $\begin{array}{l}\text { G } \\
\text { GA, GAss. }\end{array}$ & $\begin{array}{l}\text { Gesetz, Gericht } \\
\text { Gerichtsassessor }\end{array}$ \\
\hline \multirow[t]{2}{*}{ BNSDJ } & Bund Nationalsozialistischer & GBl. & Gesetzblatt \\
\hline & $\begin{array}{l}\text { Deutscher Juristen } \\
\text { Bayerische Politische Polizei }\end{array}$ & GBV & $\begin{array}{l}\text { Generalbevollmächtigter für } \\
\text { die Reichsverwaltung }\end{array}$ \\
\hline BVP & Bayerische Volkspartei & GDA & $\begin{array}{l}\text { Gewerkschaftsbund der } \\
\text { Angestellten }\end{array}$ \\
\hline Ch. & Chef & $\begin{array}{l}\text { GehReg- } \\
\text { Rat }\end{array}$ & Geheimer Regierungsrat \\
\hline DAF & Deutsche Arbeitsfront & GehStP, & \\
\hline $\mathrm{DAZ}$ & Deutsche Allgemeine Zeitung & GehStPol & Geheime Staatspolizei \\
\hline DBG & Deutsches Beamtengesetz & GerO & Gerichtsordnung \\
\hline $\mathrm{DC}$ & Document Center [Berlin] & Gestapa & Geheimes Staatspolizeiamt \\
\hline DDJB & Der Deutsche Justizbeamte & Gestapo & Geheime Staatspolizei \\
\hline DDP & $\begin{array}{l}\text { Deutsche Demokratische } \\
\text { Partei }\end{array}$ & $\begin{array}{l}\text { gez. } \\
\text { GG }\end{array}$ & $\begin{array}{l}\text { gezeichnet } \\
\text { Grundgesetz }\end{array}$ \\
\hline DGWR & $\begin{array}{l}\text { Deutsches Gemein- und } \\
\text { Wirtschaftsrecht }\end{array}$ & $\mathrm{GmbH}$ & $\begin{array}{l}\text { Gesellschaft mit beschränkter } \\
\text { Haftung }\end{array}$ \\
\hline
\end{tabular}


G.P.U. Gossudarstwennoje polititscheskoje uprawlenije (Staatliche Politische Verwaltung), politische Geheimpolizei in der Sowjetunion

GRR Geheimer Regierungsrat g.Rs geheime Reichssache GS Gesetzsammlung

GStA Generalstaatsanwalt

GVBl. GuVOBl.,

GVOBI. Gesetz- und Verordnungsblatt GVG Gerichtsverfassungsgesetz

$\begin{array}{ll}\text { HA } & \text { Hauptamt } \\ \text { HJ } & \text { Hitlerjugend } \\ \text { HSSPF } & \text { Höherer SS- und Polizeiführer } \\ \text { HStArch. } & \text { Hauptstaatsarchiv }\end{array}$

i.d.F.v. in der Fassung von

IdS Inspekteur der Sicherheitspolizei

IfZ Institut für Zeitgeschichte

IMG Internationaler Militärgerichtshof

i.S. im Sinne

i.V. in Vertretung

JAB Juristische Arbeitsblätter

JAO Justizausbildungsordnung

Jb. Jahrbuch

Jb.d. Jahrbuch der Akademie für

AkDR Deutsches Recht

Jg. Jahrgang

JM Justizminister[ium]

JMB1., Justizministerialblatt

JMinBl.

JR, JRat

JuS

JerwBl.

JW

$\mathrm{JZ}$

Kap. Kapitel

KdF Kanzlei des Führers

KG Kammergericht

KGR,

KGRat

$\mathrm{KJ}$

$\mathrm{KL}, \mathrm{KZ}$

KPD

KSSVO

KStVO
Justizrat

Juristische Schulung

Justizverwaltungsblatt

Juristische Wochenschrift

Juristenzeitung

Kammergerichtsrat

Kritische Justiz

Konzentrationslager

Kommunistische Partei

Deutschlands

Kriegssonderstrafrechtsverordnung

Kriegsstrafverfahrensordnung
LA Landesamt

LG Landgericht

LGD,

LGDir. Landgerichtsdirektor

LGR,

LGRat Landgerichtsrat

LJ Landesjustiz-

LJM Landesjustizminister[ium]

LKPA Landeskriminalpolizeiamt

M, Min Minister[ium], Ministerial-

MBD Ministerialbürodirektor

MBliV. Ministerialblatt des Reichsund Preußischen Ministeriums des Innern

MdI Minister[ium] des Innern

MilStGB,

MStGB Militärstrafgesetzbuch

MilSt- Militärstrafgerichtsordnung

GerO,

MilStGO,

MStGO

MinDir. Ministerialdirektor

MinDirig. Ministerialdirigent

MinRat,

MR, MRat Ministerialrat

Mob.VO Mobilmachungsverordnung

NN "Nacht und Nebel“

NS Nationalsozialismus

NSDAP Nationalsozialistische Deutsche

Arbeiterpartei

NSFK Nationalsozialistisches Flieger-

NSKK $\quad \begin{aligned} & \text { korps } \\ & \text { Nationalsozialistisches Kraft- }\end{aligned}$

NSRB fahrerkorps

Rechtswahrerbund

NSV Nationalsozialistische Volkswohlfahrt

O Ordnung

OAR Oberamtsrichter

OBgm. Oberbürgermeister

o.D. ohne Datum

ÖR Öffentliches Recht

OKH Oberkommando des Heeres

OKW Oberkommando der Wehr-

macht

OLG Oberlandesgericht

OLGPräs. Oberlandesgerichtspräsident

OLGR,

OLGRat Oberlandesgerichtsrat

OPG Oberstes Parteigericht 
OR,

ORegR,

ORegRat,

ORR,

ORRat Oberregierungsrat

OReiA Oberreichsanwalt

OSAF Oberster SA-Führer,

Oberste SA-Führung

OStA Oberstaatsanwalt

OVG Oberverwaltungsgericht

PG Polizeigesetz

Pg. Parteigenosse

PK Partei-Kanzlei

P.O. Politische Organisation

Pol. politisch, Polizei

Pr. Präsident, Preußisch

Präs. Präsident, Präsidium

Prot. Protokoll

PVG,

PolVerwG Polizeiverwaltungsgesetz

RA Rechtsanwalt

RAD Reichsarbeitsdienst

RAnwO,

RAO Rechtsanwaltsordnung

RArbM Reichsarbeitsminister[ium]

RDB Reichsbund der Deutschen

Beamten

RdErl. Runderlaß

RdSchr. Rundschreiben

RDStO Reichsdienststrafordnung

Ref. Referat, Referent

Reg. Regierung

RegRat Regierungsrat

RFiM Reichsfinanzminister[ium]

RFSS Reichsführer SS

RFSSuChd- Reichsführer SS und Chef der

DtPol. Deutschen Polizei

RG Reichsgericht

RGBl. Reichsgesetzblatt

RGSt Entscheidungen des Reichsgericht in Strafsachen

RJF Reichsjugendführer, Reichsjugendführung

RJM Reichsjustizminister[ium]

RJP Reichs-Justizprüfungsamt

RK Reichskanzlei, Reichskanzler

RKPA Reichskriminalpolizeiamt

RM Reichsmark

RMBI. Reichsministerialblatt

RMBliV siehe MBliV

RMdI Reichsminister[ium] des Innern

RMin. Reichsminister[ium]

RRAK Reichs-Rechtsanwaltskammer
RSHA Reichssicherheitshauptamt

RStGB Reichsstrafgesetzbuch

RStH Reichsstatthalter

RT Reichstag

RuPrjM Reichs- und Preußischer[s]

Justizminister[ium]

RuPrMdI Reichs- und Preußischer[s]

Minister[ium] des Innern

RV Rundverfügung

RVerwB1. Reichsverwaltungsblatt

RW Reichswehr

RWiM Reichswirtschaftsminister[ium]

SA Sturmabteilung der NSDAP

SD Sicherheitsdienst des Reichsführers SS

SdP Sudetendeutsche Partei

SenPräs. Senatspräsident

SG Sondergericht

Sign. Signatur

SJZ Süddeutsche Juristenzeitung

SPD Sozialdemokratische Partei

Deutschlands

SS Schutzstaffel der NSDAP

Schr. Schreiben

StA Staatsanwalt

StAR Staatsanwaltschaftsrat

StArch. Staatsarchiv

St.d.F. Stellvertreter des Führers

StGB Strafgesetzbuch

StMin Staatsminister[ium]

StPO Strafprozeßordnung

Sts. Staatssekretär

StVO Strafvollstreckungsordnung,

Strafvollzugsordnung

Urt. Urteil

V. Verfügung, Vize-

VB Völkischer Beobachter

Verf. Verfasser, Verfassung,

Verfügung

Verm. Vermerk

Verw. Verwaltung

VfZ Vierteljahreshefte für Zeitgeschichte

VGB Volksgesetzbuch

VGH Volksgerichtshof

V-Männer Vertrauensmänner der Gestapo

VO Verordnung

WVHA Wirtschafts- und Verwaltungs-

Hauptamt der SS 
ZAkDR Zeitschrift der Akademie für deutsches Recht

z.b.V. zur besonderen Verwendung

ZF Zentralabteilung des Sts.

\section{Freisler}

ZfbL Zeitschrift für bayerische Landesgeschichte

ZfGWiss. Zeitschrift für Geschichtswissenschaft
ZfNRG Zeitschrift für Neuere Rechtsgeschichte

Zivilprozeßordnung

ZRP Zeitschrift für Rechtspolitik

ZS Zentralabteilung des Sts.

Schlegelberger

ZStA Zentralstaatsanwaltschaft

ZStrW, Zeitschrift für die gesamte

ZStrWiss. Strafrechtswissenschaft

z.Zt. zur Zeit 


\section{Quellen- und Literaturverzeichnis}

\section{Unveröffentlichte Quellen}

Bundesarchiv Berlin

Akten des Reichsjustizministeriums. Neue Bestandsbezeichnung: R 3001 (statt R 22); die Signaturen blieben unverändert.

\begin{tabular}{|c|c|c|c|}
\hline Signaturen 1 & R $22 / 4$ & Signaturen R 22/953-956 & Signaturen R 22/3355-3364 \\
\hline & R $22 / 12$ & R $22 / 1032$ & R $22 / 3366-3371$ \\
\hline & R 22/14 & R 22/1034-1039 & R 22/3373-3377 \\
\hline & R $22 / 21$ & R 22/1041 & R 22/3379-3382 \\
\hline & R 22/27 & R 22/1054 & R 22/3387 \\
\hline & R 22/29 & R $22 / 1056$ & R 22/3389 \\
\hline & R 22/30 & R 22/1059 & R 22/3791-3793 \\
\hline & $\mathrm{R} 22 / 50-52$ & R 22/1061 & R $22 / 4003$ \\
\hline & R $22 / 56$ & R $22 / 1085$ & R 22/4068 \\
\hline & R $22 / 58$ & R $22 / 1088$ & R 22/4091-4155 \\
\hline & R 22/59 & R 22/1089 & R $22 / 4158$ \\
\hline & R 22/131 & R $22 / 1127$ & R $22 / 4162$ \\
\hline & $\mathrm{R} 22 / 206$ & R $22 / 1138$ & R $22 / 4200$ \\
\hline & R 22/208 & R $22 / 1187$ & R 22/4209 \\
\hline & $\mathrm{R} 22 / 245$ & R $22 / 1219$ & R 22/4237 \\
\hline & R $22 / 246$ & R $22 / 1238$ & R 22/4277 \\
\hline & R $22 / 248$ & R 22/1259 & R $22 / 4278$ \\
\hline & R $22 / 253$ & R $22 / 1263$ & R 22/4430 \\
\hline & R $22 / 254$ & $\mathrm{R} 22 / 1315$ & R 22/4433 \\
\hline & $\mathrm{R} 22 / 303$ & R $22 / 1333$ & R 22/4435 \\
\hline & R 22/304 & R 22/1334 & R 22/4469 \\
\hline & R 22/603 & R 22/1337 & R 22/4470 \\
\hline & R 22/678 & R 22/1421 & R 22/4495 \\
\hline & R $22 / 683$ & R $22 / 1457$ & R 22/4502 \\
\hline & R 22/706 & R $22 / 1462$ & R 22/4553 \\
\hline & R 22/721 & R $22 / 1463$ & R 22/4594 \\
\hline & R 22/847-849 & R $22 / 1467$ & R $22 / 4690$ \\
\hline & R 22/852-856 & R 22/1469 & R 22/4693 \\
\hline & R 22/859 & R 22/1500 & R 22/4696 \\
\hline & R $22 / 862$ & R $22 / 1505$ & R $22 / 4723$ \\
\hline & R 22/863 & R $22 / 1518$ & R 22/5004 \\
\hline & R 22/865 & R $22 / 1522$ & R $22 / 5009$ \\
\hline & R 22/867 & R 22/1529 & R 22/5019-5021 \\
\hline & R 22/873 & R $22 / 1792-1794$ & R 22/5029 \\
\hline & R 22/876-878 & R $22 / 2050$ & R $22 / 5032$ \\
\hline & R $22 / 888$ & R $22 / 2076$ & R 22/5043 \\
\hline & R $22 / 928-931$ & R 22/2139 & R 22/5046 \\
\hline & R 22/941 & R 22/2740 & \\
\hline & R 22/945-947 & R 22/3168 & \\
\hline
\end{tabular}


Vorläufige Signaturen R 22/20021

R 22/20028

R 22/20040

R 22/20062

R 22/20083

R 22/20113

R 22/20312

R 22/20381

R $22 / 20383$

\author{
Vorläufige Signaturen R 22/20389 \\ R 22/20402 \\ R 22/22042 \\ R 22 Gruppe 5/316 \\ R 22 Gruppe 5/321 \\ R 22 Gruppe 5/485 \\ R 22 Gruppe 5/II-4 \\ R 22 Gruppe 5/A 10
}

Personalakten (ohne Signaturen)

Akten der Reichskanzlei

Signaturen $\mathrm{R} 43 \mathrm{I} / 1457$

R $43 \mathrm{I} / 1458$

R $43 \mathrm{I} / 1460$

R $43 \mathrm{I} / 1461$

R 43 I/1464

R $43 \mathrm{I} / 1465$

R $43 \mathrm{I} / 1468$

Signaturen R 43 II/175

R 43 II/294

R 43 II $/ 398$

R $43 \mathrm{II} / 419 \mathrm{a}$

R $43 \mathrm{II} / 420 \mathrm{a}$

R 43 II/ 423

R 43 II/425

R 43 II/427

R 43 II/446-448

R 43 II/494

R 43 II $/ 595$

R 43 II $/ 598$

R 43 II $/ 600$

R 43 II $/ 639$
Signaturen R 43 II/694

R 43 II/720

R 43 II/1196

R 43 II $/ 1202$

R 43 II $/ 1456$

R 43 II/1505

R $43 \mathrm{II} / 1508$

R 43 II/1510a

R $43 \mathrm{II} / 1511$

R 43 II/1511a

R 43 II/1511b

R 43 II/1512

R 43 II/ 1513

R 43 II/1513a
Signaturen R 43 II/1514

R 43 II/1516a

R $43 \mathrm{II} / 1517 \mathrm{~b}$

R 43 II $/ 1517 \mathrm{c}$

R 43 II $/ 1518$

R 43 II/1519

R 43 II/1531

R 43 II/1534-1536

R 43 II $/ 1544$

R 43 II $/ 1547$

R 43 II/1553a

R 43 II $/ 1560$

Akten des Reichsfinanzministeriums

Signaturen R 2/10868

R $2 / 23900$

R $2 / 23901$

R 2/23916-23918

R 2/23976-23980

R 2/24093-24097

Akten des Reichssicherheitshauptamtes

Signaturen R 58/243

R $58 / 259$

R 58/264

R 58/269 
Akten der Nationalsozialistischen Deutschen Arbeiterpartei

Signaturen NS/10

$\mathrm{NS} / 14$

$\mathrm{NS} / 36$

NS/137

\section{Bundesarchiv Koblenz}

Nachlässe

Signaturen NL/12 (Koch-Weser)

NL/171 (Saemisch)

\section{Geheimes Staatsarchiv Preußischer Kulturbesitz}

Akten des Preußischen Justizministeriums

Signaturen Rep. 84a/1603

Rep. 84a/3715

Signaturen Rep. 84a/7923

Rep. 84a/7953

Rep. 84a/4365-4383

Rep. 84a/8167

Rep. 84a/4542

Rep. $84 \mathrm{a} / 8828$

Rep. 84a/6334

Rep. 84a/12004

Rep. 84a/7908

Rep. 84a/12021

Rep. 84a/7909

Akten des Preußischen Staatsministeriums

Signaturen Rep. 90 Nr. 469

Rep. 90 Nr. 2338

Sitzungsprotokolle des Preußischen Staatsministeriums

Signatur Rep. 90 A Nr. 41

Akten des Preußischen Staatsministeriums betr. Geheime Staatspolizei

Signaturen Rep. 90 P Nr. 11

Rep. 90 P Nr. 67

Rep. 90 P Nr. 114

Akten des Preußischen Ministeriums des Innern

Signatur Rep. 77 Nr. 31 


\title{
Bayerisches Hauptstaatsarchiv
}

Akten des Bayerischen Staatsministeriums der Justiz

Signaturen MJu 10415

MJu 12003

MJu 12004

MJu 13247

MJu 13248

MJu 13293

MJu 13598
Signaturen MJu 16824-16826

MJu 16833-16835

MJu 16844-16846

MJu 16934

MJu 16997

MJu 19138

Akten des Bayerischen Staatsministeriums des Innern

Signaturen MInn 71687

MInn 71715

MInn 71719
Signaturen MInn 72438

MInn 73690

MInn 73708

Akten des Bayerischen Staatsministeriums des Äußeren

Signaturen MA 99518

MA 99519

MA 99525

MA 99526

MA 103476

MA 105256

MA 105257

MA 105448

MA 105479

MA 105482-105484

MA 105487
Signaturen MA 105490

MA 105491

MA 105494

MA 105499

MA 105617

MA 106288

MA 106301

MA 106311

MA 106672

MA 106677

MA 107200

Akten des Reichsstatthalters in Bayern

Signaturen Reichsstatthalter 38

Reichsstatthalter 63

Reichsstatthalter 65

Reichsstatthalter 219-221

\author{
Signaturen Reichsstatthalter 446 \\ Reichsstatthalter 594 \\ Reichsstatthalter 600 \\ Reichsstatthalter 611
}

(Die Signaturen MA und Reichsstatthalter sind in den Anmerkungen teilweise noch als Bestände des Geheimen Staatsarchivs zitiert, das unterdessen im Bayerischen Hauptstaatsarchiv, Abt. II, Neuere Bestände, aufgegangen ist.)

\section{Staatsarchiv München}

Akten der Staatsanwaltschaften

$\begin{array}{rr}\text { Signaturen } 1682 & \text { Signaturen } 10973 \\ 3098 & 10976 \\ 3099 & 10979 \\ 7014 & 10984 \\ 10870 & 10988\end{array}$

Akten des Oberlandesgerichts München 445

10988

Spruchkammerakten Franz Gürtner

Spruchkammerakten Georg Neithardt

Spruchkammerakten Albert Heuwieser 


\section{Niedersächsisches Staatsarchiv in Oldenburg}

Akten des Ministeriums des Innern

Signatur Best. 136 Nr. 2889

Nordrbein-Westfälisches Hauptstaatsarchiv

Akten der Gestapo(leit-)stellen Düsseldorf/Köln

Signatur RW 18/Nr. 2, 4, 15

\section{Hauptstaatsarchiv Stuttgart}

Akten des württembergischen Staatsministeriums

Signatur Best. E 130 b, Bü 1933-1941

Staatsarchiv Würzburg

Gestapo Würzburg

SD-Hauptaußenstelle Würzburg Nr. 24

\section{Archiv des Instituts für Zeitgeschichte}

I. Drucksachensammlung

Oberste Reichsbehörden

Signaturen $\mathrm{Da} 03.01$

Da 07.01

Da 27.48

Da 27.49

Da 31.01

NSDAP, Gliederungen und angeschlossene Verbände

Signaturen $\mathrm{Db} 13.01$

Db 15.02

Db 15.03

$\mathrm{Db} 20.02$

Db 23.10

$\mathrm{Db} 52.24$

Db 52.26 
SS und Polizei

Signaturen Dc 15.21

Dc 17.01

Dc 17.02

II. Fotokopiensammlung

$\begin{aligned} & \text { Signaturen } \mathrm{F} 71 / 2 \\ & \mathrm{~F} 90 \\ & \mathrm{~F} 134 / 2 \\ & \\ & \mathrm{Fa} 41 \\ & \mathrm{Fa} 100 / 11 \\ & \mathrm{Fa} 100 / 18 \\ & \mathrm{Fa} 100 / 19 \\ & \mathrm{Fa} 101\end{aligned}$

Signaturen
Fa 108
Fa 115
Fa $119 / 1$
Fa 152
Fa 183 a
Fa $183 / 1$
Fa 195
Fa 203/1-5
Fa $223 / 4$

Signaturen
Fa 248
Fa 271
Fa $285 / 6$
Fa $396 / 11$
Fa $442 / 5$
Fa $503 / 1$
Fa $506 / 11$
Fa $506 / 12$

III. Mikrofilmsammlung

Signaturen MA $3 / 7$
MA $5 / 3$
MA 108
MA 131
MA 193
MA $198 / 2$
MA $212 / 3$
MA 261

Signaturen MA 283

MA 293

MA 311

MA 340

MA 433

MA 443

MA 444/3

MA 465

Signaturen MA 1300/1

$M Z$ 9/22

MZ 17/3

$M Z 18 / 6$

MZ 75/15

MZ 75/16

MZ 81/81

IV. Gerichtsakten

Signaturen $\mathrm{Gb} 06.12$
Gb 06.20
Gb 06.129
Gb 09.02
Gb 10.01
Gb 11.06

Gf 01.01
Gf 03.03
Gf 03.13
Gf 03.28

Signaturen Gh 02.15

Gh 05.01

Signaturen Gm 07.94

Gm 07.95

Gk 03.16

Go 02.03

Go 03.02

Gl 03.10

Gm 07.06

Gs 03.01

Gm 07.19

Gw 04.02

Gm 07.37

Gm 07.40

V. Spruchkammerakten

Signatur Sp 4 
VI. Zeugenschrifttum

Signaturen ZS 51

ZS 207/IV

ZS 438

ZS 1956

ZS 2335

VIII. Nürnberger Dokumente

$\begin{array}{rcr}\text { Signaturen D-906 } & \text { Signaturen NG-949 } & \text { Signaturen NO-905 } \\ & \text { NG-981 } & \text { NO-1311 } \\ \text { NG-154 } & \text { NG-1440 } & \text { NO-1328 } \\ \text { NG-190 } & \text { NG-1566 } & \text { NO-1353 } \\ \text { NG-208 } & \text { NG-2489 } & \text { NO-1395 } \\ \text { NG-264 } & \text { NG-3278 } & \text { NO-2253 } \\ \text { NG-265 } & \text { NG-4744 } & \text { NO-2263 } \\ \text { NG-280 } & \text { NG-4798 } & \text { NO-2520 } \\ \text { NG-287 } & \text { NG-4821 } & \\ \text { NG-340 } & \text { NG-5263 } & \text { PS-615-618 } \\ \text { NG-417 } & & \text { PS-623 } \\ \text { NG-505 } & \text { NO-002 } & \text { PS-629 } \\ \text { NG-540 } & \text { NO-018 } & \text { PS-631 } \\ \text { NG-560 } & \text { NO-156 } & \text { PS-654 } \\ \text { NG-565 } & \text { NO-520 } & \text { PS-681 } \\ \text { NG-587 } & \text { NO-575 } & \text { PS-775 } \\ \text { NG-612 } & \text { NO-795 } & \text { PS-781 } \\ \text { NG-629 } & \text { NO-796 } & \text { PS-782 } \\ \text { NG-656 } & \text { NO-825 } & \text { PS-842-844 } \\ \text { NG-659 } & \text { NO-829 } & \text { PS-1969 } \\ \text { NG-747 } & \text { NO-831-834 } & \text { PS-3701 } \\ \text { NG-866 } & \text { NO-843-845 } & \text { PS-3813 } \\ \text { NG-908 } & \text { NO-895 } & \end{array}$

VIII. Akten des Nürnberger Juristenprozesses

Verteidigung für Joël

Dokumentenbuch I, Dok. Nr. 1, 4-7, 14

Dokumentenbuch II, Dok. Nr. 21, 26

Dokumentenbuch III, Dok. Nr. 63

Dokumentenbuch V, Dok. Nr. 88, 98

Stenographische Protokolle (d), Sitzung v. 28.4.1947, S. $2658 \mathrm{ff}$. 
Staatsarchiv der Freien und Hansestadt Hamburg

Akten des Hanseatischen Oberlandesgerichts Hamburg

Signaturen Best. 213-1

Document Center Berlin, jetzt: Bundesarchiv Berlin

Personalunterlagen

Partei-Correspondence

\section{Private Nacblässe}

Nachlaß Hans v. Dohnanyi, im Besitz von Frau Barbara Bayer-v. Dohnanyi Nachlaß Franz Gürtner, im Besitz von Herrn Dr. Fritz Gürtner 


\section{Veröffentlichte Quellen und Literatur}

Adam, U. D.: Judenpolitik im Dritten Reich, Düsseldorf 1979.

Adler, H. G.: Pogrome und Konzentrationslager. Das Jahrhundert der Barbarei, München 1966.

Akten der Reichskanzlei. Weimarer Republik. Boppard a. Rh., Die Kabinette Stresemann I und II, Bd. 1.: 13. August bis 6. Oktober 1923, Bd. 2.: 6. Oktober bis 30. November 1923, bearb. von K. D. Erdmann u. M. Vogt, 1978. Das Kabinett von Schleicher, 3. Dezember 1932 bis 30. Januar 1933, bearb. von A. Golecki, 1986. Die Regierung Hitler, T. 1.: 1933/34. Bd. 1.: 30. Januar bis 31. August 1933, Bd. 2.: 12. September 1933 bis 27. August 1934, bearb. von K.-H. Minuth, 1983.

Albrecht, D., H. Clausen (Hrsg.): Justiz und Drittes Reich, Sankelmark 1984.

Aly, G.: Medizin gegen Unbrauchbare, in: Aussonderung und Tod. Die klinische Hinrichtung des Unbrauchbaren, von G. Aly [u.a.], Berlin 1985, S. $9 \mathrm{ff}$.

Anatomie des SS-Staates, hrsg. von H. Buchheim, M. Broszat, H.-A. Jacobsen, H. Krausnick, Bd. 1.2., Olten, Freiburg i. Br. 1965.

Anderbrügge, K.: Völkisches Rechtsdenken. Zur Rechtslehre in der Zeit des Nationalsozialismus, Berlin 1978.

Anderson, D. L.: The Academy for German Law 1933-1944, phil. Diss. University of Michigan, New York 1987.

Anschütz, G.: Die Verfassung des Deutschen Reiches, 8. Aufl., Berlin 1928.

Die Arbeit der Sondergerichte in der Kriegszeit. Abgekürzter Bericht über die Tagung der Sondergerichtsvorsitzenden und Sachbearbeiter für die Sondergerichtsstrafsachen bei den Generalstaatsanwälten im Reichsjustizministerium am 24. Oktober 1939, hrsg. vom Reichsjustizministerium, Berlin 1939.

Arndt, I.: Das Frauenkonzentrationslager Ravensbrück, in: Studien zur Geschichte der Konzentrationslager, Stuttgart 1970, S. $99 \mathrm{ff}$.

Aronson, S.: Reinhard Heydrich und die Frühgeschichte von Gestapo und SD, Stuttgart 1971.

Bader, K. S.: Strafverteidigung vor deutschen Gerichten im Dritten Reich, in: JZ 27 (1972), S. $6 \mathrm{ff}$.

Bauer, F. J., E. Schmidt: Die bayerischen Volksgerichte 1918-1924. Das Problem ihrer Vereinbarkeit mit der Weimarer Reichsverfassung, in: ZfbL 1985, S. $494 \mathrm{ff}$.

Bay, J.: Der Preußenkonflikt 1932/33. Ein Kapitel aus der Verfassungsgeschichte der Weimarer Republik, jur. Diss. Erlangen-Nürnberg 1965.

Das Bayerische Staatsministerium der Justiz. Eine Rückschau, hrsg. vom Reichsjustizministerium, Abt. Bayern, München 1935.

Bayern in der NS-Zeit, München, Wien. Bd. 1.: Soziale Lage und politisches Verhalten der Bevölkerung im Spiegel vertraulicher Berichte, hrsg. von M. Broszat, E. Fröhlich, F. Wiesemann, 1977. Bd. 2.: Herrschaft und Gesellschaft im Konflikt, hrsg. von M. Broszat, E. Fröhlich, F. Wiesemann, 1979. Bd. 3. u. 4.: Herrschaft und Gesellschaft im Konflikt, hrsg. von M. Broszat, E. Fröhlich, A. Grossmann, 1981. Bd. 5.: Die Parteien KPD, SPD, BVP in Verfolgung und Widerstand, hrsg. von M. Broszat, H. Mehringer, 1983. Bd. 6.: Fröhlich, E.: Die Herausforderung des Einzelnen. Geschichten über Widerstand und Verfolgung, 1983.

Beer, H.: Widerstand gegen den Nationalsozialismus in Nürnberg 1933-1945, Nürnberg 1976.

Beer, U.: Versehrt, verfolgt, versöhnt. Horst Berkowitz, ein jüdisches Anwaltsleben, Essen 1979.

Bennecke, H.: Die Reichswehr und der „Röhm-Putsch“, München 1964.

Benz, W. (Hrsg.): Politik in Bayern 1919-1933. Berichte des württembergischen Gesandten Moser von Filseck, Stuttgart 1971.

Berber, F.: Zwischen Macht und Gewissen, München 1986.

Bertram, G.: Der Jurist und die „Rutenbündel des Faschismus“, in: ZRP 1983, S. $81 \mathrm{ff}$.

Best, W.: Die deutsche Polizei, Darmstadt 1940. 
Best, W.: Die Verordnung über außerordentliche Rundfunkmaßnahmen vom 1. Sept. 1939, in: DR 1939, S. $1697 \mathrm{ff}$.

Best, W.: Werdendes Polizeirecht, in: DR 1938, S. $224 \mathrm{ff}$.

Bethge, E.: Dietrich Bonhoeffer. Theologe, Christ, Zeitgenosse, München 1967.

Boberach, H. (Hrsg.): Meldungen aus dem Reich. Geheime Lageberichte des Sicherheitshauptamtes der SS 1939-1944, Neuwied, Berlin 1965.

Boberach, H. (Hrsg.): Meldungen aus dem Reich. Die geheimen Lageberichte des Sicherheitsdienstes der SS 1938-1945, Herrsching 1984.

Boberach, H. (Hrsg.): Richterbriefe. Dokumente zur Beeinflussung der deutschen Rechtsprechung 1942-1944, Boppard a. Rh. 1975.

Böckenförde, E.-W. (Hrsg.): Staatsrecht und Staatslehre im Dritten Reich, Heidelberg 1985.

Böhme: Die Vorbeugungsaufgaben der Polizei, in: DR 1936, S. $142 \mathrm{ff}$.

Bracher, K. D.: Die Auflösung der Weimarer Republik. Eine Studie zum Problem des Machtverfalls in der Demokratie, 3. Aufl., Villingen 1960.

Bracher, K. D.: Die deutsche Diktatur. Entstehung, Struktur, Folgen des Nationalsozialismus, 6.Aufl., Köln 1980.

Bracher, K. D., M. Funke, H.-A. Jacobsen (Hrsg.): Nationalsozialistische Diktatur 1933-1945, Bonn 1983.

Bracher, K. D., W. Sauer, G. Schulz: Die nationalsozialistische Machtergreifung. Studien zur Errichtung des totalitären Herrschaftssystems in Deutschland 1933/34, 2., durchges. Aufl., Köln, Opladen 1982.

Bracher, K. D.: Stufen der Machtergreifung, in: Bracher, K. D., W. Sauer, G. Schulz: Die nationalsozialistische Machtergreifung. T. 1., Frankfurt a. M., Berlin, Wien 1979.

Braun, M. Frhr. von: Von Ostpreußen bis Texas. Erlebnisse und zeitgeschichtliche Betrachtungen eines Ostdeutschen, Stollhamm 1955.

Brettle, E.: Ein Jahr Nichtigkeitsbeschwerde, in: DJ 1941, S. $561 \mathrm{ff}$.

Brettle, E.: Zusammenarbeit der Rechtsanwaltschaft und des Reichsgerichts, in: Erwin Bumke zum 65. Geburtstag, Berlin 1939, S. $188 \mathrm{ff}$.

Brinkmann, W.: Grenzen der analogen Anwendung von Strafgesetzen, in: DJ 1935, S. $1587 \mathrm{ff}$.

Brinkmann, W.: Die Ungleichheit der Strafzumessung, in: DJ 1936, S. $1653 \mathrm{ff}$.

Broszat, M.: Nationalsozialistische Konzentrationslager 1933-1945, in: Anatomie des SS-Staates, Bd. 2., Olten u. Freiburg i. Br. 1965, S. 7 ff.

Broszat, M.: Nationalsozialistische Polenpolitik 1939-1945, Stuttgart 1961.

Broszat, M.: Zur Perversion der Strafjustiz im Dritten Reich, in: VfZ 1958, S. $390 \mathrm{ff}$.

Broszat, M.: Der Staat Hitlers. Grundlegung und Entwicklung seiner inneren Verfassung, 7.Aufl., München 1978.

Broszat, M.: Zum Streit um den Reichstagsbrand, in: VfZ 1960, S. 275 ff.

Brüning, H.: Memoiren 1918-1934, Stuttgart 1970.

Bruns, H.-J.: Zur Frage der Zulässigkeit der „Beweisantizipation“ im Strafverfahren, in: DR 1940, S. $2041 \mathrm{ff}$.

Buchheim, H.: Fördernde Mitgliedschaft bei der SS, in: Gutachten des IfZ, Bd. 1., München 1958, S. $350 \mathrm{ff}$.

Buchheim, H.: Die SS - das Herrschaftsinstrument, in: Anatomie des SS-Staates, Bd. 1., Olten, Freiburg i. Br. 1965, S. $40 \mathrm{ff}$.

Buchheim, H.: Die SS in der Verfassung des Dritten Reiches, in: VfZ 1955, S. $132 \mathrm{ff}$.

Buchheit, G.: Richter in roter Robe. Freisler, Präsident des Volksgerichtshofes, München 1968.

Bumke, O.: Erinnerungen und Betrachtungen. Der Weg eines deutschen Psychiaters, München 1952.

Busch, R.: Die Staatsanwaltschaft im nationalsozialistischen Staat, in: DR 1934, S. $61 \mathrm{f}$.

Buschmann, W. [u.a.] (Hrsg.): Festschrift für Rudolf Gmür zum 70. Geburtstag, 28. Juli 1983, Bielefeld 1983.

Canter, K.: „Die Euthanasie-Morde“, in: FAZ v. 11. 6. 1963, S. 8.

Crohne, W.: Bedeutung und Aufgabe der Sondergerichte, in: DJ 1933, S. $384 \mathrm{f}$.

Dahm, G.: Zur Erneuerung des deutschen Strafverfahrens, in: DJ 1937, S. $1608 \mathrm{ff}$.

Dahm, G.: Gemeinschaft und Strafrecht, Kieler Universitätsreden, N. F. 5, Hamburg 1935.

Dahm, G. [u. a.]: Leitsätze über Stellung und Aufgaben des Richters, in: DRWs 1936, S. 123. 
Dahm, G., F. Schaffstein: Liberales oder autoritäres Strafrecht? Hamburg 1933.

Dahm, G.: Der Staatsanwalt im neuen Strafverfahren, in: DStR 1935, S. $257 \mathrm{ff}$.

Dahrendorf, R.: Bemerkungen zur sozialen Herkunft und Stellung der Richter an Oberlandesgerichten. Ein Beitrag zur Soziologie der deutschen Oberschicht, in: Hamburger Jb. Wirtschaftsu. Gesellschaftspol. 5 (1960), S. $260 \mathrm{ff}$.

Daluege, K.: Staatsanwaltschaft und Polizei in der Verbrechensbekämpfung, in: DJ 1935, S. $1846 \mathrm{ff}$.

Danckwerts, J.: Die Verwaltungsgerichtsbarkeit im nationalsozialistischen Staate, in: Deutsches Verwaltungsrecht, München 1937, S. $99 \mathrm{ff}$.

Daniels, H. (Hrsg.): Das Deutsche Beamtengesetz vom 26. Januar 1937 einschl. seiner Durchführungs- und Ausführungsvorschriften, Berlin 1937.

Debus, K. H.: Die Reichskristallnacht in der Pfalz. Schuldbewußtsein und Ermittlungen, in: Z. f. d. Gesch. d. Oberrheins 1981, S. $445 \mathrm{ff}$.

Degeners Wer ist's? 10. Ausg., Berlin 1935.

Dennewitz, B.: Einheitsstaat und Reichsverwaltung, in: R.Verw.Bl. 1935, S. $4 \mathrm{ff}$.

Deuerlein, E. (Hrsg.): Der Hitler-Putsch. Bayerische Dokumente zum 8./9. November 1923, Stuttgart 1962.

Deutsch, H. C.: Verschwörung gegen den Krieg, München 1969.

Das Deutsche Führerlexikon 1934/35, Berlin 1934.

Deutscher Juristentag 1933. 4. Reichstagung des Bundes National-Sozialistischer Deutscher Juristen e. V., Ansprachen und Vorträge, zusammengest. und bearb. von R. Schraut, Berlin 1933.

Deutscher Juristentag 1936. 5. Reichstagung des Bundes National-Sozialistischer Deutscher Juristen, hrsg. vom National-Sozialistischen Rechtswahrer-Bund, Berlin 1936.

Diels, R.: Lucifer ante portas, Stuttgart 1950.

Doeker, G., W. Steffani (Hrsg.): Klassenjustiz und Pluralismus. Festschrift für Ernst Fraenkel zum 75. Geburtstag am 26. Dezember 1973, Hamburg 1973.

Dölle, H.: Die Neugestaltung des Deutschen Bürgerlichen Rechts, in: ZAkDR 1937, S. 359 f.

Doerner, K.: Gegen unsachliche Angriffe auf die deutsche Rechtspflege! Eine notwendige Feststellung, in: DJ 1935, S. $895 \mathrm{ff}$.

Dohna, A. Graf zu: Der Münchener Hochverratsprozeß, in: DJZ 1924, S. $330 \mathrm{ff}$.

Dohna, A. Graf zu: Die Verwendung der Todesstrafe in Deutschland seit 1933, in: Monatsschr. f. Kriminalbiologie u. Strafrechtsreform 1939, S. 487.

Dokumente über die Verfolgung der jüdischen Bürger in Baden-Württemberg durch das nationalsozialistische Regime 1933-1945, im Auftrag der Archivdirektion Stuttgart bearb. von P. Sauer, T. 2., Stuttgart 1966.

Domarus, M.: Hitler. Reden und Proklamationen 1932-1945, Bd. 1. 2., München 1965.

Drewniak, B.: Poczatki ruchu hitlerowskiego na Pomorzu Zachodnim 1923-1934 (Die Anfänge der Hitler-Bewegung in Pommern), Poznan (Posen) 1962.

Düsseldorf und sein Landgericht 1820-1970, hrsg. vom Verein für Düsseldorfer Rechtsgeschichte e.V., Düsseldorf 1970.

Echterhölter, R.: Das öffentliche Recht im nationalsozialistischen Staat. Die deutsche Justiz und der Nationalsozialismus, T. 2., Stuttgart 1970.

Eckhardt, K. A.: Richteramt, in: DRWs 1936, S. $128 \mathrm{ff}$.

150 [Einhundertfünfzig] Jahre Landgericht Koblenz. Veröffentlichung der landeskundlichen Arbeitsgemeinschaft im Regierungsbezirk Koblenz e.V., Boppard a. Rh. 1970.

Eschenburg, T.: Zur Ermordung des Generals Schleicher, in: VfZ 1953, S. $71 \mathrm{ff}$.

Evers, H. U.: Kommentar zum Bonner Grundgesetz (Bonner Kommentar), Hamburg 1950.

Exner, F.: Richter, Staatsanwalt und Beschuldigter im Strafprozeß des neuen Staates, in: ZStrWiss. 1934/35, Bd. 54, S. 1 ff.

Fangmann, H. D., N. Paech (Hrsg.): Recht, Justiz und Faschismus, nach 1933 und heute, Köln 1984.

Fechenbach, F.: Die dritte bayerische Revolution, in: Baseler Nationalzeitung v. 9. 4. 1919.

Fenske, H.: Konservatismus und Rechtsradikalismus in Bayern nach 1919, Bad Homburg, Berlin, Zürich 1969.

Fieberg, G.: Justiz im nationalsozialistischen Deutschland, Hrsg.: Bundesministerium der Justiz, Köln 1984. 
Finger, A.: Einige Gedanken zur Denkschrift des Preußischen Justizministers „Nationalsozialistisches Strafrecht", in: Der Gerichtssaal 1934, S. 265 ff.

Fischer, L.: Rassenschande als strafbare Handlung, in: ZAkDR 1935, S. 537.

Fliedner, H.-J.: Die Judenverfolgung in Mannheim 1933-1945, Stuttgart 1971.

Folz, H.-E.: Staatsnotstand und Notstandsrecht, Köln, Berlin, Bonn, München 1962.

Fraenkel, E.: Der Doppelstaat, Frankfurt a. M., Köln 1974.

Fraenkel, E.: Das Dritte Reich als Doppelstaat (veröff. unter dem Pseudonym C. Jürgens), in: Sozialistische Warte 1937, Wiederabdruck in: Fraenkel, E.: Reformismus und Pluralismus, Hamburg 1973, S. $225 \mathrm{ff}$.

Fraenkel, E.: The Dual State. A Contribution to the Theory of Dictatorship, New York 1941.

Fraenkel, E.: Reformismus und Pluralismus, Materialien zu einer ungeschriebenen politischen Autobiographie, Hamburg 1973.

Fraenkel, E.: „Rule of Law“ in einer sich wandelnden Welt, in: Fraenkel, E.: Reformismus und Pluralismus, Hamburg 1973, S. $258 \mathrm{ff}$.

Fraenkel, W.: Die Nichtigkeitsbeschwerde in der Praxis, in: DR 1941, S. $2305 \mathrm{ff}$.

Frank, A.: Grenzen der Dienstaufsicht über die Richter, in: DRiZ 1933, S. 9 ff.

Frank, H.: Im Angesicht des Galgens. Deutung Hitlers und seiner Zeit auf Grund eigener Erlebnisse und Erkenntnisse, München-Gräfelfing 1953.

Frank, H. (Hrsg.): Deutsches Verwaltungsrecht, München 1937.

Frank, H. [u.a.] (Hrsg.): Grundfragen der deutschen Polizei. Bericht über die konstituierende Sitzung des Ausschusses über Polizeirecht der Akademie für Deutsches Recht am 11. Oktober 1936, Hamburg 1937.

Frank, H. (Hrsg.): Nationalsozialistische Leitsätze für ein neues deutsches Strafrecht, T. 1., Berlin 1935, T. 2., Berlin 1936.

Frank, H.: Nationalsozialistische Strafrechtspolitik, München 1939.

Frank, H. (Hrsg.): Nationalsozialistisches Handbuch für Recht und Gesetzgebung, München 1935.

Frank, H.: Rede des Reichsrechtsführers, Reichsministers Dr. Frank auf der Schlußkundgebung des Deutschen Juristentages 1936, in: DR 1936, S. $213 \mathrm{ff}$.

Frank, R.: Das Strafgesetzbuch für das Deutsche Reich, 18. Aufl., Tübingen 1931.

Frankenberg, G., F. J. Müller: Juristische Vergangenheitsbewältigung - Der Volksgerichtshof vorm BGH, in: KJ 1983, S. $145 \mathrm{ff}$.

Freisler, R.: Allgemeines zur Ehrenwahrung im Strafverfahren, in: Der Ehrenschutz im neuen deutschen Strafverfahren, H. 4., Berlin 1937, S. $27 \mathrm{f}$.

Freisler, R.: Zur Befreiung des Rechtsdenkens, in: DJ 1936, S. $1568 \mathrm{ff}$.

Freisler, R., W. Luetgebrune (Hrsg.): Denkschrift des Zentralausschusses der Strafrechtsabteilung der Akademie für Deutsches Recht über die Grundzüge eines Allgemeinen Deutschen Strafrechts, Berlin 1934.

Freisler, R., F. Grau, K. Krug, O. Rietzsch (Hrsg.): Deutsches Strafrecht. Bd. 1. Erläuterungen zu den seit dem 1. 9. 1939 ergangenen strafrechtlichen und strafverfahrensrechtlichen Vorschriften, Berlin 1941.

Freisler, R.: Dringende Fragen der deutschen Strafgerichtsorganisation und des deutschen Strafverfahrens, in: DStR 1936, S. $305 \mathrm{ff}$.

Freisler, R. [u. a.] (Hrsg.): Der Ehrenschutz im neuen deutschen Strafverfahren, Berlin 1937.

Freisler, R.: Die Einheit von Partei und Staat in der Personalpolitik der Justiz, in: DJ 1935, S. $1685 \mathrm{f}$.

Freisler, R.: Einige Gedanken zur Erneuerung des deutschen Strafrechts, in: DGWR vom 15.3. 1937, S. 89.

Freisler, R.: Fragen zur Sicherungsverwahrung, in: DJ 1938, S. $626 \mathrm{ff}$.

Freisler, R: : Des Führers Tat und unsere Pflicht, in: DJ 1934, S. $850 \mathrm{f}$.

Freisler, R.: Gedanken zum Gesetz gegen das räuberische Stellen von Autofallen, in: DJ 1939, S. $34 \mathrm{ff}$.

Freisler, R.: Gedanken zum Kriegsstrafrecht und zur Gewaltverbrecherverordnung, in: DJ 1939, S. $1849 \mathrm{ff}$.

Freisler, R.: Gedanken zum rechten Strafmaß, in: DStR 1939, S. $329 \mathrm{ff}$.

Freisler, R.: Gedanken zur Verordnung gegen Volksschädlinge, in: DR 1939, S. $1450 \mathrm{ff}$. 
Freisler, R.: Gemeinnutz vor Eigennutz als volksgenössische Rechtspflicht, in: DGWR 1940, S. $169 \mathrm{ff}$.

Freisler, R.: Das Gesetz zur Änderung von Vorschriften des allgemeinen Strafverfahrens, des Wehrmachtsverfahrens und des Strafgesetzbuches und seine Stellung in der Strafverfahrenserneuerung, in: DJ 1939, S. $1565 \mathrm{ff}$.

Freisler, R.: Grundzüge des kommenden Strafverfahrensrechts, in: DStR 1935, S. $228 \mathrm{ff}$.

Freisler, R.: Justiz und Politik, in: 200 Jahre Dienst am Recht, Berlin 1938, S. $191 \mathrm{ff}$.

Freisler, R.: Nationalsozialistisches Recht und Rechtsdenken, Berlin 1938.

Freisler, R.: Nationalsozialistisches Strafrecht und aufbauende Kritik, in: DJ 1934, S. 223 f.

Freisler, R.: Neue Grundsātze für die Auslese der Rechtswahrer, in: DJ 1935, S. 583 ff.

Freisler, R.: Die neue Methode der strafgerichtlichen Zuständigkeitsbestimmung, in: DJ 1940, S. $281 \mathrm{ff}$.

Freisler, R.: Das neue Strafrecht als nationalsozialistisches Bekenntnis, in: Gürtner, F., R. Freisler: Das neue Strafrecht. Grundsätzliche Gedanken zum Geleit, 2. Aufl., Berlin 1936, S. $33 \mathrm{ff}$.

Freisler, R.: Nichtigkeitsbeschwerde, in: DJ 1940, S. $341 \mathrm{ff}$.

Freisler, R.: Nochmals: Verdunkelung und Dunkelheit, in: DJ 1940, S. $1045 \mathrm{ff}$.

Freisler, R.: Personalpolitik im höheren Justizdienst, volkspolitisch gesehen, in: DJ 1939, S. $1342 \mathrm{ff}$.

Freisler, R.: Recht, Richter und Gesetz, in: DJ 1933, S. $694 \mathrm{ff}$.

Freisler, R.: Rechtspolitische Gedanken zur Wiederaufnahme des Verfahrens, in: DJ 1937, S. $730 \mathrm{ff}$.

Freisler, R.: Ein Reich - ein Recht! Gedanken zur Verschmelzung des ostmärkischen und altreichsdeutschen Strafrechts, in: DJ 1941, S. $478 \mathrm{ff}$.

Freisler, R.: Richter und Gesetz, in: Lammers, H. H., H. Pfundtner (Hrsg.): Die Verwaltungsakademie. Ein Handbuch für den Beamten im nationalsozialistischen Staat, Berlin 1934 ff., Bd. I., Gruppe 2, Beitrag 17.

Freisler, R.: Trennung, Abschied von Hanns Kerrl? in: DJ 1934, S. 787 f.

Freisler, R.: Die Vereinfachungsverordnung als Mittel der Schlagkraftsteigerung der Strafrechtspflege und ihr Stand in der Strafverfahrenserneuerung, in: DJ 1939, S. $1537 \mathrm{ff}$.

Freisler, R.: Zur Verordnung über außerordentliche Rundfunkmaßnahmen, in: DJ 1940, S. $105 \mathrm{ff}$.

Freisler, R.: Der Volksgerichtshof - das Reichsstrafgericht? in: ZAkDR 1935, S. $90 \mathrm{ff.}$

Freisler, R.: Der Volksrichter in der neuen deutschen Strafrechtspflege, Berlin 1937.

Freisler, R.: Der Weg zum Strafrechtsausschuß, in: DJ 1933, S. 623 f.

Freisler, R.: Zweikampf, in: Gürtner, F. (Hrsg.): Das kommende deutsche Strafrecht. Besonderer Teil, 2.Aufl., Berlin 1936, S. $547 \mathrm{ff}$.

Frick, W.: Die Gesetze gegen die Neubildung von Parteien und zur Sicherung der Einheit von Partei und Staat, in: Nationalsozialistisches Handbuch für Recht und Gesetzgebung, München 1934, S. $389 \mathrm{ff}$.

Friedrich, J.: Freispruch für die Nazi-Justiz. Die Urteile gegen NS-Richter seit 1948. Eine Dokumentation, Reinbek b. Hamburg 1983.

Friedrich, K.: Das Gemeinschaftslager Hanns Kerrl, in: DJ 1936, S. 759 ff.

Fritzsche, H.: Reichsminister Dr. h. c. Gürtner, in: Unsere Reichsregierung, Berlin 1936, S. $153 \mathrm{ff}$.

Gauweiler, O.: Rechtseinrichtung und Rechtsaufgaben der Bewegung, München 1939.

Geigenmüller, O.: Die politische Schutzhaft im nationalsozialistischen Staat, Würzburg 1937.

Gemmingen, Frhr. v.: Über Grundgedanken und Tragweite des Autofallengesetzes, in: DStR 1939, S. $1 \mathrm{ff}$.

Georg, E.: Die wirtschaftlichen Unternehmungen der SS, Stuttgart 1963.

Gerland, H.: Einige Anmerkungen zu der Denkschrift des Preußischen Justizministers, in: DJ 1934, S. $224 \mathrm{ff}$.

Gerland, H.: Der Strafprozeß im autoritären Staat, in: DRiZ 1933, S. 233 f.

Gisevius, H. B.: Bis zum bitteren Ende, Bd. 1., Zürich 1946.

Gleispach, W. Graf von: Die jüngsten Strafrechtsnovellen Danzigs vor dem Permanenten Gerichtshof für internationales Recht in Haag, in: DR 1936, S. $23 \mathrm{ff}$.

Gleispach, W. Graf von: Tötung, in: Gürtner, F. (Hrsg.): Das kommende deutsche Strafrecht. Besonderer Teil, 2. Aufl., Berlin 1936, S. $371 \mathrm{ff}$. 
Godau-Schüttke, K. D.: Rechtsverwalter des Reichs, Staatssekretär Dr. Curt Joel, Frankfurt a. M. 1981.

Goedel: Der $§ 2$ StGB neuer Fassung in der Rechtsprechung, in: DJ 1938, S. $587 \mathrm{ff}$.

Göppinger, H.: Die Verfolgung der Juristen jüdischer Abstammung durch den Nationalsozialismus, Villingen/Schwarzwald 1963.

Göring, H.: Reden und Aufsätze, München 1938.

Golecki, A.: Das Kabinett von Schleicher und das Ende der Weimarer Republik, in: Akten der Reichskanzlei. Das Kabinett von Schleicher, Boppard a. Rh. 1986, S. 19 ff.

Gollert, F.: Dibelius vor Gericht, München 1959.

Gordon, H. J.: Hitlerputsch 1923. Machtkampf in Bayern 1923-1924, Frankfurt a. M. 1971.

Graf, Ch.: Politische Polizei zwischen Demokratie und Diktatur. Die Entwicklung der preußischen Politischen Polizei vom Staatsschutzorgan der Weimarer Republik zum Geheimen Staatspolizeiamt des Dritten Reiches, Berlin 1983.

Graml, H.: Der 9. November 1938. „Reichskristallnacht“, Bonn 1958.

Graß, K. M.: Edgar Jung. Papenkreis und Röhmputsch 1933/34, phil. Diss. Heidelberg 1966.

Grau, F.: Die Wehrmittelbeschädigung und die unterlassene Anzeige nach dem Gesetz zur Änderung des Strafgesetzbuchs vom 2. Juli 1936, in: DJ 1936, S. $1002 \mathrm{ff}$.

Gribbohm, G.: Richter und Strafrechtspflege im Dritten Reich, in: Albrecht, D., H. Clausen (Hrsg.): Justiz und Drittes Reich, Sankelmark 1984, S. $15 \mathrm{ff}$.

Gribbohm, G.: Der Volksgerichtshof, in: JuS 1969, S. 55 ff. u. $109 \mathrm{ff}$.

Grieser, U.: Himmlers Mann in Nürnberg. Der Fall Benno Martin: Eine Studie zur Struktur des Dritten Reiches in der „Stadt der Reichsparteitage“, Nürnberg 1974.

Grimm, F.: Die Danziger Strafrechtsnovellen vor dem Völkerbund, in: DJ 1936, S. $213 \mathrm{ff}$.

Groscurth, H.: Tagebücher eines Abwehroffiziers 1938-1940, hrsg. von H. Krausnick, H. C. Deutsch, Stuttgart 1970.

Gruchmann, L.: „Blutschutzgesetz“ und Justiz, in: Aus Politik und Zeitgeschichte, Beilage zur Wochenzeitung „Das Parlament“ Nr. 48 vom 30. 11. 1985, S. 35 ff., u. VfZ 1983, S. 418 ff.

Gruchmann, L.: Die Entstehung des Testamentsgesetzes vom 31. Juli 1938: Nationalsozialistische „Rechtserneuerung“ und Reformkontinuität, in: ZfNRG 1985, S. $53 \mathrm{ff}$.

Gruchmann, L.: Erlebnisbericht Werner Pünders über die Ermordung Klauseners am 30. Juni 1934 und ihre Folgen, in: VfZ 1971, S. $404 \mathrm{ff}$.

Gruchmann, L.: Euthanasie und Justiz im Dritten Reich, in: VfZ 1972, S. $235 \mathrm{ff}$.

Gruchmann, L.: Hitler und die Justiz. Das Tischgespräch vom 20. August 1942, in: VfZ 1964, S. $86 \mathrm{ff}$.

Gruchmann, L.: „Nacht- und Nebel“-Justiz. Die Mitwirkung deutscher Strafgerichte an der Bekämpfung des Widerstandes in den besetzten westeuropäischen Ländern 1942-1944, in: VfZ 1981, S. $342 \mathrm{ff}$.

Gruchmann, L.: Rechtssystem und nationalsozialistische Justizpolitik, in: Broszat, M., H. Möller (Hrsg.): Das Dritte Reich, München 1983, S. 83 ff.

Gruchmann, L.: Die „Reichsregierung“ im Führerstaat. Stellung und Funktion des Kabinetts im nationalsozialistischen Herrschaftssystem, in: Doeker, G., W. Steffani (Hrsg.): Klassenjustiz und Pluralismus, Hamburg 1973, S. $187 \mathrm{ff}$.

Gruchmann, L.: Ein unbequemer Amtsrichter im Dritten Reich. Aus den Personalakten des Dr. Lothar Kreyßig, in: VfZ 1984, S. $463 \mathrm{ff}$.

Gründel, H. [u. a.] (Hrsg.): Die Strafprozeßordnung für das Deutsche Reich vom 22.3.1924 nebst Gerichtsverfassungsgesetz, 19. Aufl., Berlin 1933-36.

Gürtner, F.: Einheitsstaat oder Bundesstaat? Zur Rechtsfrage, in: Dem bayerischen Volke, München 1930 , S. $400 \mathrm{ff}$.

Gürtner, F.: Der Gedanke der Gerechtigkeit in der deutschen Strafrechtserneuerung, in: DJ 1935 , S. $1241 \mathrm{ff}$.

Gürtner, F.: An die jungen Juristen! Rede des Reichsministers, gehalten im Gemeinschaftslager „Hanns Kerrl“ am 19. Juni 1934, in: DJ 1934, S. 786 f.

Gürtner, F. (Hrsg.): Das kommende deutsche Strafrecht. Allgemeiner Teil. Bericht über die Arbeit der amtlichen Strafrechtskommission. 1.Aufl., Berlin 1934; 2. Aufl., Berlin 1935.

Gürtner, F. (Hrsg.): Das kommende deutsche Strafrecht. Besonderer Teil. Bericht über die Arbeit der amtlichen Strafrechtskommission. 1.Aufl., Berlin 1935; 2. Aufl., Berlin 1936. 
Gürtner, F. (Hrsg.): Das kommende deutsche Strafverfahren. Bericht der amtlichen Strafprozeßkommission, Berlin 1938.

Gürtner, F.: Macht und Recht, in: Coburger Zeitung Nr. 111 vom 14. 5. 1929.

Gürtner, F., R. Freisler: Das neue Strafrecht. Grundsätzliche Gedanken zum Geleit. 2.Aufl., Berlin 1936.

Gürtner, F.: Richter und Rechtsanwalt im neuen Staat, in: Jb. d. AkDR 1933/34, S. 155 ff.

Gürtner, F.: Auf dem Wege zur einheitlichen Justiz. Rede des Reichsjustizministers Dr. Gürtner bei der Übernahme der bayerischen Justizverwaltung, in: DJ 1935, S. $81 \mathrm{f}$.

Gürtner, F. (Hrsg.): 200 [Zweihundert] Jahre Dienst am Recht. Gedenkschrift aus Anlaß des 200jährigen Gründungstages des Preußischen Justizministeriums, Berlin 1938.

Gürtner, F[ritz]: Das Gesetz über Maßnahmen der Staatsnotwehr vom 3. Juli 1934, in: JZ 1954, S. $763 \mathrm{f}$.

Güstrow, D.: Tödlicher Alltag - Strafverteidiger im Dritten Reich, Berlin 1981.

Gumbel, E. J.: Verräter verfallen der Feme, Berlin 1929.

Gumbel, E. J.: Vier Jahre politischer Mord (Nachdr. d. Ausg. v. 1922), Heidelberg 1980.

Gutachten des Instituts für Zeitgeschichte, Bd. 1., München 1958, Bd. 2., Stuttgart 1966.

Haacke, W. von: Drei Jahre Zentralstaatsanwaltschaft, in: DJ 1936, S. 1257 f.

Hachenburg, M.: Juristische Rundschau, in: DJZ 1924, S. $366 \mathrm{ff}$.

Hackel, F. (Hrsg.): Von der Jugendherberge Hohnstein zum „Schutzhaftlager Hohnstein“, Berlin, Potsdam 1949.

Haidn, C., F. Fischer: Das Recht der NSDAP, München 1936.

Handbuch der Justizverwaltung, bearb. im Büro des Reichsjustizministeriums, Berlin 1942.

Hanisch, E.: Politische Prozesse vor dem Sondergericht im Reichsgau Salzburg 1939-1945, in: Weinzierl, E., K. R. Stadler (Hrsg.): Justiz und Zeitgeschichte, Wien 1977, S. $210 \mathrm{ff}$.

Hannover, H., E. Hannover: Politische Justiz 1918-1933, Frankfurt a. M. 1966, 2.Aufl., 1977.

Hartung, F.: Jurist unter vier Reichen, Köln, Berlin, Bonn, München 1971.

Hattenhauer, H.: Das NS-Volksgesetzbuch, in: Buschmann, W. [u.a.] (Hrsg.): Festschrift für Rudolf Gmür zum 70. Geburtstag, 28. Juli 1983, Bielefeld 1983, S. $255 \mathrm{ff}$.

Hattenhauer, H.: Vom Reichsjustizamt zum Bundesministerium der Justiz. Stellung und Einfluß der obersten deutschen Justizbehörde in ihrer 100jährigen Geschichte, in: Vom Reichsjustizamt zum Bundesministerium der Justiz. Festschrift zum 100jährigen Gründungstag des Reichsjustizamtes am 1. Januar 1877, Köln 1977, S. 9 ff.

Hedemann, J. W., H. Lehmann, W. Siebert (Hrsg.): Volksgesetzbuch. Grundregeln und Buch I, Entwurf und Erläuterungen, München, Berlin 1942.

Hedemann, J. W.: Das Volksgesetzbuch der Deutschen, München u. Berlin 1941.

Hegler, A.: Die Eidesreform, Stuttgart 1930.

Heiber, H.: Der Fall Grünspan, in: VfZ 1957, S. $134 \mathrm{ff}$.

Heilbrunn, O.: Rechtspflegerassessoren, in: DRiZ 1933, S. 11 f.

Heinrichs, E.: Diebstahl und Unterschlagung nach der Denkschrift des Preußischen Justizministers über nationalsozialistisches Strafrecht, in: DJ 1934, S. $158 \mathrm{ff}$.

Heintzler, W.: Der rote Faden, Stuttgart 1983.

Hempfer, W.: Die nationalsozialistische Staatsauffassung in der Rechtsprechung des Preußischen Oberverwaltungsgerichts, Berlin 1974.

Henkel, H.: Das Deutsche Strafverfahren, Hamburg 1943.

Henkel, H.: Die Hauptverhandlung im kommenden Strafverfahren, in: DStR 1935, S. $401 \mathrm{ff}$.

Heuber, W.: Der Bund Nationalsozialistischer Deutscher Juristen und die Deutsche Rechtsfront, in: Nationalsozialistisches Handbuch für Recht und Gesetzgebung, München 1935, S. $1566 \mathrm{ff}$.

Hildebrandt: Grundsätzliches zum Dienst im Gemeinschaftslager Hanns Kerrl, in: DJ 1936, S. $1757 \mathrm{ff}$.

Hillermeier, H. (Hrsg.): Im Namen des deutschen Volkes. Todesurteile des Volksgerichtshofes, Darmstadt, Neuwied 1980.

Hillmayr, H.: Roter und Weißer Terror in Bayern nach 1918, München 1974.

Himmler, H.: Aufgaben und Aufbau der Polizei des Dritten Reiches, in: Pfundtner, H. (Hrsg.):

Dr. Wilhelm Frick und sein Ministerium, München 1937, S. $125 \mathrm{ff}$.

Hippel, F. von: Die nationalsozialistische Herrschaftsordnung als Warnung und Lehre. Eine juristische Betrachtung, 2. Aufl., Tübingen 1947. 
Hippel, F. von: Die Perversion von Rechtsordnungen, Tübingen 1955.

Hirsch, M., D. Majer, J. Meinck (Hrsg.): Recht, Verwaltung und Justiz im Nationalsozialismus, Köln 1984.

Hirschberg, M., F. Thimme (Hrsg.): Der Fall Fechenbach. Juristische Gutachten, Tübingen 1924.

Hirschberg, M.: Das Fehlurteil im Strafprozeß. Zur Pathologie der Rechtsprechung, Stuttgart 1960.

Hitler, A.: Mein Kampf, 25.-27. Aufl., München 1933.

Der Hitler-Ludendorff-Prozeß vor dem Münchner Volksgericht, in: Breuer, R. (Hrsg.): Politische Prozesse. Aktenmäßige Darstellung, H. 4, Berlin 1924.

Hockerts, H. G.: Die Sittlichkeitsprozesse gegen katholische Ordensangehörige und Priester 1936/37. Eine Studie zur nationalsozialistischen Herrschaftstechnik und zum Kirchenkampf, Mainz 1971.

Hodenberg, H. Frhr. v.: Der Aufbau der Rechtspflege nach der Niederlage von 1945, in: 250 Jahre Oberlandesgericht Celle 1711-1961, Celle 1961, S. $121 \mathrm{ff}$.

Hoegner, W.: Die bayerische Justiz vor dem Untersuchungsausschuß, in: Die Justiz, Bd. 3., $1927 / 28$, S. $315 \mathrm{ff}$.

Hoegner, W.: Hitler und Kahr. Die bayerischen Napoleonsgrößen von 1923. Ein im Untersuchungsausschuß des Bayerischen Landtags aufgedeckter Justizskandal, 2. Teil, München 1928.

Hoegner, W.: Der schwierige Außenseiter. Erinnerungen eines Abgeordneten, Emigranten und Ministerpräsidenten, München 1959.

Hoegner, W.: Die Verratene Republik. Deutsche Geschichte 1919-1933, München 1979.

Höss, R.: Kommandant in Auschwitz. Autobiographische Aufzeichnungen, eingel. und komment. von M. Broszat, Stuttgart 1958.

Hofer, W. [u. a.] (Hrsg.): Der Reichstagsbrand. Eine wissenschaftliche Dokumentation, Bd. 1., Berlin 1972; Bd. 2., München 1978.

Hofmann, H. H.: Der Hitlerputsch. Krisenjahre deutscher Geschichte 1920-1924, München 1961.

Holzhauer, H.: Die Scheidungsgründe in der nationalsozialistischen Familienrechtsgesetzgebung, in: NS-Recht in historischer Perspektive, hrsg. vom Institut für Zeitgeschichte, München, Wien 1981.

Honolka, B.: Die Kreuzelschreiber. Ärzte ohne Gewissen. Euthanasie im Dritten Reich, Hamburg 1961.

Horkenbach, C.: Das Deutsche Reich von 1918 bis heute, Berlin 1935.

Horn, N. [u. a.] (Hrsg.): Europäisches Rechtsdenken in Geschichte und Gegenwart. Festschrift für Helmut Coing zum 70. Geburtstag, München 1982.

Horstkotte, H.: Die Anfänge der Strafrechtsreform - aktuelle und überholte Fragen, in: Vom Reichsjustizamt zum Bundesministerium der Justiz, hrsg. vom Bundesministerium der Justiz, Köln 1977, S. $325 \mathrm{ff}$.

Hubatsch, W.: Hindenburg und der Staat, Göttingen 1966.

Huber, E. R.: Deutsche Verfassungsgeschichte seit 1789, Bd. 7.: Ausbau, Schutz und Untergang der Weimarer Republik, Stuttgart, Berlin, Köln, Mainz 1984.

Huber, E. R.: Verfassungsrecht des Großdeutschen Reiches, Hamburg 1939.

Hubernagel, G.: Nationalsozialistische Rechtsauffassung und Generalklauseln, in: Nationalsozialistisches Handbuch für Recht und Gesetzgebung, München 1935, S. $971 \mathrm{ff}$.

Huck, J.: Reichsminister Paul Frhr. von Eltz-Rübenach. Sein Leben und Wirken 1875-1943, in: Unser Porz. Beiträge zur Geschichte von Amt und Stadt Porz, hrsg. vom Heimatverein Porz e.V. in Verb. mit dem Stadtarchiv Porz, H. 2, Porz a.Rh. 1961, S. $31 \mathrm{ff}$.

Hüttenberger, P.: Die Gauleiter. Studie zum Wandel des Machtgefüges in der NSDAP, Stuttgart 1969.

Hüttenberger, P.: Heimtückefälle vor dem Sondergericht München 1933-1939, in: Bayern in der NS-Zeit, Bd. 4., München 1981, S. 435 ff.

Ibach, K.: Kemma. Wuppertaler Lager der SA 1933, Wuppertal 1948, Nachdruck Wuppertal 1981.

Jahrbuch des Deutschen Rechtsstandes, hrsg. vom Bund Nat.-Soz. Deutscher Juristen, Berlin 1936.

Janowitz, M.: Soziale Lage und Mobilität in Westdeutschland, in: Kölner Zeitschrift für Soziologie und Sozialpsychologie 1958, S. $1 \mathrm{ff}$. 
Jasper, G.: Justiz und Politik in der Weimarer Republik, in: VfZ 1982, S. $167 \mathrm{ff}$.

Jasper, G.: Der Schutz der Republik. Studien zur staatlichen Sicherung der Demokratie in der Weimarer Republik 1922-1930, Tübingen 1963.

Jochmann, W. (Hrsg.): Adolf Hitler. Monologe im Führerhauptquartier 1941-1944. Die Aufzeichnungen Heinrich Heims, München 1982.

Johe, W.: Die gleichgeschaltete Justiz. Organisation des Rechtswesens und Politisierung der Rechtsprechung 1933-1945, dargestellt am Beispiel des Oberlandesgerichtsbezirks Hamburg, Frankfurt a. M. 1967.

Jonas, M.: Die Justizausbildungsordnung vom 22. Juli 1934, in: DJ 1934, S. $995 \mathrm{ff}$.

Justiz und Nationalsozialismus, hrsg. von der Niedersächsischen Landeszentrale für politische Bildung, Hannover 1985.

Kalle: Die Parteigerichtsbarkeit und der Beamte, in: DJ 1938, S. $1555 \mathrm{ff}$.

Kaul, F. K.: Geschichte des Reichsgerichts, Bd. 4., 1933-1945, Glashütten/Taunus 1971.

Kelz: Zur Anrechnung von Schutzhaft auf die Strafe, in: DJ 1936, S. $1609 \mathrm{ff}$.

Kempner, R. M.: Blueprint of the Nazi Underground, Research Studies of the State College of Washington 1945.

Kern, E.: Geschichte des Gerichtsverfassungsrechts, München, Berlin 1954.

Kern, E.: Der gesetzliche Richter, Berlin 1927.

Kern, E.: Das neue Gerichtsverfassungs- und Strafprozeßrecht, Freiburg 1934.

Kerrl, H.: Die Bedeutung des Gemeinschaftslagers der Referendare in Preußen, in: DJ 1934, S. $237 \mathrm{ff}$.

Kerrl, H.: Das Dritte Reich, in: Gürtner, F. (Hrsg.): 200 Jahre Dienst am Recht. Gedenkschrift aus Anlaß des 200jährigen Gründungstages des Preußischen Justizministeriums, Berlin 1938, S. $167 \mathrm{ff}$.

Kershaw, I.: Der Hitler-Mythos. Volksmeinung und Propaganda im Dritten Reich, Stuttgart 1980.

Keßler: Die Bedeutung der Kriegsgesetzgebung für die künftige Rechtsgestaltung, in: DR, Ausg. B, 1941, S. $96 \mathrm{f}$.

Kewer, L.: Aus der Geschichte des Oberlandesgerichts Hamm, in: Rechtspflege zwischen Rhein und Weser, Festschrift zum 150jährigen Bestehen des Oberlandesgerichts Hamm, Hamm 1970 , S. $37 \mathrm{ff}$.

Kielmansegg, J. A. Graf: Der Fritschprozeß 1938. Ablauf und Hintergründe, Hamburg 1949.

Kimmel, G.: Das Konzentrationslager Dachau, in: Bayern in der NS-Zeit, Bd. 2., München 1979, S. $349 \mathrm{ff}$.

Kitzinger, F.: Der Fall Fechenbach, in: ZStrWiss. 1923, S. $136 \mathrm{ff}$.

Klatt: Die Unterbringung der alten Kämpfer in Beamtenstellen, in: DDJB 1938, S. $837 \mathrm{f}$.

Klee: Strafe ohne geschriebenes Gesetz, in: DJZ 1934, Sp. $639 \mathrm{ff}$.

Klee, E. (Hrsg.): Dokumente zur „Euthanasie“, Frankfurt a. M. 1985.

Klee, E.: „Euthanasie“ im NS-Staat. Die „Vernichtung lebensunwerten Lebens“, Frankfurt a. M. 1983.

Klein, A.: Hundert Jahre Akten - hundert Jahre Fakten, in: Klein, A., G. Rennen (Hrsg.): Justitia Coloniensis. Landgericht und Amtsgericht Köln erzählen ihre Geschichte(n), Köln 1981, S. $89 \mathrm{ff}$.

Klein, A., G. Rennen (Hrsg.): Justitia Coloniensis. Landgericht und Amtsgericht Köln erzählen ihre Geschichte(n), Köln 1981.

Klein, A.: Die rheinische Justiz und der rechtsstaatliche Gedanke in Deutschland - Zur Geschichte des Oberlandesgerichtsbezirks Köln und der Gerichtsbarkeit in seinem Bezirk, in: Wolffram, J., A. Klein (Hrsg.): Recht und Rechtspflege in den Rheinlanden, Köln 1969.

Klein, F.: Zur Vorbereitung der faschistischen Diktatur durch die deutsche Großbourgeoisie 1929-1932, in: ZfGWiss. (Ost-Berlin) 1953, S. $872 \mathrm{ff}$.

Klenner, J.: Verhältnis von Partei und Staat 1933-1945. Dargestellt am Beispiel Bayerns, München 1974.

Kluke, P.: Der Fall Potempa, in: VfZ 1957, S. $279 \mathrm{ff}$.

Koch, W.-P.: Die Reform des Strafverfahrensrechts im Dritten Reich unter besonderer Berücksichtigung des StVO-Entwurfs 1939, jur. Diss. Erlangen 1972.

König, S.: Vom Dienst am Recht. Rechtsanwälte als Strafverteidiger im Nationalsozialismus, Berlin 1987. 
Kogon, E.: Der SS-Staat. Das System der deutschen Konzentrationslager, München 1974.

Kohlrausch, E.: Strafprozeßordnung und Gerichtsverfassungsgesetz, Berlin 1936.

Kolbe, D.: Reichsgerichtspräsident Dr. Erwin Bumke. Studien zum Niedergang des Reichsgerichts und der deutschen Rechtspflege, Karlsruhe 1975.

Kosthorst, E., B. Walters: Konzentrations- und Strafgefangenenlager im Dritten Reich: Beispiel Emsland, 3 Bde., Düsseldorf 1983.

Kramer, G. F.: The Influence of National-Socialism on the Courts of Justice and the Police, in: The Third Reich, hrsg. vom International Council for Philosophy and Humanistic Studies, London 1955, S. $595 \mathrm{ff}$.

Kramer, H. (Hrsg.): Braunschweig unterm Hakenkreuz, Braunschweig 1981.

Kramer, H.: Oberlandesgerichtspräsidenten und Generalstaatsanwälte als Gehilfen der NS-„Euthanasie“. Selbstentlastung der Justiz für die Teilnahme am Anstaltsmord, in: KJ 1984, S. 25 ff.

Krausnick, H.: Der 30. Juni 1934. Bedeutung, Hintergründe, Verlauf, in: Aus Politik und Zeitgeschichte, Beilage zur Wochenzeitschrift „Das Parlament“ Nr. 25 vom 30.6. 1954, S. $317 \mathrm{ff}$.

Krausnick, H.: Vorgeschichte und Beginn des militärischen Widerstandes gegen Hitler, in: Vollmacht des Gewissens, hrsg. von der Europäischen Publikation e.V., Bd. 1., München 1956, S. $175 \mathrm{ff}$.

Krug, K., K. Schäfer, F. W. Stolzenburg: Strafrechtliche Verwaltungsvorschriften, 1.Aufl., Berlin 1936; 2. Aufl., Berlin 1939; 3.Aufl., Berlin 1943.

Kübler, F. K.: Der deutsche Richter und das demokratische Gesetz, in: Archiv für die civilistische Praxis 1963, S. $104 \mathrm{ff}$.

Kuhn, R.: Die Vertrauenskrise der Justiz (1926-1928). Der Kampf um die „Republikanisierung“ der Rechtspflege in der Weimarer Republik, Köln 1983.

Lammers, H. H., H. Pfundtner (Hrsg.): Die Verwaltungsakademie. Ein Handbuch für den Beamten im nationalsozialistischen Staat, Berlin 1935.

Lang, A. R. (Hrsg.): Festschrift für Karl Bengl, München 1984.

Lange, H.: Die Entwicklung der Wissenschaft vom Bürgerlichen Recht, Tübingen 1941.

Langhoff, W.: Die Moorsoldaten, 4.Aufl., Stuttgart 1978.

Latour, C.: Goebbels' „Außerordentliche Rundfunkmaßnahmen“ 1939-1942, in: VfZ 1963, S. $418 \mathrm{ff}$.

Lauer, K.: Die polizeirechtliche Bedeutung der Verordnung zum Schutz von Volk und Staat, in: RVerwBl. 1935, S. $168 \mathrm{ff}$.

Lehmann, R.: Der Eid im Strafprozeß, in: DJ 1933, S. $872 \mathrm{ff}$.

Lehmann, R.: Das Ermessen des Strafrichters beim Absehen von der Vereidigung, in: DJ 1936, S. $1008 \mathrm{ff}$.

Lehmann, R.: Die Strafprozeßnovelle vom 28. Juni 1935, in: DJ 1935, S. $999 \mathrm{ff}$.

Lein, A.: Braunschweiger Justiz im Nationalsozialismus: zwischen Anpassung und ,innerer Emigration“, in: Kramer, H. (Hrsg.): Braunschweig unterm Hakenkreuz, Braunschweig 1981, S. $61 \mathrm{ff}$.

Lewy, G.: Die katholische Kirche und das Dritte Reich, München 1965.

Lösener, B., F. Knost: Die Nürnberger Gesetze über das Reichsbürgerrecht und den Schutz des deutschen Blutes und der deutschen Ehre nebst Durchführungsverordnungen, Berlin 1936.

Löwe, E., A. Hellweg, W. Rosenberg: Die Strafprozeßordnung für das Dritte Reich vom 22.3. 1924 nebst Gerichtsverfassungsgesetz, Berlin 1934.

Lorenzen, S.: Die Juden und die Justiz. Bearbeitet im Auftrage des Reichsministers der Justiz, 2.Aufl., Berlin, Hamburg 1943.

Majer, D.: „Fremdvölkische“ im Dritten Reich. Ein Beitrag zur nationalsozialistischen Rechtssetzung und Rechtspraxis in Verwaltung und Justiz unter besonderer Berücksichtigung der eingegliederten Ostgebiete und des Generalgouvernements, Boppard a. Rh. 1981.

Majer, D.: Justiz und NS-Staat. Zum Einfluß der NSDAP auf die Organisation und Personalpolitik der Justiz 1933-1945, in: DRiZ 1978, S. 47 ff.

Majer, D.: Zum Verhältnis von Staatsanwaltschaft und Polizei im Nationalsozialismus, in: Reifner, U., B.-R. Sonnen (Hrsg.): Strafjustiz und Polizei im Dritten Reich, Frankfurt a. M., New York 1984, S. $121 \mathrm{ff}$.

Marxen, K.: Der Kampf gegen das liberale Strafrecht. Eine Studie zum Antiliberalismus in der Strafrechtswissenschaft der zwanziger und dreißiger Jahre, Berlin 1975. 
Matthias, E., R. Morsey (Hrsg.): Das Ende der Parteien 1933, Düsseldorf 1960.

Mau, H.: Die „Zweite Revolution“ - 30. Juni 1934, in: VfZ 1953, S. $119 \mathrm{ff}$.

Mayer, H.: Das Analogieverbot im gegenwärtigen deutschen Strafrecht, in: SJZ 1947, Sp. $12 \mathrm{f}$.

Medicus, F. A.: Reichsverwaltung und Landesverwaltung, in: Die Verwaltungsakademie, Bd. 2., Beitrag 23, Berlin 0. J.

Meier-Scherling: Die Benachteiligung der Juristin zwischen 1933 und 1945, in: DRiZ 1975, S. $10 \mathrm{f}$.

Meinck, J.: Justiz und Justizfunktion im Dritten Reich, in: ZfNRG 3 (1981), S. 28 ff.

Meissner, O.: Staatssekretär unter Ebert, Hindenburg, Hitler, Hamburg 1950.

Messerschmidt, M., F. Wüllner: Die Wehrmachtjustiz im Dienste des Nationalsozialismus. Zerstörung einer Legende, Baden-Baden 1987.

Mettgenberg, W. (Hrsg.): Erwin Bumke zum 65.Geburtstag, Berlin 1939.

Meyer, G.: Nacht über Hamburg. Berichte und Dokumente 1933-1945, Frankfurt a. M. 1971.

Mezger, E.: Willensstrafrecht, Gefährdungsstrafrecht und Erfolgsstrafrecht, in: DJZ 1934, Sp. $97 \mathrm{ff}$.

Mitscherlich, A., F. Mielke: Medizin ohne Menschlichkeit. Dokumente des Nürnberger Ärzteprozesses, Frankfurt a. M., Hamburg 1960.

Mlynek, K.: Die „Reichskristallnacht“, in: „Reichskristallnacht“ in Hannover, Hannover 1978, S. $56 \mathrm{ff}$.

Mößmer, F.: Rassenmischehe und geltendes Recht, in: ZAkDR 1934, S. $86 \mathrm{ff}$.

Mommsen, H.: Aufgabenkreis und Verantwortlichkeit des Staatssekretärs der Reichskanzlei Dr. Wilhelm Kritzinger, in: Gutachten des IfZ, Bd. 2., Stuttgart 1966, S. 369 ff.

Mommsen, H.: Beamtentum im Dritten Reich, Stuttgart 1966.

Mommsen, H.: Der Reichstagsbrand und seine politischen Folgen, in: VfZ 1964, S. $351 \mathrm{ff}$.

Moritz, K., E. Noam: NS-Verbrechen vor Gericht, 1945-1955. Dokumente aus hessischen Justizakten, Wiesbaden 1978.

Morsey, R.: Die Deutsche Zentrumspartei, in: Matthias, E., R. Morsey (Hrsg.): Das Ende der Parteien 1933, Düsseldorf 1960, S. $281 \mathrm{ff}$.

Morsey, R.: Hitlers Verhandlungen mit der Zentrumsführung am 31. Januar 1933, in: VfZ 1961, S. $182 \mathrm{ff}$.

Morsey, R.: Politische Gesinnungsprüfung der Oberlandesgerichtspräsidenten in Preußen 1933. Ein Beitrag zur Gleichschaltung der Justiz zu Beginn des „Dritten Reiches“, in: Blümel, W., D. Merten, H. Quaritsch (Hrsg.): Verwaltung im Rechtsstaat. Festschrift für Carl Hermann Ule zum 80. Geburtstag am 26. Februar 1987, Köln, Berlin, Bonn, München 1987, S. $209 \mathrm{ff}$.

Morsey, R.: Rezension von E. Reitter, Franz Gürtner - Politische Biographie eines deutschen Juristen 1881-1941, Berlin 1976, in: JAB 1977, S. $84 \mathrm{f}$.

Müller, I.: Furchtbare Juristen. Die unbewältigte Vergangenheit unserer Justiz, München 1987.

Müller, I.: Das Strafprozeßrecht des Dritten Reiches, in: Reifner, U., B.-R. Sonnen (Hrsg.): Strafjustiz und Polizei im Dritten Reich, Frankfurt a. M., New York 1984, S. $59 \mathrm{ff}$.

Müller, J.: Bis zur letzten Konsequenz. Ein Leben für Frieden und Freiheit, München 1975.

Müller, K. A. von: Aus Gärten der Vergangenheit. Erinnerungen, Bd. 1., 1882-1914, Stuttgart 1951.

Müller, K. A. von: Im Wandel einer Welt. Erinnerungen, Bd. 3., 1919-1932, München 1966.

Müller, K.-J.: Das Heer unter Hitler. Armee und nationalsozialistisches Regime 1933-1940, Stuttgart 1969.

Münch, I. von (Hrsg.): Gesetze des NS-Staates. Textsammlung, 2. Aufl., Bad Homburg 1982.

Nadler, M.: Die Arbeit der Personalabteilung des Reichsjustizministeriums im Jahre 1935, in: DJ 1936, S. 3 f.

Nationalsozialistisches Strafrecht. Denkschrift des Preußischen Justizministers, Berlin 1933.

Naucke, W.: Die Aufhebung des strafrechtlichen Analogieverbots 1935, in: NS-Recht in historischer Perspektive, hrsg. vom Institut für Zeitgeschichte, München, Wien 1981, S. $71 \mathrm{ff}$.

Naucke, W.: Die Mißachtung des strafrechtlichen Rückwirkungsverbotes 1933-1945, in: Horn, N. [u.a.] (Hrsg.): Europäisches Rechtsdenken in Geschichte und Gegenwart. Festschrift für Helmut Coing zum 70. Geburtstag, München 1982, S. $225 \mathrm{ff}$.

Neeße, F.: Führergewalt. Die Entwicklung und Gestaltung hoheitlicher Gewalt im Deutschen Reiche, Tübingen 1940. 
Neeße, G.: Das Gesetz zur Sicherung der Einheit von Partei und Staat, Dresden 1934.

Neufeldt, H.-J., J. Huck, G. Tessin: Zur Geschichte der Ordnungspolizei 1936-1945 (als Manuskript gedruckt), Koblenz 1957.

Neugebauer, W.: Politische Justiz in Österreich 1934-1945, in: Weinzierl, E., K. R. Stadler (Hrsg.): Justiz und Zeitgeschichte, Wien 1977, S. 169 ff.

Neuhäusler, J.: Kreuz und Hakenkreuz. Der Kampf des Nationalsozialismus gegen die katholische Kirche und der kirchliche Widerstand, T. 2., München 1946.

Neumann, F.: Behemoth. Struktur und Praxis des Nationalsozialismus 1933-1944, Köln, Frankfurt a. M. 1977.

Neuordnung des Strafverfahrensrechts. Denkschrift des NS-Rechtswahrerbundes zum Entwurf einer Strafverfahrensordnung, einer Friedensrichter- und Schiedsmannsordnung und eines Gerichtsverfassungsgesetzes der amtlichen Strafprozeßkommission des Reichsjustizministeriums, Berlin 1937.

Niederreuther: Gedanken zur Reform des Strafverfahrens, in: DJ 1936, S. $770 \mathrm{ff}$.

Niemöller, W.: Macht gegen Recht. Der Prozeß Martin Niemöller, München 1952.

Niethammer, E.: Das Reichsgericht als Schrittmacher der Entwicklung des Strafverfahrens nach geltendem Recht und in Zukunft, in: DStR 1937, S. $125 \mathrm{ff}$.

Nipperdey, H. C. (Hrsg.): Das deutsche Privatrecht in der Mitte des 20.Jahrhunderts. Festschrift für Heinrich Lehmann zum 80. Geburtstag, Berlin, Frankfurt a. M., Tübingen 1956.

Noack, E.: Formalismus im Strafprozeß, in: DR 1934, S. $357 \mathrm{f}$.

Noam, E., W.-A. Kropat: Juden vor Gericht 1933-1945. Dokumente aus hessischen Justizakten, Wiesbaden 1975.

NS-Recht in historischer Perspektive, hrsg. vom Institut für Zeitgeschichte, München, Wien 1981.

Oetker, F.: Zur Reform der Strafprozeßordnung, in: ZAkDR 1935, S. 670 ff., 1936, S. 215 ff., S. 292 ff., S. 590 ff., S. 625 ff., S. $1010 \mathrm{ff}$.

Oldenhage, K.: Die Pfalz und das Saarland während des Krieges (1940-1945). Aus den Lageberichten des Oberlandesgerichtspräsidenten und Generalstaatsanwaltes in Zweibrücken, in: Jb. f. westdeutsche Landesgeschichte 5 (1979), S. $303 \mathrm{ff}$.

Ostendorf, H., H. ter Veen: Das „Nürnberger Juristenurteil“. Eine kommentierte Dokumentation, Frankfurt a. M., New York 1985.

Ostler, F.: Die deutschen Rechtsanwälte 1871-1971, 2.Aufl., Essen 1982.

Ostler, F.: Rechtsanwälte in der NS-Zeit - Fakten und Erinnerungen, in: Anwaltsblatt 1983, S. $50 \mathrm{ff}$.

Palandt, O.: Die Arbeit der Ausbildungsabteilung (RJP) im Jahre 1937, in: DJ 1938, S. 22 ff.

Palandt, O., H. Richter: Die Justizausbildungsordnung des Reiches, Berlin 1939.

Papen, F. von: Der Wahrheit eine Gasse, München 1952.

Partei-Statistik, Stand 1. Januar 1935, Bd. 1., hrsg. vom Reichsleiter der NSDAP, Berlin 1935.

Perk, W.: Die Hölle im Moor, Frankfurt a. M. 1970.

Personalverzeichnis des höheren Justizdienstes, bearb. im Büro des Reichsjustizministeriums, Berlin 1938.

Peters: Die falsche Aussage und ihre Bekämpfung, in: DStR 1935, S. $145 \mathrm{ff}$.

Peters: Zur Neuordnung des Strafverfahrens, in: ZStr.Wiss. 1937, S. $40 \mathrm{f}$.

Petzina, D.: Autarkiepolitik im Dritten Reich. Der nationalsozialistische Vierjahresplan, Stuttgart 1968.

Pfundtner, H. (Hrsg.): Dr. Wilhelm Frick und sein Ministerium, München 1937.

Pfundtner, H., R. Neubert unter Mitw. von F. A. Medicus (Hrsg.): Das neue Reichsrecht. Ergänzbare Sammlung des geltenden Rechts seit dem Ermächtigungsgesetz mit Erläuterungen, Berlin $1933 \mathrm{ff}$.

Pichinot, H.-R.: Die Akademie für Deutsches Recht. Aufbau und Entwicklung einer öffentlichrechtlichen Körperschaft des Dritten Reiches, jur. Diss. Kiel 1981.

Picker, H.: Hitlers Tischgespräche im Führerhauptquartier 1941-42, Bonn 1951.

Platen-Hallermund, A.: Die Tötung Geisteskranker in Deutschland, Frankfurt a. M. 1948.

Plischke, K.: Kommt das Gesetz gegen Rasseverrat? in: Das Schwarze Korps vom 10. 4. 35, S. 10.

Ploetz: Das Dritte Reich. Ursprünge, Ereignisse, Wirkungen. Hrsg. von M. Broszat u. N. Frei in Verb. mit dem Institut für Zeitgeschichte, München, Würzburg 1983. 
Plum, G.: Staatspolizei und innere Verwaltung 1934-1936, in: VfZ 1965, S. $191 \mathrm{ff}$.

Popp, H.: Die nationalsozialistische Sicht einiger Institute des Zivilprozeß- und Gerichtsverfassungsrechts. Dargestellt am Beispiel des Gesetzes über die Mitwirkung des Staatsanwalts in Bürgerlichen Rechtssachen vom 15.7.1941 (RGBI. I S. 383), Frankfurt a. M., Bern, New York 1986.

Popplow, U.: Der Novemberpogrom 1938 in Münden und Göttingen, in: Göttinger Jb. 1980, S. $177 \mathrm{ff}$.

Portmann, H.: Dokumente um den Bischof von Münster, Münster 1948.

Präg, W., W. Jacobmeyer (Hrsg.): Das Diensttagebuch des deutschen Generalgouverneurs in Polen 1939-1945, Stuttgart 1975.

Preuß, B.: Punishment by analogy in national socialist penal law, in: The Journal of criminal law and criminology 1936, S. $847 \mathrm{ff}$.

Preußen contra Reich vor dem Staatsgerichtshof. Stenogrammbericht der Verhandlungen vor dem Staatsgerichtshof in Leipzig vom 10. bis 14. und vom 17. Oktober 1932, Berlin 1933.

Der Prozeß gegen die Hauptkriegsverbrecher vor dem Internat. Militärgerichtshof (Trial of the Major War Criminals before the Internat. Military Tribunal). Nürnberg, 14. Nov. $1945-1$. Okt. 1946, Nürnberg, Internat. Militärgerichtshof 1947-49, Bd. 11., 20., 26., 27., 28., 31., 32., 33., 36., 42.

Pünder, H.: Politik in der Reichskanzlei. Aufzeichnungen aus den Jahren 1929-1932, hrsg. von T. Vogelsang, Stuttgart 1961.

Püschel, W.: Der Niedergang des Rechts im Dritten Reich, Reutlingen 1947.

Radbruch, G.: Entwurf eines Allgemeinen Deutschen Strafgesetzbuches 1922, hrsg. von T. Dehler, Tübingen 1952.

Radbruch, G.: Des Reichsjustizministeriums Ruhm und Ende, in: SJZ 1948, Sp. $58 \mathrm{ff}$.

Rasehorn, Theo: Der Republikanische Richterbund, in: Recht und Politik 1983, S. $17 \mathrm{ff}$.

Rechtspflege zwischen Rhein und Weser. Festschrift zum 150jährigen Bestehen des Oberlandesgerichts Hamm, hrsg. vom Verein für Rechtsgeschichte im Gebiet des Oberlandesgerichts Hamm e.V., Hamm 1970.

Rechtssicherheit und Gerechtigkeit, Deutscher Juristentag 1936, hrsg. vom NSRB, Berlin o. J.

Reichshandbuch der deutschen Gesellschaft. Das Handbuch der Persönlichkeiten in Wort und Bild. Hrsg.: Deutscher Wirtschaftsverlag, Bd. 1.2., Berlin 1930-31.

Reichsjustizminister Dr. Gürtner in Sachsen und Thüringen. Kundgebungen in Dresden, Weimar und Jena, in: DJ 1935, S. $249 \mathrm{ff}$.

„Reichskristallnacht“ in Hannover. Eine Ausstellung zur 40. Wiederkehr des 9. November 1938, hrsg. vom Historischen Museum am Hohen Ufer, Hannover 1978.

Reifner, U.: Juristen im Nationalsozialismus, in: ZRP 1983, S. 13 ff.

Reifner, U. (Hrsg.): Das Recht des Unrechtsstaates. Arbeitsrecht und Staatsrechtswissenschaft im Faschismus, Frankfurt a. M. 1982.

Reifner, U., B.-R. Sonnen (Hrsg.): Strafjustiz und Polizei im Dritten Reich, Frankfurt a. M., New York 1984 .

Reitter, E.: Franz Gürtner. Politische Biographie eines deutschen Juristen 1881-1941, Berlin 1976.

Rempe, H.: Verfahren gegen Flüchtige und gegen Abwesende, die sich der Wehrpflicht entzogen haben, in: JW 1935, S. $2334 \mathrm{ff}$.

Revermann, K.: Die stufenweise Durchbrechung des Verfassungssystems der Weimarer Republik in den Jahren 1930 bis 1933. Eine staatsrechtliche und historisch-politische Analyse, Münster 1959.

Richter, H.: Gemeinschaftsleiter im Hanns-Kerrl-Lager, in: DJ 1936, S. $1761 \mathrm{f}$.

Richter, W.: Die Richter der Oberlandesgerichte der Bundesrepublik. Eine berufs- und sozialstatistische Analyse, in: Hamburger Jahrbuch für Wirtschafts- und Gesellschaftspolitik 1960, S. $41 \mathrm{ff}$.

Rietzsch: Die Abwehr des Gewohnheitsverbrechertums. Deutsche Gesetze und Gesetzentwürfe bis zur Machtübernahme, in: DJ 1938, S. $134 \mathrm{ff}$.

Rilk, O.: Zum neuen Strafverfahrensrecht, in: JW 1937, S. $2333 \mathrm{ff}$.

Robinsohn, H.: Justiz als politische Verfolgung. Die Rechtsprechung in „Rassenschandefällen“ beim Landgericht Hamburg 1936-1943, Stuttgart 1977. 
Roloff, E.-A.: Bürgertum und Nationalsozialismus 1930-1933. Braunschweigs Weg ins Dritte Reich, Hannover 1961.

Rosenberg, A.: Der Mythus des 20.Jahrhunderts. Eine Wertung der seelisch-geistigen Gestaltenkämpfe unserer Zeit, 4.Aufl., München 1932.

Rosenberg, A. (Hrsg.): Das Parteiprogramm. Wesen, Grundsätze und Ziele der NSDAP, 21.Aufl., München 1941.

Rothenberger, C. (Hrsg.): Das Hanseatische Oberlandesgericht. Gedenkschrift zu seinem 60jährigen Bestehen, Hamburg 1939.

Rothenberger, C.: Die Stellung des Richters im Führerstaat, in: DR 1939, S. $831 \mathrm{ff}$.

Rottleuthner, H. (Hrsg.): Recht, Rechtsphilosophie und Nationalsozialismus. Vorträge aus der Tagung der Deutschen Sektion der Internationalen Vereinigung für Rechts- und Sozialphilosophie in der BRD vom 11. und 12. Oktober 1982 in Berlin, Wiesbaden 1983.

Rüping, H.: Auflockerung im Strafverfahrensrecht, in: Rottleuthner, H. (Hrsg.): Recht, Rechtsphilosophie und Nationalsozialismus, Wiesbaden 1983, S. $65 \mathrm{ff}$.

Rüping, H.: Bibliographie zum Strafrecht im Nationalsozialismus, München 1985.

Rüping, H.: Nationalsozialistische Rechtsprechung am Beispiel der SS- und Polizei-Gerichte, in: NZfStR 1983, S. $112 \mathrm{ff}$.

Rüping, H.: Nullum crimen sine poena. Zur Diskussion um das Analogieverbot im Nationalsozialismus, in: Herzberg, R. D. (Hrsg.): Festschrift für Dietrich Oehler zum 70.Geburtstag, Köln, Berlin, Bonn, München 1985, S. $27 \mathrm{ff}$.

Rüping, H.: Strafjustiz im Führerstaat, in: Goltdammer's Archiv für Strafrecht 1984, S. $297 \mathrm{ff}$.

Rüping, H.: Strafrechtspflege und politische Justiz im Umbruch vom liberalen Rechtsstaat zum NS-Regime, in: Becker, J. (Hrsg.): 1933. Fünfzig Jahre danach. Die nationalsozialistische Machtergreifung in historischer Perspektive, München 1983, S. $153 \mathrm{ff}$.

Rüping, H.: Streng, aber gerecht. Schutz der Staatssicherheit durch den Volksgerichtshof, in: JZ 1984, S. $815 \mathrm{ff}$.

Rüthers, B.: Die unbegrenzte Auslegung. Zum Wandel der Privatrechtsordnung im Nationalsozialismus, Tübingen 1968.

Rumschöttel, H.: Das Bayerische Staatsministerium der Justiz 1799-1966, in: Lang, A. R. (Hrsg.): Festschrift für Karl Bengl, München 1984, S. $329 \mathrm{ff}$.

Sadila-Mantau, H. H. (Hrsg.): Unsere Reichsregierung, Berlin 1936.

Salje, P. (Hrsg.): Recht und Unrecht im Nationalsozialismus, Münster 1985.

Sauer, F.: Das Reichsjustizministerium, Berlin 1939.

Schäfer, E.: Die Auflockerung des Verfahrens im künftigen Strafprozeß und der Gedanke der materiellen Gerechtigkeit, in: DStR 1935, S. $247 \mathrm{ff}$.

Schäfer, E.: Die leitenden Gedanken der beiden Gesetze zur Änderung des Strafgesetzbuches sowie zur Anderung des Strafverfahrens und des Gerichtsverfassungsgesetzes vom 28. Juni 1935, in: DJ 1935, S. $991 \mathrm{ff}$.

Schäfer, L.: Die Einzelheiten der Strafgesetznovelle vom 28. Juni 1935, in: DJ 1935, S. $994 \mathrm{ff}$.

Schäfer, L., O. Wagner, J. Schafheutle: Gesetz gegen gefährliche Gewohnheitsverbrecher und über Maßregeln der Sicherung und Besserung mit dem dazugehörigen Ausführungsgesetz, Berlin 1934.

Schäfer, L.: Die Strafgesetz- und Strafprozeßnovelle vom 24. April 1934, in: DJZ 1934, S. 632 ff.

Schaffstein, F.: Revision und Berufung im künftigen Strafverfahren, in: DStR 1935, S. $465 \mathrm{ff}$.

Scheffler, W.: Judenverfolgung im Dritten Reich, Berlin 1960.

Schicksale jüdischer Juristen in Hamburg im Dritten Reich, Hrsg.: Verein für Hamburgische Geschichte, Hamburg 1985.

Schimmler, B.: Recht ohne Gerechtigkeit. Zur Tätigkeit der Berliner Sondergerichte im Nationalsozialismus, Berlin 1984.

Schlegelberger, F.: Abschied vom BGB. Vortrag, gehalten in der Universität zu Heidelberg am 25. Januar 1937, Berlin 1937.

Schlegelberger, F.: Der Weg ins Reich, in: Gürtner, F. (Hrsg.): 200 Jahre Dienst am Recht, Berlin 1938, S. $173 \mathrm{ff}$.

Schmid, H.: Erinnerungen aus den Jahren 1930 bis 1945, in: 250 Jahre Oberlandesgericht Celle 1711-1961, Celle 1961, S. $106 \mathrm{ff}$.

Schmid, R.: Erinnerungen an den NS-Rechtswahrerbund, in: JZ 1954, S. $263 \mathrm{f}$. 
Schmidt, E.: Einführung in die Geschichte der deutschen Strafrechtspflege, Göttingen 1965.

Schmidt, J.: Martin Niemöller im Kirchenkampf, Hamburg 1971.

Schmitt, C.: Der Führer schützt das Recht. Zur Reichstagsrede Adolf Hitlers vom 13. Juli 1934, in: DJZ 1934, Sp. $945 \mathrm{ff}$.

Schmitt, C.: Das Problem der Legalität, in: Schmitt, C.: Verfassungsrechtliche Aufsätze aus den Jahren 1924-1954. Materialien zu einer Verfassungslehre, Berlin 1958, S. $440 \mathrm{ff}$.

Schmitt, C.: Der Weg des deutschen Juristen, in: DJZ 1934, Sp. $691 \mathrm{ff}$.

Schneller, O.: Der Aufbau der Reichsjustizverwaltung im Geschäftsbereich der Haushaltsabteilung, in: DJ 1936, S. $11 \mathrm{ff}$.

Schöllkopf, A.: Die Versuche einer Reform der Strafgerichtsverfassung in den Jahren 1919-1923, jur. Diss. Tübingen 1937.

Schönke, A.: Einige Bemerkungen über die Sicherungsverwahrung im Ausland, in: DJ 1938, S. $142 \mathrm{ff}$.

Schönke, A.: Die Frage der richterlichen Rechtsschöpfung (Analogie) im neueren ausländischen Schrifttum, in: DJ 1936, S. 1466 ff., 1937, S. 1613 ff., 1939, S. 207 ff.

Schoetensack, A., W. Töwe (Hrsg.): Grundfragen des neuen Strafverfahrensrechts. Denkschrift des Ausschusses für Strafprozeßrecht der Strafrechtsabteilung der Akademie für Deutsches Recht, Stuttgart 1937.

Schorn, H.: Die Gesetzgebung des Nationalsozialismus als Mittel der Machtpolitik, Frankfurt a. M. 1963.

Schorn, H.: Der Richter im Dritten Reich. Geschichte und Dokumente, Frankfurt a.M. 1959.

Schütz, H.: Bamberger Berichte. Über Stimmung und Haltung der Bevölkerung des Oberlandesgerichtsbezirks Bamberg während des 2. Weltkrieges, Bamberg 1983.

Schütz, H.: Justiz im „Dritten Reich“. Dokumentation aus dem Bezirk des Oberlandesgerichts Bamberg, Bamberg 1984.

Schumacher, U.: Staatsanwaltschaft und Gericht im Dritten Reich. Zur Veränderung der Kompetenzverteilung im Strafverfahren unter Berücksichtigung der Entwicklung in der Weimarer Republik und in der Bundesrepublik, jur. Diss. Köln 1985.

Schulz, B.: Der Republikanische Richterbund, Frankfurt a. M. 1982.

Schulz, G.: Die Anfänge des totalitären Maßnahmenstaates, in: Bracher K. D., W. Sauer, G. Schulz: Die nationalsozialistische Machtergreifung. T. 2., durchges. u. erg. Ausg., Frankfurt a. M., Berlin, Wien 1974.

Schumacher, O.: 14 Tage „Kurgast“ im Hanns-Kerrl-Gemeinschaftslager, in: DJ 1938, S. $1081 \mathrm{f}$.

Schwarz, O.: Bausteine zum Strafprozeß im nationalsozialistischen Staat, in: Der Gerichtssaal 1935, S. $361 \mathrm{f}$.

Schwarz, O.: Das Reichsgericht als Tatsacheninstanz, in: ZAkDR 1940, S. $139 \mathrm{ff}$.

Das Schwarzbuch. Tatsachen und Dokumente. Die Lage der Juden in Deutschland 1933, hrsg. vom Comité des Delegations Juives, Paris 1934.

Schweling, O. P.: Die deutsche Militärjustiz in der Zeit des Nationalsozialismus, Marburg 1977.

Schwend, K.: Bayern zwischen Monarchie und Diktatur. Beiträge zur Bayerischen Frage in der Zeit von 1918 bis 1933, München 1954.

Schwerin von Krosigk, L. Graf: Es geschah in Deutschland. Menschenbilder unseres Jahrhunderts, Tübingen, Stuttgart 1951.

Schwinge, E., W. Siebert: Das neue Untreuestrafrecht in strafrechtlicher Beleuchtung, Berlin 1933.

Seifert, K.-H.: Das Nachwuchsproblem des Staatsdienstes, in: Deutsche Verwaltung 1944, S. $121 \mathrm{ff}$.

Sellert, W.: Nationalsozialistische Ideologie und der Versuch zu einer Reform des Strafprozeßrechts im Dritten Reich, in: Justiz und Nationalsozialismus, Hrsg.: Niedersächsische Landeszentrale für politische Bildung, Hannover 1985, S. $59 \mathrm{ff}$.

Siegert, K.: Richter, Staatsanwalt und Beschuldigter im Strafprozeß des neuen Staates, in: ZStrWiss. 1934/35, Bd. 54, S. 24.

Simon, D.: Waren die NS-Richter „unabhängige Richter“ im Sinne des $§ 1$ GVG? in: Rechtshistorisches Journal 1985, S. $102 \mathrm{ff}$.

Sommer, K.: Verreichlichung der Justiz, in: Leipziger Zeitschrift für Deutsches Recht 1928, S. $1159 \mathrm{ff}$. 
Sommer, W.: Partei und Staat, in: DJZ 1936, Sp. $593 \mathrm{ff}$.

Spieler, C.: Preußischer Geist im Gemeinschaftslager in Jüterbog, in: DJ 1933, S. $641 \mathrm{ff}$.

Spohr, W.: Das Recht der Schutzhaft, in: DJ 1934, S. $58 \mathrm{ff}$.

Spohr, W.: Recht der Schutzhaft, Berlin 1937.

Spruth, B.: Diebstahl und Unterschlagung im neuen Strafrecht, in: JW 1934, S. 144.

Staff, I.: Justiz im Dritten Reich. Eine Dokumentation, Frankfurt a. M. 1964, 2.Aufl., 1978.

Staud, E.: Die bürgerliche Rechtspflege in Kriegszeiten, in: DJ 1939, S. $1482 \mathrm{ff}$.

Steger, B.: Der Hitlerprozeß und Bayerns Verhältnis zum Reich, in: VfZ 1977, S. $441 \mathrm{ff}$.

Steininger, P. A., K. Leszcyński (Hrsg.): Fall 3. Das Urteil im Juristenprozeß, gefällt am 4. Dezember 1947 vom Militärgerichtshof III der Vereinigten Staaten von Amerika, Berlin (Ost) 1969.

Steinlechner, W.: Der Richter im Dritten Reich. Status - Unabhängigkeit - Persönlichkeit, jur. Diss. Mainz 1974.

Stern, H. N.: Political Crime and Justice in the Weimarer Republic, The Johns Hopkins University, phil. Diss. Baltimore 1966.

Stock, U.: Der Fritsch-Prozeß 1938. Seine rechtliche Beurteilung und seine Lehren, in: Nipperdey, H. C. (Hrsg.): Das deutsche Privatrecht in der Mitte des 20.Jahrhunderts. Festschrift für Heinrich Lehmann zum 80. Geburtstag, Berlin, Frankfurt a. M., Tübingen 1956, S. $625 \mathrm{ff}$.

Stock, U.: Zur Strafprozeßerneuerung, Leipzig 1935.

Stolleis, M.: Gemeinwohlformeln im nationalsozialistischen Recht, Berlin 1974.

Stolleis, M.: Nationalsozialistisches Recht, in: Erler, A., E. Kaufmann (Hrsg.): Handwörterbuch zur deutschen Rechtsgeschichte, Bd. 2., Berlin 1978, S. 882 ff.

Stolleis, M.: Die Rechtsordnung des Nationalsozialismus, in: Jurist. Schulung 1982, S. 645 ff.

Stolleis, M., D. Simon: Vorurteile und Werturteile der rechtshistorischen Forschung zum Nationalsozialismus, in: NS-Recht in historischer Perspektive, hrsg. vom Institut für Zeitgeschichte, München, Wien 1981, S. $13 \mathrm{ff}$.

Stolzenburg: Die Entlassung aus der Sicherungsverwahrung, in: DJ 1938, S. $217 \mathrm{ff}$.

Stolzenburg: Die vorläufigen Ergebnisse der letzten Amnestie in Preußen, in: DJ 1934, S. $1210 \mathrm{f}$.

Strafgesetzbuch. Strafprozeßordnung. Gerichtsverfassungsgesetz, nebst den wichtigsten Nebengesetzen, hrsg. vom Reichsjustizministerium, Berlin 1935.

Die Strafrechtsnovellen vom 28. Juni 1935, Amtliche Sonderveröffentlichungen der DJ, Nr. 10, Berlin 1939.

Strasser, O.: Hitler und ich, Konstanz 1948.

Strauss, W.: Das Reichsministerium des Innern und die Judengesetzgebung. Aufzeichnungen von Dr. Bernhard Lösener, in: VfZ 1961, S. 262 ff.

Stritzke, O.: Streik und Aussperrung im neuen Strafrecht, in: DJ 1933, S. 754.

Stuckart, W., H. Globke: Kommentare zur deutschen Rassengesetzgebung, München, Berlin 1936.

Studien zur Geschichte der Konzentrationslager, Schriftenreihe der VfZ, Nr. 21, Stuttgart 1970.

Suchomel, H.: Die außerordentliche Nichtigkeitsbeschwerde im künftigen Strafverfahren, in: Mettgenberg, W. (Hrsg.): Erwin Bumke zum 65.Geburtstag, Berlin 1939, S. $135 \mathrm{ff}$.

Tag des Deutschen Rechts 1939. 6. Reichstagung des Nationalsozialistischen Rechtswahrerbundes, hrsg. vom Nationalsozialistischen Rechtswahrerbund, Berlin, Leipzig, Wien 1939.

Tegtmeyer, W.: Der außerordentliche Einspruch, in: DR 1939, S. $2057 \mathrm{ff}$.

Terhorst, K.-L.: Polizeiliche planmäßige Überwachung und polizeiliche Vorbeugungshaft im Dritten Reich. Ein Beitrag zur Rechtsgeschichte vorbeugender Verbrechensbekämpfung, Heidelberg 1985, zugl. jur. Diss., Bonn 1984.

Thévoz, R., H. Branig, C. Lowenthal-Hensel: Pommern 1934/35 im Spiegel von Gestapo-Lageberichten und Sachakten, Bd. 1. u. 2., Köln, Berlin 1974.

Thierack, G.: Die Aufgaben der Beauftragten des Reichsministers der Justiz, in: DJ 1935, S. 3 f.

Thierack, G.: Grundzüge eines nationalsozialistischen Strafprozesses unter besonderer Berücksichtigung des Ergebnisses der Arbeit des Ausschusses für Strafprozeßrecht der Akademie für Deutsches Recht, in: ZAkDR 1935, S. $94 \mathrm{ff}$.

Thiesing: Drei Monate als Beauftragter des Reichsministers der Justiz für die Abteilung Württemberg-Baden, in: DJ 1935, S. $704 \mathrm{ff}$. 
Thimme, F.: Zum Münchener Prozeß, in: Vorwärts Nr. 514 vom 31. 10. 1922.

Thoss, P.: Das subjektive Recht in der gliedschaftlichen Bindung. Zum Verhältnis von Nationalsozialismus und Privatrecht, Frankfurt a. M. 1968.

Thul, E. J.: Das Landgericht Koblenz im nationalsozialistischen Unrechtsstaat, in: 150 Jahre Landgericht Koblenz, Boppard a. Rh. 1970, S. 72 ff.

Timpke, H. (Hrsg.): Dokumente zur Gleichschaltung des Landes Hamburg, Frankfurt a. M. 1964.

Timpke, H.: Das KL Fuhlsbüttel, in: Studien zur Geschichte der Konzentrationslager, Stuttgart 1970, S. $11 \mathrm{ff}$.

Tobias, F.: Der Reichstagsbrand. Legende und Wirklichkeit, Rastatt 1962.

Die Toten von Dachau. Deutsche und Österreicher, hrsg. vom Staatskommissariat für rassisch, religiös und politisch Verfolgte in Bayern, München 1947.

Trumpp, T.: Franz von Papen, der preußisch-deutsche Dualismus und die NSDAP in Preußen. Ein Beitrag zur Vorgeschichte des 20. Juli 1932, phil. Diss. Tübingen 1963.

Tschirschky, F. G. von: Erinnerungen eines Hochverräters, Stuttgart 1972.

Tuchel, J., R. Schattenfroh: Zentrale des Terrors. Prinz-Albrecht-Straße 8. Das Hauptquartier der Gestapo, Berlin 1987.

Tutas, H. E.: Nationalsozialismus und Exil. Die Politik des Dritten Reiches gegenüber der deutschen politischen Emigration 1933-1939, München, Wien 1975.

Der Unrechtsstaat. Recht und Justiz im Nationalsozialismus, hrsg. von der Redaktion Kritische Justiz, Bd. 1., Frankfurt a. M. 1979, Bd. 2., Baden-Baden 1984.

Ursachen und Folgen. Vom deutschen Zusammenbruch 1918 und 1945 bis zur staatlichen Neuordnung Deutschlands in der Gegenwart, hrsg. von H. Michaelis, E. Schraepler, Bd. 1-23, Berlin 1958-76.

Verfügungen, Anordnungen, Bekanntgaben, hrsg. von der Partei-Kanzlei, Bd. 1. und 2., München 1942.

Vogel, H. J., H. Simon, A. Podlech (Hrsg.): Die Freiheit des Anderen. Festschrift für Martin Hirsch, Baden-Baden 1981.

Vogelsang, Th.: Neue Dokumente zur Geschichte der Reichswehr, in: VfZ 1954, S. $397 \mathrm{ff}$.

Vogelsang, Th.: Zur Politik Schleichers gegenüber der NSDAP 1932, in: VfZ 1958, S. $86 \mathrm{ff}$.

Vogelsang, Th.: Reichswehr, Staat und NSDAP. Beiträge zur deutschen Geschichte 1930-1932, Stuttgart 1962.

Volkmann, K.: Recht und Rechtspflege im Nationalsozialismus, in: Rottenburger Jb. f. Kirchengesch. 1983, Bd. 2., S. $59 \mathrm{ff}$.

Vollmacht des Gewissens, hrsg. von der Europäischen Publikation e.V., München 1956, 3. Aufl., Frankfurt a. M., Berlin 1960.

Vollmer, B.: Volksopposition im Polizeistaat. Gestapo- und Regierungsberichte 1934-1936, Stuttgart 1957.

Vom Reichsjustizamt zum Bundesministerium der Justiz. Festschrift zum 100jährigen Gründungstag des Reichsjustizamtes am 1. Januar 1877, hrsg. vom Bundesministerium der Justiz, Köln 1977.

Wachinger, M.: Rechtsschöpferische Rechtsprechung des Reichsgerichts auf materiell-strafrechtlichem Gebiet, in: Mettgenberg, W. (Hrsg.): Erwin Bumke zum 65. Geburtstag, Berlin 1939, S. $78 \mathrm{f}$.

Waechter: $\S 4$ der Volksschädlingsverordnung und Antragsdelikte, in: DJ 1940, S. $473 \mathrm{ff}$.

Wagner, A.: Der Richter. Geschichte. Aktuelle Fragen. Reformprobleme, Karlsruhe 1959.

Wagner, A.: Die Umgestaltung der Gerichtsverfassung und des Verfahrens- und Richterrechts im nationalsozialistischen Staat, in: Weinkauff, H., A. Wagner: Die deutsche Justiz und der Nationalsozialismus, Stuttgart 1968, S. $191 \mathrm{ff}$.

Wagner, W.: Der Volksgerichtshof im nationalsozialistischen Staat. Die deutsche Justiz und der Nationalsozialismus, T. 3., Stuttgart 1974.

Walk, J. (Hrsg.): Das Sonderrecht für die Juden im NS-Staat. Eine Sammlung der gesetzlichen Maßnahmen und Richtlinien - Inhalt und Bedeutung, Heidelberg, Karlsruhe 1981.

Wassermann, R.: Kontinuität oder Wandel. Konsequenz aus der NS-Herrschaft für die Entwicklung der Justiz nach 1945, Hannover 1984.

Watt, D. C.: Die bayerischen Bemühungen um die Ausweisung Hitlers 1924, in: VfZ 1958, S. $270 \mathrm{ff}$. 
Weinkauff, H.: Die deutsche Justiz und der Nationalsozialismus. Wagner, A.: Die Umgestaltung der Gerichtsverfassung und des Verfahrens- und Richterrechts im nationalsozialistischen Staat. Die deutsche Justiz und der Nationalsozialismus, T. 1., Stuttgart 1968.

Weinzierl, E., K. R. Stadler (Hrsg.): Justiz und Zeitgeschichte, Wien 1977.

Weiß, W.: Der Volksgerichtshof des Deutschen Reiches, in: DR, S. $518 \mathrm{f}$.

Wieacker, F.: Privatrechtsgeschichte der Neuzeit. Unter besonderer Berücksichtigung der deutschen Entwicklung, 2.Aufl., Göttingen 1967.

Wogatzky, H.: 120 Jahre oberste Hanseatische Gerichte, in: Rothenberger, C. (Hrsg.): Das Hanseatische Oberlandesgericht, Hamburg 1939, S. $15 \mathrm{ff}$.

Wolf, G. J. (Hrsg.): Dem bayerischen Volke, München 1930.

Wolff, P.: Ohne Maske, Hamburg 1948.

Wolffram, J., A. Klein (Hrsg.): Recht und Rechtspflege in den Rheinlanden, Köln 1969.

Wrobel, H.: Die Anfechtung der Rassenmischehe. Diskriminierung und Entrechtung der Juden 1933-1945, in: Der Unrechtsstaat. Recht und Justiz im Nationalsozialismus, hrsg. von der Redaktion Kritische Justiz, Bd. 2., Baden-Baden 1984, S. 99 ff.

Wrobel, H.: Der deutsche Richterbund im Jahre 1933. Skizzen eines Ablaufs, in: KJ 1982, S. 323 ff., u. DRiZ 5/1983, S. 157 ff.

Wrobel, H.: Otto Palandt zum Gedächtnis. 1.5.1877-3.12.1951, in: KJ 1982, S. $1 \mathrm{ff}$.

Zeiler, A.: Münzfernsprechbetrug? in: JW 1935, S. $476 \mathrm{ff}$.

275 [Zweihundertfünfundsiebzig] Jahre Oberappellationsgericht - Oberlandesgericht Celle, hrsg. vom Präsidenten des Oberlandesgerichts Celle, Celle 1986.

250 [Zweihundertfünfzig] Jahre Oberlandesgericht Celle 1711-1961, Celle 1961.

\section{Seit der Erstauflage 1988 veröffentlichte Quellen und Literatur}

Akten der Reichskanzlei. Weimarer Republik, hrsg. für die Historische Kommission bei der Bayerischen Akademie der Wissenschaften von Hans Dietrich Erdmann, für das Bundesarchiv von Hans Booms. Das Kabinett von Papen. Band 1: Juni bis September 1932. Band 2: September bis Dezember 1932, bearb. von Karl-Heinz Minuth. - Boppard a. Rhein, 1989.

Akten der Reichskanzlei. Regierung Hitler 1933-1945, hrsg. für die Historische Kommission bei der Bayerischen Akademie der Wissenschaften von Hans Günther Hockerts, für das Bundesarchiv von Friedrich P. Kahlenberg. Band II 1934/35. Teilband 1: August 1934 - Mai 1935. Teilband 2: Juni - Dezember 1935, bearb. von Friedrich Hartmannsgruber. - München 1999.

Albin, Silke: Richterlicher Widerstand in totalitären Systemen. Darstellung am Beispiel der Justiz im Nationalsozialismus und in der DDR. - In: Recht u. Politik 34 (1998), S. 40-55.

Anders-Baudisch, Freia: Nationalsozialistische Sondergerichte im "Reichsgau Sudetenland“. Ein Beitrag zur Erforschung der nationalsozialistischen Justizpraxis in Böhmen und Mähren (Magisterarbeit). - Bielefeld 1997.

Angermund, Ralph: Deutsche Richterschaft 1919-1945. Krisenerfahrung, Illusion, politische Rechtsprechung. - Frankfurt a. M. 1990.

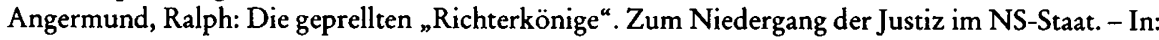
Hans Mommsen und Susanne Willems (Hrsg.): Herrschaftsalltag im Dritten Reich. - Düsseldorf 1988, S. 304-373.

Angermund, Ralph: Justiz als Instrument politischer Verfolgung. Rechtsprechung im Rheinland und in Westfalen 1933-1945. - In: Anselm Faust (Hrsg.): Verfolgung und Widerstand im Rheinland und Westfalen 1933-1945. - Köln 1992, S. 50-64.

Bästlein, Klaus: Als Recht zu Unrecht wurde. Zur Entwicklung der Strafjustiz im Nationalsozialismus. - In: Aus Politik und Zeitgeschichte Nr. 13 vom 24. März 1989, S. 3-18.

Bästlein, Klaus: Funktion und Struktur der Justiz im NS-Staat und der DDR. - In: Juristische Zeitgeschichte 2 (1994), S. 39-51. 
Bästlein, Klaus: Sondergerichte in Norddeutschland als Verfolgungsinstanz. - In: Norddeutschland im Nationalsozialismus, hrsg. von Frank Bajohr, Hamburg 1993, S. 218-238.

Bästlein, Klaus: Vom hanseatischen Richtertum zum nationalsozialistischen Justizverbrechen. Zur Person und Tätigkeit Curt Rothenbergers 1896-1959. - In: Justizbehörde Hamburg (Hrsg.): „Für Führer, Volk und Vaterland ...". Hamburger Justiz im Nationalsozialismus. - Hamburg 1992, S. 74-145.

Bästlein, Klaus: Zur „Rechts“-Praxis des Schleswig-Holsteinischen Sondergerichts 1937-1945. - In: Heribert Ostendorf (Hrsg.): Strafverfolgung und Strafverzicht. Festschrift zum 125jährigen Bestehen der Staatsanwaltschaft Schleswig-Holstein. - Köln [u.a.] 1992, S. 93-185.

Ball, Wolfgang: „Panzertruppe der Rechtspflege“. Die Tätigkeit der Sondergerichte in der Pfalz während der Herrschaft des Nationalsozialismus. - In: Gerhard Nestler und Hannes Ziegler (Hrsg.): Die Pfalz unterm Hakenkreuz. Eine deutsche Provinz während der nationalsozialistischen Terrorherrschaft. - Landau 1993, S. 141-160.

Ball, Wolfgang: Sondergerichte im Oberlandesgerichtsbezirk Zweibrücken. In: Sven Paulsen (Hrsg.): 175 Jahre pfälzisches Oberlandesgericht. - Neustadt an der Weinstraße 1990, S. 227-255.

Bartels, Gundolf: Zivilrechtsprechung in Oldenbourg 1933-1945. Dargestellt vor allem am Beispiel des Ehe- und Familienrechts. - In: 175 Jahre Oberlandesgericht Oldenbourg. Festschrift, Köln [u. a.] 1989, S. 253-288.

Bennhold, Martin (Hrsg.): Spuren des Unrechts. Recht und Nationalsozialismus. Beiträge zur historischen Kontinuität. - Köln 1989.

Benz, Wolfgang, Hermann Graml und Hermann Weiß (Hrsg.): Enzyklopädie des Nationalsozialismus. - München 1998.

Benz, Wolfgang: Jüdische Juristen unter dem nationalsozialistischen Regime: von der Entrechtung zur Verfolgung und Vernichtung. - In: Juristische Zeitgeschichte 8 (1999), S. 19-36.

Bies, Luitwin: Neue Dokumente zum Berliner Katholiken-Prozeß 1937. In memoriam Dr. Dr. h.c. Joseph Cornelius Rossaint. - In: Beiträge zur Geschichte der Arbeiterbewegung 34 (1992), S. 83-94.

Blasius, Dirk: „Bürgerlicher Tod“. Der NS-Unrechtsstaat und die deutschen Juden. - In: Geschichte in Wissenschaft und Unterricht 41 (1990), S. 129-144.

Blasius, Dirk: Ehescheidung in Deutschland im 19. und 20. Jahrhundert. - Frankfurt a. M. 1992.

Blumberg-Ebel, Anna: Sondergerichtsbarkeit und „politischer Katholizismus“im Dritten Reich. Mainz 1990.

Börner, Fritjof: Die Bedeutung der Generalklauseln für die Umgestaltung der Rechtsordnung in der nationalsozialistischen Zeit. - Frankfurt a. M. [u. a.] 1989

Bohn, Robert und Uwe Danker (Hrsg.): „Standgericht der inneren Front“: das Sondergericht Altona/Kiel 1932-1945. - Hamburg 1998.

Brandenburgisches Oberlandesgericht (Hrsg.): Unrecht beim Namen genannt. Gedenken an Lothar Kreissig am 30. Oktober 1998. - Baden-Baden 1998.

Browder, George C.: Foundations of the Nazi police state. The formation of Sipo and SD. - Lexington 1990.

Brüggemeier, Gert: Oberstes Gesetz ist das Wohl des Volkes. Das Projekt des „Volksgesetzbuches". - In: Juristenzeitung 1990, S. 24-28.

Bundesminister der Justz (Hrsg.): Im Namen des Deutschen Volkes. Justiz und Nationalsozialismus. Katalog zur Ausstellung des Bundesministers der Justiz. - Köln 1989.

Cheng, Yar-Che: Die Ausnahme bestimmte die Regel: das antirechtsstaatliche Strafrecht des Nationalsozialismus. - Frankfurt a.M. (u. a.) 1996.

Chowaniec; Elisabeth: Der „Fall [Hans von] Dohnanyi“ 1943-1945. Widerstand, Militärjustiz, SSWillkür. - München 1991.

Christoph, Jürgen: Die politischen Reichsamnestien 1918-1933. - Frankfurt a. M. 1988.

Colmorgen, Eckhard und Klaus-Detlev Godau-Schüttke, Frauen vor Gericht. Die ,Rechtsprechung' des Schleswig-Holsteinischen Sondergerichts wegen „verbotenen Umgangs mit Kriegsgefangenen" (1940-1945), in: Schleswig-Holsteinische Anzeigen. Justizministerialblatt, hrsg. vom Justizminister des Landes Schleswig-Holstein, Teil A, Nr. 6 (Juni) 1995, S. 145-154.

Dachs, Johann: Tod durch das Fallbeil: der deutsche Scharfrichter Johann Reichhart (1893-1972). Regensburg 1996.

Davy, Ulrike, H. Fuchs, Herbert Hofmeister, Judit Marte und Ilse Reiter (Hrsg.): Nationalsozialis- 
mus und Recht. Rechtssetzung und Rechtswissenschaft in Österreich unter der Herrschaft des Nationalsozialismus. - Wien 1990.

Debus, Karl Heinz: Christen und Juden und die Justiz im Dritten Reich im Oberlandesgerichtsbezirk Zweibrücken. - In: Sven Paulsen (Hrsg.): 175 Jahre pfälzisches Oberlandesgericht. - Neustadt an der Weinstraße 1990, S. 181-205.

Diederichsen, Uwe: Nationalsozialistische Ideologie in der Rechtsprechung des Reichsgerichts zum Ehe- und Familienrecht. - In: Ralf Dreier und Wolfgang Sellert (Hrsg.): Recht und Justiz im "Dritten Reich“. - Frankfurt a. M. 1989, S. 241-272.

Dießelhorst, Malte: Die Euthanasie im „Dritten Reich“. - In: Ralf Dreier und Wolfgang Sellert (Hrsg.): Recht und Justiz im „Dritten Reich“. - Frankfurt a. M. 1989, S. 118-135.

Diestelkamp, Bernhard und Michael Stolleis (Hrsg.): Justizalltag im Dritten Reich. - Frankfurt a. M. 1988.

Diewald-Kerkmann, Gisela, Kerstin Kunz und Andreas Knobelsdorf: Vor braunen Richtern. Die Verfolgung von Widerstandshandlungen, Resistenz und sogenannter Heimtücke in Bielefeld 1933-45, hrsg. von Stadtarchiv und Landesgeschichtlicher Bibliothek Bielefeld. - Bielefeld 1992.

Dörner, Bernward: „Heimtücke“: das Gesetz als Waffe: Kontrolle, Abschreckung und Verfolgung in Deutschland 1933-1945. - Paderborn [u. a.] 1998.

Dörner, Bernward: Justiz und Judenmord. Zur Unterdrückung von Äußerungen über den Genozid an den europäischen Juden durch die deutsche Justiz. - In: Jb. für Antisemitismusforschung 4 (1995), S. 226-253.

Dörner, Christine: Bestrafung Frühreifer“ und Erziehung „Unreifer“. Die Geschichte des Strafunmündigkeitsalters in den drei deutschen Jugendgerichtsgesetzen von 1923, 1943 und 1953. - In: Z. für Neuere Rechtsgeschichte 16 (1994), S. 58-84.

Dörner, Christine: Erziehung durch Strafe. Die Geschichte des Jugendstrafvollzugs 1871-1945, Weinheim/München 1991.

Douma, Eva: Deutsche Anwälte zwischen Demokratie und Diktatur: 1930-1955. - Frankfurt/M. 1998.

Douma, Eva: Rechtsanwälte als Staatsdiener. Der „Einsatz“ der Rechtsanwälte in der Justiz während des Zweiten Weltkrieges. - In: Juristische Zeitgeschichte 1 (1993), S. 103-130.

Dreier, Ralf und Wolfgang Sellert (Hrsg.): Recht und Justiz im „Dritten Reich“. - Frankfurt a.M. 1989.

Drobisch, Klaus: Alltag im Zuchthaus Luckau 1933 bis 1939. - In: Dietrich Eichholtz (Hrsg.): Brandenburg in der NS-Zeit. Studien und Dokumente. - Berlin 1993, S. 247-272.

Drobisch, Klaus: Konzentrationslager und Justizhaft. Versuch einer Zusammenschau. - In: Helge Grabitz (Hrsg.), Die Normalität des Verbrechens. - Berlin 1994, S. 280-297.

Düwell, Franz-Josef und Thomas Vormbaum (Hrsg.): Themen juristischer Zeitgeschichte (1) Schwerpunktthema: Recht und Nationalsozialismus. - Baden-Baden 1998.

Ecke, Felix: Die Nürnberger Gesetze. Ein Kapitel "Recht“ im Unrechtsstaat. - In: Heinz Mohnhaupt (Hrsg.), Die Rechtsgeschichte in beiden deutschen Staaten 1988-1990. Beispiele, Parallelen, Positionen. - Frankfurt a. M. 1991, S. 586-603.

Eckert, Joachim [u.] Antonia Tens: Hitler und die Juristen. Äußerungen und tatsächliche Politik. In: Recht u. Politik 29 (1993), S. 34-50.

Eckler, Irene: Die Vormundschaftsakte 1935-1958: Verfolgung einer Familie wegen „Rassenschan-

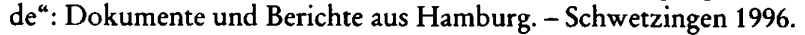

Eder-Stein, Irmtraut: Plünderung im Freimachungsgebiet 1939/40. Ein Straftatbestand in Strafrecht und Rechtsprechung des NS-Staates. - In: Wolfgang Haubrichs, Wolfgang Laufer und Reinhard Schneider (Hrsg.): Zwischen Saar und Mosel. Festschrift für Hans-Walter Herrmann. - Saarbrücken 1995, S. 419-435.

Eiber, Ludwig: Polizei, Justiz und Verfolgung in München 1933 bis 1945. - In: München - Hauptstadt der Bewegung, hrsg. vom Stadtmuseum München, München 1993, S. 235-243.

Endemann, Fritz: Nationalsozialistische Strafjustiz in Stuttgart. - In: Schwäbische Heimat 42 (1991), S. 303-313.

Engelmann, Bernt: Die unsichtbare Tradition. Band 2: Rechtsverfall, Justizterror und das schwere Erbe: Ein Beitrag zur Geschichte der deutschen Strafjustiz 1919 bis heute. Köln 1989.

Epping, Volker: Die „Lex van der Lubbe“: zugleich auch ein Beitrag zur Bedeutung des Grundsatzes "nullum crimen, nulla poena sine lege“. - In: Staat 34 (1995), S. 243-267. 
Faatz, Martin: Vom Staatsschutz zum Gestapo-Terror: politische Polizei in Bayern in der Endphase der Weimarer Republik und der Anfangsphase der nationalsozialistischen Diktatur. - Würzburg 1995.

Faust, Anselm (Hrsg.): Verfolgung und Widerstand im Rheinland und in Westfalen 1933-1945. Köln 1992.

Feckler, Hans Gerhard und Volker Brüggemann (Hrsg.): Festschrift zum hundertjährigen Bestehen des Landgerichts Bochum 1892-1992. - Bochum 1992.

Feral, Thierry: Justice et nazisme. - Paris [u. a.] 1997.

Flotho, Manfred: Bruno Heusinger - ein Präsident zwischen Solidarität und Gewissen. In: Rudolf Wassermann (Hrsg.): Justiz im Wandel der Zeit. Festschrift des Oberlandesgerichts Braunschweig. - Braunschweig 1989, S. 349-369.

Förster, Michael: Jurist im Dienst des Unrechts: Leben und Werk des ehemaligen Staatssekretärs im Reichsjustizministerium, Franz Schlegelberger (1876-1970) - Baden-Baden: 1995.

Frank, Henning: Der Kampf um die freie Advokatur und die Gleichschaltung der Anwaltschaft im Dritten Reich. Zur Geschichte der Anwaltschaft Sachsens von 1831-1945. - In: Sächsisches Staatsministerium der Justiz (Hrsg.): Justiz, Juristen und politische Polizei in Sachsen 1933-1945. -Dresden 1996, S. 5-33.

Frommel, Monika: Verbrechensbekämpfung im Nationalsozialismus. - In: Michael Stolleis (Hrsg.): Die Bedeutung der Wörter. Studien zur europäischen Rechtsgeschichte. Festschrift für Sten Gagnér zum 70. Geburtstag. - München 1991, S. 47-64.

Führer, Karl Christian: Mit Juden unter einem Dach? Zur Vorgeschichte des nationalsozialistischen Gesetzes über Mietverhältnisse mit Juden. - In: 19997 (1992), H. 1, S. 51-61.

Fürst, Michael: Politisches Strafrecht im Dritten Reich: - wie das Terrorregime versuchte, Strafrecht und -justiz zum willfährigen Helfershelfer der Unterdrückung zu machen. - Aachen 1995.

Fürst, Michael: Reformen im politischen Strafrecht in der Zeit des Dritten Reiches. Unter besonderer Berücksichtigung der Sondergerichte für politische Strafsachen und deren Arbeit. - Augsburg 1990.

Godau-Schüttke, Klaus-Detlev: Justizalltag im „Dritten Reich“: zwei Urteile des Sondergerichts Kiel aus den Jahren 1943 und 1944. - In: Informationen zur Schlesw.-Holst. Zeitgeschichte 1999, 35, S. 41-63.

Godin, Hans von: Strafjustiz in rechtloser Zeit. Mein Ringen um Menschenleben in Berlin 1943-45. - Berlin 1990.

Göppinger, Horst: Juristen jüdischer Abstammung im „Dritten Reich“. Entrechtung und Verfolgung. 2., völlig neu bearb. Aufl. - München 1990.

Gostomski, Victor von [u.] Walter Loch: Der Tod von Plötzensee. Erinnerungen, Ereignisse, Dokumente, 1942-1944. - Frankfurt a. M. 1993.

Graml, Hermann: Reichskristallnacht. Antisemitismus und Judenverfolgung im Dritten Reich. München 1988.

Graue, Eugen: Das Zivilrecht im Nationalsozialismus. - In: Franz Jürgen Säcker (Hrsg.): Recht und Rechtslehre im Nationalsozialismus, Baden-Baden 1992, S. 103-124.

Gribbohm, Günter: Nationalsozialismus und Strafrechtspraxis - Versuch einer Bilanz. - In: Neue Juristische Wochenschrift 45 (1988), S. 2842-2849.

Gritschneder, Otto: Das mißbrauchte bayerische Volksgericht. - In: Lothar Gruchmann u. Reinhard Weber (Hrsg.), Der Hitler-Prozeß 1924: Wortlaut der Hauptverhandlung vor dem Volksgericht München I, T. 1, München 1997, S. XIX-XLI.

Gritschneder, Otto: „Der Führer hat Sie zum Tode verurteilt ..." Hitlers „Röhm-Putsch“-Morde vor Gericht. - München: 1993.

Gritschneder, Otto: Fachlich geeignet, politisch unzuverlässig ...: Memoiren. - München 1996.

Gritschneder, Otto: Ich predige weiter: Pater Rupert Mayer und das Dritte Reich. - Rosenheim 1987.

Gruchmann, Dietmar: Die Öffentlichkeitsarbeit der Justiz im Wandel der politischen Systeme. Eine Studie am Beispiel des Freistaates Bayern. - Garching 1994.

Gruchmann, Lothar: Das Ehegesetz vom 6. Juli 1938. Entstehung und Beurteilung. - In: Z. für Neuere Rechtsgeschichte 11 (1989), S. 63-83.

Gruchmann, Lothar u. Reinhard Weber (Hrsg.) unter Mitarbeit von Otto Gritschneder, Der Hit- 
ler-Prozeß 1924: Wortlaut der Hauptverhandlung vor dem Volksgericht München I, T.1-4, München 1997-1999.

Gruchmann, Lothar: Der Weg zum Hitler-Putsch: Das Reich und Bayern im Krisenjahr 1923. - In: ders. u. Reinhard Weber (Hrsg.), Der Hitler-Prozeß 1924: Wortlaut der Hauptverhandlung vor dem Volksgericht München I, T.1, München 1997, S. XLV-LXV.

Gruchmann, Lothar: Die "rechtsprechende Gewalt“ im nationalsozialistischen Herrschaftssystem. Eine rechtspolitisch-historische Betrachtung. - In: Wolfgang Benz [u. a.] (Hrsg.), Der Nationalsozialismus. - Frankfurt a. M. 1993, S. 78-103.

Gruchmann, Lothar: Franz Gürtner - Justizminister unter Hitler. - In: Ronald Smelser [u.a.] (Hrsg.): Die Braune Elite II. - Darmstadt 1993, S. 128-136.

Gruchmann, Lothar: Hitlers Denkschrift an die Bayerische Justiz vom 16. Mai 1923. Ein verloren geglaubtes Dokument. - In: VfZ 39 (1991), 305-338.

Gruchmann, Lothar: „Reichskristallnacht“ und Justiz im Dritten Reich. In: Neue Juristische Wochenschrift 45 (1988), S. 2856-2861.

Heinrichs, Helmut, Harald Franzki, Klaus Schmalz und Michael Stolleis (Hrsg.): Deutsche Juristen jüdischer Herkunft. - München 1993.

Helling, Wilfried: Gleichschaltung und Ausgrenzung. Der Weg der bremischen Anwaltschaft ins Dritte Reich. - Bremen 1990.

Henning, Uwe: Zum Verhältnis von Maßnahmen- und Normenstaat. Die Bedeutung des Ermittlungsverfahrens gegen Leipart und Genossen für die Machtposition der Deutschen Arbeitsfront 1933-1938. - In: ZfGWiss. 40 (1992), 176-203.

Henrichs., Horst und Karl Stephan (Hrsg.): Ein Jahrhundert Frankfurter Justiz. Gerichtsgebäude A: 1889-1989. - Frankfurt a.M. 1989.

Hensle, Michael P.: Die Todesurteile des Sondergerichts Freiburg: 1940-1945. Eine Untersuchung unter dem Gesichtspunkt von Verfolgung und Widerstand. - München 1996.

Herbert, Ulrich: [Werner] Best. Biographische Studien über Radikalismus, Weltanschauung und Vernunft; 1903-1989. - Bonn 1996.

Hetzel, Marius: Die Anfechtung der Rassenmischehe in den Jahren 1933-1939. Die Entwicklung der Rechtsprechung im Dritten Reich: Anpassung und Selbstbehauptung der Gerichte. Tübingen 1997.

Hirschberg, Max: Jude und Demokrat: Erinnerungen eines Münchener Rechtsanwalts; 1883 bis 1939. Bearb. von Reinhard Weber. - München 1998.

Hofmann, Klaus: Die Verdrängung der Juden aus öffentlichem Dienst und selbständigen Berufen in Regensburg 1933-1939. - Frankfurt a. M. 1993.

Hofmann, Ulrike Claudia: „Verräter verfallen der Feme!“. Fememorde in Bayern in den zwanziger Jahren. - Köln [u. a.] 2000.

Hornhardt, Gabriele: Strafjustiz zwischen Politik und Rechtsstaatlichkeit. Richtlinienkompetenz der Exekutive in den Jahren 1935-1953. - Hannover 1993.

Hottes, Christiane: Grauen und Normalität. Zum Strafvollzug im Dritten Reich. In: Ortstermin Hamm. Zur Justiz im Dritten Reich, hrsg. vom Oberstadtdirektor der Stadt Hamm. - Hamm 1991, S. 63-70.

Hottes, Christiane: Strafvollzug im Dritten Reich. Ein Beitrag zu seiner Darstellung und historischem Lernen aus der NS-Geschichte. - In: Juristische Zeitgeschichte 1 (1993) 169-213.

175 [Hundertfünfundsiebzig] Jahre Oberlandesgericht Oldenburg: 1814 Oberappellationsgericht, Oberlandesgericht 1989. Festschrift. - Köln [u.a.] 1989.

Institut für schleswig-holsteinische Zeit- und Regionalgeschichte (Hrsg.): Das Schleswig-Holsteinische Sondergericht Altona/Kiel 1932-1945. Ein Aktenerschließungsprojekt. - Schleswig 1995.

Institut für Zeitgeschichte (Hrsg.): Widerstand als „Hochverrat“ 1933-1945. Die Verfahren gegen deutsche Reichsangehörige vor dem Reichsgericht, dem Volksgerichtshof und dem Reichskriegsgericht. Bearb. von Jürgen Zarusky und Hartmut Mehringer. Mikrofiche-Edition. - München 1994-1998.

Jahntz, Bernhard und Volker Kähne: Der Volksgerichtshof. Darstellung der Ermittlungen der Staatsanwaltschaft bei dem Landgericht Berlin gegen ehemalige Richter und Staatsanwälte am Volksgerichtshof. - Berlin (3. Aufl.) 1992.

Jahntz, Bernhard: Diener des Unrechts: Funktionen und Selbstverständnis der NS-Strafjustiz. - In: 
Jürgen Weber und Michael Piazolo (Hrsg.): Justiz im Zwielicht. Ihre Rolle in Diktaturen und die Antwort des Rechtsstaates. - München 1998, S. 39-63.

Jakob, Anke: Nationalsozialistische Rechtsetzung am Beispiel der Verordnung zum Geltungsbereich des Strafrechts vom 6. Mai 1940. Ein Beitrag zum internationalen Strafrecht. [MikroficheAusg.]. - Marburg 1996.

Jarausch, Konrad H.: Jewish Lawyers in Germany 1848-1938. The Disintegration of a Profession. In: Yearbook Leo Baeck Institute 36 (1991), S. 171-190.

Jung, Heike und Heinz Müller-Dietz (Hrsg.): Strafvollzug im „Dritten Reich“. Am Beispiel des Saarlandes, Baden-Baden 1996.

Jureit, Ulrike: Erziehen, Strafen, Vernichten. Jugendkriminalität und Jugendstrafrecht im Nationalsozialismus. - Münster [u. a.] 1995.

Juristinnenbund e.V. (Hrsg.): Juristinnen in Deutschland. Eine Dokumentation 1900-1989. Frankfurt a.M. (2. Aufl.) 1989.

Juristinnenbund e.V. (Hrsg.): Juristinnen in Deutschland. Die Zeit von 1900 bis 1998. - (3. Aufl.) Baden-Baden 1998. [Ohne den Dokumentenanhang der 2. Aufl.].

Justizbehörde Hamburg (Hrsg.): „Für Führer, Volk und Vaterland ..." Hamburger Justiz im Nationalsozialismus. (Red.:) Klaus Bästlein, Helge Grabitz, Wolfgang Scheffler. - Hamburg 1992.

Justizbehörde Hamburg (Hrsg.): „Von Gewohnheitsverbrechern, Volksschädlingen und Asozialen ..." Hamburger Justizurteile im Nationalsozialismus. Wissenschaftl. Beirat: Helge Grabitz, Hubert Rottleuthner, Wolfgang Scheffler, (Red.:) Helge Grabitz, Wolfgang Sarodnick, Gunther Schmitz, Hamburg 1995.

Kaufmann, Rainer: Seilersbahn. Ein Weg in die Geschichte. - Bruchsal 1989.

Kebbedies, Frank: Kriminalbiologie und Jugendkriminalrecht. - Verwissenschaftlichung und Moralisierung während der NS-Zeit. - In: Juristische Zeitgeschichte 6 (1997), S. 151-167.

Keldungs, Karl-Heinz: Das Duisburger Sondergericht: 1942-1945. - Baden-Baden 1998.

Kermann, Joachim: Das Oberlandesgericht Zweibrücken und der Aufbau einer deutschen Justizverwaltung in Lothringen (1940/41). In: Sven Paulsen (Hrsg.): 175 Jahre pfälzisches Oberlandesgericht. - Neustadt an der Weinstraße 1990, S. 207-225.

Kermann, Joachim: Zur „Unabhängigkeit" der Rechtspflege während des Dritten Reiches. Vier Fallbeispiele aufgrund bisher unbekannter Lageberichte aus dem Oberlandesgerichtsbezirk Zweibrücken. - In: Mitteilungen des Historischen Vereins der Pfalz 88, 1990, S. 281-308.

Kipp, Heinrich: Rückblicke. Jurist in revolutionärem Jahrhundert. - Moers 1992.

Klee, Ernst (Hrsg.): Dokumente zur Euthanasie. - Frankfurt a. M. (13. Aufl.) 1997.

Kleßmann, Christoph: Hans Frank - Parteijurist und Generalgouverneur in Polen. - In: Ronald Smelser [u.a.] (Hrsg.): Die Braune Elite I. - Darmstadt 1989, S. 46-51.

Knobelsdorf, Andreas: Das Bielefelder Landgericht 1933-1945. - In: Juristische Zeitgeschichte 1 (1993), S. 47-101.

Knobelsdorf, Andreas, Monika Minninger und Bärbel Sunderbrink: „Das Recht wurzelt im Volk“. NS-Justiz im Landgerichtsbezirk Bielefeld. - Bielefeld 1992.

Knobelsdorf, Andreas: Politische Strafjustiz in Ostwestfalen-Lippe von 1933 bis 1945 und ihre Verarbeitung nach 1945. - In: Stadtarchiv und Landesgeschichtliche Bibliothek Bielefeld (Hrsg.): Vor braunen Richtern. Die Verfolgung von Widerstandshandlungen, Resistenz und sogenannter Heimtücke durch die Justiz in Bielefeld. - Bielefeld 1992, S. 197-270.

Koch, Hannsjoachim W.: Volksgerichtshof. Politische Justiz im 3. Reich. - München 1988.

Kohl, Wolfgang: Das Reichsverwaltungsgericht. Ein Beitrag zur Entwicklung der Verwaltungsgerichtsbarkeit in Deutschland. - Tübingen 1991.

Krach, Tillmann: Jüdische Rechtsanwälte in Preußen. Über die Bedeutung der freien Advokatur und ihre Zerstörung durch den Nationalsozialismus. - München 1991.

Krach, Tillmann: Jüdische Rechtsanwälte und ihre Vertreibung im Nationalsozialismus. - In: Recht und Politik 29 (1993), S. 85-93.

Kregel, Volker: Die Personalpolitik der Justiz im „Dritten Reich“ am Beispiel des Oberlandesgerichts Celle. - In: Ralf Dreier und Wolfgang Sellert (Hrsg.): Recht und Justiz im „Dritten Reich“ . - Frankfurt a. M. 1989, S. 226-240.

Kroeschell, Karl: Rechtsgeschichte Deutschlands im 20. Jahrhundert. - Göttingen 1992.

Kropat, Wolf-Arno: „Reichskristallnacht": der Judenpogrom vom 7. bis 10. November 1938. Urheber, Täter, Hintergründe; mit ausgewählten Dokumenten. - Wiesbaden, 1997. 
Kunz, Kerstin: Heimtückefälle vor dem Sondergericht Bielefeld 1941-1945. In: Stadtarchiv und Landesgeschichtliche Bibliothek Bielefeld (Hrsg.): Vor braunen Richtern. Die Verfolgung von Widerstandshandlungen, Resistenz und sogenannter Heimtücke durch die Justiz in Bielefeld 1933-1945. - Bielefeld 1992, S. 125-195.

Ladwig-Winters, Simone: Anwalt ohne Recht: das Schicksal jüdischer Rechtsanwälte in Berlin nach 1933. - Berlin 1998.

Lahrtz, Jens-Uwe: Zu den Strukturen und Aufgabenfeldern von politischer Polizei und Geheimer Staatspolizei in Sachsen 1933-1939. - In: Sächsisches Staatsministerium der Justiz (Hrsg.): Justiz, Juristen und politische Polizei in Sachsen 1933 bis 1945. - Dresden 1996, S. 34-65.

Landau, Peter: Die Deutschen Juristen und der nationalsozialistische Deutsche Juristentag in Leipzig 1933. - In: Z. für Neuere Rechtsgeschichte 16 (1994), S. 373-390.

Lauf, Edmund: Der Volksgerichtshof und seine Beobachter. Bedingungen und Funktionen der Gerichtsberichterstattung im Nationalsozialismus. - Opladen 1994.

Lauf, Edmund: Propaganda im Namen des deutschen Volkes: der Volksgerichtshof im Spiegel des Völkischen Beobachters. - In: Historical Social Research 21 (1996), 3, S. 76-95.

Leich, S. Hanna und André Lundt: Zur Ausschaltung jüdischer Rechtsanwälte 1933-1938 - am Beispiel Berlins. - In: Recht u. Politik 24 (1988) S. 221-229.

Leugers, Antonia: Georg Angermaier, 1913-1945. Katholischer Jurist zwischen nationalsozialistischem Regime und Kirche. Lebensbild und Tagebücher. - Mainz 1994.

Ley, Michael: „Zum Schutze des deutschen Blutes ...": „Rassenschandegesetze“ im Nationalsozialismus. - Bodenheim b. Mainz 1997.

Linck, Stephan: Der Ordnung verpflichtet: Deutsche Polizei 1933-1949: der Fall Flensburg. - Paderborn [u. a.] 2000.

Löffler, Matthias: Das Diensttagebuch des Reichsjustizministers Gürtner 1934 bis 1938: eine Quelle für die Untersuchung der „Richterdisziplinierung“ während der Anfangsjahre des Nationalsozialismus. - Frankfurt/M. [u. a.] 1997.

Ludewig, Hans-Ulrich und Dietrich Kuessner: „Es sei also jeder gewarnt ${ }_{m}$. Das Sondergericht Braunschweig 1933-1945. - Braunschweig 2000.

Lüken, Erhard-Josef: Der Nationalsozialismus und das materielle Strafrecht. Ein Beitrag zur Strafrechtsgeschichte. - Göttingen 1988.

Luge, Jens: Die Rechtsstaatlichkeit der Strafrechtspflege im Oldenburger Land, 1932-1945. - Hannover 1993.

Luge, Jens: Konflikte in der regionalen Strafrechtspflege 1932-1945. - In: 175 Jahre Oberlandesgericht Oldenburg. Festschrift. - Köln [u. a.] 1989, S. 217-251.

Majer, Diemut: Justiz und Polizei im "Dritten Reich“. - In: Ralf Dreier und Wolfgang Sellert (Hrsg.): Recht und Justiz im „Dritten Reich“. - Frankfurt a. M. 1989, S. 136-150.

Majer, Diemut: Justiz zwischen Anpassung und Konflikt am Beispiel der „Euthanasie“. - In: Ulrich Jockusch und Lothar Scholz (Hrsg.): Verantwortung von Psychiatrie und Justiz. Eine deutschisraelische Tagung. - Regensburg 1992, S. 26-40.

Marnau, Björn: Die Verfolgung von männlichen Homosexuellen im Landgerichtsbezirk Itzehoe: 1937-1945. - In: Informationen zur Schleswig-Holsteinischen Zeitgeschichte 1996, 30, S. 26-62.

Marxen, Klaus: Das Volk und sein Gerichtshof. Eine Studie zum nationalsozialistischen Volksgerichtshof. - Frankfurt a.M. 1994.

Marxen, Klaus: Der Volksgerichtshof in zeitgeschichtlicher Perspektive. - In: Juristische Zeitgeschichte 2 (1994), S. 23-37.

Marxen, Klaus: Strafrechtliche Maßnahmen zu Beginn des Dritten Reichs. Das Beispiel der Generalstaatsanwaltschaft und der Strafsenate beim Oberlandesgericht in Hamm. - In: Z. für Neuere Rechtsgeschichte 15 (1993), S. 54-65.

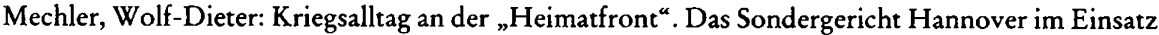
gegen „Rundfunkverbrecher", „Schwarzschlachter“, „Volksschädlinge“ und andere „Straftäter" 1939 bis 1945. - Hannover 1997.

Meyer-Höger, Maria: Der Jugendarrest. Entstehung und Weiterentwicklung einer Sanktion. - Baden-Baden 1998.

Michelberger, Hans: Berichte aus der Justiz des Dritten Reiches. Die Lageberichte der Oberlandesgerichtspräsidenten von 1940-45 unter vergleichender Heranziehung der Lageberichte der Generalstaatsanwälte. - Pfaffenweiler 1989. 
Ministerium der Justiz Rheinland-Pfalz (Hrsg.): Justiz im Dritten Reich. Justizverwaltung, Rechtsprechung und Strafvollzug auf dem Gebiet des heutigen Landes Rheinland-Pfalz (Schriftenreihe des Ministeriums der Justiz, Band 3, Teile 1 u. 2). - Frankfurt a. M. 1995.

Ministerium der Justiz Rheinland-Pfalz (Hrsg.): Justiz im Dritten Reich. NS-Sondergerichtsverfahren in Rheinland-Pfalz. Eine Dokumentation (Schriftenreihe des Ministeriums der Justiz, Band 1, Teile 1-3). - Frankfurt a. M. 1994.

Möhler, Rainer: Strafjustiz im „Dritten Reich“. - Neuerscheinungen. - In: Neue Politische Literatur 39 (1994), S. 423-441.

Möhler, Rainer: Strafvollzug im „Dritten Reich“. Nationale Politik und regionale Ausprägung am Beispiel des Saarlandes. - In: Heike Jung und Heinz Müller-Dietz (Hrsg.): Strafvollzug im „Dritten Reich“. Am Beispiel des Saarlandes. - Baden-Baden 1996, S. 9-301.

Möhler, Rainer: Volksgenossen und „Gemeinschaftsfremde“ hinter Gittern - zum Strafvollzug im Dritten Reich. - In: Zeitschrift für Strafvollzug und Straffälligenhilfe 42 (1993), S. 17-21.

Möller, Günter: Die Todesurteile des Schleswig-Holsteinischen Sondergerichts. Vertane Möglichkeiten und heutige Bemühungen um ihre Aufhebung. - In: Heribert Ostendorf (Hrsg.): Strafverfolgung und Strafverzicht. Festschrift zum 125jährigen Bestehen der Staatsanwaltschaft Schleswig-Holstein. - Köln [u. a.] 1992, S. 223-260.

Mohnhaupt, Heinz (Hrsg.): Die Rechtsgeschichte in beiden deutschen Staaten 1988-1990. Beispiele, Parallelen, Positionen. - Frankfurt a. M. 1991.

Mohr, Philipp: Hans von Dohnanyi (1902-45) - ein Jurist im Widerstand gegen Hitler. - In: Neue Juristische Wochenschrift 1995, S. $1259 \mathrm{ff}$.

Morisse, Heiko: Rechtsanwälte im Nationalsozialismus. Zur Funktion der Ehrengerichtsbarkeit, dargestellt am Beispiel des Oberlandesgerichtsbezirks Hamburg. - Hamburg 1995.

Morsey, Rudolf (Hrsg.): Das „Ermächtigungsgesetz" vom 24. März 1933. Quellen zur Geschichte und Interpretation des "Gesetzes zur Behebung der Not von Volk und Reich“. - Düsseldorf 1992.

Mühl-Benninghaus, Sigrun: Das Beamtentum in der NS-Diktatur bis zum Ausbruch des Zweiten Weltkrieges. Zu Entstehung, Inhalt und Durchführung der einschlägigen Beamtengesetze. Düsseldorf 1996.

Müller, Christian: Das Gewohnheitsverbrechergesetz vom 24. November 1933: Kriminalpolitik als Rassenpolitik. - Baden-Baden 1997.

Müller-Dietz, Heinz: Recht und Nationalsozialismus: gesammelte Beiträge. - Baden-Baden 2000.

Nagel, Irmela: Fememorde und Femeprozesse in der Weimarer Republik. - Köln 1991.

Nahmmacher, Kathrin: Die Rechtsprechung des Reichsgerichts und der Hamburger Gerichte zum Scheidungsgrund des $\ 55$ EheG 1938 in den Jahren 1938 bis 1945. - Frankfurt/M. [u. a.] 1999.

Nathans, Eli: Franz Schlegelberger. (Der Unrechts-Staat. Recht und Justiz im Nationalsozialismus, hrsg. von der Redaktion Kritische Justiz, Bd. 3.). - Baden-Baden 1990.

Nehlsen, Hermann: Der Zweite Weltkrieg in seiner Wirkung auf das Strafrecht während der NSZeit. Der Krieg als Argument. - In: Gerhard Grimm, Hermann Nehlsen und Venanz Schubert (Hrsg.): Der Zweite Weltkrieg und die Gesellschaft danach. Eine Ringvorlesung der Universität München. - St. Ottilien 1992, S. 311-362.

Nehlsen, Hermann und Georg Brun (Hrsg.): Münchener rechtshistorische Studien zum Nationalsozialismus. - Frankfurt a. M. [u.a.] 1996.

Neliba, Günter: Die vier Staatssekretäre des NS-Reichsjustizministeriums (1933-1945). - In: Die Verwaltung 27 (1994), S. 195-237.

Neugebauer, Manuala: Der Weg in das Jugendschutzlager Moringen. Eine entwicklungsgeschichtliche Analyse nationalsozialistischer Jugendpolitik. - Mönchengladbach 1997.

Niedersächsisches Justizministerium (Hrsg.): Nationalsozialistische Justiz und Todesstrafe. Eine Dokumentation zur Gedenkstätte in der Justizvollzugsanstalt Wolfenbüttel. - Braunschweig 1991.

Niermann, Hans-Eckhard: Das Landgericht Bochum in den Jahren der nationalsozialistischen Diktatur 1933-1945. - In: Hans Gerhard Feckler und Volker Brüggemann (Hrsg.): Festschrift zum hundertjährigen Bestehen des Landgerichts Bochum 1892-1992. - Bochum 1992, S. 119-152.

Niermann, Hans-Eckhard: Politische Strafjustiz im Nationalsozialismus. Exemplarische Bedingungen ihrer Durchsetzung und Radikalisierung im Dritten Reich 1933-1945. - Aachen 1996.

Niermann, Hans-Eckhard: Strafjustiz im OLG-Bezirk Hamm 1933-1945. - In: Oberstadtdirektor 
der Stadt Hamm (Hrsg.): Ortstermin Hamm. Zur Justiz im Dritten Reich. - Hamm 1991, S. 17-45.

Oberstadtdirektor der Stadt Hamm (Hrsg.): Ortstermin Hamm. Zur Justiz im Dritten Reich. Ausstellung des Stadtarchivs. - Hamm 1991.

Oehler, Christiane: Die Rechtsprechung des Sondergerichts Mannheim: 1933-1945. - Berlin 1997.

Oleschinski, Brigitte: Strafvollzug in Deutschland vor und nach 1945. Zum Problem der historischen Kontinuität in der Strafjustiz. - In: Neue Justiz 46 (1992), S. 65-68.

Ortner, Helmut: Der Hinrichter. Roland Freisler - Mörder im Dienste Hitlers. - Wien 1993.

Ostendorf, Heribert (Hrsg.): Strafverfolgung und Strafverzicht. Festschrift zum 125jährigen Bestehen der Staatsanwaltschaft Schleswig-Holstein. Köln [u. a.] 1992.

Otte, Gerhard: Die zivilrechtliche Gesetzgebung im „Dritten Reich" . - In: Neue Juristische Wochenschrift 45 (1988), S. 2836-2842.

Paul, Gerhard und Klaus-Michael Mallmann (Hrsg.): Die Gestapo. Mythos und Realität. - Darmstadt 1995.

Pauli, Gerhard: Die Rechtsprechung des Reichsgerichts in Strafsachen zwischen 1933 und 1945 und ihre Fortwirkung in der Rechtsprechung des Bundesgerichtshofes. - Berlin 1992.

Paulsen, Sven: Die Verfolgung jüdischer Richter, Beamter, Notare und Rechtsanwälte unter nationalsozialistischer Gewaltherrschaft in der Pfalz. - In: ders. (Hrsg.): 175 Jahre pfälzisches Oberlandesgericht. - Neustadt an der Weinstraße 1990, S. 267-282.

Paulsen, Sven (Hrsg.): 175 Jahre pfälzisches Oberlandesgericht. 1815 Appellationshof, Oberlandesgericht 1990. Festschrift. - Neustadt an der Weinstraße 1990.

Pientka, Andrea: Juristenausbildung zur Zeit des Nationalsozialismus. Dargestellt am Beispiel der Universität Tübingen und des OLG-Bezirks Stuttgart. - Freiburg i. Br. 1990.

Püschel, Almuth: „... der Angeklagte ist Jude“. Die Auswirkungen der antisemitischen Gesetzgebung auf Bürger der Provinz Brandenburg 1933-1945. - Potsdam 1996.

Rätsch, Birgit: Hinter Gittern. Schriftsteller und Journalisten vor dem Volksgerichtshof 1934-1945. - Bonn 1992.

Rebentisch, Dieter: Führerstaat und Verwaltung. Verfassungsentwicklung und Verwaltungspolitik. - München 1989.

Rethmeier, Andreas: „Nürnberger Rassegesetze“ und Entrechtung der Juden im Zivilrecht. Frankfurt a. M. 1995.

Rückert, Joachim: Justiz und Nationalsozialismus. Bilanz einer Bilanz. - In: Horst Möller und Udo Wengst (Hrsg.), 50 Jahre Institut für Zeitgeschichte. Eine Bilanz. - München 1999.

Rüping, Hinrich: Staatsanwaltschaft und Provinzialjustizverwaltung im Dritten Reich. Aus den Akten der Staatsanwaltschaft bei dem Oberlandesgericht Celle als höherer Reichsjustizbehörde. - Baden-Baden 1990.

Rüping, Hinrich: Zur Praxis der Strafjustiz im „Dritten Reich“. - In: Ralf Dreier und Wolfgang Sellert (Hrsg.): Recht und Justiz im „Dritten Reich“. - Frankfurt a.M. 1989, S. 180-193.

Rüthers, Bernd und Martin Schmitt: Die juristische Fachpresse nach der Machtergreifung der Nationalsozialisten. - In: Juristenzeitung 43 (1988), S. 369-377.

Rüthers, Bernd: Entartetes Recht. Rechtslehren und Kronjuristen im Dritten Reich. - München (2. Aufl.) 1989.

Rüthers, Bernd: Recht als Waffe des Unrechts - Juristische Instrumente im Dienst des NS-Rassenwahns. - In: Neue Juristische Wochenschrift 45 (1988), S. 2825-2836.

Rüthers, Bernd: Von der „Normalität“ der Ziviljustiz im Dritten Reich. - In: Zeitschrift für Neuere Rechtsgeschichte 11 (1989), S. 184-191.

Rumschöttel, Hermann: Das Bayerische Staatsministerium der Justiz 1799-1966. - München 1990.

Sächsisches Staatsministerium der Justiz (Hrsg.): Justiz, Juristen und politische Polizei in Sachsen 1933 bis 1945. Gehorsam und Vorbehalte. - Dresden 1996.

Säcker, Franz Jürgen (Hrsg.): Recht und Rechtslehre im Nationalsozialismus. Ringvorlesung der Rechtswissenschaftlichen Fakultät der Christian-Albrechts-Universität zu Kiel. - Baden-Baden 1992.

Sarodnick, Wolfgang: „Dieses Haus muß ein Haus des Schreckens werden ...“. Strafvollzug in Hamburg 1933 bis 1945. - In: Justizbehörde Hamburg (Hrsg.). „Für Führer, Volk und Vaterland ...“. Hamburger Justiz im Nationalsozialismus. - Hamburg 1992, S. 332-381. 
Schenk, Dieter: Die Post von Danzig: Geschichte eines deutschen Justizmords. - Reinbek bei Hamburg 1995.

Schiller, Christof: Das Oberlandesgericht Karlsruhe im Dritten Reich. - Berlin 1997.

Schlüter, Holger: Die Urteilspraxis des nationalsozialistischen Volksgerichtshofs. - Berlin 1995.

Schmidt, Herbert: „Beabsichtige ich die Todesstrafe zu beantragen“. Die nationalsozialistische Sondergerichtsbarkeit im Oberlandesgerichtsbezirk Düsseldort 1933-1945. - Essen 1998.

Schmidt, Jörg: Otto Koellreutter: 1883-1972; sein Leben, sein Werk, seine Zeit. - Frankfurt am Main u. a. 1995.

Schmitz, Gunther: Die Vor- und Nachschaubesprechungen in Hamburg 1942-1945. Zur Justizlenkung im totalen Krieg. - In: Justizbehörde Hamburg (Hrsg.): „Von Gewohnheitsverbrechern, Volksschädlingen und Asozialen ...". Hamburger Justizurteile im Nationalsozialismus. - Hamburg 1995, S. 447-470.

Schorn, Franz Hubert: Franz Virnich 1882-1943. Opfer der NS-Justiz. Bericht und Dokumentation. - Landwaden 1998.

Schreiber, Hans-Ludwig: Die Strafgesetzgebung im „Dritten Reich“. - In: Ralf Dreier und Wolfgang Sellert (Hrsg.): Recht und Justiz im „Dritten Reich“. - Frankfurt a. M. 1989, S. 151-179.

Schroeder, Friedrich-Christian und Jens-Uwe Lahrtz: Die nationalsozialistischen Sondergerichte in Sachsen 1933-1945. In: Sächsisches Staatsministerium der Justiz (Hrsg.): Justiz, Juristen und politische Polizei in Sachsen 1933 bis 1945. - Dresden 1996, S. 66-109.

Schröder, Rainer: „... aber im Zivilrecht sind die Richter standhaft geblieben!“ Die Urteile des OLG Celle aus dem Dritten Reich. - Baden-Baden 1988.

Schröder, Rainer: Die Bewältigung des Dritten Reiches durch die Rechtsgeschichte. - In: Heinz Mohnhaupt (Hrsg.), Die Rechtsgeschichte in beiden deutschen Staaten 1988-1990. Beispiele, Parallelen, Positionen. - Frankfurt a. M. 1991, S. 604-647.

Schubert, Werner, Werner Schmid und Jürgen Regge (Hrsg.): Akademie für Deutsches Recht 1933-1945. Protokolle der Ausschüsse, Bände I-IV Berlin 1986-1996; Werner Schubert (Hrsg.): Bände V-X, Frankfurt a.M. 1997-2000.

Schubert, Werner (Hrsg.): Das Familien- und Erbrecht unter dem Nationalsozialismus. Ausgewählte Quellen zu den wichtigsten Gesetzen und Projekten aus den Ministerialakten. - Paderborn 1993.

Schubert, Werner [u. a.] (Hrsg.): Quellen zur Reform des Straf- und Prozeßrechts. - Berlin. Abt. 2: NS-Zeit (1933-1939) - Strafgesetzbuch. Band 1: Entwürfe eines Strafgesetzbuchs, hrsg. von Jürgen Regge und Werner Schubert, Teil 1 (1988), Teil 2 (1990). - Band 2: Protokolle der Strafrechtskommission des Reichsjustizministeriums, hrsg. von Jürgen Regge und Werner Schubert, Teil 1 (1988), Teil 2 (1989) Teil 3 (1990), Teil 4 (1994). Abt. 3: NS-Zeit (1933-1939) - Strafverfahrensrecht. Band 1: Entwürfe zu einer Strafverfahrensordnung und einer Friedens- und Schiedsrichterordnung (1936-1939), hrsg. von Werner Schubert (1991). Band 2: Protokolle der Großen Strafprozeßkommission des Reichsjustizministeriums (1936-1938), hrsg. von Werner Schubert, Teil 1 (1991), Teil 2 (1992), Teil 3 (1993).

Schudnagies, Christian: Hans Frank. Aufstieg und Fall des NS-Juristen und Generalgouverneurs. Frankfurt a.M. 1989.

Schücking, Prosper und Martin Sölle: $\ 175$-Strafrechtliche Verfolgung homosexueller Männer in Köln. - In: Cornelia Limpricht, Jürgen Müller und Nina Oxenius (Hrsg.): „Verführte“ Männer. Das Leben der Kölner Homosexuellen im Dritten Reich. - Köln 1991, S. 104-119.

Schütze, Steffen: Sondergerichtsbarkeit in Deutschland am Beispiel des Sondergerichts für das Land Sachsen mit Sitz in Freiberg (Magisterarbeit). - Leipzig 1995.

Schwartz, Michael: Bauern vor dem Sondergericht. Resistenz und Verfolgung im bäuerlichen Milieu Westfalens. - In: Anselm Faust (Hrsg.). Verfolgung und Widerstand im Rheinland und in Westfalen 1933-1945, Köln 1992, S. 113-123.

Schwarz, Alfons: Rechtsprechung durch Sondergerichte. Zur Theorie und Praxis im Nationalsozialismus am Beispiel des Sondergerichts Berlin. - Augsburg 1992.

Seeger, Andreas und Fritz Treichel: Hinrichtungen in Hamburg und Altona 1933-1944: „in einer schlagkräftigen Strafrechtspflege müssen Todesurteile unverzüglich vollstreckt werden“. - Hamburg 1998 .

Seibert, Winfried: Das Mädchen, das nicht Esther heißen durfte. Eine exemplarische Geschichte. Leipzig: 1996. 
Simon, Jürgen: Die Erbgesundheitsgerichtsbarkeit im OLG-Bezirk Hamm. Rechtsprechung zwischen juristischen Vorgaben und ideologischen Anforderungen. - In: Juristische Zeitgeschichte 1 (1993) S. 131-167.

Simon, Jürgen: Kriminalbiologie im Dritten Reich: Kriminalbiologie - theoretische Konzepte und praktische Durchführung eines Ansatzes zur Erfassung von Kriminalität. - In: Juristische Zeitgeschichte 6 (1997), S. 69-105.

Siol, Joachim: Justiz und Tagespresse in der NS-Zeit. - In: 175 Jahre Oberlandesgericht Oldenburg. Festschrift, Köln [u. a.] 1989, S. 323-336.

Sparing, Frank: „... wegen Vergehen nach $₫ 175$ verhaftet“: die Verfolgung der Düsseldorfer Homosexuellen während des Nationalsozialismus. Düsseldorf 1997.

Sparing, Frank: Zwangskastrationen im Nationalsozialismus. Das Beispiel der Kriminalbiologischen Sammelstelle Köln. - In: Juristische Zeitgeschichte 6 (1997), S. 169-212.

Staudinger, Roland: Politische Justiz. Die Tiroler Sondergerichtsbarkeit im Dritten Reich am Beispiel des Gesetzes gegen heimtückische Angriffe auf Partei und Staat. - Schwaz 1994.

Stein-Stegemann, Hans-Konrad: In der „Rechtsabteilung“ des „Unrechts-Staates“. Richter und Staatsanwälte in Hamburg 1933-1945. - In: Justizbehörde Hamburg (Hrsg.): „Für Führer, Volk und Vaterland ...". Hamburger Justiz im Nationalsozialismus. - Hamburg 1992, S. 146-215.

Steiner, John M., Jobst Freiherr von Cornberg: Willkür in der Willkür: Befreiungen von den antisemitischen Nürnberger Gesetzen. - In: VfZ 46 (1998), S. 143-187.

Stolleis, Michael: Geschichte des öffentlichen Rechts in Deutschland. Bd. 3. Staats- und Verwaltungsrechtswissenschaft in Republik und Diktatur. 1914-1945. - München 1999.

Stolleis, Michael: Nationalsozialistisches Recht. - In: Handwörterbuch zur deutschen Rechtsgeschichte, Bd. 3. Berlin 1989, Sp. 873-892.

Stolleis, Michael: Recht im Unrecht. Studien zur Rechtsgeschichte des Nationalsozialismus. Frankfurt a.M. 1994.

Streim, Alfred: Zur Bildung und Tätigkeit der Sondergerichte, in: Formen des Widerstandes im Südwesten 1933-1945. Scheitern und Nachwirken, hrsg. von der Landeszentrale für politische Bildung Baden-Württemberg und vom Haus der Geschichte Baden-Württemberg durch Thomas Schnabel. Ulm 1994, S. 237-258.

Strohm, Christoph: Theologische Ethik im Kampf gegen den Nationalsozialismus. Der Weg Dietrich Bonhoeffers mit den Juristen Hans von Dohnanyi und Gerhard Leibholz in den Widerstand. - München 1989.

Süß, Winfried: Krankenmord. Forschungsstand und Forschungsfragen zur Geschichte der nationalsozialistischen „Euthanasie“. - In: Theresia Bauer und Winfried Süß (Hrsg.): NS-Diktatur, DDR, Bundesrepublik. Drei Zeitgeschichten des vereinigten Deutschland. Werkstattberichte. Neuried 2000, S. 47-86.

Sunnus, Michael: Der NS-Rechtswahrerbund (1928-1945). Zur Geschichte der nationalsozialistischen Juristenorganisation. - Frankfurt a. M. 1990.

Tarrab-Maslaton, Martin: Rechtliche Strukturen der Diskriminierung der Juden im Dritten Reich. Berlin 1993.

Thamer, Hans-Ulrich: Justiz und Politik im Dritten Reich. - In: Oberstadtdirektor der Stadt Hamm (Hrsg.): Ortstermin Hamm. Zur Justiz im Dritten Reich. - Hamm 1991, S. 12-16.

Tilitzki, Christian: Alltag in Ostpreußen 1940-1945. Die geheimen Lageberichte der Königsberger Justiz 1940-1945. - Leer 1991.

Toppe, Andreas: Provinzialjustizverwaltung, Richter und Urteilspraxis im Dritten Reich. Zur Geschichte des Oberlandesgerichts München. - (Magisterarbeit) Augsburg 1995.

Unger, Manfred: Die Leipziger Sondergerichte 1940-1945 und der Volkswiderstand in Westsachsen. - In: Hans-Dieter Schmid (Hrsg.): Zwei Städte im Hakenkreuz. Widerstand und Verweigerung in Hannover und Leipzig 1933-1945. - Leipzig 1994, S. 178-196.

Unger, Manfred: Zu Entwicklung und Struktur der Leipziger Sonderjustiz 1940-1945. - In: Marlis Buchholz, Claus Füllberg-Stolberg und Hans-Dieter Schmid (Hrsg.): Nationalsozialismus und Region. Festschrift für Herbert Obenaus zum 65. Geburtstag. - Bielefeld 1996, S. 227-239.

Vormbaum, Thomas: Aktuelle Bezüge nationalsozialistischer Strafgesetzgebung. - In: Heribert Ostendorf (Hrsg.): Strafverfolgung und Strafverzicht. Festschrift zum 125jährigen Bestehen der Staatsanwaltschaft Schleswig-Holstein. - Köln [u. a.] 1992, S. 71-91.

Vormbaum, Thomas: Beiträge zur juristischen Zeitgeschichte. - Baden-Baden 1999. 
Voß, Ingo von: Die Verwaltungsgerichtsbarkeit in Hamburg von 1921 bis 1945. Die Entwicklung vor und nach dem Entstehen nationalsozialistischer Tendenzen in Gesetzgebung und Rechtsprechung. - Witterschlick/Bonn 1988.

Vortmann, Jürgen: Juristischer Widerstand gegen den Nationalsozialismus. - In: Z. für Rechtspolitik 23 (1990), 193-198.

Wacker, Gerd: Der Erbrechtsausschuß der Akademie für Deutsches Recht und dessen Entwurf eines Erbgesetzes. - Frankfurt /M. [u. a.] 1997.

Wagner, Heinz: Das Strafrecht im Nationalsozialismus. - In: Franz Jürgen Säcker (Hrsg.). Recht und Rechtslehre im Nationalsozialismus, Baden-Baden 1992, S. 141-184.

Wagner, Patrick: Volksgemeinschaft ohne Verbrecher: Konzeptionen und Praxis der Kriminalpolizei in der Zeit der Weimarer Republik und des Nationalsozialismus. - Hamburg 1996.

Waller, Helmut: Das Wirken der Sondergerichte. - In: Justizministerium Baden-Württemberg und Landeszentrale für politische Bildung Baden-Württemberg (Hrsg.): Recht im Nationalsozialismus. Bericht über die Tagung vom 5. bis 8. November 1990 in St. Johann-Lonsingen. - Stuttgart 1993, S. 90-114.

Walther, Manfred: Arenen politischer Justiz: Sondergerichtsbarkeit. - In: Axel Görlitz (Hrsg.), Politische Justiz. - Baden-Baden 1996, S. 31-90.

Walther, Manfred: Hat der juristische Positivismus die deutschen Juristen im „Dritten Reich“ wehrlos gemacht?. - In: Ralf Dreier und Wolfgang Sellert (Hrsg.): Recht und Justiz im „Dritten Reich“. - Frankfurt a. M. 1989, S. 323-354.

Wassermann, Rudolf: Auch die Justiz kann aus der Geschichte nicht aussteigen. Studien zur Justizgeschichte. - Baden-Baden 1990.

Wassermann, Rudolf (Hrsg.): Justiz im Wandel der Zeit. Festschrift des Oberlandesgerichts Braunschweig. - Braunschweig 1989.

Wassermann, Rudolf: Zur Geschichte des Oberlandesgerichts Braunschweig. - In: ders. (Hrsg.): Justiz im Wandel der Zeit. Festschrift des Oberlandesgerichts Braunschweig. - Braunschweig 1989, S. 11-110.

Wehbecker, Gerd: Zwischen Freispruch und Todesstrafe: die Rechtsprechung der nationalsozialistischen Sondergerichte Frankfurt/Main und Bromberg. - Baden-Baden 1998.

Weinke, Wilfried: The Persecution of Jewish Lawyers in Hamburg. A Case Study: Max Eichholz and Herbert Michaelis. - In: Yearbook Leo Baeck Institute 42 (1997), S. 221-237.

Weiß, Hermann (Hrsg.): Biographisches Lexikon zum Dritten Reich. - Frankfurt a. M. 1998.

Weiß, Konrad: Lothar Kreissig. Prophet der Versöhnung. - Gerlingen 1998.

Weitzel, Jürgen: Sonderprivatrecht aus konkretem Ordnungsdenken: Reichserbhofrecht und allgemeines Privatrecht. - In: Z. für Neuere Rechtsgeschichte 14 (1992), S. 55-79.

Wenzel, Gerhard: Die Darmstädter Justiz 1933. Chronik einer Metamorphose. - In: Justiz-Report Landgericht Darmstadt. - Darmstadt 1992, S. 175-195.

Werle, Gerhard: Das Strafrecht als Waffe. Die Verordnung gegen Volksschädlinge vom 5. September 1939. In: Juristische Schulung 29 (1989), S. 952-958.

Werle, Gerhard: Justiz-Strafrecht und polizeiliche Verbrechensbekämpfung im Dritten Reich. Berlin 1989.

Werle, Gerhard: Zur Reform des Strafrechts in der NS-Zeit. Der Entwurf eines Deutschen Strafgesetzbuchs 1936. - In: Neue Juristische Wochenschrift 45 (1988), S. 2865-2867.

Wieland, Günther: Das war der Volksgerichtshof. Ermittlungen, Fakten, Dokumente. - Berlin (Ost) 1989.

Willems, Susanne: Lothar Kreyssig: vom eigenen verantwortlichen Handeln. Eine biographische Studie zum Protest gegen die Euthanasieverbrechen in Nazi-Deutschland. - Berlin [1995].

Willoweit, Dietmar: Das völkische Recht und der SS-Staat. Die Reden des Hans Frank vom Sommer 1942. - In: Z. für Neuere Rechtsgeschichte 16 (1994), S. 272-296.

Wolff, Jörg: Jugend und Strafrecht im Nationalsozialismus. - In: Z. für Neuere Rechtsgeschichte 13 (1991) S. 41-66.

Wolff, Jörg unter Mitarb. von Christine Dörner, Frank Kebbedies [u. a.]: Jugendliche vor Gericht im Dritten Reich. Nationalsozialistische Jugendstrafrechtspolitik und Justizalltag. - München 1992.

Wolff, Jörg: Justizverwaltung im Bezirk des OLG Oldenburg 1933-1945. - In: 175 Jahre Oberlandesgericht Oldenburg. Festschrift. Köln u. a. 1989, S. 289-321. 
Wrobel, Hans: Schlegelberger und seine Biographen. Kritische Anmerkungen zu zwei Sichtweisen einer Person. - In: Ius Commune. Z. für Europäische Rechtsgeschichte XX (1993), S. 273-289.

Wrobel, Hans: Strafjustiz im totalen Krieg. Aus den Akten des Sondergerichts Bremen 1940 bis 1945, Band 1-3, hrsg. vom Senator für Justiz und Verfassung der Freien Hansestadt Bremen. Bremen 1991/1994.

Wüllenweber, Hans: Sondergerichte im Dritten Reich. Vergessene Verbrechen der Justiz. - Frankfurt a. M. 1990.

Wulff, Arne: Staatssekretär Prof. Dr. Dr. H.c. Franz Schlegelberger, 1876-1970. - Frankfurt a.M. 1991.

Zarusky, Jürgen: Politische Strafjustiz im nationalsozialistischen Doppelstaat. - In: Jürgen Weber und Michael Piazolo (Hrsg.): Justiz im Zwielicht. Ihre Rolle in Diktaturen und die Antwort des Rechtsstaates. - München 1998, S. 25-38.

Zeidler, Manfred: Das Sondergericht Freiberg. Zu Justiz und Repression in Sachsen 1933-1940. Dresden 1998.

Ziegler, Hans: 175 Jahre Oberlandesgericht Zweibrücken. 1815-1990. Seine Richter und Staatsanwälte. - In: Sven Paulsen (Hrsg.): 175 Jahre pfälzisches Oberlandesgericht. - Neustadt an der Weinstraße 1990, S. 411-437.

Zirker, Matthias: Vertrag und Geschäftsgrundlage in der Zeit des Nationalsozialismus. - Würzburg 1996. 


\section{Periodica}

Amtsblatt des Gaues Berlin der NSDAP 1938 Der Angriff 1933-36

Anwaltsblatt 1983

Archiv für die civilistische Praxis 1963

Baseler Nationalzeitung 1919

Bayerische Staatszeitung 1933

Bayerischer Kurier 1931

Bayerischer Staatsanzeiger 1933

Bayerisches Justizministerialblatt, Neue Folge, $1928 \mathrm{ff}$

Befehlsblatt des Chefs Sipo/SD, $1940 \mathrm{ff}$.

Berliner Börsen-Zeitung 1935

Berliner Tageblatt 1935

Bourse Egyptienne 1934

Coburger Zeitung 1929

Deutsche Allgemeine Zeitung 1933

Deutsche Juristenzeitung 1924, 1934, 1935

Deutsche Justiz 1933-45

Der Deutsche Justizbeamte 1938

Der Deutsche Polizeibeamte 1934, 1935

Der deutsche Rechtspfleger 1934

Deutsche Richterzeitung $1933 \mathrm{ff}$.

Deutsche Verwaltung 1944

Deutsches Gemein- und Wirtschaftsrecht 1937, 1940

Deutsches Recht $1933 \mathrm{ff}$.

Deutsches Strafrecht $1934 \mathrm{ff}$.

Entscheidungen des Reichsgerichts in Strafsachen $1933 \mathrm{ff}$.

Entscheidungen des Reichsgerichts in Zivilsachen $1933 \mathrm{ff}$.

Fränkischer Kurier 1941

Frankfurter Allgemeine Zeitung 1963

Frankfurter Zeitung 1933

Der Gerichtssaal 1934, 1935

Gesetzblatt für die Freie Stadt Danzig 1935

Gesetz- und Verordnungsblatt für den Freistaat Bayern $1933 \mathrm{ff}$.

Göttinger Jahrbuch 1980

Goltdammer's Archiv für Strafrecht 1984

Grafinger Zeitung 1931

Hamburger Gesetzes- und Verordnungsblatt 1933

Hamburger Jahrbuch für Wirtschafts- und Gesellschaftspolitik 1960

Hamburger Justizverwaltungsblatt 1933

Handbuch für den Preußischen Staat 1933 ff.

Die Innere Mission 1947
Jahrbuch der Akademie für Deutsches Recht 1933-40

Jahrbuch Deutsches Recht 1933 ff.

Jahrbuch für westdeutsche Landesgeschichte 1979,1980

The Journal of criminal law and criminology 1936

Juristenzeitung $1951 \mathrm{ff}$.

Juristische Arbeitsblätter 1977

Juristische Rundschau $1947 \mathrm{ff}$.

Juristische Schulung 1961 ff.

Juristische Wochenschrift 1933-39

Die Justiz 1927/28

Justizministerialblatt für den Freistaat Bayern $1919 \mathrm{ff}$.

Kalender für Reichsjustizbeamte 1936-1941

Keesings Archiv der Gegenwart 1935

Kölner Zeitschrift für Soziologie und Sozialpsychologie 1958

Kritische Justiz $1968 \mathrm{ff}$.

Leipziger Zeitschrift für Deutsches Recht 1928

Miesbacher Anzeiger 1934

Ministerial-Blatt für die preußische innere Verwaltung 1933-35

Ministerial-Blatt des Reichs- und Preußischen Ministeriums des Inneren $1936 \mathrm{ff}$.

Mitteilungen der Internationalen Kriminalistischen Vereinigung, Neue Folge 1933

Mitteilungen der Reichs-Rechtsanwaltskammer 1939

Mitteilungen über die SS- und Polizeigerichtsbarkeit 1940

Mitteilungsblatt des Bundes National-Sozialistischer Deutscher Juristen/Nationalsozialistischer Rechtswahrerbund $1935 \mathrm{ff}$.

Monatsschrift für Kriminalbiologie und Strafrechtsreform 1939

München-Augsburger Abendzeitung 1931

Münchener Post 1931

Münchener Zeitung 1934

Münchner Neueste Nachrichten 1924, 1932

Neues Tageblatt (Waldenburg) 1934

Das Parlament, einschl. Beilage 1985

Der Parteirichter 1936

Preußische Gesetzessammlung $1919 \mathrm{ff}$.

Recht und Politik 1983

Rechtshistorisches Journal 1985 
Reichsgesetzblatt, Teil I, 1922 ff.

Reichsministerialblatt 1930

Reichsverwaltungsblatt 1935

Schulthess' Europäischer Geschichtskalender $1932 \mathrm{ff}$.

Das Schwarze Korps 1933-42

Statistisches Jahrbuch für das Deutsche Reich 1933-42

Der Stürmer $1933 \mathrm{ff}$.

Süddeutsche Juristenzeitung 1946-50

Tempo 1930

Verhandlungen des Bayerischen Landtags.

Stenographische Berichte 1919-29

Verhandlungen des Reichstags. Stenographische Berichte $1924 \mathrm{ff}$.

Verordnungsblatt der Reichsleitung der NSDAP $1933 \mathrm{ff}$.
Vierteljahrshefte für Zeitgeschichte 1953 ff.

Völkischer Beobachter 1920-41

Vorwärts 1922, 1924, 1926

Vossische Zeitung 1929, 1933

Zeitschrift der Akademie für deutsches Recht $1934 \mathrm{ff}$.

Zeitschrift für bayerische Landesgeschichte 1985

Zeitschrift für die gesamte Strafrechtswissenschaft 1923, $1933 \mathrm{ff}$.

Zeitschrift für die Geschichte des Oberrheins 1981

Zeitschrift für Geschichtswissenschaft 1953

Zeitschrift für Neuere Rechtsgeschichte $1979 \mathrm{ff}$.

Zeitschrift für Rechtspolitik $1968 \mathrm{ff}$. 


\section{Personenregister}

Franz Gürtner und die in den Geschäftsverteilungsplänen (Anlagen 1 und 2 des Anhangs) enthaltenen Namen wurden nicht aufgenommen.

Ablaß, Bruno 85

Adler, Julius 440,464

Allers, Dietrich 524

Alpers, Friedrich 206, 240, 271

Alquen, Gunter d' 1003

Altmann, Josef 641

Altmeyer, Josef 223, 261

Aly, Götz 534

Am Zehnhoff, Hugo 323

Anderbrügge, Klaus 2

Anders, Georg 246

Anz, Dr. (Oberlandesgerichtspräsident in

Kassel) 225, 1212

Aron, Wilhelm 634

Auer, Erhard 75

Aull, Hans 36f., 39f., 236, 1211

Bach, Georg 226, 1211

Bähr, Dr. (Präsident in Kassel) 935, 981

Bälz, Rudolf 245

Bär, Kurt $404 \mathrm{f}$.

Ballerstedt, Otto 439 f., 459

Barnickel, Paul 645

Barth, Heinrich 68

Bartholomä (Ortsgruppenleiter der NSDAP in St. Pauli) 378

Bauer, Karl $276 \mathrm{f}$.

Bayer, Barbara 17

Beauvais, Peter 367

Beck, Friedrich 439f., 459

Beck, Jakob 410

Beck, Leonhard 39, 42

Becker, Bruno 1212

Becker, Friedrich 236, 1214

Becker, Ludwig 226, 283, 1213

Behrends, Hermann 311

Beier, Leo 261

Below, Nicolaus v. 1071

Bems, Emil 285, 1213

Benario, Rudolf 634

Bender, Arthur 244
Benninghoven, Friedrich 7

Bepler, Hans-Joachim 625

Bergbohm, Max 132

Bergmann, Alexander 529f., 1213

Berkowitz, Horst 185

Bertram, Adolf 498, 513

Bertram, Otto 236, 274, 285, 1213

Best, Werner 311,560 ff., $570,581,592 \mathrm{ff}$., $598,614,630,677,701 \mathrm{f}$., 708,715 f., 718 f., 888,1020

Betz, Hanns $413 \mathrm{f}$.

Beyer, Rudolf 279, 282, 1212

Biederbick, Karl-Heinz 7

Bilfinger, Carl 61

Binding, Karl 497

Binz, Gerhard-Ludwig 766

Bismarck, Herbert v. 325

Bistritschan, Wilhelm 261

Blankenburg, Werner 523

Blomberg, Werner v. $68,75,94,158 \mathrm{f} ., 323$, $325,450,483,802,833,945,959$

Bodelschwingh, Friedrich v. 506

Bodenschatz, Karl 964

Böhme, Hermann $417 \mathrm{f}$.

Bohla, Friedrich 11

Bohne, Gerhard 499, 503, 509 f., 524, $529 \mathrm{f}$., 533

Boldt, Gottfried 1086

Bormann, Albert 201

Bormann, Martin 115, 187, 201, 203, 205, 207, 210, 212, 216, 256 ff., 266, 269, 273, $282,319,377,379,502,521,683,687 \mathrm{f}$., $748,751,783,849,859,946,1015,1141$

Bose, Herbert v. 443, 447, 449, 455, 458, $479 \mathrm{f}$.

Bouhler, Philipp $192 \mathrm{f} ., 219,257,373,379$, 469,500 ff., 507 ff., 516,523 f., 527 f., 533 , $652,708 \mathrm{f} ., 1123$

Bracht, Fritz 193

Brack, Viktor 500, 502, 504, 506, 509 f., 512, 520, 523 f., 526 ff., 533, 709 
Brandis, Ernst 234, 258

Brandmüller, Hermann 16,1111

Brandt, Karl 499, 501 ff., 512, 524, 528, 533

Brauchitsch, Manfred v. 72

Braun, Konrad („Barum“) 223

Braun, Magnus Frhr. v. 72, 455

Braun, Otto 59, 225

Braune, Paul 506, 510

Brauneis, Max $39 \mathrm{f}$.

Brecht, Arnold 60

Brederek (Oberamtsanwalt) 1002

Bredow, Ferdinand 442, 450, 458

Bredt, Johann Viktor 253

Breithaupt, Franz 479

Brentano, Lujo (Ludwig Josef) 11, 13

Brettle, Emil 240, 283, 882 f., 1079f., 1082, $1086 \mathrm{f} ., 1212$

Bretzfeld, Friedrich 53, 73, 82

Broszat, Martin 7

Brückner, Helmuth 462 f., 465

Brückner, Wilhelm $33,42,881 \mathrm{f}$.

Brüning, Heinrich 9, 55, 58, 85, 241

Bruner, Wilhelm $961 \mathrm{f} ., 965 \mathrm{f}$., $969 \mathrm{f}$.

Brunner, Dr. (Erster Oberstaatsanwaltstellvertreter in Wien) 1214

Buch, Walter $377 \mathrm{f} ., 492,532 \mathrm{f}$.

Buchheit, Gert 2

Büchner, Fritz 75

Bühler, Josef $83,233,235,261 \mathrm{f}$.

Bürckel, Josef $178,214,260,263,380,384$

Bürger, Ludwig 223

Bürk, Fritz 642

Bürkle, Fritz 280, 1214

Bullerjahn, Walter 714

Bumke, Erwin 79, 121, 126, 131, 165, 242, 253, 671, 826, 1012, 1077 f., 1087, 1101

Bumke, Oswald 79

Burkhardt, Friedrich 236, 1213

Burmeister, Heinrich 239, 272, 1214

Busch, Ernst 244, 258, 676

Busch Becerra, German 676

Busley, Hermann-Joseph 7

Buttmann, Rudolf 24

Buzengeiger, Karl 240, 275, 1212

Calonder, Felix 266

Cammerer, Clemens 232

Castner, Karl 183, 232, 239

Chamberlain, Arthur Neville 179

Charig (Kaufmann in Hirschberg) 460, 464, 480

Christians, Rudolf 1213

Conti, Leonardo $503,507,516,523$

Cormann, Paul 225, 1214

Corsing, Fritz 223

Cossmann, Heinrich 75
Creutzfeldt, Kurt 247

Crohne, Wilhelm 106, 141, 219, 223, 244, $247,359,362,518,523 \mathrm{f} ., 527,539$ ff., 570 , $578,585,593$ ff., $601 \mathrm{f} ., 605,607 \mathrm{ff} ., 611 \mathrm{f}$., $614,617 \mathrm{ff} ., 624,626,628,651,680,682 \mathrm{f}$., $685 \mathrm{ff} ., 713 \mathrm{ff} ., 718,727,734 \mathrm{ff}, 754,760$, 765, 882, 885, 916 f., 919,932 f., 950,964 , 981, 983, 994, 1015, 1020, 1077 ff., 1084 ff., 1094, 1099, 1100 f., 1103, 1105, 1107

Cuhorst, Albert 932, 981

Cuno, Wilhelm 58

Däubler, Helmut 246

Dahm, Georg 311, 754, 760, 765, $771 \mathrm{f} ., 790$, 850, 858 f., 1015, 1034 ff., 1139

Daluege, Kurt 222f., 353, 709, $720 \mathrm{ff}$.

Danckwortt (Generalstaatsanwalt in Königsberg) 226

David, Alfons $126 \mathrm{f} ., 165$

David, Herbert 278, 1213

Degen, Richard 231

Delitzsch, Kurt 1212

Dengler, Hans-Herbert 350

Detlefs, Walther 935, 981

Detten, Georg v. 458

Dibelius, Otto $76 \mathrm{f}$.

Diels, Rudoif 324, 330, 345f., 349f., 352, $363,380,434,462,831$

Dietrich, Josef (Sepp) 435 ff., 446f., 454, 478

Dietz, Heinrich (ORRat im RJM) 244

Dietz, Heinrich (MRat im RKriegsM) 1015

Dillinger, John H. $644 \mathrm{f}$.

Dimitroff, Georgi 957

Dittmann, Wilhelm 26

Dittrich, Gerhard 246, 262

Dix, Rudolf 75 f., 323, 566

Dodd, William Edward 476

Döbig, Friedrich August 232, 239, 274, 282 , $285,330,392,401,403 \mathrm{f}$., $406 \mathrm{ff}$., $435 \mathrm{ff}$., $477,637,639,643 \mathrm{f} ., 1213$

Döll, Nikolaus $236 \mathrm{f}$., 1211

Dörffler, Fritz 244, 935, 941, 981, 983, 994

Doerner, Karl 243, 245f., 981, 1055

Dohna, Alexander Graf zu 34

Dohnanyi, Christine v. $73,78 \mathrm{ff} ., 253 \mathrm{f}$.

Dohnanyi, Hans v. 16 f., 71, 73, 76, 78 ff., 106, $195,202,243,245$ f., 253 ff., 377,416 ff., $446,456,462,480,506,557$ f., 650, 769, 832

Dollfuß, Engelbert 261, 278, 288

Draeger, Max 275f., 281, 1213

Drendel, Karl 287, 1214

Drescher, Erich 240, 949, 1081, 1212

Drescher, Friedrich 232, 239, 245, 259

Dresse, Martin 30f., 36 f., 39, 43

Dressel, Friedrich 634

Droege, Heinrich 176f., 189, 1006 
Dürig, Ernst $276 \mathrm{f}$., 1211

Dürr, Alfred 22, 28, 30, 34, 43, 232, 239, 274, $330,332,754,769,835,837,933,935$, 980 f., 1019 f., 1213

Dusenschön, Willy $374 \mathrm{ff}$.

Eberl, Irmfried 509

Eberstein, Friedrich Karl Frhr. v. 442

Ebert, Friedrich (Ministerialrat) 246, 261

Ebert, Friedrich (Reichspräsident) 24, 32

Echterhölter, Rudolf 1

Eckert, Gerhard 240, 1211

Eckert, Hans 657

Eckhardt, Karl August 1139

Eggerstedt, Otto 363

Ehard, Hans 36, 45, 232

Ehrhardt, Arthur 225, 1212

Ehrhardt, Hermann 33, 35

Eichler, Johannes 246, 259, 969

Eicke, Theodor $364 \mathrm{ff} ., 437,478,638,641$, $645 \mathrm{ff} ., 650 \mathrm{ff} ., 656,658$

Eisner, Kurt 21, 25, 75

Ellerhusen, Paul $375 \mathrm{f}$.

Eltz v. Rübenach, Paul Frhr. 62,80

Emminger, Erich 28, 31, 35

Endres, Fritz 16, 231

Engel, Arnold 240, 273, 1212

Engel, Fritz-Karl $348 \mathrm{ff} ., 352 \mathrm{f}$.

Engert, Karl $232 \mathrm{ff} ., 239,245,258,678,969 \mathrm{f}$.

Epp, Franz Xaver Ritter v. 230, $232 \mathrm{ff} ., 239$, 323,332 ff., 380,382 f., 393, 395, 397, 399 , $408 \mathrm{ff}$., 428, $435 \mathrm{f}$., 438, 441, 447, 449, $550 \mathrm{f}$., 586,638 ff., $642,644,722,834$

Ernst, Karl 344, 442, 458, 480

Erzberger, Matthias 56

Eschenburg, Theodor $445 \mathrm{f}$.

Esser, Hermann 395 f., 638

Fabig, Paul 16, $192 \mathrm{ff}$.

Fabricius, Hans 133

Faulhaber, Michael v. 383, 513

Fechenbach, Felix 25 ff., 38, 47

Felmy, Hellmuth 963

Ficker, Hans 260

Fink, Gustav 352f., 364, 377

Fischer, Ludwig 234, 875

Fliess, Julius 70, 184

Florian, Friedrich Karl $356 \mathrm{ff} ., 965$

Förster, Walter 460, 464, 480

Fraenkel, Ernst 470,567

Frank, Hans $10,57,64,68,70,81 \mathrm{ff}$, $86 \mathrm{ff}$., 93, 95 f., 99, 102, 104f., 110, 115, 122, $128 \mathrm{ff} ., 132 \mathrm{f} ., 136,139,141,143 \mathrm{ff}$., 150 , $154,163,221,230$ ff., $261,265,274,276$, 282, 285, 323, 325, 332 ff., 336, 343, $380 \mathrm{ff}$., 386 ff., 397 ff., 406 f., 409 ff., 427, 429, 434 ff.,
$439,449,473,477,546,548,568,572$ ff., 586 f., 638 f., 641 ff., 645, 694, 698, 707, 747, 752 ff., 755 ff., 767 ff., 773,791 ff., 798 f., 802 ff., 807,810 ff., 817 ff., 834 f., 852,855 , 869,896 f., $927,929,933,938,961,965$ f., $981,984,994,1002$ f., 1006, 1016, 1048 f., $1111,1116,1120,1129,1134,1139$

Franz, Wilhelm $641 \mathrm{ff} ., 647$

Freisler, Roland 64, 71, 79, 87 f., 100 ff., 106, 109 f., 114, 118, 123, 135, 140 ff., 147, $158 \mathrm{ff}$., $174,184 \mathrm{ff}$., 208, $213 \mathrm{ff}$., $219 \mathrm{f}$ f, $223 \mathrm{ff}$., $229,243,246$ ff., $256,261,265,270,272 \mathrm{f}$., $282,300,303,308,312 \mathrm{f} ., 316,330,340$, 344 ff., 352 f., 355 ff., $360,367,379,431$, 446 f., $453,457,462$ ff., 468,472 f., 477 , 489 ff., 505,510 ff., 515 ff., $520,522,524$, 526 f., 530 f., 540, 554, 557, 577, 591, 596, $598 \mathrm{f} ., 603,608 \mathrm{f}$., $611 \mathrm{f} ., 621 \mathrm{f}$., 626,631 , $652,654,660,662,664,666,671$ f., 680, 687, 689, $696 \mathrm{f}$., 701, 723, $728 \mathrm{f}$., 732, $735 \mathrm{ff}$., 739 ff., 745, 754f., 757, 760, 764f., 768, 770 ff., $776,778,780,782,784,789,791$, $793,805,811,831,847,849 \mathrm{f}$., 855 ff., $864 \mathrm{f}$., $867 \mathrm{f} ., 873,879 \mathrm{f}$., 886, 902, $905 \mathrm{ff}$., $909 \mathrm{ff}$., 916 ff., 922 f., $927,933,937,939$ ff., 945 , 951, $955 \mathrm{f} ., 964,966,970,975,978 \mathrm{f} ., 986 \mathrm{f}$., 1008 ff., 1015, 1017 f., 1023, 1028, 1030, 1033 ff., 1069, 1071, 1073, 1076 ff., 1080 ff., $1088 \mathrm{f}$., 1097 ff., 1101 ff., 1105 ff., $1117 \mathrm{f}$., $1123,1130,1133,1139,1145$

Freißler, Dr. (jüdischer Arzt) 666

Frey, Adolf Heinrich 493

Frey, Wilhelm 287, 1212

Frick, Wilhelm 9, 33, 42, 67, 76, 83, 88, $92 \mathrm{ff}$., $105,111,117,123,132 \mathrm{f} ., 135,137,162$, 164, 169, 178, 190 f., 197 ff., 204, 207, 215 f., $268 \mathrm{f}$., $273,322,329,335,340,349,368$, 375,381 f., 401, 406, 423, 425 f., 429, 433, $441,450,456,479$ f., $483,501,509,513$, 535 f., 546 ff., $569,571,575 f$ f., 586, 603 f., 647 ff., 700, 702, 707, 725, 741 f., 820, 823, $826 \mathrm{f} ., 829,832,843,847,864,869 \mathrm{ff} ., 876 \mathrm{f}$., 903 f., 906, 912 f., 953 f., 959, 963, 984, 1017, $1050,1082,1119,1124 \mathrm{f}$.

Friedrich, Kurt 261, 309

Friedrich, Wilhelm 261

Fritsch, Werner Frhr. v. 76, 256

Fritzsche, Hans 484

Froböß, Hellmut 280, 1214

Fuchs, Georg 37

Fuchs, Hans 384, 396, 400, $404 \mathrm{f}$.

Funk, Walther 454, 820, 912

Gabriel, Helmuth 287, 1214

Galen, Clemens August Graf v. 519, 521

Gans, Erich 440, 464 
Gargas, Sigismund 25, 28

Garßen, Adolf v. 225, 281, 1211

Gast, Peter 521

Gayl, Wilhelm v. $58 \mathrm{ff}$.

Gentz, Dr. (Ministerialrat im preußischen Justizministerium) $223 \mathrm{f}$.

Gerber, Alexander 236,1213

Gerland, Heinrich 764

Gerlich, Fritz 440

Geßler, Otto 32

Giese (erpresserischer Kindesräuber) 894, 896

Gildisch, Kurt 442

Gillen (Oberjustizrat im preußischen Justizministerium) 223

Gipser, Franz 232, 239

Gisevius, Hans Bernd 456, 552, 555

Glaser, Alexander 440

Gleispach, Wenzel Graf $311,498,754,760$, $765,786,790,863 \mathrm{f} ., 1015$

Globke, Hans 879

Goebbels, Joseph 350, 421, 435, 438, 484 ff., $488,665,750,769,800,902,904,964$

Goedel, Hans 862, 1012

Göhring, Ludwig 391

Goerdeler, Anneliese 76, $81 \mathrm{f}$.

Goerdeler, Carl 76, $81 \mathrm{f}$.

Göring, Hermann 9, 76, 83, 103, 120, $122 \mathrm{f}$., $132,147,157,161,164,221 \mathrm{ff} ., 233,284$, $320 \mathrm{ff} ., 324 \mathrm{f}$., $329 \mathrm{ff}$., 334, 342, 346, 348 , $350 \mathrm{ff}$., $357,363 \mathrm{ff}$., $421,431,433 \mathrm{f}$., $437 \mathrm{f}$., 442,445 ff., $454,457 \mathrm{ff}$., $462,468,473 \mathrm{ff}$., 485, 488 f., 491 f., 509,535 f., 547 f., 552 ff., $557,559,568,570$ f., 597, 646, 649, 660, $668,675,678,689,691,695,719$ ff., 726 , $750,799,801$ f., 818,820 ff., 831 ff., 847 , 854, 886, $903 \mathrm{f}$., $906 \mathrm{f}$., $913,922 \mathrm{f} ., 957,959$, $964,1082,1111,1120$

Goetsch, Rudolf 273, 1214

Götz, Josef 634

Götze, Max 806, 897 f.

Götze, Walter 806, 897

Goldenberger, Franz Xaver 56

Goldmann, Ernst 634

Goldschmidt, Gustav 223

Goldschmidt, James 981

Goltz, Rüdiger Graf v. der 71, 76, 81, 349f., 754, 769, $772 \mathrm{f} ., 783,1011,1015,1033 \mathrm{f}$., $1037,1045 \mathrm{f}$.

Gorgass (Gorgaß), Hans Bodo 526

Gottheiner, Georg $60 \mathrm{f}$.

Gramm, Hans 688

Graßmann (Generalstaatsanwalt in Danzig) 287,1211

Grau, Fritz 259, 754, 765, 768, 797, 866

Grau, Richard 452
Grauert, Ludwig $161,328,330,349,359$, $363,456,695,699,703,721$

Greinz, Hermann 278, 1212

Greiser, Arthur 280

Grimm, Wilhelm 357f., 863

Grohé, Josef 229

Grossmann, Hermann 127, 165

Groten, Theodor 363

Grünspan, Herschel 484, 487

Grußendorf, Werner 71, 79, 259

Günder, Bruno 57

Guérard, Theodor v. 253

Gürtner, Anna 13

Gürtner, Franz jun. 21, 70, 74

Gürtner, Fritz 11, 21, 70, 74, 82, 447, $454 \mathrm{f}$.

Gürtner, Heinz 21, 74

Gürtner, Luise 11, 21, 73f., 82, 447

Gütt, Arthur $500 \mathrm{f}$.

Gutjahr, Ernst 226, 1211

Haacke, Werner v. 224, $346 \mathrm{ff} ., 367,369$, $443 \mathrm{ff}$., 457, 462, 464, 492, 654, 715, 717, $760,954 \mathrm{f}$.

Haastert, Johannes 106, 211, 246, 265

Hachenburg, Max 20

Häbich, Walter 440, 464

Hafner, Karl 240, 1212

Hagemann, Franz 284, 958, $1212 \mathrm{f}$.

Hahn, Hermann $282 \mathrm{f}$., 526, $1213 \mathrm{f}$.

Handschuch, Hugo $392,640,642 \mathrm{ff}$.

Haniel v. Haimhausen, Edgar 34

Hansen, Heinrich 226,604

Hansen, Peter 1213

Hardt, Otto 1213

Harmening, Rudolf 81,243

Hartl, Albert 311

Hartung, Friedrich (Fritz) 1012

Hartung, Hans 22

Hartwig, Dr. (Ministerialdirektor im preußischen Justizministerium) $222 \mathrm{f}$.

Hattenhauer, Hans 2, 7

Hauptvogel, Fritz 244, 258

Hausmann, Leonhardt 635,637

Hayn, Hans 437, 458

Hefelmann, Hans 499 ff., 508 ff., 533

Heim, Heinrich 797

Heinen, Johann 677

Heines, Edmund $124 \mathrm{f} ., 326,328,437,458 \mathrm{f}$, 468

Heines, Oskar 459

Heintzeler, Karl 240, 284, 1214

Heintzeler, Wolfgang 74, 81, 245

Heinze, Rudolf 27

Held, Heinrich 17, 25, 28, 44, $46 \mathrm{ff} ., 51 \mathrm{ff}$, $56 \mathrm{f} ., 68,231$ 
Helldorf, Wolf-Heinrich Graf v. 443 f., 709

Hellmuth, Otto 380

Hempen, Bernhard 225, 1212

Henkel, Heinrich 765, 769, 1035

Henlein, Konrad 278

Hepke, Adolf 226, 1213

Hereth, Adam 440, 464

Herrmann, Franz $222 \mathrm{f}$.

Herrmann, Philipp 39, 42

Herwegen, August 1211

Herwig, Paul 244

Heß, Erwin 240, 273, 1214

Heß, Rudolf $33,46,88,178 \mathrm{f} ., 187,204 \mathrm{f}$., 207, 213, 217 f., 255, 257 f., 260, 262, 264, 273, 277, 281, 329, 356, 370 f., 421, 423, $425,433,436,478$ f., 489 f., $748,750,753$, 756 f., 767, 769, 792 ff., 799, 803, 807 ff., 812 ff., 833, 853, 876 f., 885, 903 f., 922, 962, ff., $970,1011,1015,1045$ ff., 1124

Hesse, William 244

Hessen, Christoph Prinz v. 352

Heuber, Wilhelm 141

Heusinger, Bruno 240, 271, 1211

Heuwieser, Albert 236, 276 f., 1211

Heyde, Werner 499, 503 f., 509 f., 524, 527 ff., 533

Heydebreck, Hans Peter v. 437,458

Heydrich, Reinhard 76, $82 \mathrm{f} ., 236,345,353$, 380,390 f., $394,406 \mathrm{ff} ., 434,438,440,442$, 448f., 456f., 479, 485, 487, 489, 491, 509, $532,544,548,550$ f., $555,559,561,566$, 574 ff., $578,585,595,597$ f., 605 ff., 612 , $618 \mathrm{f} ., 622,628 \mathrm{ff} ., 639,643,645,659$, 676 ff., 681, 684, 686, 699 f., 703, 705, 707, 710 ff., $718,874,879,884,888$ ff., 905,994 , $1080,1097,1110$

Heydte, Friedrich-August von der 452

Hildebrandt, Friedrich $272 \mathrm{f}$.

Hildebrandt, Karl 216, 304, 306f., 310

Hilfrich, Antonius 522

Hilgenfeldt, Erich $797 \mathrm{f}$.

Hilgers, Alfred $354 \mathrm{f}$ f, $357 \mathrm{f}$., 362

Hilpert, Hans 24, $52 \mathrm{ff}$.

Himmler, Heinrich $3,76,78,82 \mathrm{f} ., 233,345$, $353,362,364$ ff., 369,377 f., 380 f., 387,389 , 394, 401, 405 ff., $410,412,433 \mathrm{f}$., 437 , 439 ff., 444 f., 448,450 ff., 456 f., 459 ff., 468 ff., 478 f., 485 f., 532 f., 546,550 ff., 557 ff., 562 ff., $566,568,570$ f., 573,576 , 591, 597 f., 601, 607, 618, 621, 634, 637 ff., 645,649 ff., 658 f., 661 ff., 665,672 ff., 683 , 688 ff., 697, 699, 702 f., 705 ff., 710 f., 716 , 723 ff., 727, 738, 741 f., 745, 750, 796, 801, $806,812,848,884$ ff., $888,917,1003,1012$, 1017,1020 f., $1030,1081,1122,1124 \mathrm{f}$., 1127
Hindenburg, Paul v. 9 f., 57 ff., 62 ff., 66, 68, 75,88 ff., 93 f., 133 f., 146, 148, 177, 323, $326,334 f ., 345,393,449,454,465,535$, 826,829 f., 946 f., 1050, 1114, 1116, 1120 , 1128

Hippel, Fritz v. $\quad 1,828$

Hirschberg, Max 28

Hitler, Adolf $9 \mathrm{f}$., $28 \mathrm{ff} ., 33 \mathrm{ff} ., 39,41 \mathrm{ff}$., $51 \mathrm{f}$., 59, 61 ff., 72,77 ff., 82 f., 87, 89 f., 94, 97 f., $100 \mathrm{ff} ., 109,111,115,117,119,121$ ff., 126 , $130 \mathrm{f} ., 133$ ff., 139, 141, 145 f., 158 f., 164, 169 f., 173, 176, 178 f., 187, 191 ff., 206 ff., 212 ff:, 217, 219, 221 f., 232, 235f., 251, 255, 257 f., 260, 275, 277, 290, 292, 319, 322 f., $325,327,329$ f., 334 f., 338, 340, 343, 360, 362,365 ff., 369,372 ff., $378,380,390,392$, $398 \mathrm{f}$., 402, 407, 413, $416 \mathrm{f}$., 419, $421 \mathrm{ff}$., $431 \mathrm{ff}$., $441 \mathrm{f}$., $448 \mathrm{ff}$., $462,464,468 \mathrm{ff}$., 472 , 475 f., 478 f., 486 ff., 491 f., 496,499 ff., $507 \mathrm{ff}$., $512 \mathrm{f}$., $521,535,546,550,553,558 \mathrm{f}$., 570 f., $573,597,605$ ff., $609,617,627$ ff., $643 \mathrm{f}$., 646, 649, 654, 656, 664f., $668 \mathrm{f} ., 671$, 675 ff., 686 ff., 694,706 ff., $712,725,738$, $741,745,747$ ff., 756 ff., $769,792,794$ ff., 798 ff., 819 ff., 824, 826, 829 f., 833, 835 ff., $846 \mathrm{f}$., 868,875 ff., 881 f., 884, 892, 894 ff., 903 f., 910 ff., $915,920,922$ ff., 928,955 f., 958 ff., 968 ff., $973,1007,1011,1026,1038$, $1040,1047 \mathrm{ff}$., $1052,1055,1071 \mathrm{ff} ., 1080 \mathrm{f}$., 1090, 1095, 1106, 1110, 1113, $1115 \mathrm{ff}$., 1128 ff., 1132, 1134 ff., 1138 ff., 1146

Hoberg und Buchwald, Anton Frhr. v. 483

Hoche, Alfred 497

Hoche, Werner $61,417,425,1017,1021$

Hock, Karl Ritter v. 215

Höfle, Anton 1059

Högl, Eduard 279, 1213

Hoegner, Wilhelm 29f., 34, 47, 75

Höhler, Ali 324, 675

Höhn, Reinhard 994, 1139

Hoeller, Karl 81

Hölscher, Heinrich 125, 132, 222 f., 225, 281 , $288,323,325,529,1211$

Höß, Rudolf 677

Hofer, Franz 278, 286

Hoffmann, Joachim 349 ff., 364, 372, 377

Hoffmann, Johannes 16,18

Hoffmann, Richard 229

Hofmann, Hans Georg 964

Hohmann, Wilhelm 226, 1212

Holz, Karl 274

Holzbauer, Andreas 246

Holzhäuer (Oberstaatsanwalt am Oberlandesgericht Stuttgart) 507, $510 \mathrm{f.}, 526$

Holzschuher, Wilhelm Frhr. v. 355

Hoos, Fritz 240, 1211 
Huber, Hans 246, 261

Hueber, Franz 174 f., 184, 260, 268, 581, 596, $807,1026 \mathrm{f}$.

Hülle, Werner 1017

Hüttner, Alfred 239, 279, 1212

Hugenberg, Alfred 9, 52, 55, 68, 322

Huther (Senatspräsident am Oberlandesgericht Rostock) 272

Hutzelmann, Wilhelm 641

Jacobi, Erwin 61

Jacobs, Erwin (Teltower Sparkassenräuber) $679 \mathrm{ff} ., 911 \mathrm{f}$.

Jäger, August 1002

Jähnichen, Rudolf $368 \mathrm{ff} ., 373 \mathrm{f}$.

Janßen, Wilhelm 226, 1213

Jockwer (Oberlandesgerichtspräsident in

Hamm) 225

Joël, Curt 57, 71, $241 \mathrm{f} ., 253$

Joël, Günther 224, $347 \mathrm{f}$ f, $354 \mathrm{ff}$., 363, $366 \mathrm{f}$., $453,457,462 \mathrm{ff} ., 472,486,489 \mathrm{f}$., 493,533 , 653 ff., 683 f., $712,715,1080$

Johe, Werner 2

Jonas, Martin 243, 258

Jordan, Rudolf 213

Jorns, Paul 960, 969, 971, 1100

Jüttner, Hans 369, 532

Jung, Edgar 442, 449, 456, $479 \mathrm{f}$.

Jung, Friedrich 281, 529, 1211

Jung, Heinrich 282, 1212

Jung, Philip Wilhelm 240

Kaas, Ludwig 9

Kahl, Otto 237, 1211

Kahn, Arthur 634

Kahr, Gustav Ritter v. 20 ff., 30, 32 ff., 40, 440, 456, 459

Kaindl, August 464

Kallenbach, Richard 232, 239

Kamphausen (Stadtbaurat in Waldenburg) 455, 461, $464 \mathrm{ff} ., 1122$

Karge, Max 275, 1213

Karpenstein, Wilhelm 226, 350

Karpf, Eugen 19

Katz, Delwin $641 \mathrm{ff} ., 647$

Katzenberger, Leo 909

Kaufmann, Karl 274, $374 \mathrm{ff} ., 488,669,672$, 705

Kaul, Friedrich Karl 2

Kaulbach, Günter $58,379,758,881$

Keitel, Wilhelm 817, 820, 1082

Kelsen, Hans 452

Kempner, Robert 506

Kerrl, Hanns 64, 87 f., 91, 95, 99 ff., 109 f., 122,127 ff., 133, 135 ff., 139 ff., 153 f., 156 f., 160, 163, 189, 214, 218 f., 221 ff., 226 ff.,
231, 243, 261, 275, 283 f., 299, 301 ff., 308, 315,323 f., 327 f., 330 ff., 339 f., 343 f., 346 , 497, 587, 610, 675, 721, 753f., 757, 759f., $765,767,776,794,803,807,819,831,864$, $1054,1056,1116 \mathrm{f}$.

Kiesow, Wilhelm 243

Kiesselbach, Wilhelm 240,1212

Killinger, Manfred Frhr. v. 369

Killy, Leo 203

Kinder, Elisabeth 7

Kirchner, Harald 7

Kirchner, Hildebert 7

Kisch, Wilhelm 766

Kißkalt (Rechtsanwalt in München) 12

Klässel, Oscar 106, 224, 244, 247, 258

Klaiber (Polizeipräsident in Stuttgart) 1017, $1021 \mathrm{ff}, 1027 \mathrm{ff}$.

Klauer, Georg 243

Klausener, Erich $442,445,447,457,480$, 483, 698

Klausener, Hedwig 482

Klee, Friedrich 246, 760, 765

Klee, Karl 754, 857

Klemenz, Karl 261

Klemm, Herbert 246, 261, 496, 544, 561, 594, 619, 624 ff., 718

Klinzmann, Wilhelm 1081

Knapp, Klaus $246 \mathrm{f}$.

Knilling, Eugen v. 25, 27, 29, 33, 35, $41 \mathrm{f}$.

Koch, Erich 242, 275, 283 f., 287

Koch, Paul 240, 1211

Koch(-Weser), Erich 253

Koehler, Karl 244

Koehne, Waldemar 248

Köllinger, Anton 286, 1213

König, Heinrich 438

Königsberger (Reichsgerichtsrat) 248

Köppel, Ewald 461, 479

Körner, Paul 157, 353, 456, 922

Kötter, Dr. R. (Hauptschriftleiter im Fränkischen Kurier) 69, 72

Koffka, Johannes 243

Kohlrausch, Eduard $73,754,760,765,772$, $789,851,856,860,866,1015,1033$ ff., $1041 \mathrm{ff} ., 1071$

Kolbe, Dieter 2

Kollat, Paul 244

Kompalla, Ludwig 910, 1071 ff., 1078

Kompalla, Paul 1071, $1073 \mathrm{ff}$.

Kraell, Alexander 17,71

Kramberg, Paul 286f., 1213

Krapp (Landgerichtspräsident in Bamberg) 237

Krauss, Günther 310

Kraußer, Fritz Ritter v. 399

Krautmann, Edmund 278, 1213 
Kreyßig, Lothar $165,202,505,508,511 \mathrm{f}$, 515

Kriebel, Hermann $\quad 28,31 \mathrm{ff}$, 39, $44 \mathrm{ff}$.

Kriege, Walter 71, 244, 265

Krieger, Karl 244

Krinke (Generalstaatsanwalt in Breslau) 226, 1211

Kritzinger, Wilhelm 176, 179, 187, $196 \mathrm{f}$, 199f., 203, 243, 259, 277, 506, 555, $557 \mathrm{f}$., $570,590,608,679,687,748,806,818$, 821 f., 903 f., 906, 913, 922, 924

Krug, Karl 261, 346f., 472, 760, 958, 965, 1095

Kube, Wilhelm 222, 226, 662

Kühn (Oberregierungsrat im Reichsjustizministerium) 244, 246

Kühnemann, Herbert 261

Küstner, Otto 273, 1214

Kuhnt, Gottfried 225, 1213

Kulenkamp, Richard 1214

Kunisch, Siegmund 244, 246

Kunze (Obertruppführer im Referendarlager „Hanns Kerrl“) 306

Kupfer, Angelus 74

Kutzner, Helmuth 246, 258

Lammers, Hans Heinrich 94, $100 \mathrm{ff} ., 109 \mathrm{ff}$., $126,135,176,178$ f., 187,192 ff., $217,257 \mathrm{f}$., 260, $275 \mathrm{ff} ., 352,421,429,431 \mathrm{f} ., 447 \mathrm{f}$., 450 f., 454, 457, 462, 499, 501 f., 507 f., 510 , $513,521,523,533,559,598,652,665,675$, 678 ff., 683, 687 f., 748 ff., 756 f., 760,793 ff., 802,805 f., $810 \mathrm{ff},, 816 \mathrm{ff}$, $820 \mathrm{ff}$., $827, \mathbf{8 8 5}$, 895, 897, 902, 904, 906, 910, $912 \mathrm{f}$., $922 \mathrm{ff}$., 959, 962 f., 966, 972, 994, 1038, 1048 f., 1055, 1073 f., 1076, 1078, 1141

Landfried, Friedrich Walter 132

Lang, Franz 240, 1212

Langhoff, Wolfgang 363

Lasch, Karl 758

Latacz, Paul (Teltower Sparkassenräuber) $679 \mathrm{ff}, 911 \mathrm{f}$.

Lauterbach, Kurt 246

Lautz, Ernst 79, 283, 287, 602, 971, 1002, $1015,1024,1033 \mathrm{f} ., 1211,1212$

Lawall, Erich 306, 311

Lechner, Oskar 246

Lehmann, Julius Friedrich 33

Lehmann, Rudolf $243,259,367,821,942$, $981,994,1016,1021,1033 \mathrm{f} ., 1046,1065$, 1073

Lehrburger, Karl 635

Leimer, Karl 285, 754, 1213

Lembke, Karl Heinz 25, 28

Lent, Friedrich 50

Lentz, Baptist 244
Leoprechting, Hubert Frhr. v. 38

Lerchenfeld, Hugo Graf $22 \mathrm{ff}$.

Letz, Rudolf 114, 192, 211, 311, 573, $623 \mathrm{f}$., 974

Leuchs, Edwin 237, 282, 1213

Levi, Paul 22

Leviné, Eugen $18 \mathrm{f}$.

Ley, Robert 433, 570

Leyendecker, August 38

Lichtenberg, Bernhard 522

Lichtenberger, Gustav 1015

Liebermann von Sonnenberg, Erich 724

Liebmann, Kurt 66

Liese (Justizinspektor) 1002

Lindbergh, Charles 894, 896

Lindemann (Ministerialdirektor im preußischen Justizministerium) 223

Lindemann, Erich 461, 463, 478, 480, 482

Lindemann, Johanna 461,482

Linden, Herbert 503, 506, 508

Lippert, Michael 435, 437, 446, 644

Liszt, Franz v. 790

Litten, Hans 74

Lösener, Bernhard $866,868,870$

Lohse, Hinrich 156f., 226, 228 f., 286, 338, 340

Lorenz (Landgerichtsdirektor in Leipzig) 754

Lorenzen, Sievert 166, 170

Loritz, Hans 364

Lossow, Otto Hermann v. $32 \mathrm{f} ., 35,40$

Lubbe, Marinus van der 535, $826 \mathrm{f} ., 829 \mathrm{f}$., $834 \mathrm{f} ., 894,957 \mathrm{f}$.

Ludendorff, Erich 33, 36, 39

Lüdtke, Egon 688

Lümkemann, Irma $71,73 \mathrm{f}$.

Luetgebrune, Walter 228, 403, 413, $417 \mathrm{ff}$., 423, 426 ff., 457,766

Luftglass, Markus 688

Lutter, Kurt 824

Lutterloh, Ernst 244, 246f., 260

Lutze, Viktor $356,359,364,372,396,431 \mathrm{f}$., 472, 963

Machhaus, Hugo 37

Mackensen, August v. 177, 349

Majer, Diemut $288 \mathrm{f}$.

Malsen-Ponickau, Johann-Erasmus Frhr. v. 387

Malzan, Karl 245, 271

Manglkammer, Friedrich 237,1213

Martin, Benno $387 \mathrm{f}$., $390 \mathrm{f}$.

Martin, Karl 942, 1015, 1026, 1028, 1035, 1213

Marx, Rudolf 244, 246f.

Marx, Wilhelm 35 
Maßfeller, Franz 869

Mayer, Hellmuth $766,852,862$

Mayr, Franz 413

McCloy, John 287

Medicus, Franz Albrecht 105

Meiser, Hans 69, 83

Meisinger, Josef 444, $480 \mathrm{ff} ., 489,711$

Meißner, Johannes 285, 514f., 526, 1212

Meißner (Meissner) Otto 62, 360, 362, 373, $450,454,469,605,686 \mathrm{f}$., 708, 826, 829, 846, 1071

Meldt, Fritz 278, 1212

Mergenthaler, Christian 147

Merth, Hermann 261

Mettgenberg, Wolfgang 243, 264, 417, 431

Meukel, Leonhard 99, 232, 239, $435 \mathrm{f}$.

Meyer, Alfred 282

Meyer, Karl 23 f., 30, 34

Meyer-Quade, Joachim $341 \mathrm{f}$.

Mezger, Edmund 754, $764 \mathrm{f}$.

Minde, Hugo 225, 284, 1213

Mitzschke, Gustav 261

Moehrs, Walter 225, 1213

Möller (Oberregierungsrat bei der Gestapo Berlin) $715 \mathrm{f}$.

Mößmer, Ferdinand 869

Morsey, Rudolf 7

Moser, Johann 285f., 1212

Moser v. Filseck, Carl 17, 35, 47, 54, $56 \mathrm{f}$.

Mosler, Gotthard 1214

Mühsam, Erich 19

Müller, Dr. (Oberregierungsrat, SS-Standartenführer) 489

Müller, Adolf 240, 271, 1211

Müller, Emil 246

Müller, Gustav 237

Müller, Heinrich (Generalstaatsanwalt in Braunschweig) 240,1211

Müller, Heinrich (Leiter der Ministerialabteilung Justiz im hessischen Staatsministerium) 240

Müller, Heinrich (Leiter der Politischen Polizei) 627f., 631, 683, 713, $717 \mathrm{f}$.

Müller, Josef 10

Müller, Karl Alexander v. $11 \mathrm{f}$.

Müller-Meiningen, Ernst 19f., 28

Mutschmann, Martin 165, 279, 282, 368 ff., 1111

Nadler, Max 106, 141, 195, 203, 219, 223 , 244, 247, 263, 265, 281, 298, 446, 699

Nagler, Johannes $417,754,765,826 \mathrm{f}$.

Nebe, Arthur 724, 1017, 1021

Nebelung, Günther 271, 1211

Neef, Hermann $87 \mathrm{f}$.

Nefzger, Sebastian $636 \mathrm{f}$.
Neithardt, Georg 37 ff., 44, 236, 274, 1213

Neubert, Reinhard 136, 176 f., 183 f., 189, $572,942,1015,1041$

Neumann, Erich 557, 903

Neumann, Richard 165, 248

Neumayer, Edmund 437

Neurath, Konstantin Frhr. v. 59, 280, 483, 824, 835, 876

Nieberding, Arnold 84

Niemöller, Else 78

Niemöller, Martin 74, 76 ff., 80, 599

Niethammer, Emil 68, 71 f., 74, 769, 772, 942, 1012, 1015, 1024, 1033 f., 1036, 1038 ff., 1062, 1098

Nitsche, Hermann Paul 524

Noack, Erwin $176 \mathrm{ff} ., 766$

Nörr, Siegmund 70 f., 73 f., 81, 261

Novelli, Giovanni 70

Oberg, Karl Albrecht 378

Obernitz, Hanns-Günther v. $387,389 \mathrm{ff}$, 404

Oetker, Friedrich $\quad 427,766,826$ f., 981, 1016

Ohlendorf, Otto 683

Ohnesorge, Wilhelm 849

Olschewski, Wilhelm 19

Olshausen, Justus v. 499

Osburg, Hugo 244

Oswald, Karl $44 \mathrm{f}$.

Pätzold, Erwin 243, 246

Palandt, Otto 106, 219, 224f., 244, 303, 307

Papen, Franz v. 9 f., 49, 57 ff., 68, 132, 222,

$323,326,335,442 \mathrm{f}$., $448 \mathrm{f}$., 455, 458, 479,

$829,946,1003,1119$

Parey, Friedrich 283 f., 331, 596 f., 935, 971, 981, 1019, 1211

Parrisius, Heinrich 969

Paulick, Dr. (Landgerichtsdirektor in Stettin und Gaurechtsberater der NSDAP) 215, 227,281

Pauly, Julius 239

Perk, Willi $363 \mathrm{f}$.

Perlitius, Ludwig 9

Pernet, Heinz 33, 42

Pestalozza, Josef Graf v. 34, 36

Peters, Karl 1056

Pfeiffer, Hans 355, 358

Pflaumer, Oskar $386 \mathrm{f}$., $389 \mathrm{ff}$., 405

Pfordten, Theodor von der 12, 21, 28

Pfundtner, Hans 190, 194, 216, 255, 430, 876, 885, 959, 1012

Pichinot, Hans-Rainer 2

Pleines, Fritz 352, 364, 377

Pöhner, Ernst 22, 28, 33, 39, 44

Pohl, Oswald 654,745

Pohle, Rudolf 72, 178 
Popitz, Johannes 75, 132, 147, 160 f., 826, 843, 872

Popoff, Biagoi 957

Porath, Hans-Joachim 246

Posse, Hans 802

Preger, Konrad Ritter v. $26 \mathrm{f}$.

Preuß, Richard 223

Preußen, August Wilhelm Prinz v. (,Auwi“) 970

Pritzsch, Erich 244

Pünder, Hermann 60

Pünder, Werner 442, 480, 482 f., 698

Püschel, Wilhelm 1

Pufpaff, Walter 1214

Quassowski, Leo 243, 258, 262, 264, 1014

Radbruch, Gustav 451, 765, 780

Raeke, Walter 282, 307 f., 567 ff., 938, 1002, 1008

Raestrup, Johannes 1012

Rasmussen (Fall) 72

Rath, Ernst vom 484 f., 493

Rattenhuber, Johann 394

Reh, Robert 461

Rehn, Fritz $962 \mathrm{ff}$.

Reichau, Rudolf 244, 265

Reichenau, Walther v. 450

Reifner, Udo 3

Reimer, Werner 754

Reinach, Dr. (jüdischer Arzt) 71

Reinecke, Günther 654

Reinle, Heinrich 147, 213, 275, 1212

Reitter, Ekkehard 2

Remmert, Heinrich $363 \mathrm{ff}$.

Rempe, Heinrich 395

Renthe-Fink, Cécil v. 73, 81

Resch, Alfred 234, 239

Reuthe, Kurt 279, 1213

Richter, Hans 243, 258

Richter, Heinrich 246

Richter, Reinhold 16, 106, 242, 244, 248, $251,258,431 \mathrm{f}$.

Riese, Otto 243, 246, 261

Rietzsch, Otto 244, 604, 743, 760, 765, 797, $802,818,821,899$

Rilk, Otto $1002 \mathrm{f}$.

Ritterbusch, Paul 1139

Röhm, Ernst 33, 42 f., 321, 329, 331, 343, $345,354,369,380,382,389 \mathrm{f} ., 396 \mathrm{ff}$., 412 ff., 419, 421,424 ff., 433 ff., 437 f., 441 , $445,448,450,454 \mathrm{f}$., $457 \mathrm{f}$., $471,552,643 \mathrm{f}$., $654,959,963$ f., $1120 \mathrm{ff}$.

Röhrbein, Paul 440

Röpcke, Willy 240, 1211

Röver, Carl 279, 362, $364 \mathrm{f}$.
Romeiß, Hans $309 \mathrm{f}$.

Rosenberg, Alfred 864

Rosenfeld, Siegfried 224

Roth, Dr. (Landgerichtsdirektor in Dresden) 370

Roth, Christian 18, $21 \mathrm{f}$.

Rothenberger, Curt 71,107,112, 114, 143, 170, 240, 274, 304, 309 ff., 317, 374 f., $377,379,488,530$ f., $563,573,602,612$, $623 \mathrm{ff}$., $667,669 \mathrm{ff} ., 674,705,712,727,733$, 949, 974, 1105 ff., 1212

Rottleuthner, Hubert 3

Rüthers, Bernd 2

Rumschöttel, Hermann 7

Rupp, Johannes Ludwig 327

Ruppert, Kuno 195, 245

Rust, Bernhard 1002

Rutz, Ottmar 49

Sack, Karl 1015

Saemisch, Friedrich 829

Sahm, Heinrich 76

Salis, Karl 352

Salje, Peter 3

Salm, Elisabeth 578

Saß, Erich und Franz 720

Sattelmacher, Paul 224f., 1213

Sauber, Fritz 19

Sauckel, Fritz 109, 121, 207

Sauer, Franz 106, 243, 247, 265

Sauer, Viktor 228, 286, 1213

Sauerbruch, Ernst Ferdinand 506

Sauerländer, Johann David 232

Sauthoff, Friedrich 244

Sautter, Reinhold 510f., 522

Schacht, Hjaimar 554, 556, 872

Schade, Hermann Frhr. v. 377 f., 958

Schäfer, Ernst $106,242,244,247,261,325$, $416,754,771,793,806 \mathrm{f} ., 812,834,851 \mathrm{f}$., 858, 882, $923 \mathrm{f}$., 931, 935, 940, 981, 994, 1015,1022 ff., 1032, 1037 f., 1042, 1044

Schäfer, Karl 861

Schäfer, Leopold 243, 245 ff., 853, 887 f., 917

Schäfer, Werner 364

Schäffer, Fritz $40,53,58$

Schaeffer, Walter 1211

Schätzl, Martin 438

Schaffstein, Friedrich $765,769,772,790,858$

Schafheutle, Josef $933,935 \mathrm{ff} ., 981,984$

Schallermeier, Luitpold $485 \mathrm{f}$.

Scharf, Friedrich 239

Scharfe, Paul 378, 654

Schattenfroh, Max 73, $81 \mathrm{f}$.

Schaub, Julius 683 f., 686 ff., 911,1080

Schemm, Hans 380,638

Scheuner, Ulrich 452 
Scheurien, Karl 1012

Schiele, Fritz 532

Schieweck, Erich 437

Schiffer, Eugen 981

Schlaich, Ludwig 513

Schlegel, Rudolf 1212

Schlegelberger, Franz $68,70,72,74,81,83$, $86,88,92,102 \mathrm{ff} ., 106,118,120,125 \mathrm{ff}$., $130 \mathrm{ff}$., $135 \mathrm{ff}$., $174 \mathrm{ff}$., 216, $219,241 \mathrm{f}$., 245 , $247 \mathrm{ff}$., 252, 255, 264, 276f., 282, 288, 325 ff., $330,413 \mathrm{f}$., $416 \mathrm{ff}$., 431, 446, 452, 455 , $479 \mathrm{ff}$., 501, 505, 510, $520 \mathrm{ff}$., 527, $529 \mathrm{f}$., 554 , $557,674,680,686 \mathrm{ff}$., $693 \mathrm{f}$., $723,748,754$, 756, 758 ff., 824, 826 ff., 878, 895, 910 , 924, 927 ff., 945 f., 956, 959, 962, 994, $1002,1071 \mathrm{ff} ., 1078,1116 \mathrm{f}$., 1123, 1127, 1141

Schleicher, Elisabeth v. 443,445

Schleicher, Kurt v. $10,58,60,62 \mathrm{f}$., $442 \mathrm{ff}$., 449 f., 455, 458, 946, 1003, 1113

Schlitt, Ewald 1078

Schlögl, Alois $382 \mathrm{f}$., 405

Schloß, Louis 635, $637 \mathrm{f}$.

Schmauser, Ernst-Heinrich 437

Schmid, Jonathan 240

Schmid, Wilhelm 400, 437

Schmid, Dr. Wilhelm (Willi) 440, 464, 479

Schmidt, Eberhard 765

Schmidt, Edgar 244

Schmidt, Hermann 225

Schmidt, Paul 246, 259

Schmidt, Willi 246

Schmitt, Carl 61, 453f., 980, 994 ff., 1018

Schmitt, Hermann 21,34, 55

Schmitt, Kurt 73, 81, 94

Schmitz, Hermann $1012 \mathrm{f}$.

Schmölder, Karl 243

Schmoller, Eugen 240, 1214

Schneidenbach, Hans 754

Schneider, Rudolf 225, 1212

Schneidewin, Karl 1012

Schneidhuber, August 392, 405 f., 436 f., 480

Schneller, Otto 106, 224, 243 f., 247, 258, 265

Schnoering, Karl 284, $1211 \mathrm{f}$.

Schober, Friedrich 277, 288, 1214

Schoene, Heinrich $338 \mathrm{ff}$., 342, 413

Schoetensack, August 427, 766, 1016

Schoetensack, Hermann 260

Schollen, Franz 225, 1212

Schorlemer, Karl Friedrich Frhr. v. 432

Schorn, Hubert 1

Schraut, Rudolf $141,143,234,757,760,933$, 981

Schrems, Prof. Dr. (Domkapellmeister) 74

Schroer, Hermann 356, 358 f., 965
Schütz, Hans 2, 310

Schultz (Staatsrat in Hamburg) 143, 161

Schultze, Erich $1012 \mathrm{f}$.

Schulz, Heinrich 327

Schulze, Fritz (Fiete) 705

Schwarz, Franz Xaver 219, 233

Schwede-Coburg, Franz 353

Schweighardt, Hans 437

Schweling, Otto Peter 1

Schwerin v. Krosigk, Lutz Graf 15, 59, 94, $111,116 \mathrm{f} ., 137,158,164,178,191,258$, 264f., 450, 455, 483, 734f., 835, 872, 1052

Schweyer, Franz 24, 29 ff., 38 f., 41, 48

Schwinge, Erich 836

Schwister, Wilhelm 224f., 288, 304, 1212

Scriba, Ludwig 272, 1211

Seebens, Melle 283f., 287, 1211

Seeckt, Hans v. 32

Seefeld, Adolf $709 \mathrm{f}$.

Seel, Hans $133 \mathrm{f}$.

Seißer, Hans Ritter v. $32 \mathrm{f}$., 35, 40

Seldte, Franz 9, 94, 833

Sembach, Emil 459, 463

Semler (Geheimrat) $417 \mathrm{f}$.

Semler, Hans 281 f., 1212

Severing, Karl $59 \mathrm{f}$.

Seydel, Helmut 1002

Seyß-Inquart, Arthur 175, 178, 260

Siebert, Ludwig 230, $234 \mathrm{f} ., 333,380 \mathrm{ff}$., $389 \mathrm{f}$., $392 \mathrm{f} ., 395,397 \mathrm{f} ., 406 \mathrm{ff} ., 421,435,441 \mathrm{f}$., $637 \mathrm{f} ., 640 \mathrm{ff}$.

Siebert, Wolfgang 1139

Siegel, Karl 232, 236, 279, 1214

Siegert, Karl 311

Siegfried, Paul 240, 1214

Siehr, Kurt 223

Simmerding, Josef 38,40

Simon, Dieter 1

Sklarek, Max 274

Solmitz, Fritz 375

Sommer, Karl 50

Sommer, Martin 82, 258, 261

Sommer, Therese 82

Sommer, Walther 273, 795, 807, 1045

Sotier, Adolf 236, 285, 1213

Spangenberger, Heinrich 57, 112, 231, 234, 239, 411, 439 f., 639

Sperr, Franz 75

Spieler, Christian $303 \mathrm{f}$.

Sprenger, Jakob 213 f., 245, 259, 271 f., 279

Spreti, Hans Graf v. 437

Sprick, Gustav $232 \mathrm{ff}$.

Springmann, Eduard 962, 965, 969

Stadelmann, Otto 279,1212

Stäcker, Otto 281,1214

Staff, Ilse 2 
Stagel, Friedrich 261

Stahlecker, Walter 696

Stammler, Otto 276,1211

Stampe, Eugen 246

Staud, Eberhard 244

Stauffer, Ferdinand 33

Stegmann, Wilhelm 395

Stein, Hermann 286, 1213

Steinaecker, Walther Freiherr v. 222 f., 274, 282, $1211 \mathrm{f}$.

Steinbrenner, Hans 634,642

Steinmeyer, Hans 74

Stempfle, Bernhard Rudolf 440 f., 459

Stenglein, Ludwig $30 \mathrm{f} ., 36 \mathrm{f} ., 41 \mathrm{ff}$.

Stenzer, Franz 640

Stepp, Walther 235f., 406f., 411, 437, 440, $448,589,639,643,722,958$

Stich, Johann 286, 526, 1214

Stiebel, Martin 644

Stier, Martin 526

Stoffel, Jakob 21

Stoffel, Luise s. Gürtner, Luise

Stolleis, Michael $1 \mathrm{f}$.

Stolzenburg, Friedrich Wilhelm 336

Strasser, Else 480,482

Strasser, Gregor $62 \mathrm{f}$., 442, 445, $457 \mathrm{ff}, 480 \mathrm{ff}$.

Strasser, Otto 41

Strauß, Alfred 635, 637

Strauß, Julius 405

Strauß, Simon 405

Strauß, Walter 529

Streckenbach, Bruno 375, 604f., 623, 712

Streicher, Julius 230f., 274, 285, 380, $394 \mathrm{ff}$., 404, 870

Stresemann, Gustav $30 \mathrm{f}$.

Stritzl, Oskar 278, 1212

Stuckart, Wilhelm 271, 821, 879, 903, 906, 994, 1211

Stürenburg, Ulrich 226,1214

Stützel, Karl 51

Stumpff, Hans-Jürgen 963

Sturm (I. Staatsanwalt in München) 16

Sturm, Reinhold 281, 287, 1211, 1214

Sturm, Siegfried 1017, 1041

Suchomel, Hugo 260 f., 264, 527, 529, 942 , 1017

Surén, Karl Friedrich 132

Szelinski, Fritz 275f., 1213

Taneff, Wassili 957

Tegtmeyer, Werner 1077

Terboven, Josef 366,587

Thierack, Georg $71,88,91,96,100,102,112$, $114,141,173,189,215,224,227,233,236$, $239,242,246,252,273$ f., $277,279,281$ ff.,
288, 292, 312, 314, 369, 534, 598, 601 f., 674 f., $688,692,703,745,758$ ff., 766,769 , $772,940,942,954,964,969,1013,1015 \mathrm{f}$., $1033,1038,1100,1105,1127,1133,1139$, 1141,1146

Thiesing, Adolf $110,112,223,244$

Thimme, Friedrich 26

Thissen, Karl 281

Thoma, Richard 452

Thomas, Erich 283, 1211

Tigges, Eduard 225, 1211

Tigges, Hans 225

Tillessen, Heinrich 325,327

Timm, Johannes 21, 231

Tittel, Alfred 1012

Toebert, Lothar 260

Töwe, Wilhelm 1002, 1011, $1015 \mathrm{f}$., $1033 \mathrm{ff}$, 1037, 1041

Torgler, Ernst $957 \mathrm{f}$.

Trautmann, Erich 226, 1212

Troeltsch, Rudolf 237, 282, 1214

Trukenmüller (Vizepräsident des Oberlandesgerichts Stuttgart) 305

Uhl, Julius 438,458

Uiberreither, Siegfried 514, 526

Ulrich, Curt v. 964,970

Ungewitter, Artur 279, 1212

Unzner, Karl v. 21, 28

Urlacher (Regierungsrat im Reichsjustizministerium) 246

Vacano, Franz v. 942, 1015, 1028, $1033 \mathrm{f}$, 1042

Vaugoin, Karl 260

Veller, Willi 353 ff., 357 f., 362

Vogel, Erich Kurt Benno 367, 372

Vogel, Max 437

Vogels, Werner 243, 264

Vogt, Paul 1012

Volck, Herbert $222 \mathrm{f}$.

Volkmar, Erich $71,106,130,177,187,198$, $242,244,927,1014$

Vollmer, Günther 284, 768, 1095, 1213

Volmer, Max 225, 1213

Wacker, Otto 240

Wackermann, Kurt 226, 1212

Wäckerle, Hilmar 635, $637 \mathrm{f}$.

Wagemann, Gustav 141

Wagner, Adolf $74,380 \mathrm{ff}$., $388 \mathrm{ff}$., $393 \mathrm{ff}$., $406 \mathrm{ff}$., $433 \mathrm{ff}$., $550 \mathrm{f}$., $558,573,586,637 \mathrm{f}$., $640,642,688,691,698,876$

Wagner, Albrecht 1,6

Wagner, Gerhard $499 \mathrm{ff}$.

Wagner, Josef 697 
Wagner, Otto 243, 245, 259, $284 \mathrm{f}$., 1214

Wagner, Robert 33, 42

Wagner, Walter $1,6 \mathrm{f}$.

Wahl, Karl 380

Waldeck und Pyrmont, Josias Erbprinz zu 435

Weber, Alfred 239, 279, 282, 932 f., 981, 983 , 1019,1212

Weber, Friedrich $33,39,44$

Weber, Hellmuth v. $826 \mathrm{f}$.

Weber, Otto 109f., 239, 850

Weddige, Dr. med. (Ministerialrat im Reichsjustizministerium) 244

Wedell, Erich 483,698

Wein, Benedikt 79

Weinkauff, Hermann 1, 3f., 1012

Weiß, Walter 83

Weiß, Wilhelm 966

Weitzel, Fritz 328, 348, 363

Welsch, Heinrich 283, 1214

Wenninger, Ralph 963

Werner, Ferdinand 240

Werner, Georg 225, 1213

Werner, Karl 964

Werner, Paul 738

Wessel, Horst 578,675

Westermann, Hans 705

Westfeld, Albert 246

Wicherts, Dr. (Landgerichtsrat in Berlin) 223

Wichmann, Bernhard 226, 1212

Widmann, Emil 232

Wiechmann, Carl 226, 1211

Wiedemann, Fritz 72, 468, 479

Wienstein, Richard Adalbert 501, 966

Wiesemann, Falk 448

Wilde, Leopold 226, 1211

Wilke, Gustav 245, 258, 276

Wille, Kurt 259
Willers, Johannes 244, 265

Willhöfft, Walter 1012

Wimmer, Wilhelm $963 \mathrm{f}$.

Winckler, Gustav $354 \mathrm{ff}$, 359 f., 362

Windhausen, Paul 1213

Wirth, Dr. (Ministerialdirektor im preußischen Justizministerium) $222 \mathrm{f}$.

Wirth, Joseph 24

Witt, Wilhelm 1012

Witte, Max 225, 1211

Wittje, Kurt 463

Wittland, Hermann 195, 259

Wittmann (Oberlandesgerichtsrat im preußischen Justizministerium) 223

Wohler, Waiter 280, 1211

Wolf, Walter 911

Wolff, Bruno 355, 358, 650, $654 \mathrm{f}$., $657 \mathrm{f}$.

Wolff, Paul 228

Woyrsch, Udo v. 459 f., 462 ff., 468

Wrede, Karl Fürst v. 382

Wurm, Theophil 522

Wurmstich, Werner 1212

Xylander, Rudolf v. 24

Ysenburg, Ferdinand Karl Prinz zu 436

Zastrow, Hans-Hermann 273

Zehnter, Karl 438, 440, 459

Zehrfeld, Fritz 245

Zeiler, Alois 853, 1012

Zieger, Georg 963

Zimmermann, Christian 39

Zindel, Karl 1017, $1020 \mathrm{f} ., 1024 \mathrm{ff}$.

Zoeller, Otto 1012

Zoref, Ernestine 440

Zoričić, Milovan 118

Zweig, Alexander $460,464,480$

Zweig, Jeanette 460,480 


\section{Sachregister}

Der Nachweis des Stichwortes Reichsjustizminister(ium) wurde auf bestimmte Sachbezüge beschränkt.

Abhörverbot von Auslandssendern $902 \mathrm{ff}$., 952, 1080, 1131

- Einziehung von Rundfunkgeräten 902 ff.

- Zahl der Verurteilten 905

Abolition s. Niederschlagung

Absberg (Franken) 532

Abschreckung 778f., 790, 895, 899, 905, 918, 924, 950, 954, 1067, 1088, 1101, 1104, 1131,1136

Abtreibung s. auch Schwangerschaftsunterbrechung $500,667,674,837,1093,1100,1141$

Abwesenheitsverfahren s. unter Strafverfahren

Adhäsionsverfahren 984, 992, 1007, 1012, 1014, 1090

Ächtung 761, 771 f., 997

Ägypten 697

Ärzte, jüdische 666

Akademie für Deutsches Recht 2, 69, 74, 88, $105,115,121,164,468,475 \mathrm{f}, 562,652$, 654, 737, $747 \mathrm{f}$., 752, $757 \mathrm{ff} ., 793,795,869$, 927, 929 f., 937, 1002 f., 1006, 1017

- Ausschuß für Strafprozeßrecht 985, 993, 1004, 1014, 1016f., 1019, 1023, $1030 \mathrm{ff}$., 1037

- Beteiligung an der Gesetzgebung 747, 793

- Denkschrift des Ausschusses für Strafprozeßrecht vom Juli 19371016

- Denkschrift des Zentralausschusses der Strafrechtsabteilung vom Juli 1934 766, 775

- Strafrechtsausschuß 758 ff., $766 \mathrm{f} ., 769 \mathrm{f}$., 793, 985

- Unterausschuß für Strafprozeßrecht 981

Akzessorietät 775,789

Allach (bei München) 439

Allgemeines Bürgerliches Gesetzbuch (Österreichs) 929

„Altanwälte“, jüdische 72, 139, 144, 146, $148 \mathrm{f}$., 175, $180 \mathrm{f} ., 188,1116$

„Altbeamte“, jüdische 138, 144, 146, 155, $170,173,230,242,249,1116$

Altenberg (Erzgebirge) 697, $1016 \mathrm{f}$.

Altersgrenze

- Festsetzung 240, 292

- Überschreitung 281, $298 \mathrm{f}$., 317
Altherrenbund der Deutschen Studenten 251

Altötting (Oberbayern) 383

Altona (Hamburg-)

- „Blutsonntag“ von 60

- Bombenlegerprozeß 222

- Polizeipräsident 363

Amnestie s. Straffreiheit

Amtsanmaßung 330,785

Amtsanwälte $107,129,221,238,289,295$, 333, 1088

Amtsgericht(e) 119, 220, 279, 291, $293 \mathrm{ff.}$, $317,516,530,576,582,591,610,613,633$, 700, 871, 932, 941, $945 \mathrm{f} ., 953 \mathrm{f} ., 960,968$, $971,973,975,989,991,1050,1061,1063$, 1069f., 1079, 1084, 1096, 1133

- Ahrweiler (Bezirk Koblenz) 495

- Alsfeld (Oberhessen) 575

- Alzenau (Mainfranken) 394

- Andernach (Bezirk Koblenz) $494 \mathrm{f}$.

- Apolda (Thüringen) 861

- Bad Kreuznach (Bezirk Koblenz) 495, 954

- Bad Sülze (Mecklenburg) $871 \mathrm{f}$.

- Bamberg 861

- Berlin 184, 298, 665, 674, 708, 848

- Berlin-Mitte 126f.

- Berlin-Schöneberg 673

- Berlin-Spandau 665

- Berlin-Wedding 665

- Bielefeld 663

- Brandenburg (Havel) 505, 512

- Breslau 322

- Bütow (Bezirk Köslin) 665

- Coesfeld (Westfalen) 515

- Dachau 633, 636

- Dessau 848

- Düren (Bezirk Aachen) 665

- Feldkirchen (Kärnten) 523

- Frankenthal (Pfalz) 235

- Gießen 899

- Halle (Saale) 685

- Hamburg 376

- Hamburg-Harburg 674

- Heide (Holstein) 337

- Hindenburg (Oberschlesien) 665

- Homburg (Saar) 861 
- Husum (Schleswig) $340 \mathrm{f}$.

- Idar-Oberstein (Bezirk Koblenz) 494

- Jena 660

- Kappeln (Schleswig) 337, 339

- Koblenz $494 \mathrm{f}$

- Kochem (Mosel) 494

- Köln 220, 229,674

- Königsberg (Ostpreußen) $871 \mathrm{f}$.

- Landshut (Bayern) 383

- Leipzig 126, 876

- Lesum (Bremen) 674

- Litzmannstadt (Lodz, Polen) 291

- Luckenwalde (Bezirk Potsdam) 662

- Lüneburg 667

- Mainz 665

- München 13, 233, 384f., 606, 861

- Neuwied (Bezirk Koblenz) 494f., $539 \mathrm{f}$.

- Nürnberg 231, 285, 515, 665

- Offenbach (Main) 420

- Oranienburg (bei Berlin) 653, 656

- Pottenstein (Oberfranken) 385 f., 398

- Reinheim (Odenwald) 575

- Schmalkalden (Thüringen) 661

- Siegburg $539 \mathrm{f}$.

- Sinzig (Rhein) 494

- Sögel (Bezirk Osnabrück) 363 f., 464

- Stadtroda (Thüringen) 861

- Stolberg (Harz) 685

- Stuttgart 511, 515,612

- Weißenfels (Halle-Merseburg) 861

- Wetzlar (Reg.-Bezirk Wiesbaden) 872

- Wien 516

- Wilhelmshaven 674

- Wolfstein (Pfalz) 383

- Zahl der 101

Amtsmißbrauch 547, 703

Amtsrichter 934, 936, 993, 1019, 1033, 1093

- als Einzelrichter 932f., 938, $940 \mathrm{ff}$., 956, 975 ff., 989, 991, 1008, 1039, 1050 f., 1061, 1065, 1069 f., 1083, 1088, 1090, 1134, 1136

Amtstracht $290 \mathrm{f}$.

Amtsverschwiegenheit $204 \mathrm{f}$., 387

Analogie 6, 200, 453, 466, 660, $763 \mathrm{ff} ., 773$, 790, 849 ff., 880, 912, 925, 976, 983, 988, 1013,1060 f., 1093, 1129f., 1136, 1141, 1144

- Anwendung in der Rechtsprechung $861 \mathrm{f}$., 912

- Bestätigung von Analogieurteilen 895

„Der Angriff“" 104, 126 f., 223, 828, 894, 896

Anhalt 112, 114, 344

Ansbach (Mittelfranken) 532

Antiliberalismus $66,722,760 \mathrm{f.}, 765,790,924$, 926, 957, 980f., 1001, 1056, 1124, 1128, 1130 f., 1137, 1146

Antisemitismus s. auch Juden 1114
Antragsdelikte 803, 815, 907, 1090

Antwerpen 660

Anwaltskammer(n) 127 ff., $136,144 \mathrm{f} ., 149$, 180 ff., 322, $566 \mathrm{f}$., 572, 694, 696, $701 \mathrm{f}$., 1014

Anwaltsorganisationen s. auch Deutscher Anwaltverein 128, 174, 176, 188, 221, 290

Anwaltszwang s. auch Verteidiger, Zulassung und Bestellung 128, 187

Arbeitsdienst s. auch Reicharbeitsdienst 216, $300 \mathrm{ff} ., 304,309,312,833$

Arbeitserziehungslager 690

Arbeitsgericht(e) 175, $1053 \mathrm{f}$.

Arbeitshaus 675, $735 \mathrm{ff} ., 740,745,777,784 \mathrm{f}$, $841 \mathrm{f}$., 992, 1026

- Kislau 738

- St. Georgen-Bayreuth 738

- Vechta 738

Arbeitsrecht 310, 927, 929, 1132

Arbeitsverweigerung 583, 689f., 692, 694, $718,727,739,743,762,784,841,1088 \mathrm{f}$., 1125,1137

„Arisierung“ 367,695 ff., $1115 \mathrm{ff}$.

Armenanwälte $148,176,188$

- jüdische 128, 163, 180, 188

Aschaffenburg $394 \mathrm{f}$.

Asoziale 233, 509, 718 f., 725 ff., 734 ff., 743 , $745,808,841,1125$

- Bewahrungsgesetz 737

Assessoren 87, 140f., 202, 217 f., 235, 238, 253, 259, 267, 293, 296, 302 f., 312 ff., 341, $443 \mathrm{ff} ., 668,670,954,975,1114,1133$

- jüdische 128, 140, $165 \mathrm{ff}$., $170 \mathrm{f}$.

- Kriegsassessoren 318

- wirtschaftliche Lage 315 f., 1114

- Zahl der $315 \mathrm{f}$., 318

Assessorinnen 218, $268 \mathrm{f} ., 318 \mathrm{f}$.

- Zahl der 318

Augsburg 438, 635

Ausbildung s. auch Justizausbildungsordnung 5, 91, 106 ff., 238, 270, 292 f., 295 ff., 299 ff., $1115,1117,1137,1139$

Auslandstat(en) $780 \mathrm{f} ., 832,845,862,877,880$, $915 \mathrm{f}$.

- von Ausländern 780, 916, 1131

Auslieferung 1095

Ausnahmegericht(e) 950, 959

Ausnahmezustand 18f., 32, 51, 62, $65 \mathrm{f}$., 133, $137,161,322,324,327,536,545,548,676$, $678,1119,1122,1124,1127,1143$

Aussage(n)

- Bestrafung der unwahren 1004, 1056

- Erpressung der, s. auch „Verschärfte Vernehmung“" 352, 361, 694, 703 ff., 883, 1032 , 1081,1127

- Verweigerung 703, $713 \mathrm{f}$. 
Außerordentlicher Einspruch 6, 680, 684, 910f., 913, 944, 970, 1037, 1039f., 1072, $1074 \mathrm{ff} ., 1083,1085,1088,1109 \mathrm{f}$., $1135 \mathrm{f}$.

- Handhabung in der Praxis $1078 \mathrm{ff}$.

- Zahl der Fälle 1079 ff., 1137

Aussperrungsverbot 762, 782, 801

Auswärtiges Amt 697, 772, 799, 824, 834, $863,865,868,870,891,1011,1055$

Ausweisung $661,761,836,842,870$

- Hitlers 36f., 39, 41, 236

Autoritäre Ordnung 13, 17, 32, 49f., 58, $62 \mathrm{f}$., 65 ff., $545,569,752,790,847,850,853,860$, 980 f., $984,995,1001,1003,1113 \mathrm{ff}$., 1121, $1124,1144,1146$

Bad Brückenau (Unterfranken) 816, 1017, 1025, 1027, 1039

Bad Schwalbach (Bezirk Wiesbaden) 1017

Bad Wiessee (Oberbayern) 434, $458 \mathrm{f}$.

Baden 98, 110, 112, 135, 147, 213, 240, 245, $301,614,616 \mathrm{f}$.

Bamberg 16, 395, 634

Bansin (Usedom) 772

Bauernrecht 244, 310, 927, 1133

Bayerische Mittelpartei (deutsch-national) 21, 23 f., 47, 253

Bayerische Politische Polizei $235 \mathrm{f}$., $380 \mathrm{ff}$., $387,390 \mathrm{f} ., 394,405 \mathrm{ff}$., $424,437 \mathrm{ff} ., 440 \mathrm{f}$, $464,548 \mathrm{f}$, 569, 575, 585, 589, 593, 599, 610 f., 619,633 ff., $641,643,645,700,726$, 958

Bayerische Staatszeitung 67, 822, 926

Bayerische Volkspartei (BVP) 18, 20, 23, 35, $47 \mathrm{f}$., 51 ff., 58, 236, 382, 410

„Bayerische Volkspartei-Korrespondenz“ 54

Bayerischer Bauernbund 20, $52 \mathrm{f}$.

„Bayerischer Kurier“ $53 \mathrm{ff}$.

Bayerisches Oberstes Landesgericht 12, 21, $23,27,41$ ff., 46 ff., 119, 232, 234, 237, 239, 603, 961

„Bayerisches Vaterland“ 439

Bayern 5, 14, 17 f., 20f., 26, 30 ff., 36, 47, 51 f., $55,65,68,98$ f., $107,112,114,128$ f., 135 , $144 \mathrm{f}$., $150,154,158,230 \mathrm{ff}$., 238, 240, $245 \mathrm{f}$., $274,276,322 \mathrm{ff}$., $332 \mathrm{ff}$., $336,345,380 \mathrm{ff}$, 424, $434 \mathrm{ff}$., 478, 547, $549 \mathrm{ff}$., $573 \mathrm{f}$., 586 , 614, 691 f., 698, 700 f., 722, 726, 835, 961 f., $1056,1094,1120$

Bayernbund 439

Bayreuth 431, 1073

Beamte s. Justizbeamte

Beamtenrecht 97, 107, 112, 116f., 120, 124, 129, 132 ff., 137, 161, 189 ff., 202, 290, 315, 668,867

Beauftragte des Reichsministers der Justiz s. unter Reichsjustizministerium
Befähigung zum Richteramt s. Richteramt

Befehlsnotstand 351, 466, 493

Begnadigung s. auch Gnadenrecht 14, 19, $22 \mathrm{ff}$., $26 \mathrm{ff}$., $40 \mathrm{f}$., $43,61,84,93 \mathrm{f}$., 97, 324, $326,328-336,342,352,360,373 \mathrm{f} ., 422$, $468 \mathrm{ff} ., 476,517,605,611,616,618$, $626-632,688,808,826,828,1038,1046$, $1071,1094,1119,1122$

Begünstigung 352, 377, 411, 713, 785, $881 \mathrm{ff}$.

Berchtesgaden 421, 462, 805, 812, 816, 898, 910, 1071

Berichtspflicht der Justizbehörden s. „Mitteilungen in Strafsachen " und unter Staatsanwälte

Berlin 104, 109, 111, 121, 126f., 136, $147 \mathrm{ff}$., $160,321,421,434,437,442$ ff., 458, 486, $522,661,679,698,715,738,754,758,766$, $769,805,897,960$ f., 1015, 1027, 1039, 1078

Berliner Börsen-Zeitung 724

Berliner illustrierte Nachtausgabe 686

Berliner Lokal-Anzeiger 873

Berliner Tageblatt 140, 724

Bernburg (Anhalt) 504, 526

Berufsbeamtengesetz 75, 91, $136 \mathrm{ff}$., $145 \mathrm{ff}$., $153 \mathrm{ff} ., 157 \mathrm{ff} ., 169 \mathrm{ff} ., 180,189,196,198$, 218,222 ff., $230,242,248$ f., 251,254 ff., $300,662,699,867,869,1114,1116,1118$

- Auswirkung seiner Durchführung in Zahlen $165 \mathrm{ff}$.

- Entstehung $132 \mathrm{ff}$.

Berufsverbot 842, 844, 942, 1069

Berufsverbrecher $658,689,719$ ff., 726 ff., 736 , 745, 798, 838 ff., 1000

Berufsvereinigungen der Juristen s. auch Bund Nationalsozialistischer Deutscher Juristen und Deutscher Richterbund $86 \mathrm{ff}$., 221, 250, 1116

Berufung $932 \mathrm{f} ., 936,938,943,948,952,967$, $976 \mathrm{f} ., 982$, $989 \mathrm{ff}$., $997 \mathrm{f} ., 1006,1008 \mathrm{f}$., 1011 f., 1016, 1036 f., 1039, 1058 f., 1061 ff., $1070,1083,1088,1090,1108 \mathrm{f} ., 1134 \mathrm{ff}$.

Besoldung s. unter Justizbeamte

Bewährung $28 \mathrm{ff}$., $33 \mathrm{f}$., 37, 39, $41 \mathrm{ff}$., 616, 628

Beweggründe

- ehrenhafte 776,838

- "nationale“ $19 \mathrm{f}$., $36 \mathrm{ff} ., 43,47,52,61,69$, $79,122,194,289,324$ ff., 331 f., 335,358 , $362,393,430,467,1096,1114,1119,1121$, $1143 \mathrm{f}$.

- niedrige $20,332,335,361,369,371,373$, 376, $383 \mathrm{ff}$., 412, 465, 469f., 487, 490, $492 \mathrm{f}$., 495, 639, 706, 711, 786, 814 f., 833, 836, $838,879,910,1120 \mathrm{ff}$.

- politische $19,26,325,335,373,382 \mathrm{f}$., 400 , 404, 409, 461, $468 \mathrm{ff}$., 474f., 488, 493, 584, 
$662,809,815,823,832,904 f ., 1096,1099$, 1128

Beweisantizipation 1062, 1070f., 1136

Beweiserhebung(en) 714, 948, 958, $982 \mathrm{f}$., $987 \mathrm{ff}$., $1012 \mathrm{f} ., 1034,1036 \mathrm{f} ., 1040,1061 \mathrm{f}$., 1070, 1083, 1091, 1106, 1135f., 1140

Beweisschwierigkeiten 825, 854, 950, 952, 1091

Beweiswürdigkeit, gerichtliche 661

Bibelforscher $541 \mathrm{ff}$., 585, 589, $599 \mathrm{ff} ., 620 \mathrm{ff}$., $678,718,1098,1102,1126$

- Höhe der Strafen gegen 1100

Bielefeld 282, $623 \mathrm{f}$.

Blutschutzgesetz s. auch „Rassenschande“ 130, $169,622,659,761,770,862,864,868$, 876 ff., $881 \mathrm{f} ., 928,1093,1096 \mathrm{f} ., 1101 \mathrm{f}$., 1130,1144

- Ausführungsverordnungen $885 \mathrm{f}$.

Bolivien 676

Bolschewismus s. Kommunismus

Bonn 456, 894

„Bourse Egyptienne“ 482

Brandenburg (Havel) 504, 508 f., 517, 526

Brandenburg-Görden $511 \mathrm{f}$.

Braunschweig 112, 114, 240, 271, 299, 574

Bredow-Prozeß 350 ff., 360, 368 f., 372, 377, 473, 704, 706, 1120

Bremen 98, 107, 112, 114, 442, 458, 462, 593, 661,738

Breslau 124ff., 148, 190, 324, 459f., $463 \mathrm{f}$., $513,686,695,754,769,826$

Brieg (Bezirk Breslau) 459

Brüssel 718

„Bürgerlicher Tod“ 771

Bürgerliches Gesetzbuch (BGB) 118, 224, 544, 695, 752 f., 869, 871, 873, 925 ff., 1023, $1131 \mathrm{f}$.

- „Abschied vom“ $928 \mathrm{f}$.

Bürgerliches Recht 2, 7, 84, 86, 106, 242, 244, $258,265,506,522,549,665,753,771,865$, $869,873,878,925$ ff., 1069, 1131, 1143

- Reformkontinuität 928

Bund Nationalsozialistischer Deutscher Juristen (BNSDJ) s. auch Nationalsozialistischer Rechtswahrerbund (NSRB) 10, 86 ff., 114, $121,125 \mathrm{f} ., 128,134,145,149,163,176$, $206,210,234,242,251,272$ f., 282, 284, 286, 301, 305, 307 f., 565 ff., 695, 747, 754, 757, 759, 766, 768, 791f., 797, 849, $869 \mathrm{f}$,, $937 \mathrm{f}$, 958, 965, 984, $993 \mathrm{f} ., 1111,1116$

- Zahl der Mitglieder 221

Bundesgerichtshof (BGH) 240, 353, 464

Bunzlau (Niederschlesien) $463 \mathrm{f}$.

Ceylon 865

Chemnitz 492
China 826

Croischwitz (Niederschlesien) 459

Dachau (Oberbayern) s. auch unter Konzentrationslager $438 \mathrm{f}$., 442, 459

Danzig 275, 280, 287, 339, 742, 744, 862

- Obergericht 280, 287

Darmstadt 240

Denunziation(en) 785, 835f., 866, 879, 902, 904

Deutsche Allgemeine Zeitung 124

Deutsche Arbeitsfront (DAF) 251, 307, 312, 422, 490, 695, 797

Deutsche Demokratische Partei (DDP) 18 , $20,23,49,85,242,244$

„Deutsche Justiz“ 539, 541f., 551, 663, 666, 668 ff., 764, 799, 849, 1076, 1098

Deutsche Nationalsozialistische Arbeiterpartei (DNSAP) 278, 288

Deutsche Rechtsfront 88, 104

„Der Deutsche Rechtspfleger“ 870

Deutsche Staatspartei (DStP) 279, 288

Deutsche Strafrechtsgesellschaft 1048

Deutsche Volkspartei (DVP) 223, 240, $244 \mathrm{f}$., 266, 282 f., 288, 753

Deutscher Anwaltverein 75, 221, 323, 566

Deutscher Beamtenbund 121, 217

Deutscher Caritasverband 498

Deutscher Juristentag s. Juristentag, Deutscher

Deutscher Luftsportverband 832 f., 963

Deutscher Reichsbund für Leibesübungen 251

Deutscher Richterbund 131f., 221, 1108

Deutsches Beamtengesetz (DBG) v. 26.1.1937 120, 165, 172, 189, 191 ff., 203 ff., 207, 209, 277, 290, 752, 815f., 974, $1118 f$., 1144

- Dienststrafverfahren nach $\$ 22$ 200, 806

- Garantie des $§ 171$ 191f., $198 \mathrm{ff} ., 725$, $1118 \mathrm{f}$.

- Verfahren nach $§ 71$ 191, 193 ff., $202 \mathrm{f}$., $1118 \mathrm{f}$.

Deutsches Nachrichten-Büro (DNB) $876 \mathrm{f}$., $895 \mathrm{f}$.

Deutsches Rotes Kreuz 251, 787

Deutschnationale Volkspartei (DNVP) 9, 48, $50 \mathrm{ff} ., 56,58,63 \mathrm{f}$., 226, 243, 253, 272, 275 , 280, 285, 288, 753, 759, 1113

Deutschvölkische Arbeitsgemeinschaft 24

Deutschvölkische Freiheitspartei (DVFP) 288

Deutschvölkischer Schutz- und Trutzbund 288

Devisenstrafsachen 185f., 949, 954, 1052, 1070

Dibelius-Prozeß 77

Dienstaufsicht 40, 670, 935, 973, 1034, $1107 \mathrm{ff}$. 
Dienstaufsichtsbeschwerde 542, 544, 557, 564

Dienststrafgerichtsbarkeit 7, 196, 199f., 250, $359,364,668,702,1014,1111$

Dienststrafrecht s. auch unter Deutsches Beamtengesetz $97,116,119,164,193 \mathrm{f} ., 196$, 198 ff., 259,708 f., $888,893,1035,1048$, 1119,1122

Doppelstaat (i.S. Ernst Fraenkels) $1124 \mathrm{f}$.

Dresden 96, 102, 109, 111, 114, 442, 698, 759

Düsseldorf 111, 328, $356 \mathrm{ff} .$, 704, 715, 754, 965

Duisburg 521, 704, 712

Eberswalde (Heilanstalt) 512

Ehebruch 782, 797, 803

Eherecht s. auch „Mischehe(n)“ 865 ff., 871 ff., 877 ff., 928 f., 1102, 1130

Eher-Verlag $\mathbf{4 4 0}$

Ehrenrechte $771 \mathrm{f}$., 776, 787, 799f., 836, 862, 864, 965, 1052, 1099, 1105

Ehrenschutz 809f., 814 ff., 1009

Eichberg (Landesheilanstalt) 519, 525

Eid

- Ablehnung durch die Bibelforscher 621

- Abnahme durch die ermittelnde Polizei 597

- der Hilfspolizeibeamten 388

- der (Justiz-)Beamten 20 f., 170, 173, 206, 249,1055

- der Laienrichter 372

- der SS 466

- im Gerichtsverfahren 522, 852, $883 \mathrm{f}$., 986 f., 989, 1013 f., 1040, 1053 ff., 1135

- Nacheid 1053, 1055, 1135

- religiöser $1054 \mathrm{f}$.

Eiserne Front 249

Eisernes Kreuz 15, 194, 262, 461, 482

Elberfeld (Wuppertal-) 704

Emigranten s. „Schulungshaft“

Emminger-Novelle von 1924 31, 35, 933

England 922

„Entartung“ 778, 844, 872

Entmannung 657, 736, 777, 842 ff., 936, 942, 977, 979, 992, 1058, 1069, 1093, 1134

- Zahlen 844

Erbgesundheitsgerichtsbarkeit 1009, 1123

Erbgesundheitsrecht $310,313,498 \mathrm{ff}$., $657 \mathrm{f}$., 753

Erbhofrecht 113, 268, 313

Erbrecht, Gesetzgebung 928

Erfurt 282, 286

Erlaß (in zeitlicher Reihenfolge:)

- Görings zur Förderung der nationalen Bewegung v. 17.2.1933 s. „Schießerlaß“ Görings

- Grauerts v. 29.5.1933 s. „Grauertscher Erlaß"
- des Führers und Reichskanzlers über die Ausübung des Gnadenrechts v. 1.2.1935 $335,617,627$

- des Führers und Reichskanzlers über die Ernennung und Entlassung der Reichsbeamten vom 1.2.1935 119, 208, 968

- des Führers und Reichskanzlers über die Amtstracht in der Justizverwaltung v. 19.6.1936 290

- des Führers und Reichskanzlers über die Ernennung der Beamten und die Beendigung des Beamtenverhältnisses $v$. 10.7. 1937209

- des Führers und Reichskanzlers über die Vereidigung der Beamten des Landes Österreich v. 15.3.1938 173

- des Führers und Reichskanzlers über die Einführung einer Beamtenuniform v. 3.3. 1939291

- des Führers und Reichskanzlers über die Gewährung von Straffreiheit v. 7.6.1939 s. unter Straffreiheit

- des Führers über die Bildung eines Ministerrats für die Reichsverteidigung $v$. 30.8.1939 820

- des Führers über die Vereinfachung der Rechtspflege v. 21.3.1942 1090

- des Führers über besondere Vollmachten des Reichsministers der Justiz v. 20.8.1942 1146

- des Führers über die Reichsgesetzgebung v. 10.5.1943 749

Ermächtigungsgesetz 50,67 f., 130, 133, 749, 792, 826, 829f., 834, 862, 1113, 1128, 1144

Ermessen, richterliches $839,841 \mathrm{f} ., 847,851$, 892, 908, 911, 977, 983, 988f., 1014, 1036, 1050, 1053, 1061, 1064, 1066, 1070 f., 1074, $1084,1089 \mathrm{f} ., 1135 \mathrm{ff}$.

Ermittlungsrichter s. unter Richter

Eröffnungsbeschluß $678,940,948,960,978$, $981 \mathrm{f.}, 987 \mathrm{f}$., $1005 \mathrm{f}$., $1012 \mathrm{f}$., 1016, $1033 \mathrm{f}$., 1050 ff., 1076, 1090, 1132, 1136

Erpresserischer Kindesraub 776, 782, $894 \mathrm{ff}$., 898, 900, 917, 952, 1110, 1140

Erpresserischer Menschenraub 789

Erpressung 788, 866, 879, 896, 898, 917, $1064 \mathrm{f}$.

„Erschießung auf der Flucht“ 363, 366, 634 ff., $646 \mathrm{f} ., 649 \mathrm{ff} ., 653,655$ f., $675,684,899$, 1126

Essen 282

Etat s. Haushalt

Euthanasie 5, 81, 497-534, 685, 762, 786, 1093, 1123

- „Erlaß" Hitlers v. 1.9.1939 502, 509, $512 \mathrm{f}$., $528,530,532,534,1123$ 
- Frage der gesetzlichen Regelung $507 \mathrm{ff} ., 528,530,533 \mathrm{f} ., 1123$

- wilde 524, $533 \mathrm{f}$.

- Zahl der Opfer 504, 534

Exekution(en) s. auch Polizei, Exekutionen 82, 435 ff., 455, 457, 459, 514, 533

Exemtion 412, 414, 428, $433 \mathrm{ff} ., 475,500$, $534,654,808,813,816,1046 \mathrm{ff} ., 1121$

Fachschaften s. unter Reichsbund der Deutschen Beamten (RDB)

Familienrecht, Reform $928 \mathrm{f}$.

Faschismustheorie $3 \mathrm{f}$.

Fechenbach-Prozeß 25-29, 38

Feldberg (Schwarzwald) 431

Feldmoching (bei München) 384

Feststellungshaft (zur Identifizierung der Person) $736,989,1029$

Feststellungsverfahren (bei ehrenrührigen Behauptungen) 984, 992, 1006

Festungshaft 28, 33, 36, 42, 44, $47 \mathrm{f} ., 77,328$, 776, 799, $837 \mathrm{f}$., $845 \mathrm{ff}$., 977, 1047

Finanzministerium, preußisches s. unter PreuBen

Fischbachau (Oberbayern) 767 f., 773

Fischhausen (Oberbayern) 434

Fluchtgefahr(-verdacht) $326,561,586,708$, 989, 1057, 1065

Fortgesetzte Handlung 728, 737, $779 \mathrm{f}$.

„Fränkischer Kurier“ 69, 83

Frankfurt (Main) 148, 162

Frankfurt (Oder) 675

Frankfurter Zeitung 228, 725, 1048

Frauen

- Ausschluß als Laienrichter 983

- im Justizdienst s. auch Justizbeamte, weibliche 218, 268f., $318 \mathrm{f}$.

Freiheitsberaubung 330, 337, 341, 425, 467, $547,549,575,787,813,1081$

Freikorps 18, 383

Freimaurer 181, $208 \mathrm{f} ., 250$

Freising (Oberbayern) 513

Freiwillige Gerichtsbarkeit 84, 113, 319, 515, 522

„Fremdvölkische“ 534, 868, 1131

„Friedensbuße“ 993

Friedensrichter 88, 993

Friedensrichter- und Schiedsmannsordnung 983, 985, 992 ff., 1007, 1012, 1017, 1090

Fritsch-Prozeß 76, 256

Fron 777

Frontkämpfer, jüdische $72,127,132,134$, 138 ff., 142 ff., 148 ff., 153,158 f., 169 f., 173 , 175 ff., 180 f., $188,230,249,254$ ff., 461 , 482,1116

Fuchs-Machhaus-Prozeß 37
Führererlaß s. auch unter Erlaß 751, 820

"Führergewalt“, souveräne 5, 192, $194 \mathrm{f}$., $749 \mathrm{ff} ., 1076,1102,1123 \mathrm{f}$.

Führerprinzip $628,749,873,934,937 \mathrm{f}$, $983 \mathrm{f} ., 987,996 \mathrm{ff} ., 1001 \mathrm{ff} ., 1008,1013 \mathrm{f}$., $1034 \mathrm{ff} ., 1062,1134 \mathrm{f}$

Fuhlsbüttel (Hamburg-) 374 ff., 574

Gauleiter 96, 121, 153, 155 f., 182, 207, 231, 302, 311, 380f., 397, 474, 485, 489, 496, 504, 549, 784, 946, 980, 1093, $1115 \mathrm{f} ., 1118$

- Baden 213

- Bayerische Ostmark 276, 380

- Berlin 288

- Düsseldorf 356 f., 965

- Essen 366, 587

- Franken (bis 1928: Mittelfranken) 231, 274, $285,367,380,394,404,870$

- Halle-Merseburg 213, 283, 685

- Hamburg 274, 374, 488, 669, 705

- Hessen-Nassau 214, 245, 259, 271 f., 279

- Kurmark 228, 662

- Mainfranken 276

- Mecklenburg $272 \mathrm{f}$.

- München-Oberbayern 276f., 380, 388, 433, 486, 688, 695, 876

- Ostmark (Kurmark) 222, 226

- Ostpreußen 228, 275, 283, 287

- Pommern 226f., 350

- Posen 280

- Rheinpfalz 323, 380, $383 \mathrm{f}$.

- Saarpfalz 214, 263

- Sachsen 279, 282, 304, 368 ff., 1111

- Schlesien 193, 274, 463, 465, 696f.

- Schleswig-Holstein 156, 228, 286, 338, 340

- Schwaben 380

- Steiermark 514, 526

- Tirol-Vorarlberg 278, 286

- Thüringen 109, 207

- Unterfranken (ab 1935: Mainfranken) 380

- Weser-Ems 279, 362, 364f.

- Westfalen-Nord 282

- Westfalen-Süd 282

- Württemberg-Hohenzollern 305

- Eingriffe in die Justiz 121, 228, 350, $364 \mathrm{f}$.

- Mitwirkung bei der Personalpolitik 121, 207 ff., 212 ff., 226, 230 f., 271-288

Gefängnisgeistliche 297, 576

Geheime Staatspolizei s. Gestapo

Geheimes Staatspolizeiamt 287, 330, $345 \mathrm{ff}$., $350,380,410,434,438,440,442,444,456$, $460,478 \mathrm{ff} ., 537,540,542,544,550,554 \mathrm{f}$., 560 ff., 565 ff., $570,572,576,578,582$, 585 f., $588,594,600$ ff., 608 f., $612,616,618$, $623,627 \mathrm{f}$., 633,645 f., 653,659 f., $675 \mathrm{ff}$., $683,695,699$ ff., $708,710,713,716$ ff., 720 , 
871, 874f., 879, 884, 886, 888, 993, 1020

- Badisches 576,615,617

- Darmstadt 575f.

- Lübeck $577 \mathrm{ff}$.

- Sachsen 369f., 372, 620

Geheimhaltungspflicht $809,814 \mathrm{ff}$.

Geheimnisverrat $886,888 \mathrm{ff}$., 893

Gehorsamspflicht $204 \mathrm{ff}$.

Geiselnahme 18, 823, 896, 947

Geistliche s. Gefängnisgeistliche und Kirche

Geldstrafe(n) 328, 415, 420, 542, 621, 662, $777,789,797,823,833,843,845,918$, 1047, 1052, $1099 \mathrm{f}$.

Gemeinnützige Kranken-Transport-GmbH $504,515,517,525$

Gemeinnützige Stiftung für Anstaltspflege 504

Gemeinschaftslager „Hanns Kerrl“ 111, 216, 261, 301, 303-312

Generalbevollmächtigter

- für die Reichsverwaltung 268, 820, 903, 912, 915, 922, 953, 976 ff., 1082 f., 1089

- für die Wirtschaft $820,912,914$

Generalgouvernement 81f., 265, 915

Generalgouverneur (Polen) 234f., 820

Generalklausel(n) 731 f., 753, 812, 843, 869, 889, 893, 908, 916f., 925f., 929, $1131 \mathrm{f}$., 1138

Generalprävention $47,674,790,866,894 \mathrm{f}$, 1025, 1129

Generalstaatsanwälte $97,112 \mathrm{f} ., 119,121$, 127 ff., 149, 155, 163, 166, 170, 209, 212 ff., 217, 222, 225f., 229, 236f., 239, 263, 266, 269 ff., 281,288 ff., 301, 327, 330, 334, 364, $401,472,476,485,492,514,518,523 \mathrm{ff}$., $530,578 \mathrm{f} ., 583,587,593,603,605,609$, $618,620,623 \mathrm{f}$., $627,629 \mathrm{ff} ., 688 \mathrm{f} ., 713,721$, $728 f ., 742$ ff., 791 f., $879 f$ f, 883, 885, 905 , 909, 914, 917, 924, $953 \mathrm{ff} ., 1014,1034$, 1037, 1056 f., 1079, 1088 f., 1092 ff., 1096 ff., 1118, 1123, 1137, 1141

- Berichte an das Reichsjustizministerium s. Lageberichte und Staatsanwälte, Berichtspflicht

- preußische 541

- Sonderdezernenten für Sondergerichtssachen $954 \mathrm{f}$.

- Tagung v. 3.4.1935 791

- Tagung v. 23.9.1935 576f., 579, 593, 599, 1094, $1099 \mathrm{f}$.

- Tagung v. 29.11.1935 $723 \mathrm{f}$.

- Tagung v. 13./14.11.1936 664, 728, 881, $1100 \mathrm{ff}$.

- Tagung v. 18.6.1937 213, 216f., 313, 563, $601,620,623,1100,1102$

- Tagung v. 7./8.3.1938 $727 \mathrm{ff} ., 1102,1105$
- Tagung v. 23. bis $25.1 .1939489 \mathrm{ff} ., 496$, $591 \mathrm{f}$., $624 \mathrm{ff} ., 885,1102$

- Tagung v. 11.9.1940 596

- Tagung v. 20.3.1941 692

- Tagung v. 23./24.4.1941 312f., 527 ff., 531, 598,1123

- Tagung v. 31.3.1942 216

- Tagung v. 29.9.1942 1139

- Tagung v. 10./11.2. $1943 \quad 292,692$

- Tagung der bayerischen v. 31.3.1933 231

- Tagung der bayerischen v. 5./6.4.1934 399, 407 f., 427 ff., 1099

- Tagung der bayerischen v. 7./8.12.1934 411, 477, 1099

- Tagung der preußischen v. 22.7.1933 1099

- Tagung der preußischen v. 12.7.1934 457, 462, 473

Generalstaatsanwalt 574,616, $1065 \mathrm{f} ., 1085 \mathrm{f}$.

- Bamberg 236, 386, 688, $691 \mathrm{f}$.

- Bayerisches Oberstes Landesgericht 237

- Berlin (Landgericht) $225 \mathrm{ff} ., 283,287,634$, 648 ff., 653,703 f., 707 ff., 876, 883, 1015 , 1024

- Braunschweig 240, 574, 576, 579, 599, 625

- Breslau 222, 226f., 281, 286f., 460 ff., 468 , 583, 734, 744, 792, 883, 1056, 1107

- Celle 226, 283 f., 365 f., 622, 689, $691 \mathrm{ff}$., 935, 981, 1019, 1056

- Danzig 287

- Darmstadt 240, 245, $271 \mathrm{f} ., 581,664,668 \mathrm{f}$.

- Dresden 239, 279, 282, 370, 374, 515, 517 f., 525 f., 581,589 ff., $617,620,694,745$, $981,1019,1056$

- Düsseldorf 226, 284, 328, 359f., $517 \mathrm{f}$., 530 f., 586, 694, 704, 710 ff., $717,733 \mathrm{f}$.

- Frankfurt a.M. 213, 226, 499, 504, 509f., 524, 526, 529f., 883

- Graz 285, 514, 526, 610

- Hamburg 92, 240, 367, 375 ff., 477, 488, 490, 496, 577 f., 604 f., 705, 712, 725, 727 f., $832,884,949,1056,1081,1106$

- Hamm 223f., 226, 274, 281 f., 529, 691, $694,715,792,1056$

- Innsbruck $285 \mathrm{f}$., 610

- Jena 239, 517, 525, $690 \mathrm{f}$.

- Kammergericht 226, 281, 283, 304, 344, $346,443,445 f ., 526,529,586,603,634$, $656,686,688,703$ f., 707, 709, 715, 876, $897,1101 \mathrm{f}$.

- Karlsruhe 240, 283, 287, 493, 530, 576, 579 ff., 595,614 ff., 694, 881, 883 f., 971 , 1056

- Kassel 226,694

- Kiel 226, 228, 286f., 338 ff., 413

- Köln 226, 531, 594, 688, 954, 1056 
- Königsberg 226, 228, 283f., 287, 617, $688 \mathrm{f}$., 1056

- Leitmeritz 694

- Linz 286, 515, 520, 523, 526, 610, 694, 1087

- Marienwerder 226

- München 236f., 285, 384f., 400, 407, 411f., 435 f., 438 ff., 459, 471, 479, 490, 496, 575, $578 \mathrm{f} ., 607,619,635 \mathrm{ff} ., 639,642 \mathrm{ff} ., 688$, 885, 1056

- Naumburg 213, 226, 283, 331, 517, 526, 576, 588, 595 f., 598, 611, 613, 625, 688f., 694,792

- Nürnberg 237, 274, 282, 285, 387 f., 390 f., $393,405,517,656,681$ f., 694

- Oldenburg 239

- Posen $287 \mathrm{f}$.

- Prag 287

- Rostock 240, 273

- Stettin 226, 281, 601, 620

- Stuttgart 240, 259, 284, 507 f., 510f., 522, 526, 666 f., 669, 689, 694, 1056

- Wien 286, 515, 526,610

- Zweibrücken 237, 283, $383 \mathrm{f}$.

„Der gerade Weg“ 440

Gerechtigkeit

- formelle 850,924

- materielle 774, 850, 925, 983, 1036, 1063

Gericht(e) 290, 538 ff., 543 f., 557, 618 f., 623 , $679,728,743,871$ ff., 907, 917, 1060, 1143

- Abstimmung in Kollegialgerichten 934, 937 f., 984, 987, 996 f., 1012 f., 1016, 1034 ff., 1062,1135

- Aufbau und Organisation $931 \mathrm{ff} ., 935 \mathrm{f}$., 939 f., 942 ff., 974 ff., 1044, 1069

- Instrumente des Unrechtsstaates 1143

- Präsidialverfassung 971 ff., 1133

- Rechte des Vorsitzenden 934 f., 937 f., 948, 961, 973, 979, 982 ff., $987 \mathrm{f}$., 996, 999, 1004, $1013,1034 \mathrm{f} ., 1062,1070,1078,1083$, $1089 \mathrm{f}$., $1134 \mathrm{ff}$.

- Zahl der 119, 237

Gerichtsverfassung 6, 94, 119, 198, 202, 299, $317,819,974,1049,1069,1107$ f., 1112 , 1132 ff., 1138

- Gesetzgebung 944-980

- Reform 931-944, 974, $1132 \mathrm{ff}$.

Gerichtsverfassungsgesetz (GVG) 37, 119, 196, 206, 240, 281, 292, 346, 405, 409, 415, 424, 462, 843, 931 ff., 935, $938 \mathrm{f} ., 942,945$, 951 f., $956,967 \mathrm{f} ., 971 \mathrm{ff} ., 975,977,981,984$, 997, 1063, 1109 ff., 1132 f., 1142

- Entwurf v. 11.3.1935 $933 \mathrm{ff}$.

- Entwurf v. 30.4.1935 935

- Entwurf v. 27.2.1936 935ff., 967, 994, 996, 1012
Gerichtsverfassungsrecht 741, 931, 966 f., 980 , 1091, 1133

- Zuständigkeitsregelung durch die Verordnung v. 21.2.1940 $977 \mathrm{ff}$.

Gerichtsvollzieher 88, 107, 296

„Germania“ 538

Geschäftsverteilung

- bei den Gerichten $37 \mathrm{f} ., 130,162,196,202$, 934 f., 947, 953, 971 ff., 1005, 1119, 1133

- bei den Staatsanwaltschaften 113

- im Reichsjustizministerium 106, 244, 258, 347, 1095, 1149-1210

Geschworene 832, $933 \mathrm{ff}$., $938 \mathrm{f}$., $945 \mathrm{f} ., 949$, $975 \mathrm{f.}, 983,1093,1133$

- jüdische 128

Gesetz (in zeitlicher Reihenfolge:)

- Reichsbeamtengesetz v. 31.3.1873 s. dort

- Preßgesetz v. 5.5.1874 27

- Reichsgesetz über die Beurkundung des Personenstandes und die Eheschließung v. 6.2.1875 (Personenstandsgesetz) 871, 878

- gegen den verbrecherischen und gemeingefährlichen Gebrauch von Sprengstoffen v. 9.6.1884 326

- gegen Verrat militärischer Geheimnisse v. 8.6.1893/3.6.1914 844

- über die Bestrafung der Entziehung elektrischer Arbeit v. 9.4.1900 788, 848

- betreffend die Verhaftung und Aufenthaltsbeschränkung auf Grund des Kriegszustandes und des Belagerungszustandes v. 4.12. 1916545

- zur Ergänzung des Gesetzes zur Verfolgung von Kriegsverbrechen und Kriegsvergehen vom 18. Dezember 1919, v. 24.3.1920 $834 \mathrm{f}$.

- Wehrgesetz v. 23.3.1921 963

- über die Zulassung der Frauen zu den Ämtern und Berufen der Rechtspflege $v$. 11.7.1922 317

- Jugendgerichtsgesetz v. 16.2.1923 774

- Arbeitsgerichtsgesetz v. 23.12.1926 1053

- zur Abänderung der Strafprozeßordnung v. 27.12.1926 1059

- Besoldungsgesetz v. 16.12.1927 292

- zum Schutz der deutschen Nation, Entwurf v. 13.3 .1930864

- preußisches, Polizeiverwaltungsgesetz v. 1.6.1931 s. dort

- über Straffreiheit v. 20.12.1932 s. unter Straffreiheit

- zur Behebung der Not von Volk und Reich v. 24.3.1933 s. Ermächtigungsgesetz

- über Verhängung und Vollzug der Todesstrafe v. $29.3 .193368,764,776,826 \mathrm{ff}$., $834,836,851,894,957,959,1128$ 
- zur Gleichschaltung der Länder mit dem Reich v. 31.3.1933 834

- zur Abwehr politischer Gewalttaten v. 4.4.1933 327, 831f., 900, 949

- über die Neuwahl der Schöffen, Geschworenen und Handelsrichter v. 7.4.1933 945, 1133

- über die Zulassung zur Rechtsanwaltschaft vom 7.4.193370,91, 136f., 139ff., $144 \mathrm{ff}$, $148 \mathrm{ff} ., 157 \mathrm{ff} ., 162 \mathrm{f} ., 174,180,188,867$, 1116,1118

- zur Wiederherstellung des Berufsbeamtentums vom 7.4.1933 s. Berufsbeamtengesetz

- über das Schlachten von Tieren v. 21.4. 1933783

- betreffend die Zulassung zur Patentanwaltschaft und zur Rechtsanwaltschaft v. 22.4. 1933139,162

- bayerisches, zur Bekämpfung der Korruption v. 26.4. 1933834,838

- bayerisches, zur Bekämpfung des Angebertums v. 26.4.1933 834, 838

- betreffend die Dienststrafgewalt über die Mitglieder der SA und SS v. 28.4.1933 414, 416, $418 \mathrm{f}$., 424

- über Wiedereinführung der Militärgerichtsbarkeit v. 12.5.1933 414

- zur Abänderung strafrechtlicher Vorschriften v. 26.5. $1933833 \mathrm{ff} ., 842,844 \mathrm{ff}$.

- gegen Verrat der Deutschen Volkswirtschaft v. 12.6.1933 949, 1052f., 1066

- zur Änderung des Gesetzes zur Wiederherstellung des Berufsbeamtentums v. 23.6. 1933 160, 162

- Danziger Ermächtigungsgesetz v. 24.6. 1933862

- zur Änderung von Vorschriften auf dem Gebiete des allgemeinen Beamten-, Besoldungs- und des Versorgungsrechts vom 30.6.1933 112, 189, 300

- zur Änderung der Vorschriften des Gerichtsverfassungsgesetzes über die Präsidien der Gerichte v. 4.7.1933 972

- gegen die Neubildung von Parteien v. 14.7.1933965

- zur Verhütung erbkranken Nachwuchses v. 14.7.1933 498, 500, 870

- Zwangskartellgesetz v. 15.7.1933927

- zur Ergänzung des Gesetzes zur Wiederherstellung des Berufsbeamtentums v. 20.7. 1933159

- Reichsnährstandgesetz v. 13.9.1933927

- zur Änderung des Gesetzes zur Wiederherstellung des Berufsbeamtentums v. 22.9. 1933162

- Reichserbhofgesetz v. 29.9.1933927f.
- zur Gewährleistung des Rechtsfriedens v. 13.10.1933 92, 327, 331, 680, 831 f., 844, 897 f., 900,911 ff., 950

- zur Änderung des Verfahrens in bürgerlichen Rechtsstreitigkeiten v. 27.10.1933 1053

- gegen gefährliche Gewohnheitsverbrecher und über Maßregeln der Sicherung und Besserung v. 24.11.1933 516, $719 \mathrm{f}$., 723 , $729,731,733,736,744,774,777,779,784$, 838-844, 851, 909, 956, 1025, 1057 ff., 1063, 1065, 1096, 1128

- zur Einschränkung der Eide im Strafverfahren v. $24.11 .19331053 \mathrm{ff}$.

- preußisches, über die Geheime Staatspolizei v. $30.11 .1933456,552$ f., 699

- zur Sicherung der Einheit von Partei und Staat v. 1.12.1933 345, 402, 424 ff., 429, 748

- über die zweite Änderung der Reichshaushaltsordnung und die zehnte Änderung des Besoldungsgesetzes v. 13.12.1933 984

- sächsisches, zur Änderung des Gesetzes über die Verwaltungsrechtspflege v. 14.12. 1933537

- zur Ordnung der nationalen Arbeit v. 20.1.1934 927

- über den Neuaufbau des Reichs vom 30.1.1934 92 f., 99

- zur Überleitung der Rechtspflege auf das Reich vom 16.2.1934 93f., 96, 644

- zur Änderung des Gesetzes zur Wiederherstellung des Berufsbeamtentums v. 22.3. 1934164

- über Reichsverweisungen v. 23.3.1934 777

- zur Änderung von Vorschriften des Strafrechts und Strafverfahrens v. 24.4.1934 571, 614, 776, 824, $844 \mathrm{ff} ., 900,959 \mathrm{ff}$, 966 f., 988, 1059 f., 1132

- Änderungsgesetz zum Gesetz zur Sicherung der Einheit von Partei und Staat v. 3.7.1934 478, 748

- Reichsjagdgesetz v. 3.7.1934 854

- über Maßnahmen der Staatsnotwehr v. 3.7.1934 438, 441, $448 \mathrm{ff} ., 455 \mathrm{f}$., $458 \mathrm{f}$., 464, 471, 1122

- zur Úberleitung des Forst- und Jagdwesens auf das Reich v. 3.7.1934 462

- über die Gewährung von Straffreiheit v. 7.8.1934 s. unter Straffreiheit

- über die Vereidigung der Beamten und Soldaten der Wehrmacht v. 20.8.1934 206

- zur Anderung des Militärstrafgesetzbuchs und der Militärstrafgerichtsordnung $v$. 23.11.1934 960

- zur Überleitung der Rechtspflege auf das Reich vom 5.12.1934 111 f., 116 
- über den Ausgleich bürgerlich-rechtlicher Ansprüche v. 13.12.1934 $482 \mathrm{f}$.

- zur Änderung des Gerichtsverfassungsgesetzes v. 13.12.1934 934, 945 f., 1133

- gegen heimtückische Angriffe auf Staat und Partei und zum Schutz der Parteiuniformen v. 20.12.1934 s. auch Heimtückeverfahren 495 f., 520, 633, 661 f., 685, 743, 784, 825, $832 \mathrm{f}$., 854, 886, 889, 900, 919, 921, $950 \mathrm{f}$., 999, 1092, 1096, 1106, 1111

- zur Úberleitung der Rechtspflege auf das Reich vom 24.1.1935 116, 190

- über die Devisenbewirtschaftung v. 4.2. 19351070

- Wehrgesetz v. 21.5.1935 $873 \mathrm{f}$.

- Luftschutzgesetz v. 26.6.1935 s. Luftschutzgesetz

- zur Änderung des Gesetzes zur Verhütung erbkranken Nachwuchses v. 26.6.1935 501

- zur Änderung des Strafgesetzbuchs v. 28.6.1935 660, 763, 773, 788, $851 \mathrm{ff}$., 862 ff., $873,886,895,900,988,1060$

- zur Anderung von Vorschriften des Strafverfahrens und des Gerichtsverfassungsgesetzes v. 28.6.1935 855, 861, 863, 933, 936, $950,972,985,989,1014,1049,1052 \mathrm{f}$., 1060 ff., 1070 f., 1136

- Reichsbürgergesetz v. $15.9 .1935168 \mathrm{ff}$., 876 ff., 1117

- Reichsflaggengesetz v. 15.9.1935 $875 \mathrm{f}$.

- zum Schutze des deutschen Blutes und der deutschen Ehre v. 15.9.1935 s. Blutschutzgesetz

- zum Schutze der Erbgesundheit des deutschen Volkes (Ehegesundheitsgesetz) v. 18.10.1935 498, 928

- zur Anderung der Rechtsanwaltsordnung vom 13.12.1935 125, 174

- zur Verhütung von Mißbräuchen auf dem Gebiete der Rechtsberatung v. 13.12.1935 180

- preußisches, über die Geheime Staatspolizei v. 10.2.1936 537, 553 ff., 559, 568, 584, 598, 617 f., 1020, 1125

- über den Volksgerichtshof und über die fünfundzwanzigste Änderung des Besoldungsgesetzes v. 18.4.1936 939, 968

- über die Gewährung von Straffreiheit v. 23.4.1936 s. unter Straffreiheit

- Patentgesetz v. 5.5.1936 927,929

- gegen erpresserischen Kindesraub v. 22.6. 1936 798, 894 ff., 898 ff., 1128

- zur Anderung des Strafgesetzbuchs v. 2.7.1936 855, 886 ff., 892 ff., 900,969

- über die Statistik der Fischereifangergebnisse v. 6.7.1936 831
- über die Vorführung ausländischer Filme v. 11.7.1936 831

- gegen Wirtschaftssabotage v. 1.12 .1936 894, 900, 969

- über die Vernehmung von Angehörigen der Nationalsozialistischen Deutschen Arbeiterpartei und ihrer Gliederungen v. 1.12.1936 1068

- Aktiengesetz v. 30.1.1937 927

- zur Verlängerung des Gesetzes zur Behebung der Not von Volk und Reich v. 30.1.1937 749

- über die Geschäftsverteilung bei den Gerichten v. 24.11.1937 196, 202, 973 f., 1119

- über die Einunddreißigste Änderung des Besoldungsgesetzes v. 9.12.1937 293, 971

- Waffengesetz v. 28.3.1938 913, 965

- über die Gewährung von Straffreiheit v. 30.4.1938 s. unter Straffreiheit

- gegen Straßenraub mittels Autofallen v. 22.6. $1938750,806,894,897 \mathrm{ff}$., 952, 1128

- zur Vereinheitlichung des Rechts der Eheschließung und der Ehescheidung im Lande Österreich und im übrigen Reichsgebiet (Ehegesetz) v. 6.7.1938 928, 1102

- über Leistungen für Wehrzwecke v. 13.7.1938 831

- über die Errichtung von Testamenten und Erbverträgen v. 31.7.1938 748, 928

- zur Verlângerung des Gesetzes zur Behebung der Not von Volk und Reich v. 30.1.1939 749

- über den Fischereischein v. 19.4.1939 831

- über Mietverhältnisse mit Juden v. 30.4. 1939668

- über die Verschollenheit, die Todeserklärung und die Feststellung der Todeszeit v. 4.7.1939 928

- zur Änderung von Vorschriften des allgemeinen Strafverfahrens, des Wehrmachtstrafverfahrens und des Strafgesetzbuchs v. 16.9.1939 910, 918, 1071 ff., 1136

- zur Änderung des Reichsstrafgesetzbuchs v. 4.9.1941 728, 924

- zur Ergänzung der Vorschriften gegen Landesverrat v. 22.11.1942 800

Gesetze

- diskriminierende $878 \mathrm{f}$.

- mit Unrechtsgehalt 997

- über Straffreiheit s. dort

- "ungenügende“ 621, 662, 691 f., 705, 737, $740,798,834,840$ ff., 848 f., 855 f., 897 ff., $911 \mathrm{f}$., $921 \mathrm{ff} ., 928,1129 \mathrm{f}$.

Gesetzesauslegung $539 \mathrm{ff} ., \quad 584,722,731 \mathrm{f}$., 774, 798, 849 ff., 856 f., 879 ff., 898 f., 908, 
913, 923, 926f., 998, 1063, 1079f., 1095 ff., $1100 \mathrm{f} ., 1108,1132,1138 \mathrm{f} ., 1143$

Gesetzesgebundenheit $848 \mathrm{ff} ., 857,860,872 \mathrm{f}$., $914,928,935,997,1003,1130,1138,1143$

Gesetzgebung $s$. auch Reichsjustizministerium, Gesetzgebungstätigkeit $78,84 \mathrm{f}$, 90 ff., 94 ff., $100 \mathrm{f} ., 115,120,192,201,550$, 554, 746-753, 759 f., 819 f., 822 ff., 847, 853, $869,873,882,895,898,901 \mathrm{f} ., 920,927 \mathrm{f}$, 993, 1092, 1110, 1112, 1115, 1128f., 1138

- kasuistische 1138

- „kleine“ 1095

Gesetzmäßigkeit der Verwaltung $68 \mathrm{f} ., 122$, $482 \mathrm{f}$., $536 \mathrm{ff} ., 544,560,562 \mathrm{f}$., 596, 665, 693, 705, 876f., 1124, 1144

Gestapo s. auch Bayerische Politische Polizei und Staatspolizei(leit)stelle(n) $76 \mathrm{ff} ., \quad 168$, 182 ff., 197, 236, 254, 302, 324, 345, 349, $354 \mathrm{f} ., 363$ ff., 372, 374 f., 379, 442, 444, 446, $456,471,475,478 \mathrm{ff} ., 485 \mathrm{ff} ., 496,507,514$, 533,536 ff., 546 f., 550, 564 ff., 573 ff., 584 ff., $599 \mathrm{ff} ., 603 \mathrm{ff} ., 611 \mathrm{f}$., $627 \mathrm{ff} ., 694 \mathrm{ff} ., 703 \mathrm{ff}$., $871,875 f$., 879, 881, 889, 920, 923, 1029f., 1044, 1100, 1110, $1121 \mathrm{f} ., 1124$ ff., 1144

- Spitzel und V-Leute $713 \mathrm{ff} ., 1029 \mathrm{f}$.

„Gesundes Volksempfinden“ 476, 531, 673 $681,763,775,780,787,802,804,849 \mathrm{ff}$., 856,858 ff., 863, 880, 908 f., 940 f., 957,985 , 990 f., $1007,1038,1060,1066,1087,1098$, $1129 \mathrm{f} ., 1135,1138$

Gewaltenteilung 537f., 1076, 1124, 1140, 1144

- im Strafprozeß 986, 988, 1065

Gewaltverbrecher s. Verordnung gegen Gewaltverbrecher v. 5.12.1939

Gewerkschaften $12 \mathrm{f}$, $62 \mathrm{f}$., 68, 322

Gewohnheitsverbrecher s. auch Gesetz gegen gefährliche Gewohnheitsverbrecher und über Maßregeln der Sicherung und Besserung v. 24.11.1933 550, 719, $723 \mathrm{f}$., $726 \mathrm{ff}$., 732, 736 f., 743, 777 ff., 798,838 ff., 846, 909, 917, 924, 956

Gleichheit vor dem Gesetz 20, 475, 490, 605, $878,1130,1144$

Gleiwitz (Oberschlesien) 125, 734

Glogau (Niederschlesien) 460f., 463, 480

Gnadenrecht s. auch Begnadigung 7, $93 \mathrm{f}$., 113, 330, $332 \mathrm{ff}$., 347, 369, 373, $491 \mathrm{f}$., 500, $616 \mathrm{f} ., 627 \mathrm{ff} ., 715,997,1007,1046,1079$

Görlitz 125, 460

Göttingen (Heilanstalt) 512

Gogolin (bei Oppeln) 1074

Gotha 690

G.P.U. 514

Grafeneck (Tötungsanstalt) 504, 510, 526

„Grauertscher Erlaß“ 359 ff., 703
Graz 278, 285, 288, 805, 951, 1078

Großdeutsche Volkspartei (Österreich) 285, 288

Groß-Solschen (Niedersachsen) 564

Grundbuchamt 972, 974, 1133

Grundrechte, Einschränkung und Verletzung $66,122,130,536,543,545 \mathrm{f} ., 549,790,856$, 862 f., 1018, 1026, 1125, 1144

"Grundsätze der inneren Staatssicherung während des Krieges“ v. 3.9.1939 676, 689

Gunzenhausen (Mittelfranken) $403 \mathrm{ff}$.

\section{Hadamar (Tötungsanstalt) 504, 526}

Haftbefehl 78, 331, 337, 340, 383, 387, 394, $397,401,420,545,548 \mathrm{f} ., 557,560,564$, $576,580,584$ ff., 590, 599, 635f., 638, 661, 708f., 948, $986 \mathrm{f} ., 1001,1005,1012,1014$, 1016, 1019f., 1023f., 1033, 1057, 1059f., $1066,1083,1090,1135$

Haftprüfung $589,726,728,1050,1059 f$, 1136

Hahnenklee (Harz) 556f., 769 f., 772

Halbjuden 169, 173, 177, $180 \mathrm{f} ., 189,242$, $877 \mathrm{f}$.

Hamburg 98, 107, 109 ff., 114, 158, 238, 245, $253,301,314,374 \mathrm{ff} ., 477,594,604,612$, $626,667,672,705,831,1081$

- Besprechung der Justizbeamten v. 28.1 . 1939672

- Besprechung der Gerichtspräsidenten v. 1.2.1939 672

Handelsrecht 927, 1132

Handelsrichter, jüdische 129

Hannover 104, 206, 377, 484, 758

„Hans-Frank-Lager“ 301

Harstedt (Bremen-) 593

Hartheim (Tötungsanstalt) 504f., 519, 524, 526

Harzburger Front 52

Hauptverfahren 585, 732, 1041, 1051,1096

- Eröffnung s. Eröffnungsbeschluß

- Vorsitzender als „Herr des Hauptverfahrens“ 987, 1014, 1037

Hauptverhandlung 712f., 885, 897, $933 \mathrm{f}$., 936f., 942, $948 \mathrm{f} ., 952,955,958,961,970$, 976, $978 \mathrm{ff} ., 987 \mathrm{f} ., 1000,1004,1006,1029$, $1031,1033 \mathrm{f} ., 1039,1050 \mathrm{ff} ., 1058,1060 \mathrm{f}$, $1066 \mathrm{f} ., 1069,1072 \mathrm{f} ., 1075 \mathrm{f},, 1078,1083 \mathrm{f}$., $1093 \mathrm{f}$., $1105 \mathrm{f}$., $1108 \mathrm{ff}$., 1127, $1135 \mathrm{f} ., 1140$, 1142

- Ausschließung des Angeklagten $1029 \mathrm{f}$.

- Mündlichkeit und Unmittelbarkeit 982, 986, 990, 1140

- Verweis einer Strafsache an den Besonderen Senat des Reichsgerichts in der Hauptverhandlung $1041 \mathrm{ff}$. 
Haushalt $86,97,105 \mathrm{ff} ., 112,116 \mathrm{ff} ., 161,238$, 244, 264f., 292, 553, 559, 607, 720, 938, $966 \mathrm{f}$.

Heide (Holstein) $337 \mathrm{f}$.

Heidelberg 928

Heimtückeverfahren s. auch Verordnung zur Abwehr heimtückischer Angriffe gegen die Regierung der nationalen Erhebung v. 21.3.1933 und Gesetz gegen heimtückische Angriffe auf Staat und Partei und zum Schutz der Parteiuniformen v. 20.12.1934 77, 185, $476 \mathrm{f}$., 495, 520f., 633, 661, 685, $825,833,854,951,1092,1096,1111$

Hessen 85, 98, 112, 114, 129, 240, 245, 261, 271, 301

Hildesheim $564 \mathrm{f}$.

Hilfspolizei $67,321,331,336,359 \mathrm{f} ., 370,374$, $380,386 \mathrm{ff} ., 391,393,396,433,703,831$, 1119

Hilfsrichter s. unter Richter

Hirschberg (Riesengebirge) 460, 464, 480

Hitler-Jugend $(\mathrm{HJ}) \quad 212,216,218,220,407 \mathrm{f}$., 593, 797

Hitler-Prozeß 25, 31, 33-48, 232, 236

Hitler-Putsch 20, 28, 31, 33, 35, 44, 440

Hochverrat 19f., 31,33 f., 37 f., 47, 77, 84 , $185,334,359,361,418,444,447,450$, 454 f., 458 f., $467,480,537,584$ f., 588,594 , 597, 600, 603 f., 607,613 ff., 627 ff., 631 f., 659 f., $663,703,705$ f., 710 ff., 737, 761 , 775 ff., 781, 796, 798 ff., 823 f., 826, 830, $832,837,844 \mathrm{ff}$., $862,887,889,892,906$, 916, 918 ff., 932 f., 936, 939, 943, 947, 949 , 957 ff., 964 ff., $969,977,1051$ f., 1060, 1064, $1075,1081,1091,1099 \mathrm{f} ., 1105 \mathrm{f} ., 1126$, $1132,1136,1141$

- Besprechung im Reichsjustizministerium v. 11./12.11.1936 585, 713

Hörstein (Mainfranken) 388, 393 ff., 406

Hohnstein (Sachsen) $368 \mathrm{ff}$.

Hohnstein-Prozeß 360, 369 ff., 709, 1111

Homosexuelle $76,650 \mathrm{f} ., 656,683 \mathrm{f}$., $711 \mathrm{f}$., 716, 806, 854 ff., 917, 1079

- Zahl der Verurteilten 854

I.G. Farbenindustrie 783

Indien 865

Innenminister

- badischer $615 \mathrm{ff}$.

- bayerischer $74 \mathrm{f}$., $380 \mathrm{ff}$., $388 \mathrm{ff}$., $393 \mathrm{f}$., 402, $406 \mathrm{f}$., 424, 434, 436, 550 f., 573, 586, 637 ff., $691,694,698,722,876$

- preußischer $320 \mathrm{f}$., $324 \mathrm{f}$., 328, 541, $719 \mathrm{ff}$.

- sächsischer 525

- württembergischer 874

Innenministerium

- badisches 577,580
- bayerisches $380 \mathrm{ff} ., 388,396,399 \mathrm{f} ., 404$, $408 \mathrm{ff}$., $411,548,604,610,638 \mathrm{f}$., 642, 700, 726

- oldenburgisches 539

- preußisches $157,160,222,330,346,349$, $358,362,552,555,565,571,574,612$, 698 f., $703,831,834$

- sächsisches $368,525,580$

Innsbruck 278, 285, 288, 805

Internationale Kriminalistische Vereinigung 765,864

Internationaler Gerichtshof im Haag $862 \mathrm{f}$.

Internationaler Strafrechtskongreß der Association internationale de droit pénal v. Juli 1937 in Paris 863

Internationaler Strafrechts- und Gefängniskongreß 1935 in Berlin 569, 722, 856

Italien 805, 863, 1067

Japan 865

Jena $108 \mathrm{f}$., 764, 826

Juden s. auch unter: Assessoren, Frontkämpfer, Geschworene, Halbjuden, Handelsrichter, Justizbeamte, Konkursverwalter, „Mischlinge“, Notare, Patentanwälte, Pflichtverteidiger, Rechtsanwälte, Referendare, Repetitoren, Richter, Staatsanwälte 6 , 70 f., 81 f., $127,129,132,134,140,146$, 149 f., 154 ff., 158,162 f., 165 ff., 172 f., 175 , $178,182,187,197,215,249,367,393 \mathrm{ff}$., 397 f., 403 ff., 460 f., 464,484 ff., 494 ff., 534 , $582,597,641,650,661,666 \mathrm{ff} ., 674,679$, 682 f., 695 ff., $707,725,746,761$ f., 770 f., $783,801,804,809,862,864-886,896,905$, 909, 1098, 1100, 1104, 1114, $1122 \mathrm{f} ., 1130 \mathrm{f}$., 1133,1145

- Boykott vom April 1933127 ff., 135, 157

- Pogrom vom November 1938 s. „Reichskristallnacht"

- Vertretung vor Gericht s. auch Konsulenten, jüdische $175 \mathrm{f}$., $180,183 \mathrm{ff}$., 188, 1117

Jüterbog 111, 301, $303 \mathrm{ff} ., 312,662$

Jugendarrest 910

Jugendliche 268, 854, $910 f$., 950, 960, 1053 ff., 1089, 1131, 1136

Jugendschutz 268, 782, 785, 802 f., 837, 922

Jugendstrafrecht 774, 854, $910 \mathrm{f}$., 960, $1053 \mathrm{f}$.

Jugendverbände, konfessionelle $539 \mathrm{f}$., $543 \mathrm{f}$.

Jurastudenten s. Rechtsstudenten

Juristentag

- Badischer 869

- Deutscher 68, 85, 88 760, 1084, 1139

Juristische Wochenschrift 565

Justizausbildungsordnung

- v. 22.7.1934 108 ff., 216, 267, 299 ff., 315

- v. $4.1 .1939302 \mathrm{f}$. 
Justizbeamte $672,911,974,1113$

- Altersschichtung in Bayern 238

- Ausbildung s. dort

- Beamtenuniform $291 \mathrm{f}$.

- Besoldung 97, $107 \mathrm{f} ., 112,116,118,209 \mathrm{f}$., 217, 230, 277, 280, 292-298, 971

- Dienstränge 290f., 292-298

- erhöhter strafrechtlicher Schutz $912 \mathrm{f}$.

- Ernennung s. auch Personalpolitik 112, 116,118 f., $140,154,189$ ff., 207 ff., 216 , 221 f., 230,232 ff., 238,257 ff., 262 ff., 293, 315 f., 318, 961 ff., 968 f., 1110, 1115, 1118, 1132

- „jüdisch versippte“ 172 f., 190, 251 f., 300, 972

- jüdische s. auch „Altbeamte“, jüdische 75 , $89,124 \mathrm{ff}$., $140 \mathrm{ff}$., $154 \mathrm{ff}$., $168 \mathrm{ff}$., $189 \mathrm{f}$., 223 f., $237,242,248$ f., 251 ff., 300,322 f., 1117,1144

- Laufbahnregelung 107, 119, 216, $267 \mathrm{f}$., $292 \mathrm{ff} ., 316 \mathrm{ff}$.

- national-konservative Gesinnung der höheren $288,1114,1143$

- politisch unzuverlässige $138,143 \mathrm{f} ., 154 \mathrm{ff}$., 160, 163, 165 ff., 172 f., 191, 194, 197 f., 200, $202 \mathrm{f} ., 213,224,237,659,667,670,973 \mathrm{f}$., $1114,1118,1132,1144$

- Prüfungswesen s. auch Reichs-Justizprüfungsamt $97,106,109 \mathrm{ff}$., $140 \mathrm{f}$., $300 \mathrm{ff}$.

- soziale Herkunft 266, 288 f., 1114

- weibliche $268 \mathrm{f} ., 318 \mathrm{f}$.

- weltanschauliche Schulung s. Schulung

- Zahl der 87, 119, 166, 237, $298 \mathrm{f}$.

- Zahl der Eingangs- und Beförderungsstellen $294 \mathrm{ff}$., 298, $315 \mathrm{ff}$.

- Zahl der „jüdisch versippten“ 173

- Zahl der jüdischen 129, 165 ff., 169 ff., 173

- Zwangsversetzung s. auch unter Richter $129,138,155$ ff., $166,168,222$ ff., 225,230 , 237

Justizminister

- badischer 240,327, 572

- bayerischer $23 \mathrm{ff}$, 27, 36, $47 \mathrm{ff}$., 53, 57, 64, $85,88,90,95$ f., 98 f., 102, 128 f., 230 ff., 261 , $323 \mathrm{ff}$., $334,380 \mathrm{ff} ., 388,548,637,694,698$, $769,834,961,1116$

- braunschweigischer 206, 240, 271, 326, 420

- hamburgischer (Justizsenator) 240, $273 \mathrm{f}$., 374 ff., 612, 705, 832, 949

- hessischer (Staatsministerium) 240

- mecklenburgischer $239 \mathrm{f} ., 272$

- österreichischer 1017, 1026

- oldenburgischer 239

- preußischer $64,87,95,98,100 \mathrm{ff} ., 104,127$, $147,149,155$ ff., $161,189,214,218,221$ ff., $275,299,301,315,327$ f., 330, 334, 339 ff.,
$367,447,497,587$ f., $612 \mathrm{f} ., 675,720$ ff., 754 , $757,760,1056,1116$

- sächsischer 91, $95 \mathrm{f} ., 98,141,239,246,279$, 369, 561, $758 \mathrm{f} ., 969$

- thüringischer $109,228,239,850$

- württembergischer $147,240,245,420$

Justizministerium

- anhaltisches (Staatsministerium) 344

- badisches 275, 962

- bayerisches $13 \mathrm{f}$., $18 \mathrm{ff}$., $28 \mathrm{ff}$., $34 \mathrm{f}$., 37,91 , 108, 115, 128 f., 141, 144 f., 154, 231 ff., 274, 276, 282, 299, 332, $380 \mathrm{ff} ., 392,395 \mathrm{f}$., 399 ff., 403 f., 406 f., 409 ff., 434 f., 438 ff., $458 \mathrm{f}$., 471, 477, $637 \mathrm{ff}$., 643, 719, 754, 834, 947, 961, 969, 1091, 1099

- bayerisches, Zahl der Ministerialbeamten 239

- hamburgisches $961 \mathrm{f}$.

- hessisches (Justizabteilung im Staatsministerium) $\mathbf{4 2 0}$

- preußisches 95, 99 ff., 104 ff., 119 f., 125, $132,135,140,142,153,155$ ff., 162,165 , 219,222 ff., 242 ff., $247,261,265,273,280$, 322 f., 325, 327, 331, 337, 345 ff., 354, 431, $444 \mathrm{ff} ., 453,457,474,476,539,551,564$, 574, 603, 610, 698f., 758, 760, 831, 834, 956, 961, 981, 1054f., 1109, $1116 \mathrm{f}$., 1120

- sächsisches $368,754,769,962$

- thüringisches 344

- württembergisches 273, 285, 962

Justizpressestellen 663

Justizverwaltungsrecht 92, 95, 97 ff., 102, 105, 107 ff., $116 \mathrm{f} ., 1116$

Kabinett s. Regierung

Kaiserslautern 124, 323, $383 \mathrm{f}$.

Kammergericht 86, 150 f., 167, 171, 182, 223, 259, 268, 273 ff., 283 f., 346, 534, 540, 604, $665,703,962,1100 \mathrm{f}$.

Kammergerichtspräsident $127,161,184 \mathrm{f}$., 211 f., 220, 222, 225, 281, 288, 529, 653, 671,968

Kamphausen-Prozeß 455, 465 ff., 470, 1122

Kanzelmißbrauch 77, 521, 784

Kanzlei des Führers der NSDAP 192, 219, 373, 379, $469 \mathrm{f} ., 499 \mathrm{ff} ., 506,509,511,516$, $518,520,522$ ff., $528,652,708$ f., 814, 1007, 1123

Karlsruhe 114, 740

Kassel 224, 227, 981

Kassenärztliche Vereinigung Deutschlands (KVD) 500

"Katastrophentheorie“ 2

Katholische Aktion 442

Katholizismus s. auch Kirche, katholische 21, 79, 215, 227, 242, 410, 1003, 1093 
Kiel 337 f., 769, 1015

„Kieler Schule“ s. unter Rechtswissenschaft

Kirche

- Bekennende 74, 76, 79, 227, 266, 512, 539, 807

- evangelische $74,227,256,506,513,522$, $532,539,1093,1141$

- katholische 74, $76 \mathrm{f} ., 497 \mathrm{f}$., 513, $521 \mathrm{f} ., 532$, $539 \mathrm{f} ., 698,783,867,1030,1106,1141$

Kirchenrecht $1030 \mathrm{f}$.

Klageerzwingungsverfahren 417, 424, 985, $987,1005,1012,1016,1090$

Koblenz 348, 698

Köln 287, 456, 539

Königsberg (Ostpreußen) 125, 282, 284, 324, $339,462,698,824,964$

Königssee (Oberbayern) 758

Köpenick (Berlin-) 831

Körperverletzung 341, $351 \mathrm{f}$., 387, 392, 420, $487,635,661,684,703,787,813,819,837$, 894, 917, 949, 993, 1080 f., 1096

Kommunismus 66 f., 76, 362, 465, 539, $704 \mathrm{f}$., 707, 709 ff., 781, 850, 1099

- kommunistische Betätigung 139, 142, $144 \mathrm{f}$., $149 \mathrm{f}$., $156,158 \mathrm{f}$., $166,389,392 \mathrm{f}$., $535 f$ f., $539 f$ f., $585,614,676,781,823,836$

- „mittelbare kommunistische Gefahr" 540

Kommunisten 62, 149, 327, 338 f., 356, 358, $361,384,393,460 \mathrm{f}$., 464, $535 \mathrm{f} ., 570,604 \mathrm{f}$., $615,618,631,641,709 \mathrm{ff} ., 718,731,778$, 823 f., 832, 844, 957, 964 f., 1099, 1105

Kommunistische Partei Deutschlands (KPD) 19, $51 \mathrm{f} ., 60,65,125,144,159,249,384$, $535 \mathrm{f} ., 539,585,588,707,753,957$

„Konkrete Betrachtungsweise“ 977 f., 1089

Konkursverwalter 700,703

- jüdische 128f., 140, 148, 163

Konservativismus $17,25,48 \mathrm{f}$., 52, $63 \mathrm{f}$., 66 , 79f., 156, 241, 243, 289, 765, 1114 f., 1117 , 1131,1146

Konsulenten, jüdische $81,168,176 \mathrm{f} ., 180 \mathrm{ff}$., 187,1117

- Zahl der 182

Kontumazialverfahren 1067

Konzentrationslager (KZ) 5, 76, 78, 254, 322, $345,364,366,378,429,446,478,486,505$, $521,533 \mathrm{f} ., 545 \mathrm{ff} ., 558,561,565 \mathrm{ff} ., 574$, $579,583,591,594,597,608$ ff., 614, $616 \mathrm{f}$., $623 \mathrm{f} ., 632-658,665,675,677,682,690$, $695,705,710,720,724,726,730,735$ ff., 744f., 825, 840, 854, 889, 958, 1080, 1111, $1120 \mathrm{f} ., 1124 \mathrm{ff} ., 1142$

- Auschwitz 623, 677

- Bredow 348ff., 360, 362, 368, 372, 473, 704, 706, 1120

- Buchenwald 611,651f., 690
- Columbia-Haus 322, 634, 646, $648 \mathrm{f}$.

- Dachau 183, 364, 380f., 391, 412, $437 \mathrm{f}$., $440,457,464,573,608,612,614,633 \mathrm{ff}$., $645 \mathrm{f} ., 649,652,675,684,695,698,722$, 725,825

- Emslandlager $362 \mathrm{ff}$.

- Esterwegen 362 ff., $632 \mathrm{f} ., 649,724$

- Flossenbürg 655

- Fuhlsbüttel 368, 375 f., 566, 571, 574, 578, $585,646,649$

- Hohnstein 205, 329, 335, 360, 367 ff., 374

- Kemna 335 ff., 367, 474, 704, 965

- Kislau $614 \mathrm{f}$.

- Lichtenburg 574, 576, 646, $724 \mathrm{f}$.

- Lublin 623

- Mauthausen 646,682,745

- Moringen 574, 579,608, 725

- Neusustrum 366

- Oranienburg 442, 646

- Papenburg 354

- Ravensbrück 574,619

- Sachsenburg 566, 646,649,725

- Sachsenhausen 599, 650, 653 f., 656 ff., 677, 684,725

- Wittmoor $374 \mathrm{f}$.

- rechtliche „Exterritorialität “ $632,656,658$

- Straf- und Dienstvorschriften 351, 361, 365, 637 f., 640f., 645-652, 1127

- Zuständigkeit der Justiz für Straftaten 632 ff., 653 ff., 657 f., 1127

Korruption 91, 223, 254, 287, 367, 587, $834 \mathrm{ff}$.

Kreisgericht 932 ff., 936

Kriegssonderstrafrechtsverordnung (KSSVO) v. $17.8 .1938817,901,921$

Kriegsstrafverfahrensordnung (KStVO) v. $17.8 .1938655,817,1073$

Kriminalpolizei s. auch Reichskriminalpolizeiamt $719 \mathrm{ff} ., 724 \mathrm{ff}$., $729 \mathrm{ff}$., $733 \mathrm{f}$., 740 , 742,1127

Kultusministerium, preußisches s. unter Preußen

Kurhessen (Gau der NSDAP) 484

„Ländergruppen“ der Justiz 98f., 102, 105, $107 \mathrm{ff}$.

Lageberichte

- der Generalstaatsanwälte 494, 524, 594, 686, 688, $691 \mathrm{ff} ., 1094 \mathrm{f}$.

- der Oberlandesgerichtspräsidenten $211 \mathrm{f}$., 214, 291, 311, 313 f., 494, 524, 563 f., 689, 953, 1094 f., 1106

Laienrichter s. auch Geschworene und Schöffen $37 \mathrm{ff}$., $42,371 \mathrm{f}$., $932 \mathrm{ff}$., $937 \mathrm{ff}$., $944 \mathrm{f}$., 959, 961, 967, 975 f., 982 ff., 1007 f., 1133

Landesamt für Familiengüter und Hausvermögen 106, 224, 244, 258 
Landeserbhofgericht Celle 141, 208, 270, 527

Landeshut (Schlesien) 460, 463 f., 479

Landesjustizminister s. auch unter Justizminister 4, 49, $71 \mathrm{f} ., 89 \mathrm{ff} ., 120,124,138 \mathrm{f}$, $141 \mathrm{ff} ., 145 \mathrm{ff} ., 158,163 \mathrm{f} ., 168,239 \mathrm{f} ., 344$, $834 \mathrm{ff} ., 1115,1117,1119 \mathrm{f}$.

- Konferenz im Reichsjustizministerium am 7.4.1933 136, 145f.

- Konferenz in München am 22.4.1933 $90 \mathrm{f}$., $141 \mathrm{ff} ., 146,158 \mathrm{f}$., 834

- Konferenz in Stuttgart am 6.5.1933 91, $145 \mathrm{ff}$., $163,314,821,830,835 \mathrm{f}$.

- Konferenz in Berlin am 2./3.8.1933 91, $831 \mathrm{f}$.

- Konferenz in Dresden am 12.2.1934 $96 \mathrm{ff}$.

- Konferenz der süddeutschen in München am 20.2.1934 108

Landesjustizministerien s. auch unter Justizministerium $84 \mathrm{f}$., $98 \mathrm{f}$., $107 \mathrm{f}$., $111 \mathrm{ff}$., 116 , $121,158 \mathrm{f} ., 238,241,245,270 \mathrm{f}$., $327 \mathrm{ff}$., 420 , $429,431,473,588,613,755,766,945,947$, $961,1055,1091,1099,1117$

- Verzeichnis der 95

Landesjustizverwaltungen s. Landesjustizministerien

Landeskriminalpolizeiamt, preußisches s. unter Preußen

Landesregierungen s. auch unter Regierung $68,329,539,547 \mathrm{f} ., 550,561,700,869$

Landesverrat $25 \mathrm{ff} ., 77,84,185,204,334,359$, 418, 450, 458f., 480, 513, 521, 537, 572, 584, 588, 597, 612f., 617 ff., 627 f., 631, 687, $714,716,737,761,771,776,778,781,794$, 796, 798 ff., 807, 812, 824, 837, 844ff., 862, $888 \mathrm{f} ., 892,900,910,916,918,920,923$, $932 \mathrm{f} ., 936,939,943,947,949,958 \mathrm{ff}$. , 964 ff., 969, 1051f., 1060, $1071 \mathrm{f} ., 1075 \mathrm{f}$, $1091,1095,1126,1132,1136,1140 \mathrm{f}$.

Landfriedensbruch 29, 31, 337, 404, 491, 813

Landgericht(e) $293 \mathrm{ff} ., 317,582,878,932 \mathrm{ff}$., $938 \mathrm{ff} ., 947 \mathrm{f} ., 951,953,968,971 \mathrm{ff} ., 975 \mathrm{ff}$., 989, 991, 1015, 1033, 1039, 1057, 1061, 1069 f., 1079, 1084, 1098, 1101, 1132, 1134, $1136 \mathrm{f}$.

- Aachen 424

- Altona 157, 338

- Amberg 236, 636, 861

- Ansbach 404f.

- Aschaffenburg 395

- Augsburg 259, 400

- Aurich 532

- Bamberg 181, 215, 237, 949

- Bayreuth 386

- Berlin 126f., 223, 225, 229, $281 \mathrm{ff}, 298$, 442, 460, 463f., 482 f., 534, 540, 656, 665, $703,848,899,972$
- Beuthen 225, 326

- Bonn 214, 229, 279, 540, 896

- Braunschweig 461, 463

- Breslau 322, 461 ff., 465 ff., 469

- Brünn 279

- Celle 213

- Chemnitz 124, 705

- Dachau 641

- Danzig 280

- Dortmund 953

- Dresden 329, 368 ff., 1111

- Düsseldorf 509

- Duisburg 275

- Eger 286

- Elbing 275

- Flensburg 157, 286, 337f., 340, 343, 349, 353

- Frankenthal 282

- Frankfurt a.M. 213, 502, 534

- Glatz 280

- Graz 278

- Greifswald 281

- Guben 228

- Halberstadt 275

- Halle 283, 705

- Hamburg 309, 374f., 718, 730, 732f., 879, 935, 953, 981, 1106

- Hamm 287

- Hanau 876

- Hannover 280, 459, 468, 495, 674, 1111

- Heidelberg 740

- Hirschberg 460

- Hof 236

- Kaiserslautern 236, 323, 383

- Karlsruhe $881 \mathrm{f}$.

- Kassel 229, 347, 461, 478, 482

- Kempten 285

- Kiel 228

- Klagenfurt 596

- Koblenz 214, 220, 495

- Köln 220, 229, 283, 899, 1028

- Königsberg 284, 872

- Krems 582

- Landsberg (Warthe) 305

- Landshut 382, 494, 575

- Leipzig 126, 517, 665

- Liegnitz 1109

- Limburg 272, $529 f$.

- Lüneburg 674

- Lyck 228, 275

- Mannheim 319

- Marburg 227

- Memel 908

- Meseritz 275

- München I 13, 16, 18, 20f., 29, 35, 38, 42 ff., 48, 232, 235 f., 276 f., 378, 384, 406, 
435, 437, 439 ff., 447, 459, 462, 464, 494, $606,633,684,686,695,718,961,1080$

- München II 18, 412, $438 \mathrm{ff} ., 442,459$, $634 \mathrm{ff} ., 642 \mathrm{f}$.

- Münster 515, 519

- Naumburg 683

- Neiße $192 \mathrm{ff}$.

- Nordhausen 685

- Nürnberg-Fürth 285, $386 \mathrm{f}$.

- Oldenburg 279

- Osnabrück 363 ff., 459f., 463, 468, 478, 532

- Passau 464

- Potsdam 229, 443

- Prag 279

- Prenzlau 379

- Regensburg 12, 381

- Reichenberg 278

- Schneidemühl 184, 275

- Schweinfurt 181, 232, 460, 464

- Schwerin 709

- Stendal 1081

- Stettin 349 ff., 407

- Steyr 286

- Stuttgart 273

- Traunstein 479

- Tübingen 544

- Verden 674

- Waldshut 287

- Weimar 660

- Wels 520, 523

- Wien 278

- Wiesbaden 525

- Würzburg 181, 276

- Wuppertal 354, 356, 359, 362, $733 \mathrm{f}$.

- Zwickau 279, 282

Landgerichtspräsident(en) 121, $149 \mathrm{f} ., 153$, $167,182,193,202,210,214,217,225,237$, 239, 263, 289, 291, 294, 379, 494, 516, 530 ff., 714, 730, 732, 934, 936, 946, 953, 968, 971, $973 \mathrm{f}$., 1015, 1028, 1098, $1105 \mathrm{f}$., 1109

Landsberg (Warthe) 512

Landshut (Niederbayern) 382, 494

Landshuter Zeitung 382

Landtag

- bayerischer 14, 23, 25 f., 29 f., 36,38 f., 41, 46,48 ff., $54 \mathrm{f} ., 71,85$

- preußischer 60, 64, 87, 224, 266, 962

Lauf (Pegnitz) 532

Laufbahnregelung $s$. unter Justizbeamte

Legalitätsprinzip $417,419,519,638,791$, $808 \mathrm{f} ., 813,987,1012,1047,1064 \mathrm{f} ., 1090$, $1107 \mathrm{f}$., $1121 \mathrm{f} ., 1135$

Leipzig 33 f., 125 f., 501, 754, 769, 960 f., 1078 , 1081

Leipziger Neueste Nachrichten 529
Lengermoos (bei Dachau) 438

Lenkung der Rechtsprechung 6, 454, 621, $623,659,670,729$ ff., $736,740,742$ ff., 839 , 879, 905, 954f., 1035, 1091-1112, 1115, $1138 \mathrm{ff}$.

- durch veröffentlichte Urteilsbesprechungen 539, 541 f., 861, 927, 965, 1095, 1099, 1141

- Einwirkung auf die Richter 1095, 1097 ff., $1105 \mathrm{ff} ., 1141 \mathrm{f}$.

- „Nachschau“ 1142

- „Richterbriefe“ 1105, 1141

- Tagungen im Reichsjustizministerium 1099 f., 1108,1141

- über den Staatsanwalt 540, 659, 729, $742 \mathrm{ff}$., 978, $1081 \mathrm{f} ., 1087 \mathrm{ff} ., 1095,1097 \mathrm{ff} ., 1105 \mathrm{ff}$., $1111,1141 \mathrm{f}$.

- „Vorschau“ $1109 \mathrm{f} ., 1142$

Leobschütz (Oberschlesien) 459

Leoprechting-Prozeß 38

Lesum (Bremen-) 492

Lex Giese s. Gesetz gegen erpresserischen Kindesraub v. 22.6.1936

Lex Höfle s. Gesetz zur Abänderung der Strafprozeßordnung v. 27.12.1926

Lex Kompalla s. Gesetz zur Änderung von Vorschriften des allgemeinen Strafverfahrens, des Wehrmachtstrafverfahrens und des Strafgesetzbuchs v. 16.9.1939

Lex van der Lubbe s. Gesetz über Verhängung und Vollzug der Todesstrafe v. 29.3.1933

Lichterfelde (Berlin-) 442, 459

Liga für Menschenrechte 138, 249

Limburg (Lahn) 522

Linz (Donau) 805

Lippe 112, 114

Lothringen, Chef der Zivilverwaltung 283

Lübeck 98, 107, 112, 114

Lütjensee (Schleswig-Holstein) 301, 304

Luftschutzgesetz 1088, 1093

„Lycker Affäre“ 228, 275

Märkische Film-GmbH 499

Magdeburg 541

Magdeburg-Anhalt (Gau der NSDAP) 484

Mannheim 147f., 287

Mariental (Heilanstalt) 519

Marxismus, Marxisten s. Kommunismus, Kommunisten

Maßregeln der Sicherung und Besserung 726, 736, 741, 743 f., 774, 777, 789, 840 ff., 851 , 942, 956, 977 f., 992, 1026 ff., 1058 f., 1063 , 1074, $1134 \mathrm{f}$.

- Zahlen 844

Maximilianeum München 11f., 21, 75

Mecklenburg 85, 112, 239, 245, 273

„Mein Kampf“ 746, 881, 956 
Meineid 711, 785, 799, 852, 883, 885, 939, 949, 1014, 1053, 1089

- Zahl der Verurteilten 1053

Meyen (Kreis Koblenz) 348

Miesbacher Anzeiger 445

Mieterschutz für Juden 668

Militärgerichtsbarkeit s. auch Wehrmachtgerichtsbarkeit 1, 19, 414 ff., 420, 422, 813, $817,900,910,915,921,937,944,960$, 1012

Militärstrafgerichtsordnung 196, 417 ff., $654 \mathrm{f}$., $960,1015,1073$

Militärstrafgesetzbuch 415, 417, 428, 466, $470,805,807,813,817$ f., 855, 920, 960 , 1072

Militärstrafrecht $\mathbf{8 0 5 , 8 8 7 , 9 0 7}$

Militärstrafverfahren 1072 ff., 1076

Ministerialbürokratie $65,121,168,190,258$, $746,749,752,765,874,876,1115,1117$

Ministerpräsident

- bayerischer 230, 232, 234 f., 333, 380 f., 389, $397,421,435,441,550,637 \mathrm{ff}$.

- preußischer s. auch im Personenregister unter Göring 103, 120, $122 \mathrm{f}$., 157, 200, $221 \mathrm{ff}$., $331,334,341,358,445,547,551 \mathrm{ff} ., 555 \mathrm{ff}$., 564, 569 ff., 603, 614, 675, 699 f., 835

- sächsischer 369

Ministerrat für die Reichsverteidigung 597, $687,689,819$ ff., 902 f., 906, 911 f., 916, $919 f ., 922,944$

„Mischehe(n)“ 172, 761, 864 ff., 871 ff., 1130

"Mischlinge“, jüdische $168 \mathrm{f}$., $173 \mathrm{f}$., 177, 180, 188 f., 252 ff., 866, 868, 874, 876 ff., 1117

"Mitteilungen in Strafsachen" 356, 408, 488, $519,729,861,1061,1092$

"Mitteilungen in Vollzugssachen“ 623, 729

"Möttlinger Bewegung“ 666, $669 \mathrm{ff}$.

Monbachtal (Württemberg) 305

Mondorf (Luxemburg) 436

„Monstrestrafsachen“ $932 \mathrm{f} ., 936,982$

Moringen, Frauenschutzhaftlager s. unter Konzentrationslager

München 11, 14, 16, 18, 20, $33 \mathrm{f} ., 83,90$, $109 \mathrm{ff} ., 114,179,183,325,380,383,413$, $434 \mathrm{ff} ., 447,462,485 \mathrm{f} ., 488,513,634 \mathrm{ff}$., $694,698,754,757,794,930,1002,1078$

München-Augsburger Abendzeitung 53, 55

„Münchener Post“ $53 \mathrm{f}$.

Münchner Neueste Nachrichten 75, 440, 684, $686,894,903,951,965$

Münchner Zeitung 54, 473

Münster (Westfalen) 513, 519, 521

\section{Nachprüfung}

- gerichtliche $537 \mathrm{ff} ., 543 \mathrm{f} ., 546,556,558$, $562,564,576,599,714,721,739,823,935$,
986, 988, 1012, 1014, 1016, $1023 \mathrm{f}$., $1033 \mathrm{f}$., $1060,1066,1071$ ff., 1076, 1082 f., 1085 , $1124 \mathrm{f} ., 1127,1135,1144$

- gerichtlicher Urteile durch eine politische Instanz 997 f., 1007 ff., 1046, 1048 f., 1134, 1140

„Nachschau“ s. unter Lenkung der Rechtsprechung

„Nacht-und-Nebel“-Verfahren 264, 1043 , 1145

Nachwuchs, juristischer 110, 114, 216f., $237 \mathrm{f}$., $270,298 \mathrm{ff}$., $311 \mathrm{ff} ., 1137$

Nachwuchsprobleme 5, $268 \mathrm{f}$., $312 \mathrm{ff} ., 318 \mathrm{f}$.

Nationale Front 64, 1113

Nationalsozialistische Deutsche Arbeiterpartei (NSDAP) 5, 16, 51 ff., 58 ff., 62 ff., 66 f., 80, 83, 87, 89, 102 f., $114 \mathrm{f} ., 117,121,123,133$, $145,149,153,155,157,168,174$ ff., 185 , 188,190 f., 202 f., 215 ff., 222 ff., 232 f., 242 , 248, 261, $271 \mathrm{ff}$., 291, $300 \mathrm{ff} ., 307,311 \mathrm{f}$, $319 \mathrm{ff}$., $345,355 \mathrm{ff} ., 367,371,377 \mathrm{f}$., $380 \mathrm{ff}$., 388, 394, 397, 407 f., 410,421 ff., 428, 437, $446,464,473$ f., 477 f., 484 ff., 496,499 ff., $572,582,584,633,662,667,670$ f., 680 , $694 \mathrm{f} ., 746 \mathrm{ff} ., 753 \mathrm{f} ., 764,767 \mathrm{ff} ., 773,784 \mathrm{f}$., $790 \mathrm{f}$., $795,797 \mathrm{ff}$., $801,804,806 \mathrm{ff}$., $813 \mathrm{ff}$., $821,825,832$ f., 848,852 ff., 863 f., 866 , 869 ff., 874 ff., $885,889,903,911,920 \mathrm{ff}$., 950, 954, 957, 961, 966, 969, 980, $999 \mathrm{ff}$. ., 1006 f., 1011, 1015, 1045 ff., 1092, 1096, 1102 , 1111, 1113 ff., 1117, $1119,1122 \mathrm{f}$., $1129,1135,1141,1144$

- Altparteigenossen $212 \mathrm{ff}$., 218, 222, $225 \mathrm{f}$, 232, 234, 240, 244 f., 261, 265 f., 269 f., 271, 276, 279, 282 ff., 353 f., 694, 962, 969

- Amt für Rechtspolitik, bis 1935 s. unter NSDAP, rechtspolitische Abteilung der Reichsleitung 747, 795, 993

- Amtsträger 797, 832, 890, 916, 949

- Beamteneigenschaft der Amtsträger 890

- Beteiligung eines Kommissars am Strafverfahren $999 \mathrm{f}$.

- Dienststellen der 77, 156, 195, 201 f., 205, 210, 272, 275, 408, 439, 441, 477, 485, 487, $489,529,549,586,697,747,795,809,814$, $816,852,866,871,874,903,937,948$, $1014,1047 \mathrm{f} ., 1111,1116,1119 \mathrm{ff} ., 1132$, $1141 \mathrm{f}$.

- Eingriffe in die Justiz $77,121,126 \mathrm{f} ., 254$, 272, 322 ff., 330, 332, 336 ff., $341 \mathrm{f}$., 348 , $355,357,359,370$ ff., 380 ff., 397 ff., 401 , $420,428,472$ f., 488 f., 491, 582, 694 ff., 832 , $871,999,1014,1068,1111,1116,1119 \mathrm{ff}$., 1142

- Gauleiter s. dort

- Gaurechtsstellen 128, 431, 965 
- Gliederungen und angeschlossene Verbände 797, 810, 815f., 890, 1124

- Goldenes Parteiabzeichen 80, 219, 271, 276, 353, 965

- Hauptamt für Beamte 217

- Hauptamt für Volkswohlfahrt 797

- Hauptamt für Weltanschauliche Information beim Beauftragten des Führers für die Überwachung der gesamten geistigen und weltanschaulichen Erziehung 513

- Informationspflicht der Justizbeamten gegenüber der Partei $204 \mathrm{f}$.

- Leitsätze des Reichsrechtsamts für ein neues Strafrecht 768,775

- Mitgliedersperre 217, 220, 225, 262, 265, 963

- Mitgliedschaft von Justizbeamten 191, 203 ff., 208 f., 214,216 ff., 225 f., 232 f., 239 , 241 ff., $250 f$., 259 ff., 269-288, 300, 372, $670,940,948,954,962$ f., 969, 1002, 1113, 1132

- Mitteilungspflicht der Staatsanwälte $356 \mathrm{f}$., 430 f., 1093

- Mitwirkung bei der Berufung von Schöffen und Geschworenen 934, 936, $945 \mathrm{f}$.

- Mitwirkung bei der Gesetzgebung $746 \mathrm{ff}$., 767 ff., 794 ff., 852 f., 874 f., 890, 903, 920 ff., $1011,1015,1046,1115,1129,1135$

- Mitwirkung bei der Personalpolitik 121, $156 \mathrm{f}$., $202 \mathrm{ff}$., $207 \mathrm{ff}$., $253,256 \mathrm{ff}$., $262 \mathrm{ff}$., 269 , 276 f., 814, 948, 962 ff., 1111,1116 ff., 1132, 1138,1142

- Parteigerichte s. dort

- Parteiprogramm 215, 218, 746, 782, 859, $864,866,868,870,876,926,1139$

- Parteitage s. dort

- politische Beurteilung von Justizbeamten 205, 207 ff., $214 \mathrm{f}$., 276, 281, 302, 1118, 1142

- Problem der Loyalität beamteter Parteigenossen $203 \mathrm{ff} ., 372,377$

- Rechtsabteilung der Reichsleitung, ab 1935 s. unter NSDAP, Reichsrechtsamt 86, 115, $126,141,143,234,747,754$ f., 757, 1116

- rechtspolitische Abteilung der Reichsleitung, ab 1935 s. unter NSDAP, Amt für Rechtspolitik 141, 747, 757

- Reichsrechtsamt, bis 1935 s. unter NSDAP, Rechtsabteilung der Reichsleitung 10, 68, $89,180,210,308,567,747,766,768,770$, 795 f., 818, 852, 872, 875, 993 f., 1077

- Reichsschatzmeister 219, 233, 504, 833

- Strafrechtsausschuß des Reichsrechtsamts $767 \mathrm{f}$., 773

- Vernehmung von Angehörigen 1068, 1136

- Verstöße von Mitgliedern gegen die Strafgesetze $94,274,320 \mathrm{ff} ., 328 \mathrm{ff}$., $337,348 \mathrm{f}$.,
354 f., 366 f., 485 ff., 493 f., 584, 593, 595, 791, 797, 808 ff., 813 ff., 874 f., 877, 1046 ff., $1087,1093,1116,1119$ ff., 1123, 1129, 1144

Nationalsozialistische Frauenschaft 73,218 , 268, 797

Nationalsozialistische Kriegsopferversorgung (NSKOV) 251, 797

"Nationalsozialistische Parteikorrespondenz “ 738, 957

Nationalsozialistische Volkswohlfahrt (NSV) 193, 219, 251, 797

Nationalsozialistischer Bund Deutscher Technik (NSBDT) 797

Nationalsozialistischer Deutscher Ärztebund 503, 797

Nationalsozialistischer Deutscher Dozentenbund 218

Nationalsozialistischer Deutscher Reichskriegerbund 251

Nationalsozialistischer Deutscher Studentenbund (NSDStB) 218, 797

Nationalsozialistischer Lehrerbund (NSLB) 797

Nationalsozialistischer Rechtswahrerbund (NSRB) s. auch Bund Nationalsozialistischer Deutscher Juristen (BNSDJ) 175 ff., $182 \mathrm{f}$., 188, 193, 210, 214, 217, 266, 312, 684, 795, 797, 935, 996, 1002 f., 1006, 1008 ff., 1017, $1036,1038,1045,1056,1070,1117,1134$, 1139

- Ausschuß für Strafverfahrensrecht, Gerichtsverfassung und Strafvollzug 938, 994, $1002 \mathrm{f} ., 1005,1009,1011,1015 \mathrm{f}$.

- Denkschrift v. 30.1.1937 zum StVO-Entwurf des RJM 938, 1003 ff., 1045, 1134, 1140

- Stellungnahme der Wissenschaftlichen Abteilung vom September 1936 zum StVOEntwurf des RJM $995 \mathrm{ff} ., 1004$ ff., 1018

- Wissenschaftliche Abteilung $994 \mathrm{ff}$.

- Zahl der Mitglieder 221

Nationalsozialistisches Fliegerkorps (NSFK) 219f., 242, 251, 799, 806, 832

Nationalsozialistisches Kraftfahrkorps (NSKK) 216, 218 ff., 250, 269, 797

„Nationalsozialistisches Strafrecht“ (Denkschrift des Preußischen Justizministeriums) 223, 497 f., 760 ff., 768,770 f., 775,777 f., 781, 783 f., 786, 788, 801, 807, 849, 864 ff., 871, 1056

„Negative Auslese“ 745, 1103

Neisse (Oberschlesien) 734

Neubabelsberg (bei Potsdam) 443

Neues Tageblatt 465

Neumarkt (Oberpfalz) 958

Neuruppin (Heilanstalt) 512 
New York 661

Nichtigkeitsbeschwerde 6, 944, 954, 1037, 1040, 1081-1088, 1109, 1135, 1137

- Handhabung $1085 \mathrm{f}$.

- im österreichischen Recht $1084 \mathrm{f}$.

- Zahl der Fälle 1085 ff., 1137

„Der Niederbayerische Bauer" 382

Niederbayern 825

Niederschlagung 22, 93, 224, 254, 324, $327 \mathrm{f}$., 329-336, 342, 347, 353, 360 ff., $366 \mathrm{f}$., 369, $372 \mathrm{f}$., $385,390,392 \mathrm{f}$., 398, 402, $411,428 \mathrm{ff}$., $448,451 \mathrm{f}$., 460, 464, 470, 475, 480, $487 \mathrm{ff}$., 492 f., $501,639,642,644$ f., 704,708 f., 712 , $715 \mathrm{f}$., $808 \mathrm{f}$., $813,816,835,1046 \mathrm{f}$., $1080 \mathrm{f}$., $1119,1121 \mathrm{f}$., 1127

Nötigung $330,337,813,842,854,917,1064$, 1080

Notare s. auch Reichsnotarkammer und Reichsnotarordnung 88, 97, 119, 121, 137, 140 f., $229 f$., 237, 266, 289, 299, 317, 565, 700 f., 703, 1093

- jüdische $128 \mathrm{f}$., 132, 140,147, 150, $152 \mathrm{f}$., $174 \mathrm{f}$.

- Zahl der 152, 175

- Zahl der jüdischen $152 \mathrm{f}$., 175

Notverordnung

- bayerische vom 24.7.1922 23

- bayerische vom 26.8.1931 $54 \mathrm{f}$.

Notverordnungsrecht s. unter Reichspräsident

Nürnberg 179, $386 \mathrm{ff} ., 641,754,870,875 \mathrm{f}$., 958, 1130

Nürnberger Gesetze s. Reichsbürgergesetz, Reichsflaggengesetz und Blutschutzgesetz

nulla poena sine lege $763 \mathrm{f}$., $826,849,851$, 853, 863, $1128 \mathrm{f} ., 1144$

nullum crimen sine poena $849,851,1129$

Oberbefehlshaber der Luftwaffe 818, 922

Oberhausen (Rheinland) 587

Oberhof 766

Oberkommando der Wehrmacht (OKW) 199 , 259, 654, 678, 805, $817 \mathrm{f}$., 821, 901, $910 \mathrm{f}$., 920, 1070 ff., 1074 f., 1078, 1082

Oberkommando des Heeres (OKH) 654, 677 f., 915,920

Oberlandesgericht(e) $96,113,166 \mathrm{ff}$., 208, 220 , $290,293,295,300,317,347,408,496,588$, $614,618,620,630,713,729,859,932 \mathrm{ff}$., 936, 938 ff., 943 f., 946 f., 949,951 ff., 960 f., 968, 972 f., 975 ff., 979, 982, 988 f., 991, $1037,1039,1041,1044,1051 \mathrm{f} ., 1061 \mathrm{f}$. , $1064,1069,1075 \mathrm{f} ., 1081,1083,1085 \mathrm{f}$., $1094 \mathrm{f} ., 1100,1133,1137$

- Bamberg 2, 150, 167, 171, $181 \mathrm{f} ., 211,214$, 237, 276, 285, 310, 738, 1098

- Berlin s. Kammergericht
- Braunschweig 150, 167, 171, 271, 298

- Breslau 150 f., 167, 170f., 182, 228, 274, 287, 328, 621, 792, 1109

- Celle 87, 150f., 167, 171, 182, 214, 220, 225, 279, 364, 621, 674

- Danzig 275, 280, 287, 298

- Darmstadt 150, 167, 171, 182, 259, 298, 621

- Dresden 150, 167, 171, 182, 621, 705, 947

- Düsseldorf 150f., 167, 171, 182, 261, 328, 621,962

- Frankfurt a.M. 150f., 167, 171, 182, 279, 316

- Graz 278, 298

- Hamburg („Hanseatisches Oberlandesgericht $^{*}$ ) 2, 150, $167 \mathrm{f}$., 171, 182, 220, 239, $311,531,571$ f., 604, 623, 627, 660, 663, $669,672,705,712,1102,1106 \mathrm{f}$.

- Hamm 150f., 167, 171, 182, 225, 604, 715

- Innsbruck 278, 298

- Jena 109, 114, 150, 167, 171, 182, 621

- Karlsruhe 150, 167, 171, 182, 738, 869

- Kassel 150f., 167, 171, 182, 604

- Kattowitz 298

- Kiel 150f., 156, 167, 171, 182, 337, 849

- Köln 117f., 150f., 167, 171, 182, 230, 283, 299, 317, 379

- Königsberg 150 f., 167, 171, 182, 275, 298, 621

- Leitmeritz 278, 286, 298

- Linz 278, 286, 298

- Marienwerder 150f., 167, 171, 176, 275f., 298, 316

- München 35f., 119, 150, 167, 171, $182 \mathrm{f}$., $232,235,237,239,491,540,544,607,625$, $669,769,885,935,961,1102$

- Naumburg a.S. 150 f., $167,171,182,284$, $588,596,621,705,947$

- Nürnberg 150, 167, 171, 182, 239, 282

- Oldenburg 150, 167, 171, 738

- Posen 280, 298 f., 317

- Prag 279, 287, 298

- Rostock 150, 167, 171, 182, $272 \mathrm{f}$.

- Stettin 150 f., 167, 171, 182, 215, 227, 275, $281,287,621$

- Stuttgart 150,167, 171, 182, $304 \mathrm{f}$.

- Wien 174f., 177, 277 f., 298, 581

- Zweibrücken 117, 150, 167, 171, 182, 232, 282, 299, 491, 621, 947

- Zahl der 298, 939

Oberlandesgerichtsbezirke s. Oberlandesgerichte

Oberlandesgerichtspräsident(en) $96 \mathrm{f}$., $112 \mathrm{f}$,, $119,121,127$ ff., $135,144,149,153,155$, 162, 166, 170, 172, 181 ff., 193, 203, 208, $211,217,222,225,229,236$ f., 239, 263, 
266, 269 ff., 288 ff., 294, 301 f., 307,318 , $330,401,514,516,518,523$ f., 530, $572 \mathrm{f}$, $587,623,625,670 \mathrm{ff}$., $688 \mathrm{f}$., $700 \mathrm{f}$., 713 , $744 \mathrm{f} ., 791$ f., 878f., 885, 905, 909, 934, $936 \mathrm{f} ., \quad 953 \mathrm{f} ., 972 \mathrm{ff} ., 1015,1034,1037$, $1056 \mathrm{f}$., 1093 f., $1097 \mathrm{ff} ., 1105,1118,1123$, 1141

- Bamberg 213, 230f., 236, 276f., 313, 688, 694

- Braunschweig 206, 271, 291, 313, 599, 688, 694, 713

- Breslau 125, 161, 185, 193, 225, 274, 282, 322, 463, 792, 1056, 1107, 1109

- Celle 176, 220, 225, 281, 532, 1056

- Danzig 280

- Darmstadt 240, 271f., 291, 313 f., 522, 530 , $575 f$., $601,686,688,744$

- Dresden 185, 211, 220, 239, 279, 529, 1056

- Düsseldorf 220, $224 \mathrm{f} ., 288,307,313,521$, 530 f., 694, 714, 792

- Frankfurt a.M. 161, 185, 214, 220, 225, 279, 307, 515, 524f., 529, 599f., 664, 694

- Graz 278, 581, 610

- Hamburg 168, 220, 273 f., 309, 311, 313, $379,488,531,563,585,600,623$ ff., 667 , $705,712,718,727,730,733,974,1056$, 1106

- Hamm 176, 202, 211, 220, 225, 283, 515, $591,689,792,953,1056,1106$

- Innsbruck 278, 581, 610

- Jena 239

- Karlsruhe 213, 240, 275, 319, 671, 1056 , 1097

- Kassel 225, 229, 313, 694, 1097

- Kiel 225, 313, 338 ff., 413, 671, 694, 1026, 1028, 1035, 1042

- Köln 220, 225, 229, 306, 313, 495, $529 \mathrm{ff}$., $689,1056,1097$

- Königsberg 225, 228, 275 f., 281, 284, 313, $515,694,1056$

- Leitmeritz 82, $277 \mathrm{f}$.

- Linz 278, 610

- Marienwerder 202, 225, 275 f., 281

- München 37f., 220, 236, 274, 307, 477, 494, 689, 885, 958, 1056, 1097

- Naumburg a.S. 224 f., 229, 588, 613, 685, 792

- Nürnberg 214, 220, 230, 236, 274, 285, 689,694

- Oldenburg 239, 279

- Posen 280

- Prag 279f., 287

- Rostock 239, $272 \mathrm{f}$.

- Stettin 184, 212, 215, 225, 227

- Stuttgart 240, 273, 517, 1056, 1097

- Wien $277 \mathrm{f}$., 288, 516, 581, 610
- Zweibrücken 232, 236, 279, 323, $688 \mathrm{f}$.

- Berichte an das Reichsjustizministerium s. Lageberichte

- Tagung der bayerischen v. 31.3.33 231

- Tagung der bayerischen v. 5./6.4.1934 399, 407 f., 427 ff., 1099

- Tagung der bayerischen v. 7./8.12.1934 411, 477, 1099

- Tagung der preußischen v. 22.7.1933 1099

- Tagung v. 3.4.1935 791

- Tagung v. $13 . / 14.11 .1936664,881,1100 \mathrm{ff}$.

- Tagung v. 18.6.1937 213, 216f., 313, 563, $601,620,623,1100,1102$

- Tagung v. 17./18.1.1938 192, 311, 974, 1102

- Tagung v. 24./25.1.1939 $490 \mathrm{f} ., 591 \mathrm{f}$., 600 f., 624 ff., 667,669 ff., 885, 1102

- Tagung v. 27.2.1939 211, 213

- Tagung v. 23./24.4.1941 312 f., 527 ff., 531, 598,1123

- Tagung v. 31.3.1942 216

- Tagung v. 29.9.1942 1139

- Tagung v. 10./11.2.1943 292, 692

Oberpfalz 825

Oberprisenhof 265

Oberreichsanwalt

- beim Reichsgericht 24, 34f., 121, 208, 217, $240,263,283,291,294,449,527,577,593$, 627, 684, 744f., 848, 879, 882 f., 905, 914, 943, 949, 957, 960,964, 968, $1037 \mathrm{ff}$, 1051 f., 1072, 1074 f., 1077 ff., 1082 ff., 1094, $1099 \mathrm{f} ., 1110,1136 \mathrm{f}$.

- beim Reichsgericht, Bestimmung der Zuständigkeit des Besonderen Strafsenats des Reichsgerichts $1041 \mathrm{ff} ., 1076,1081$

- beim Volksgerichtshof 217, 263, 287, 291, 294, 527, 596f., 602f., 614, 618, 620, 629, $744 \mathrm{f} ., 905,914,919,943,971,1075$, 1077 ff., 1083, 1136

Oberreichskriegsanwalt 71

Obersalzberg s. Berchtesgaden

Oberschlesien 170, 225, 266, 878

Obersleben (bei Weimar) 679

Oberstaatsanwalt s. Staatsanwaltschaft

Oberste SA-Führung (OSAF) 246, 329, 331, $339,341,354,364,371,381 \mathrm{f}$., 384, 389, $396 f$., 399 f., 403 f., 412 ff., 424 f., 427, 457, 477,766

Oberster Gerichtsherr (Hitler) 423, 453, 458, $500,997,1003,1048,1072,1075$ ff., 1134

"Oberster Richter“ (Hitler) 194, 453 f., 1076, 1140

Oberstes Fideikommißgericht 270

Oberstes Parteigericht 205, $357 \mathrm{ff} ., 371,377 \mathrm{f}$., 422, 485 f., 488 f., 492, 532, 993, 1122 
Oberverwaltungsgericht, preußisches $537 \mathrm{f}$., $542,556 \mathrm{f}$.

Öffentliches Recht $86,106,242,244,262,549$

Öffentlichkeit

- Ausschluß der 35, 369, 389, 393, 404, 419, 714, 934, 937, 956, 983

- Begriff der 1111

- Tatbestandsmerkmal 901

Oels (Schlesien) 125, 459 f., 463

Österreich 37, 173 ff., 177 f., $180,182,188$, $196,198,221,260,264,268,277$ f., 285 f., 288, 303, 312, 529, 581 f., 596, 805, 807 f., 899, 905, 929, 1025 f., 1084 f., 1100

- Oberster Gerichtshof in Wien 174, 277, 1084

Oldenburg 112, 114, 239, 279

Olympiade in Berlin 1106

Opportunitätsprinzip 1058

Ordnungsstrafrecht 780, 793, 1008

Osnabrück $363 \mathrm{ff}$., 522

Ostgebiete, eingegliederte $689,694,741 \mathrm{f}$., 915,1145

Ostpreußen 275, 284, 326, 339

Palästina $15 \mathrm{f} ., 57$

Parlamentarismus $16 \mathrm{f} ., 24 \mathrm{f}$., 32, $49 \mathrm{f} ., 55 \mathrm{ff}$., 65f., 68, 822, 1113, 1115, 1128, 1143, 1146

Partei s. NSDAP

„Parteibuchbeamte“ 137, 155 f., 166

Parteien 50, 62, 65, 68, 143 f., 154, $156 \mathrm{f}$., 159, $209,249,320,322,329,863,1113,1115$, 1143

- Oppositionsparteien in Danzig 863

- Pluralismus der 13, 57 f., 66, 822, 1114, 1128,1143

Parteigerichte der NSDAP s. auch Oberstes Parteigericht 205f., 209, 227, 276, $356 \mathrm{ff}$., $361,371,402,421$ ff., 487 ff., $670,812,816$, 1009, 1048f., 1093, 1122

- Denkschrift Hitlers vom September 1933 421 ff., 1049

Partei-Kanzlei 115, 173, 203, 246, 521, 751

Parteitage in Nürnberg $81,168,178 \mathrm{f} ., 201$, 212, 258, 388 f., 392 f., 464, 500, 558, 875 , 994, 1130

Pasing (München-) 640

Patentanwälte $158 \mathrm{f} ., 163,187$

- jüdische 139, $187 \mathrm{f}$.

- Versorgung der jüdischen $187 \mathrm{f}$.

Patentanwaltskammer 88

Patentrecht s. auch Gesetz, Patentgesetz 783, 787, 927, 1132

Personalpolitik 5, 26, 50, 71, 94, $113 \mathrm{f} ., 121$, 124 ff., 137, 161, 191, 207 ff., 210 ff., 221 bis 240, 241-289, 316, 670, $960 \mathrm{ff} ., 965 \mathrm{f}$., 1115 ff., 1132,1138
Personalreferenten $232 \mathrm{f}$., $249 \mathrm{ff}$., $255 \mathrm{f}$., 259 , 265, 962, 972

Personenstandsrecht 803 f., 871, 1093

„Petit Marseillais“ 482

Plau (Mecklenburg) 770

Plünderung 485, 487, 491, 907, 952, 1093 , 1103,1130

Pösing (Oberpfalz) 381

Polen s. auch Ostgebiete, eingegliederte und Generalgouvernement 915, 1073

- s. auch Zivilarbeiter, polnische 82, 583, 689 ff., 905 f., 915,1145

- Todesstrafe für Geschlechtsverkehr mit Deutschen $689 \mathrm{ff} ., 1127$

Polenstrafrecht $691 \mathrm{f} ., 915,1127$

„Politiken“ 705

Politische Strafsachen s. Tatbestand, politischer

„Politisierung“ der Justiz 50, 670, 1114

Polizei 3, 32 f., 39, 59 f., 62, 67, 78, 149, $184 \mathrm{f}$., 224, 233, 280, 291, 313, 320 ff., 337, 345, $363,397,404$ ff., 410,434 f., 442 f., 464 , 478 f., 485 ff., 534 ff., 553, 574, 583 ff., 678 f., 719 ff., $796,825,831,837,840,842,844$, $854,874,883,886,910,949,956,980,995$, 997, 1001, 1012, 1018-1032, 1044, 1047, $1055,1058,1080 \mathrm{f} ., 1114,1124 \mathrm{ff} ., 1135$, 1137,1144

- als „Herr des Ermittlungsverfahrens“ 1020

- als Hilfsbeamte der Staatsanwaltschaft 598, 713, 722, 946, $1018 \mathrm{f} ., 1021 \mathrm{f} ., 1125$

- Belehrungspflicht bei Zeugenvernehmungen 1031

- Chef der Sicherheitspolizei und des SD s. auch Reichssicherheitshauptamt $184 \mathrm{f}$., $485,490,509,611,618,620,628$ ff., 676 , 690

- Einwirkung auf schwebende Verfahren 694 ff., 1119

- Emanzipation vom Reichsinnenministerium 553 ff., 559 f., 723, 1012, 1020, $1124 \mathrm{f}$.

- erhöhter strafrechtlicher Schutz für Polizeibeamte $912 \mathrm{f}$.

- Erzwingung der Vorführung $1031 \mathrm{ff}$.

- Exekutionen anstelle von Gerichtsverfahren 82, 597, 674-694, 899, 955, 1126

- Exekutionen zur Korrektur der Justiz s. auch „Erschießung auf der Flucht" 674, 679 ff., 683 ff., 694, 706, 847, 899, $911 \mathrm{f}$., $921,923,1080,1109$ f., 1126

- Exekutionen zur Strafverfolgung „Fremdvölkischer" s. auch Zivilarbeiter, polnische 689-694

- Exekutionszahlen $682 \mathrm{ff} ., 688 \mathrm{f}$.

- Kameradschaftsbund Deutscher Polizeibeamter 722,724 
- Mißhandlungen s. „Verschärfte Vernehmung" u. unter SS, Mißhandlungen

- Mitwirkung bei Begnadigungen $627 \mathrm{ff}$.

- Mitwirkung bei der Anordnung der Sicherungsverwahrung $729 \mathrm{f} ., 742,744 \mathrm{f} ., 1029$

- Reichskriminalpolizeiamt s. dort

- Reichssicherheitshauptamt s. dort

- Strafverfolgung durch die 196f., 199, $544 \mathrm{f}$., $547 \mathrm{ff}$., $554 \mathrm{ff}$., $560 \mathrm{ff}$., $584 \mathrm{ff}$., $597 \mathrm{ff}$., $603 \mathrm{f}$., $606 \mathrm{f} ., 609,621,689$ ff., 719 ff., 727,730 , $733,738,740,886,905,1001,1019,1021$, $1024,1026,1028,1044,1065,1089,1125 \mathrm{f}$, 1137

- Verhinderung von Ermittlungen 324, 329, 337 f., 350,382 f., 387 ff., 392, 394, 405 ff., 439f., 452, 474, 478, 488, $491 \mathrm{f}$., 584, 587, $592 \mathrm{ff} ., 597 \mathrm{f} ., 633,639,643 \mathrm{ff} ., 653 \mathrm{f} ., 676 \mathrm{f}$., 707, $712 \mathrm{f} ., 905,998,1022 \mathrm{f} ., 1119,1125 \mathrm{ff}$., 1127

- Verklammerung mit der SS 559f., 633, 1124

- „Verschärfte Vernehmung“ s. dort

- Zuständigkeitsabgrenzung zur Justiz 1018 ff., 1025 ff., 1089, 1125, 1127, 1145

Polizeiaufsicht $546,721,724,726,729 \mathrm{f}$., 742 , 778

Polizeihaft s. auch Schutzhaft und Vorbeugungshaft $67,340,545,549,597,604,690$, $700,823,1019$ f., 1023 f., 1044, 1066, 1125 , 1135

Polizeijustiz s. auch Polizei, Strafverfolgung durch die 322, $547 \mathrm{ff}$., $584 \mathrm{ff}$., 589, $597 \mathrm{ff}$., 621,675 ff., 685,689 ff., $956,1026,1126$ ff., 1132,1137

Polizeirecht $536 \mathrm{ff} ., 541 \mathrm{ff} ., 551,554,562 \mathrm{f}$., $597 \mathrm{f} ., 738,1021,1027,1031 \mathrm{f}$.

Polizeiverwaltungsgesetz, preußisches v. 1.6.1931 537f., 541 f., 545, 554

Pommern 769

Posen 287, 742, 744

Potempa-Fall 61, 65, 225, 326, 328, 828, 946

Potsdam $443 \mathrm{f}$.

Präsidialkabinett $57 \mathrm{ff}$., $62 \mathrm{ff} ., 1113,1115$

Präsidialkanzlei 209, 362, 372, 374, 605, 687 f., 708 f., 826, 829, 846, 923, 959, 1071

Prăsidialverfassung s. unter Gerichte

Prag 279, 742

Pranger $337 \mathrm{ff} ., 383,777$

Presse 25, 27, 53 f., 678 f., 683 ff., 755 ff., 773 , 799, 807, 822, 838, 873, 881, 895f., 903, $925,1008,1054$ f., 1059, 1111, 1123, 1126, 1142

- Angriffe auf die Justiz $663 \mathrm{ff} ., 669 \mathrm{f}$.

- -dezernenten bei den Gerichten 663

- -referent des Reichsjustizministeriums 663 , 666,670 f., 823,895
Preußen 5, 59f., $98 \mathrm{f} ., 101,103,107,112,120$, $127,129,135,140 \mathrm{f} ., 145,147,149 \mathrm{f} ., 154$, $158,161,163 \mathrm{f} ., 167,196,214,221 \mathrm{ff}$., 231 , 238, 240, 245, 301, 305, 314 ff., 320, 325 f., 328 ff., 334, 336-345, 345 ff., 424, 431, 478, 537, 542, $548 \mathrm{f}$., 553, $555 \mathrm{f}$., 574, 581, 700, $719 \mathrm{ff} ., 835,874,971,1096$

- Finanzminister(ium) 75, 132, $160 \mathrm{f.,} 200$, 826, 1011

- Kultusministerium 271, 284

- Landeskriminalpolizeiamt $724 \mathrm{f}$.

- Wirtschaftsministerium 554

Preußenhaus 962, 964

„Preußenschlag“ 49, 59, 132, 222, 323

Preußisch Eylau (Ostpreußen) 483

Preußisch-Süddeutsche Staatslotterie 695

Prittlbach (bei Dachau) 440

Privatklage $985 \mathrm{f} ., 992,1054 \mathrm{f} ., 1090,1096$

Privatrecht 927, 1132

Protektorat Böhmen und Mähren 279f., 291, 529, 744, 953

Prüfungswesen s. unter Justizbeamte

Prügelstrafe $646 \mathrm{ff} ., 710,716,718,776$

Ragnit (Ostpreußen) 462

Räterepublik München 16, $18 \mathrm{ff} ., 26,28$

Rassengesetzgebung $141,191,310,761 \mathrm{f}$., 770 f., 864 ff., 872 ff., 1102, 1130

"Rassenschande“ 185, 200, 492, 622f., 683, 698, 761 f., 770 f., 778, 801 f., 804, 862, 864 ff., 870 f., 874 ff., 884,1097 f., 1101 f., $1106,1123,1130,1141$

- Bestrafung der Frau 797, 881 ff., 1130

- Rechtsprechung $879 \mathrm{ff} ., 1101 \mathrm{f}$.

- Todesurteil 909

- Zahl der Verurteilten 879, 885

Rastatt (Baden) 301

Rauschtat 774, 784, 789, 799, 841 ff., 924

Rechnungshof des Deutschen Reiches 984

Rechtsangleichung

- im Saargebiet 118

- im Sudetenland 811

- in Österreich 260f., 811, 1085

Rechtsanwälte $70,97,101,113,163,229,266$, 268, 278 f., 282, 284, 289 f., 298 ff., 302,317 , 482 f., 546, 565, 572 f., 694 ff., 738 ff., 754, $769,785,965,974,1002,1006,1014,1045$, 1093,1114

- Altersversorgung 176

- „Dislozierung“ jüdischer $147 \mathrm{f}$.

- ehrengerichtliche Verfahren 698

- jüdische s. auch „Altanwälte“, jüdische und Armenanwälte, jüdische $70 \mathrm{ff}$., $89,124 \mathrm{ff}$., 139 ff., 158, 174 ff., 178 ff., 183, 322 f., 460 f., $635,661,1117$

- simultan zugelassene 174 
- Versorgung der jüdischen 175 ff., 180, 1117

- Vertrauensmänner der jüdischen Rechtsanwälte Deutschlands 184

- Vertretung von Schutzhäftlingen 546, 551f., 555, 564-573, 698, 1006, 1125

- weibliche $318 \mathrm{f}$.

- wirtschaftliche Lage $125,174,188,1114$

- Zahl der 119, 125, 149, 151, 174f., 237

- Zahl der jüdischen 136, 142, $146 \mathrm{ff} ., 149 \mathrm{ff}$., $174 \mathrm{ff}$.

- Zahl der „Mischlinge“ und „jüdisch versippten" $188 \mathrm{f}$.

Rechtsanwaltschaft 94, 121, 124, 175 ff., 206, 572,697

- Zulassung zur $125,127,132,135,139 \mathrm{f}$., 178, $181 \mathrm{f} ., 189,319,702 \mathrm{f}$.

Rechtsanwaltskammer s. Anwaltskammer

Rechtsanwaltsordnung 174, 189, 702

Rechtsbehelfe, außerordentliche 659, 944, 1011,1037 ff., 1082, 1085, 1087, $1109 \mathrm{f}$., 1126,1142

Rechtsbeugung 1035

Rechtseinheit 732, 834f., 850, 859, 862, 874, 954, 959, 967, 971, 983, 990f., 998, 1006, 1011, 1013f., 1037, 1061, 1082, 1084, 1086, $1098,1105,1136 \mathrm{f}$.

- Beauftragter des Führers zur Wahrung 998, 1006, 1008, 1010

Rechtserkenntnisquelle(n) 850f., 860, $872 \mathrm{f}$., 1130, 1139

Rechtskontinuität 1113

Rechtskraft der Urteile 839, 841, 884, 914, 948, $990 \mathrm{f} ., 993,1006,1009,1012,1037 \mathrm{f}$., $1040,1060,1063,1066 \mathrm{f} ., 1072,1074 \mathrm{f}$, $1082 \mathrm{ff} ., 1088,1109,1132,1135 \mathrm{f} ., 1141 \mathrm{f}$.

Rechtsmittel s. auch Berufung, „Rechtsrüge“ und Revision 26, 43, 540, 542, 544, 554, $556,569,668,729,932,938 \mathrm{ff} ., 942 \mathrm{ff} ., 948$, 954, 960, 976, 982, 987, 989 ff., 997 f., 1002, $1004,1006 \mathrm{ff} ., 1011,1014,1032,1037 \mathrm{f}$., 1044, $1058 \mathrm{ff} ., 1063 \mathrm{f} ., 1066,1069,1071$, 1079, 1082, 1084, 1096, 1109, 1125, 1134, $1136 \mathrm{f} ., 1142$

- Ersetzung durch den Spruch einer politischen Instanz $997 \mathrm{f}$., $1007 \mathrm{ff} ., 1038$

Rechtspositivismus 1, 848 f., 860, 872 f., 978

"Rechtsrüge“ („Urteilsrüge") s. auch Revision 932 f., 936, 938, 989 ff., 1012 f., 1036 f., 1135

- Prüfung tatsächlicher Feststellungen 990, 997 f., 1014, 1036

Rechtssicherheit 67, 163,324, 352, 372, 450, 462, 475, $494 \mathrm{f}$., 510, $514 \mathrm{f}$., 545, 552, 556, 568 f., $617,680,691,694,722,752,813$, $849,862,874,896,909,919,921,1012$, $1028,1088,1108,1118,1120,1125$ f., 1130 , $1137,1140,1145$
Der Rechtsspiegel 671

Rechtsstaat 67, 69, 76, 78, 122, 451, 495, 557, $567 \mathrm{f} ., 826,830,862,926,1115,1129,1146$

Rechtsstaatlichkeit $69,78,83,122,133,289$, 333, 451, 475, 711, 790, 828 ff., 897, 909, $925,952,973,992,1009$ f., 1026, 1043, $1091,1111,1114,1125,1128,1135,1143 \mathrm{ff}$.

Rechtsstudenten 312 ff., 1043

- Zahl der $312 \mathrm{ff}$.

Rechtsvergleichender Kongreß v. August 1937 im Haag 863

Rechtswidrigkeit, fehlendes Bewußtsein der 19, 467 f., 486, 490, 712, 774, 797, 813

Rechtswissenschaft $746,754,765,827 \mathrm{f} ., 927$, 980, 984, 1003, 1006, 1015, 1017, 1132

- „Kieler Schule“ 765

Referendare 87, $109 \mathrm{ff} ., 137,153,166,202$, 216, 234, 267, 293, $299 \mathrm{ff} ., 312 \mathrm{ff} ., 318,979$

- Arbeitsgemeinschaften 301, 304, $308 \mathrm{ff}$.

- Besoldung 314

- jüdische 128, 140, 153f., 166, 634

- wirtschaftliche Lage 314

- Zahl der 314

Referendarinnen 319

Referendarlager s. Gemeinschaftslager „Hanns Kerrl" und "Hans-Frank-Lager"

reformatio in peius 982, 991, 1050, 1058, 1063, 1135f.

Regensburg $10 \mathrm{ff},{ }^{16}$

Regierung

- bayerische 18, $20 \mathrm{ff} ., 24 \mathrm{ff}$., $34,37 \mathrm{ff} ., 41 \mathrm{f}$., 47 f., $51 \mathrm{ff} ., 56,75,85,115,129,162,230 \mathrm{f}$., 323,332 ff., 388 ff., 395 ff., $404,406,410$ f., $424,433,548,551,586,637$ ff., 642, 834, 838,1011

- niederländische 834

- preußische 59f., 85, 120,132, 140,160, 222, 229, $326 \mathrm{f}$., $330,553 \mathrm{ff}$., 568, $570 \mathrm{f}$., 646, $700,831,886,1011$

- sächsische 537

„Regierungsgesetze“ $68,1115,1144$

Rehling (Oberbayern) 399

Reichenhall (Oberbayern) 413

"Reichsärzteführer“ s. auch "Reichsgesundheitsführer" 499f., 874

Reichsanwalt(schaft)

- beim Reichsgericht 84, 138, 167, 171, 217 , $248,258,263,270,294,418,949,970,993$, 1085, 1099

- beim Volksgerichtshof 208, 217, 229, 263, 270, 283 f., 294, 594, 618, 645, 713, 960, 965, 968 ff., 993, 1100

- beim Volksgerichtshof, Zahl der Mitarbeiter 968, 971

Reichsarbeitsdienst (RAD) s. auch Arbeitsdienst 308 f., 801, 819, 916 
Reichsarbeitsgemeinschaft Heil- und Pflegeanstalten 503

Reichsarbeitsminister(ium) 94, 199, 689, 833, 937, 1012

Reichsausschuß zur wissenschaftlichen Erfassung von erb- und anlagebedingten schweren Leiden 501

Reichsaußenminister s. Auswärtiges Amt

Reichsbanner 59, 127, 138, 230, 249, 661

Reichsbeamtengesetz vom 31.3.1873 137, $189 \mathrm{f}$.

Reichsbesoldungsordnung 118, 293

Reichsbürgergesetz 165, 173 ff., 177, 242, $251 \mathrm{f}$.

- zahlenmäßige Auswirkung seiner Durchführung bei der Justizbeamtenschaft $170 \mathrm{f}$.

Reichsbund der Deutschen Beamten (RDB) 87 f., 217, 251, 256, 797

- Fachschaften 157, 246, 256

Reichsbund der Kinderreichen (RdK) 251

Reichsdienststrafordnung (RDStO) v. 26.1.1937 193

Reichserbhofgericht 996

Reichsfinanzminister 59, 94, 111, $116 \mathrm{f} ., 137$, 139, 164, 177, $257 \mathrm{f} ., 273,308,314,317$, $734 \mathrm{ff} ., 750,835,888,937 \mathrm{f} ., 967,1011,1052$

Reichsfinanzministerium $187,190,199$, 238 f., 256, 262, 264, 607, 654, 1011

Reichsfluchtsteuer 1066

Reichsfrauenführung 319

Reichsführer-SS und Chef der Deutschen Polizei s. auch im Personenregister unter Himmler 184f., 380, 412, 424, 440, 465, 484, 521, 551 f., 559, 561, 571, 573, $590 \mathrm{ff}$., 596 f., $602,607,618,621$ f., $626,633,651$, 657 f., $668,673,677$ f., $682,684,690,692$, 701,724 ff., $740,796,801,884,911,1020 \mathrm{f}$., 1026,1124

Reichsgericht $2,14,28,79,84,86,95,119$, 121,124 ff., 131, 138, 165,167, 171, 208, $217,242,248,253,257 \mathrm{ff}$., 261, 263, 270, 273, 277, 290f., 294, 334, 417 ff., 424, 468, $527,540,543,548,604,664 \mathrm{f} ., 671,705$, $714,728,732,763,769$ f., 804,823 f., 826 ff., $830,837,845,848$ ff., 852 f., 859,861 f., 869 , 882, 890, 894, 899, 932 f., 936 ff., 940,943 , $947,949,953,956$ ff., 966 ff., 973 ff., 978 ff., 983, 989 ff., 993, 1011 ff., 1017, 1019, 1023, $1033,1037,1039 \mathrm{ff} ., 1051 \mathrm{f} ., 1061,1063$, $1071,1074,1078,1081,1083$ ff., 1087, $1098,1100,1108$ f., 1129 f., $1132 \mathrm{ff} ., 1136 \mathrm{ff}$.

- Analogieanwendung 861

- Besonderer Strafsenat 680, 684, 913, 943, $979,1011,1035,1040$ ff., 1072 ff., 1085 , $1089,1136 \mathrm{f}$.

- Fernsprechautomatenurteil v. 18.12.1933 788,848 f., 853,1130
- Großer Senat für Strafsachen 862, $879 f$., 933, 936, 972, 1063

- Großer Senat für Zivilsachen 933, 936, 972, 1063

- „Oberster Senat“ $1038 \mathrm{f}$.

- Plenum 971 f., 1063

- Rechtsprechung $1062 \mathrm{f} ., 1071$

- Rechtsprechung zum Blutschutzgesetz $879 \mathrm{ff}$.

- vereinigte Große Senate 933, 972

- vereinigte Senate $971 \mathrm{f}$., 1063

- Zivilsenate 971

„Reichsgesundheitsführer“ s. auch „Reichsärzteführer" 503, 523

Reichsgrundsätze über Einstellung, Anstellung und Beförderung der Reichs- und Landesbeamten v. 14. 10.1936 255f., 259 f., 262

Reichsinnenminister 58f., 67, 76, 83, $92 \mathrm{f}$, $105,117,138$ f., 162, 186, 201, 206, 214, 228, 238, 257 f., 273, 277, 329, 368, 381, $406,425,441,456,479,501,565 \mathrm{f} ., 647$, 733 f., 796, 823, 826, 833, 888, 903, 937, $963,967,984,1055$

Reichsinnenministerium $60 \mathrm{f} ., 77,129,132 \mathrm{f}$., 143,157 f., 165,168 f., $172,177,181,185$, 187, 189 f., 194, 197 ff., $215,255,259,262$, $271,292,417,423 \mathrm{f} ., 426,430,456,463$, $480 \mathrm{ff} ., 500 \mathrm{ff} ., 506,513,516,522,525$, $541 \mathrm{f}$., $546 \mathrm{ff}$., $550 \mathrm{ff}$., $559,567 \mathrm{f}$., $570,575 \mathrm{f}$., $607 \mathrm{f} ., 630,632,650,654,694 \mathrm{f} ., 699 \mathrm{f}$., 704 ff., 709, 723, 740 ff., 772, 801, 821, 834, 866,870 f., 873 ff., 877 f., 882 , 884 ff., 906 , 961 f., 999, 1012, 1017, 1020,1120,1125, 1127, 1130

„Reichsjuristenführer“ s. auch „Reichsrechtsführer" $176,568,869$

Reichsjustizamt 84, 86, $241 \mathrm{f}$.

"Reichsjustizkommissar" s. Reichskommissar für die Gleichschaltung der Justiz in den Ländern und für die Erneuerung der Rechtsordnung

Reichsjustizministerium

- Abteilung I 106, 175, 242, 244, 247, 260, $446,573,701,994,1014$

- Abteilung II 242, 244, 247, 260f., 367, 416, 554, 655, $735 \mathrm{f} ., 739,743,754,771,792 \mathrm{f}$, 805 ff., 818, 834, 851 ff., $861,870,882$ f., 886f., 916 f., 919, 924, 931, 935, 941, 981, 994, 1015

- Abteilung III 214, 244, 246f., 260, 264, 270,346 f., $359,518,523,540,561,570$, $578,591,601,608,611,618,620,626$, 652f., 681, 700, 713, 718, $727 \mathrm{f}$., 732, $735 \mathrm{f}$., $739,743,774,879,882$ ff., $888,916,918 \mathrm{f}$, 935, 954, 981, 994, $1013 \mathrm{ff} ., 1022,1077$, 1095, $1099 \mathrm{f}$. 
- Abteilung IV 106, 174, 187, 244, 258, 260 265, 771 f., 870, 927, 972, 994, 1014

- Abteilung V 244, 247, 258, 260, 262, 264, $541,554,570,591,608,701,994,1014$

- Abteilung VI 244, 258, 260, 994

- Abteilung VII 219

- Abteilung VIII 260

- Abteilung XV 233

- Abteilung Bayern 112

- Abteilung LA 244, 247, 258

- Abteilung Nord 112

- Abteilung Österreich 174, 260f., 278, 286, 581 f., 596

- Abteilung RJP 244

- Abteilung Sachsen-Thüringen 110, 112

- Abteilung Württemberg-Baden 110, 112

- Abteilungen 86, 106, $110 \mathrm{ff} ., 119,198,231$, 235 f., 243 f., 247, 250, 274

- Beauftragte $110 \mathrm{ff} ., 581$

- Beteiligungsreferenten 1095

- Bezirksreferenten 1095, 1105, 1107

- „Braunes Buch“ 218

- Diensttagebuch 254, 873

- Frage des „Versagens“ $1143 \mathrm{ff}$.

- "Geheime Kanzlei“ 1094

- Gesetzgebungstätigkeit 5 f., $49 \mathrm{f}$., $65,84,86$, 89 f., 95,100 f., $106,120,244,687,740$ f., 746 ff., 751 ff., 758 ff., 792 f., 819 ff., 835 f., 873 f., 882 ff., 886 ff., 901 ff., 915 ff., 919 ff., 924 ff., 931 ff., 944 ff., 1049 ff., 1068 ff., $1090 \mathrm{ff} ., 1110,1115,1128 \mathrm{ff} ., 1144$

- Ministerbüro 106, 360

- Ministerialdirektoren 219, 241 ff., 248, 251 f., 258, 260, 262 ff., 284, 325, 359, 416, $431,446,518,523,527,540 \mathrm{f} ., 570,573$, 578, 594f., 601, 611, 626, 713, 715, 727, $754,771,807,834,851,858,882,916 \mathrm{f}$., $919,927,931,964,972,981,983,1015$, 1023 , 1032, 1038, 1077, 1094f., $1099 \mathrm{f}$., $1103,1105,1107$

- Parteigenossen im 218f., $1117 \mathrm{f}$.

- Pressereferent s. unter Presse

- Sachreferenten 1095, 1105

- Sonderreferat für Sondergerichtssachen $954 \mathrm{f} ., 1104$

- Sonderreferenten 1095

- Staatssekretär s. dort

- Unterstaatssekretär 260, 268, 807

- Zahl der Ministerialbeamten 86, 106, 219, 243, 245 ff., 260, 264, 266

- Zentralabteilungen 106, 244

- Zentralstaatsanwaltschaft s. dort

Reichs-Justizprüfungsamt 106, 109 ff., 219, 224, 247, 267, 270, 301, 303, 1003

Reichskabinett s. Präsidialkabinett und Reichsregierung
Reichskanzlei $34,73,83,86,96,100,103$, $111,116,178 f ., 187,199,203,243,249$, $257,259,277,447,500,506,522 f$., 546, $665,679,687,747,750,752,759,792,794$, $796,802,806,811,818,821,823,826,846$, 865, 891, 895, 898, 903, 906, $910 \mathrm{f}$., 922, 924, 962 ff., 967 f., 993, 1038, 1046, 1049, 1119

- Chef der 94, 103, 126, 135, 178f., 187, 193 ff., 256 f., 275 f., 421, 429, 431, 498, 507, $520,533,597,737,747$ ff., $756,773,793$ ff., 799 f., 802, 805, 807, 811, 818, 824, 829, 890, 895f., 898, 902, 959, 962, 966, 968, 973, 1046 ff., 1072 f., 1076

Reichskirchenminister(ium) s. Reichsminister(ium) für die kirchlichen Angelegenheiten

Reichskolonialbund 251

Reichskommissar

- für den freiwilligen Arbeitsdienst 304

- für die Behandlung feindlichen Vermögens 247

- für die besetzten niederländischen Gebiete 246

- für die Gleichschaltung der Justiz in den Ländern und für die Erneuerung der Rechtsordnung („Reichsjustizkommissar") 86, 88 ff., 93, 95, 102, 104, 110, 115, 144 ff., 234 f., 428, 546, 747, 754 f., 834, 933, 981, 1116

- für die sudetendeutschen Gebiete 278

- für die Wiedervereinigung Österreichs mit dem Deutschen Reich 178, 260, 286

Reichskommissariat

- für die besetzten rheinischen Gebiete 265

- Ukraine 284

Reichskonkordat 1030

Reichskriegsgericht 259, 901, 960, 1071, $1073 \mathrm{f}$.

- Sondersenat 1072, $1075 \mathrm{f}$.

Reichskriegsminister(ium) 796, 800 ff., 886 ff., 937, 965, 1012, 1015

Reichskriminalpolizeiamt $683,724,726 \mathrm{f}$., $729 f ., 733,738,744$

„Reichskristallnacht“ 5, 183, $484 \mathrm{ff} ., 534,809$, $815,825,1048,1102,1122 \mathrm{f}$.

Reichsluftschutzbund 219, 251, 806, 833

Reichsminister der Luftfahrt 806, 833, $886 \mathrm{f}$., 961, 963, 970

Reichsminister(ium) des Innern s. Reichsinnenminister(ium)

Reichsminister(ium) für die kirchlichen Angelegenheiten 103, 521, 689, 794, 807, 891, $938,1011,1030$

Reichsminister(ium) für Ernährung und Landwirtschaft 199, 243, 890, 1011 
Reichsminister(ium) für Volksaufklärung und Propaganda s. Reichspropagandaministerium

Reichsminister(ium) für Wissenschaft, Erziehung und Volksbildung 172, 246, 300, 787

Reichsnährstand 890

Reichsnotarkammer 88, 108

Reichsnotarordnung 88, 795

Reichspatentamt 84, 95, 119, 124, 138f., 165, $167,171,187,208,243,270,291,527,974$

Reichspostminister(ium) 80, $848 \mathrm{f} ., 853,1011$, 1059

Reichspräsident 9f., 24, 32 f., 57, 62, 66, 68, 89, 93 f., 134, 325, 334 f., 393, 451, 454, 535, $644,755,823,826$ ff., 844, 846, 960, 1113, 1128

- Büro des s. Präsidialkanzlei

- Notverordnungsrecht 57,68,325, $826 \mathrm{ff}$.

Reichspropagandaministerium 77, 499, 513, $521,664,671,772,902,904,1011$

Reichs-Rechtsanwaltskammer $88,150,174$, $176,180,183,185,189,567 \mathrm{ff} ., 571 \mathrm{ff}$., $695 \mathrm{f} ., 1015$

Reichs-Rechtsanwaltsordnung 88, 319

„Reichsrechtsführer“ s. auch „Reichsjuristenführer" 64, 567, 569, 747, 994, 1008

Reichsregierung s. auch Präsidialkabinett $9 \mathrm{f}$., $23 \mathrm{f}$., 31 f., $34 \mathrm{f}$., $48 \mathrm{ff}$., 55, 57 ff., $63 \mathrm{ff} ., 67,85$, $88 \mathrm{f} ., 94,110 \mathrm{f}$., 116,131 f., $134 \mathrm{ff} ., 140,142$, 146 ff., 158 f., 162, 164, 204, 219, 326 f., 329, 335, 414, 418, 425, 441, 447 ff., 465, 480f., 496, 507, 535 ff., 539, 559, 621, 664, 719, $740,748 \mathrm{ff} ., 753,756,760,768,773$, 792-804, 811 f., 816, 818, 820 f., 823 ff., 831, 844 ff., 858, 866, 869, 874, $891 \mathrm{f}$., 897, 919 , 944 ff., 957 ff., 963,966 f., 981, 984, 1045, 1050,1052 , 1055, $1072 \mathrm{f} ., 1092,1113 \mathrm{f}$., 1119,1129

- Geschäftsordnungen v. 3.5 .1924 und 20.3.1935 829

- Kritik an der Justiz $679,946,1126,1138$, 1141

- Zahl der Sitzungen 749

Reichs-Rundfunk GmbH 664

Reichssicherheitshauptamt s. auch Polizei, Chef der Sicherheitspolizei und des SD 270, 536f., 591, 597, 611f., 631, 685, 690, 692, 702, 730, 738, 742

Reichsstatthalter 96, 99, 112 f., 329, 334, 369, $548 \mathrm{ff} ., 561,642,644,835,846,869,1055$, 1115

- in Bayern 230f., $233 \mathrm{ff}$., 323, $332 \mathrm{ff}$., 380 , 382 f., 386 ff., 392 f., 408, 428, 435 f., 441, $550 \mathrm{f} ., 638,640,644,722,834,964$

- in Hamburg 374, $378 \mathrm{f} ., 669,705$

- in Hessen-Nassau 245, $271 \mathrm{f}$.
- in Oldenburg und Bremen 362

- in Posen 280

- in Preußen 221

- in Sachsen 368

- in Thüringen 109

- in Wien 173, 178

Reichsstelle

- für Raumordnung 103

- für Sippenforschung 170, 172, 252

Reichstag 9f., 22, $26 \mathrm{f}$., 52, $57 \mathrm{ff}$., $62 \mathrm{f} ., 65,68$, $85,99,130,133,168 f ., 195,226,267,280$, $321,376 \mathrm{f}$., 434, $457 \mathrm{f.,} \mathrm{477,} \mathrm{545,} \mathrm{725,} 749 \mathrm{f}$., 753,760 f., $795,799,808,820$ f., 826, 828 ff., 864, 875 f., 919, 957 ff., 1059, 1128 ff., 1141

- Wahl v. 5.3.1933 322

- Wahl v. $12.11 .1933348,755,757$

- Wahl v. 10.4.1938 805

Reichstagsbrand 824f., 1114

Reichstagsbrandprozeß 830, $956 \mathrm{ff}$., 1132

Reichstagsbrandverordnung (Verordnung des Reichspräsidenten zum Schutz von Volk und Staat vom 28.2.1933) $66 \mathrm{f}$., $321 \mathrm{f}$., $535 \mathrm{f}$., $548 \mathrm{f}$., $719,725 \mathrm{f} ., 823,827,830 \mathrm{f}$., 844 ff., 897, 900, 947, 954, 957, 960, 1100, $1114,1124,1144$

Reichs- und Preußisches Justizministerium 105f., 109, 112, 120, 244 f., 588, 604, 1091, 1116

Reichsverkehrsminister(ium) 80, 442

Reichswehr s. auch Wehrmacht 18, $29 \mathrm{f}$., $32 \mathrm{f}$., $35,39,62,66,75,121,303,305,392,414$, $417,419,433,450,463,466,963$

Reichswehrminister(ium) 32, 58, 60, 62, 68, 75, 94, $158 \mathrm{f}$., 325, 414, $417 \mathrm{ff} ., 463,834$, 838, 855, 944 f., 961

Reichswirtschaftsminister(ium) 72 f., 94, 199, 484, 772, 796, 802, 818, 834, 858, 873

Religion 782f., 810, 815, 819

Religionsgesellschaft(en) $\mathbf{7 8 2 \mathrm { f } . ,} 809,815 \mathrm{f}$., 1055

Remmert-Prozeß 365 f.

Remscheid (Rheinprovinz) 704

Repetitoren, jüdische 300

Republikanischer Richterbund 138, 224, 230 , 249, 1143

Republikschutzgesetz 23, 32, 34, 36, 44, 761, 864

Ressortegoismus 78, 227, 428, 510, 752, 1009, 1043, $1144 \mathrm{f}$.

Revision s. auch „Rechtsrüge“ 405, 540, 665 , $728,732,778,780,859,861 \mathrm{f} ., 882,899$, 932, 940, 944 f., $948,952,958$ f., 961,967 , 976 f., 982 f., 991,997 f., 1006, 1008, 1010 f., 1014, 1016, 1037, 1058f., 1061, 1063, 1068, $1071,1081,1083,1089,1098,1100,1108 \mathrm{f}$., $1134 \mathrm{ff}$. 
Revolution

- bolschewistische 1113

- nationalsozialistische 67, 122, $129 \mathrm{f} ., 141 \mathrm{f}$, $145,231,259,320 \mathrm{ff}$., $328 \mathrm{f} ., 331,336,339 \mathrm{f}$., $342,362,385,388,395,397,400,412,433$, $476,487,546$ f., 664, 703, 759, 832, 1119 , 1121

- von 191816 ff., 26, 66, 231, 255, 956

Rheinpfalz 301, 324

Rheinprovinz 539

Rheinsberg (Mark Brandenburg) 772

Richter s. auch Justizbeamte $26,37,101,121$, 137, 202, 214f., 218, 220, 263, $266 \mathrm{ff}$., 289 ff., 299,301 f., 304, 309, 313, 316, 419, $422,680,785,832,863,888,899,940,949$, $1102 \mathrm{f}$.

- Ablehnung wegen Befangenheit 1051

- Abordnung der 975

- Anpassung der $1142 \mathrm{f}$.

- Berufsrichter 932ff., 936, $938 \mathrm{ff} ., 947,959$, 961, 967, 976, 983, 1132

- „des Führers“ 1048

- Ehrenschutz gegenüber Angriffen 664, $666 \mathrm{ff}$., 672, 674, 1009

- Ermittlungsrichter 960, 1051

- gesetzlicher $37,935,975,978,1133 \mathrm{f}$.

- Hilfsrichter 317, 954, 968 ff., 975, 1064, 1135

- „jüdisch versippte“ 972

- jüdische $124 \mathrm{ff} ., 140 \mathrm{ff} ., 157,160,162,166 \mathrm{f}$., 169 ff., 230, 237, $322 \mathrm{f}$., 650,1117

- Leitsätze über Stellung und Aufgaben v. 14.1.1936 1139

- „Oberster“ s. dort

- weibliche $318 \mathrm{f}$.

- Wirkung der polizeilichen Urteilskorrektur auf die 599 ff., 625 f., 921, $1027 \mathrm{f.,} 1142$

- Zahl der $298 \mathrm{f}$., $317 \mathrm{f}$.

- Zahl der jüdischen 160

- Zwangsversetzung 129f., 143, 160, 162, 164 f., 173,195 ff., 671 f., 973 ff., 1119,1133 , 1142

Richteramt, Befähigung zum 94, 292, 296, $302,960,968,975,1007,1038,1075,1133$

„Richterbriefe“ s. unter Lenkung der Rechtsprechung

Richtergesetz 290

Richterrecht 132, 317, 419, 975

„Richtlinien für das Strafverfahren“ 496, 588 ff., 732, 893, 1023, 1065 f., 1096

Roding (Oberpfalz) $381 \mathrm{f}$.

„Röhm-Aktion “ 5, 227, 335, 352, 364, 393, 396, 399, 405 f., 410, 429 f., 433 ff., 463, 467, 469,471 ff., 476 ff., 484, 494 f., 534, 549 f., $552 \mathrm{f} ., 675,748,825,963,1120 \mathrm{ff}$.

- Zahl der Opfer 436, 441, 449, 457 f., 464, 706
Rom 11, 805

Rostock (Mecklenburg) 114, 766

Rotfrontkämpferbund 705

Rückwirkung von Gesetzen $6,61,68,416$, 763 f., 773 f., $798,806,826$ ff., 830 f., 834 ff., $841,843,846,851,855,860,894$ ff., 899, $910 \mathrm{ff} ., 914 \mathrm{ff} ., 918 \mathrm{f} ., 925,957,959,1071$, $1076,1079,1110,1128,1131,1136,1140$, 1144

Ruhestand 117, 127, 161, 166f., 170, 219, 225, 228 f., 236 f., 240, 242

- einstweiliger 137, 223 f., 281

- zwangsweise Versetzung in den s. Zwangspensionierung

Rumänien 697

Rußland s. Sowjetunion

SA (Sturmabteilung) $5,33,43,61,65,67,136$, 153, 193, 209, 212, 214, 216, $218 \mathrm{ff} ., 235$, 250, 268 f., 274, 301, 303, 305, 309, $320 \mathrm{ff}$., $329,331,336 \mathrm{ff} ., 353 \mathrm{ff} ., 371,394,407 \mathrm{f}$., $412 \mathrm{ff}$., $433 \mathrm{ff}$., $440,442 \mathrm{ff}$., $449 \mathrm{f}$., $454 \mathrm{f}$., $457 \mathrm{ff}$., $474,477 \mathrm{f} ., 485 \mathrm{ff}$., 532, 549, 593, 642, 662, 675, 703, 784, $786 \mathrm{f}$., 797, 824, $828,831,871,949,951,963,969,1091$, $1099,1119 \mathrm{ff}$.

- Allgemeine Dienstordnung 425, 427, 493

- Ausschreitungen $124 \mathrm{ff}$., 274, $320 \mathrm{ff}$., $328 \mathrm{ff}$., 337 ff., 342, 345, 353 f., 358, 368 f., 372, 375, 380 ff., 386 ff., 403 ff., 412 f., 420, 472, 485 ff., 492, 965, $1119 \mathrm{ff}$.

- Brigade Schleswig 341

- Brigade Württemberg-Süd $\mathbf{4 2 0}$

- erhöhter strafrechtlicher Schutz $912 \mathrm{f}$.

- Feldjäger 350, 355, 364f., 424, 477

- Gruppe Berlin-Brandenburg 321, 343, 442

- Gruppe Franken 389, 391

- Gruppe Niederrhein 428

- Gruppe Nordsee 364

- Gruppe Nordmark 338 ff., 342

- Gruppe Sachsen 369

- Gruppen 424, 428

- Kommissare 336, $380 \mathrm{ff} ., 384 \mathrm{ff} ., 389,394$, 396 ff., 407, 547, 999

- Mord-Verfügung Röhms v. 31.7.33 329, $369 \mathrm{f}$.

- Oberste SA-Führung s. dort

- Stabschef 321, 329, 331, 343, 356, 359, 364, $369,372,380,382,389,396$ ff., 424 f., 431 , $434,437,472,478,532,748,961,963,1120$

- Strafgerichtsbarkeit 5, $338 \mathrm{f}$., $341 \mathrm{ff.}$., 390 , 400 ff., 412 ff., 433, 454, 477, 1120, 1127

- Zahl der zurückgestellten Strafverfahren 345,1120

Saarbrücken 118, 124, 317

Saarland 117 f., 283, 767, 875, 1116 
Saarlouis (Saarlautern) 118, 283

Sachenrecht, Gesetzgebung 928

Sachsen 32, $98 \mathrm{f}$., 107, 109, 112, 114, $238 \mathrm{f}$., 245 f., 259, 262, 372, 537, 589, 964

Salzburg 181, 805

"Der SA-Mann" 671

Schadenersatz 482 f., 544, 558, 984, 1090

Schaumburg-Lippe 112

„Schießerlaß“ Görings v. 17.2.1933 320f., 329

Schlesien 434, $455 \mathrm{ff}$.

Schleswig (Schleswig-Holstein) 337,340

Schleswig-Holstein 157

Schneidemühl (Pommern) 184

Schnellverfahren 982, 991, 1050, 1070, $1088 \mathrm{f} ., 1096,1135,1137$

Schöffen 38f., 371, 477, 832, $934 \mathrm{ff}$., 938, 942, 949, $975 \mathrm{f} ., 1009,1093,1133$

- Ernennung 934, 936 ff., 944 ff., 983

- jährlicher Bedarf $941 \mathrm{f}$.

- jüdische 128, 945

Schöffengericht(e) s. Amtsgericht(e)

Schuldrecht, Gesetzgebung 927

Schulung, weltanschauliche der Justizangehörigen $140 \mathrm{f}$., $300 \mathrm{ff}$., $307 \mathrm{ff}$., $312,668,1138 \mathrm{f}$

"Schulungshaft" für zurückgekehrte Emigranten $607 \mathrm{ff}$.

Schutzhaft $5,67,75,78,124,182 \mathrm{ff} ., 197,254$, 321 f., 324, 345, 349, 353 ff., 357 f., 368, 383, 394, 429, 451, 478, 537 f., 544-632, 653, 658, 676, 704, 709, 719, 726f., 730, $739 \mathrm{f}$., $883,905,1014,1021,1024,1028,1044$, 1065, 1124 ff., 1130

- Anrechnung auf die Strafe 594, 599 f., 603 bis 610

- gegen Beamte 555

- gegen begnadigte Strafgefangene $627 \mathrm{ff}$.

- gegen Prozeßbeteiligte 694 ff., 1119

- gegen Rechtsanwälte 483, 549, 566, 569, $698 \mathrm{ff}$.

- gegen Richter 1111

- gegen weibliche „Rasseschänder“ 883 ff.

- Geschichte der 545

- nach gerichtlichem Freispruch 563, 599-603, 740, 958, 1021, $1110 \mathrm{f} ., 1125 \mathrm{f}$.

- nach Verbüßung der Freiheitsstrafe 606, 612-626, $1125 \mathrm{f}$.

- nach Verbüßung der Freiheitsstrafe bei Bibelforschern 620, $623 \mathrm{f}$., 1126

- nach Verbüßung der Freiheitsstrafe bei Hochverrätern $613 \mathrm{ff} ., 623,778,1126$

- nach Verbüßung der Freiheitsstrafe bei Juden 623

- nach Verbüßung der Freiheitsstrafe bei Landesverrätern 612 ff., $623,778,1126$

- nach Verbüßung der Freiheitsstrafe bei Polen 623
- nach Verbüßung der Freiheitsstrafe bei Rasseschändern $622 \mathrm{f}$., 778, 879, 1126

- Unterbrechung zur Strafvollstreckung $606,610 \mathrm{ff}$.

- Zahlen 486, 573, 579, 581, 625, 658

- zur Korrektur der Justiz 550, 563, 583-632, $663,667,680,879,958,1028,1100,1110 \mathrm{f}$., $1125,1141 \mathrm{f}$.

Schutzhaftbestimmungen $547 \mathrm{ff}$., $560 \mathrm{ff}$., 564 , $575,591,700,702$

- des RMdI v. 12./26.4.1934 478, 538, 548 ff., 558, 560, 569, 576, 584, 586, 590 f., $603 \mathrm{f} ., 609,647,699,702,1125$

- des RMdI v. 25.1.1938 $561 \mathrm{f}$., 584, 591, $602,700,727,1125$

- Görings v. 11.3.1934 $548 \mathrm{f}$., 551, $564 \mathrm{f}$.

Schwabhausen (bei Dachau) 438

Schwangerschaftsunterbrechung s. auch Abtreibung $500 \mathrm{f} ., 801 \mathrm{f}$.

Schwarzburg (Thüringen) 797, 1008, 1017

„Das Schwarze Korps“ 572, 646, 651, 659, 663-675f., 682, 870 f., 1003

Schweiz 687, 826f., 923

Schwerin (Mecklenburg) 114

"Schwerverbrecherverordnung “ (Entwurf) 820, 912, 917 ff., 922

Schwurgericht(e) 932f., 936, 939, 941 f., 944, 953, 976f., 982 f., 989, 1051, 1062, 1064, $1069,1089,1134$

Separatismus 20,38, 62, 324

Sicherheitsdienst des Reichsführers SS (SD) $210,243,270,434,438,440,460,464,471$, $485,500,510,533,620,659,666,683,954$, $994,1003,1095$

Sicherungsverfahren gegen Schuldunfähige 992, 1025 ff., 1057 f., 1135

Sicherungsverwahrung $313,534,652,675$, $680,688,719 \mathrm{f} ., 723,727-733,735 \mathrm{ff}$., 740 ff., 777 f., $780,788,838,840$ f., 846, 894, 910 f., $917,924,936,942,977,979,1027$ ff., 1058, 1069, 1082, 1093, 1096, 1102, 1110, $1126,1128,1134$

- Auslieferung Sicherungsverwahrter an die Polizei 738,745

- Zahlen 723, 727 f., 731f., 736, 745, 844

Soest (Westfalen) 517

Sofia 830

"Sonderbehandlung“" s. auch Polizei, Exekutionen 597, 647, $676 \mathrm{f}$., 682,690

Sondergericht 543 f., 677, 942, 978 f., 1039 , 1083 f., 1086, 1091, 1134, 1141

- Altona 541, 661

- Bamberg 948

- Berlin 77, 348, 599, 679, 683 f., 899, 911

- Beuthen 61,326,946

- Breslau 541, 683, 947, 1081 
- Darmstadt 543

- Dortmund 953

- Dresden 948

- Düsseldorf 521, 953, 1104

- Essen 948

- Feldkirch (Vorarlberg) 1104

- Frankenthal (Pfalz) 947

- Frankfurt a. M. 599

- Freiberg (Sachsen) 589, 947

- Halle 947

- Hamburg 540, 600, 623, 899, 948, 953

- Hannover 948, 1111

- Kattowitz 688

- Koblenz 948, 954

- Köln 594, 948

- Leipzig 948

- Linz 1087

- Luxemburg 948

- Mannheim 899

- München 477, 681, 692, 825, 961

- Nürnberg-Fürth 899, 909

- Reichenberg (Sudetenland) 279

- Salzburg 1104

- Stuttgart 510, 522

Sondergerichte $6,185,220,327,346,416 \mathrm{f}$, 420 f., 423, 428, 496, 550, 619, 678f., 900, 904, 907, 913, 915, $931 \mathrm{f.,} \mathrm{944,} \mathrm{946-956,}$ 959, 961f., 970, 973, 976f., 1057, 1062, $1074,1082,1086$ f., 1095 f., 1102 ff., 1107 , 1126, 1132, 1137, 1141, 1143

- Berufung der Richter 940, 947 f., 954

- Besprechung der Vorsitzenden im Reichsjustizministerium v. 24.10.1939 901, 916, 950, 956, 1069, 1094, 1101 ff., 1106

- Errichtung 946ff., 953, 955

- in der Weimarer Republik 946

- Kritik des Reichsjustizministeriums an Urteilen $1104 \mathrm{f}$.

- Verfahrensvorschriften 550, 678, 906, 940, $948 \mathrm{f} ., 951,954,988,1000,1050,1052$, $1064,1066,1069,1076,1082,1126,1132$, 1136

- Zahl der 947, 953

- Zahl der Urteile 953

- Zuständigkeit 678, 947, 949 ff., 955, 979, 1082, 1086, 1132

Sonderstaatsanwaltschaft Koblenz 77

Sonnenstein (Tötungsanstalt) 504, 526, 530

Sorau (Heilanstalt) 512

Sowjetunion $631,693,826,828 \mathrm{f} ., 958,1052$, 1128

Sozialdemokratie 12f., $17 \mathrm{f}$., 29, 32, 832

Sozialdemokratische Partei Deutschlands (SPD) 19, $25 \mathrm{f} ., 31,35,48 \mathrm{f} ., 53,59,62 \mathrm{f} ., 75$, $125,127,144,211,213,224,230,233,465$, 564,600
Spanischer Bürgerkrieg 781

SS (Schutzstaffel) 3, 16, 67, 82, 153, 209, 212, 216, $218 \mathrm{ff}$., 224, 227, 233, 235, 249f., 269, 303, 313, $320 \mathrm{ff} ., 331,338 \mathrm{f} ., 343 \mathrm{ff} ., 354$, 362 f., 384, 393 ff., 397, 407 f., 412, 415, 427 f., 434 f., 437 f., 465 ff., $474,478,485$ f., $532 \mathrm{f} ., 549,558,572,593,632 \mathrm{ff} ., 642$, $653 \mathrm{ff} ., 658 \mathrm{ff} ., 663 \mathrm{ff} ., 703,706,784,787$, $797,806,825,831,847 \mathrm{ff} ., 870,949,969$, 980, 1032, 1091, 1099, $1119 \mathrm{ff} ., 1123 \mathrm{ff}$.

- Leibstandarte Adolf Hitler 435, 442, 447, 478

- Mißhandlungen $348 \mathrm{ff}$., $362 \mathrm{ff}$., $375 \mathrm{ff} ., 381$, $388,393 \mathrm{f} ., 403 \mathrm{f} ., 458,463,478,547,632 \mathrm{ff}$., $648 \mathrm{ff} ., 654,1120,1125,1127$

- Morde s. auch „Erschießung auf der Flucht", Exekutionen und Polizei, Exekutionen 363, $375 \mathrm{f} ., 435 \mathrm{ff}$, $442 \mathrm{f}$., $455 \mathrm{ff}$., 547 , $634 \mathrm{ff}$., $646 \mathrm{ff} ., 655,1125,1127$

SS- und Polizeiführung 5, 67, $81 \mathrm{f} ., 224,322$, 366, 456, 479, 485f., 546, 647, 653, 667, $675 \mathrm{f} ., 681 \mathrm{ff} ., 719,727,737 \mathrm{f} ., 740 \mathrm{f} ., 744$, $889,998,1003,1017,1055,1058,1102$, $1127 \mathrm{f}$

- Kritik an Gerichtsurteilen 540, 621, 658-663, 693, 879, 888, 1100 f., 1141

SS- und Polizeigerichtsbarkeit 5, 366, $377 \mathrm{f}$, 412,654 ff., 682, 718, 944, 1127

Staatsanwälte 121, 214f., 217, 238, 263, 266 ff., 281, 290 ff., 294f., 299, 301 f., 304, $333,337,346 \mathrm{f} ., 366 \mathrm{f} ., 531,561,622,728 \mathrm{ff}$., 879, $884 \mathrm{f} ., 949,1044,1047,1051,1060 \mathrm{f}$., 1066f., 1096, 1098, $1102 \mathrm{f} ., 1105 \mathrm{f} ., 1121 \mathrm{f}$.

- Berichtspflicht s. auch Staatsanwaltschaft, Mitteilungen in Strafsachen 340, $346 \mathrm{f}$., 432, 488, 490, 496, 511, $519 \mathrm{ff} ., 527,530 \mathrm{f}$., $582,729,742,861,885,914,954 \mathrm{f} ., 1061$, 1066, 1078 f., 1085 f., 1091 ff., 1105, 1123 , 1141

- Entscheidung über Gerichtszuständigkeit 942 f., 950 ff., 978, 1134

- jüdische 126, $128 \mathrm{ff} ., 140 \mathrm{ff} ., 157,166 \mathrm{f}$., $169 \mathrm{ff} ., 323,1117$

- preußische 457, 462, 472f., 475

- Stellung im Strafverfahren s. unter Strafverfahrensrecht

- Zahl der $298 \mathrm{f}$.

Staatsanwalt 785, $801 \mathrm{ff} ., 814,835,877,910$, $914,933,936,966,980,993,1028,1030$, $1088 \mathrm{ff}$.

- „Herr des Vorverfahrens“ 986, 1005, 1012, $1018,1021,1032,1065,1135$

Staatsanwaltschaft(en) $295,324,332,345,397$, $401,405,408$ ff., 427,430 f., 438 ff., 444 , $447,457,462,472$ f., 474 f., 487 ff., 496,514 , 516 ff., 540, 542, 549, 554, 557, 560, 584, 
587, $593 \mathrm{ff}$., 598, 610 f., 613 f., $627,633 \mathrm{ff}$., 656, 681 f., 710, 713 ff., 719, 722, 724, 808 f., $815,825,831,861,863,907,935,947,976$, 1074, 1079, 1085, 1092, 1123, 1127, 1141

- Altona 541

- Ansbach $404 \mathrm{f}$.

- Aschaffenburg $394 \mathrm{f}$.

- Augsburg 400

- Bamberg 395

- Bayreuth 386

- Berlin 656, 704, $708 \mathrm{f} ., 848$

- Bonn 540

- Braunschweig 461, 463

- Bremen 593

- Breslau 462 ff., 467 f., 543 f., 744

- Chemnitz 525

- Darmstadt 881

- Detmold 622

- Dresden $369 \mathrm{ff} ., 508,525$

- Düsseldorf $710 \mathrm{ff} .$, 715, 717

- Duisburg $710 f$.

- Freiburg i.Br. 595

- Glogau 480

- Graz 581

- Hamburg 375f., 705, 707, 717, 884

- Hannover 634

- Hirschberg $460 \mathrm{ff}$.

- Innsbruck 581

- Kaiserslautern 383f., 487

- Koblenz 348

- Landshut 382, 575, $578 \mathrm{f}$.

- Leipzig 517, 529,745

- Magdeburg 598

- Mosbach (Baden) 493, 530

- München I 406, 435, $439 \mathrm{ff} ., 447,459,464$, $606,633,636,639,644,695$

- München II 412, 438, 442, 459, 634 ff., 639 ff., 645

- Münster 519

- Neisse 734

- Nürnberg-Fürth 386 ff., 393

- Osnabrück 363 ff., 459f., 468, 478, 646

- Passau 464

- Potsdam 443 ff., 511, 662

- Ravensburg 507f., 510, 519

- Regensburg 381

- Saarbrücken $491 \mathrm{f}$.

- Schweidnitz 462

- Stuttgart 880

- Traunstein 479

- Tübingen $665 \mathrm{f}$.

- Ulm 420

- Weiden 655

- Wels $519 \mathrm{f}$.

- Wien 581

- Wuppertal $354 \mathrm{ff} ., 360,362,531,733 \mathrm{f}$.
- Zwickau 518

- Absicht der Übernahme durch die Polizei 597 f., 998 f., 1020 f., 1125

- Mitteilung an die Polizei über Haftentlassung $587 \mathrm{ff}$., $612 \mathrm{ff}$., $620,622 \mathrm{f}$., 1025, 1096, 1126

- Mitteilungen in Strafsachen 356, $402 \mathrm{f}$., 408, 430 f., 468, 488, 593, 629, 1093

- Verhältnis zur Polizei $1018 \mathrm{ff} ., 1022 \mathrm{ff}$., 1135

- Zahl der 119

Staatsautorität $17,33,40,65,89,139,148$, 339 f., 342, 346, 359, 381, 384, 386, 388 f., $392,420,428,473 \mathrm{f}$., 586, 625, 642, 667, $669,680,706,740,872 \mathrm{f}$., $1067,1120 \mathrm{ff}$., 1128

Staatsgerichtshof für das Deutsche Reich 24, $34 \mathrm{f} ., 60$

"Staatskonkurs" s. Staatsprüfung, juristische

Staatsnotstand 418, 423, $438 \mathrm{f}$., 442, $448 \mathrm{ff}$., 452 ff., $466 \mathrm{f}$., 471, 483, 563, $704 \mathrm{ff}$., 711, $716,1122,1126$

Staatsnotwehr s. Staatsnotstand

Staatspolizei(leit)stelle(n) 456f., 485, 487, 489, 548,554 f., $560 \mathrm{ff}$., $565 \mathrm{ff}$., $573,585,588$, $592 \mathrm{ff}$, 606,609 f., $612 \mathrm{ff} ., 617 \mathrm{ff} ., 623,627 \mathrm{ff}$., $659,676 \mathrm{f}$., $690 \mathrm{f}$., $700,702,710,712,716 \mathrm{f}$., 871, 1093, 1127

- Berlin 696, 704

- Bielefeld 622

- Breslau 543, 696

- Dresden 620

- Düsseldorf 355, 479, 588, $704 \mathrm{f}$.

- Erfurt 588,613

- Halle 588, 613, 685

- Hamburg 604, 623, 628, 659, 705, 707, 712

- Karlsruhe 582

- Klagenfurt 596

- Köln 456

- Königsberg 617

- Magdeburg 588,613

- München 236, 407, 620, 624, 718

- Münster 565

- Oppeln 544

- Osnabrück 363, 365 f.

- Potsdam 592, 594

- Saarbrücken 491

- Stettin $348 \mathrm{ff}$, 352, 601, 620

- Stuttgart 507, 585, 612

- Trier 283

- Berichte über ungenügende Gerichtsurteile 659

- Strafverfolgung nur auf Antrag der $904 \mathrm{f}$.

Staatsprüfung, juristische $12,14,94,106$, 109 f., 140 f., 144, 153 f., 216, 234, 267, 293, $299 \mathrm{ff} ., 312,315 \mathrm{f}$., $318 \mathrm{f}$. 
Staatsraison $29 \mathrm{f}$., $40,47,64,69,80,451,474$, $830,839,1113$

Staatssekretär

- im bayerischen Justizministerium („Staatsrat") 20f., 23 f., 34, 55, 231, 239

- im preußischen Justizministerium $101 \mathrm{f}$., $123,125,132,219,222 \mathrm{f} ., 225,322 \mathrm{f} ., 325$, 330, 346, 431, 446f., 453, 754 f., 757

- im Reichsjustizministerium 71, 83, 86, 92, $102,106,118,123,130,135,137,174,213$, 219,241 f., 244, 246 f., 252, 260, 274, 303, $308 \mathrm{f} ., \quad 325 \mathrm{f} ., 330,367,480,505$, $510 \mathrm{f}$., $520 \mathrm{ff}$., $526 \mathrm{f}$., 529, 531, 561, 563, 602, 611, $621,664,670,685,687,728,732,750,754$, $793,805,824,828,895,902,924,927 \mathrm{f}$., $946,994,1002,1015,1071,1073,1095$, $1101,1117,1123,1127$

"Staatsstreichpläne“"

- v. Papens 58f., 62

- Röhms 433f., 436, 443, 449, 454, 476, $1121 \mathrm{f}$.

"Staatsverbrecherverordnung“ (Entwurf) 687, $820,918 \mathrm{ff}$.

Stahlhelm 64, 193, 285, 320, 331, 383, 833, 1113

Standesämter 504, 515, 869, 871, 873 f., 876, 878

Standgericht(e) 435, 678, $955 \mathrm{f} ., 980,1132$, 1134

- KZ Dachau 637

- München $18 \mathrm{f}$.

Stargard (Pommern) 348

Stellvertreter des Führers der NSDAP 115, $169,177,179,182,185 \mathrm{ff} ., 191,193,203$, 206 f., 209 f., 215 ff., 250,255 ff., 262,264 f., 268, $273 \mathrm{ff} ., 279,282,319,329,355,371$, $377,456,479,488$ f., 494, 496, 670, 689, 748 f., 756, 767, 769, 795, 797, 799 ff., 806 f., $811,813 \mathrm{f}$., 816, 818, 820, 833, 849, $852 \mathrm{ff}$., $874,877,882,885$ f., 888 ff., 903,920 , 962 ff., 967, 976, $993 \mathrm{f}$., 1011, 1015, 1017, $1045,1068,1078,1118,1135$

- Zustimmung zu Strafverfahren 809, $813 \mathrm{ff}$., $819,833,890,922,1046 \mathrm{ff} ., 1092,1123$, 1135

Stendal (Bezirk Merseburg) 284

Sterilisation $837,844,870$

Stetten im Remstal (Heilanstalt) 513

Stettin 184, 226, 271, $348 \mathrm{ff} ., 1120$

"Steuerung“ der Rechtsprechung s. Lenkung

Stillstand der Rechtspflege (i.S. des $\S 245$ ZPO) 124, 322

Strafbefehl(e) 991, 1070, 1084, 1090, 1137

- Zahl der 1070

Straffreiheit $324 \mathrm{ff} ., 329 \mathrm{ff} ., 334 \mathrm{ff} ., \quad 347,353$, $367,376,428,431,448,451$ f., 465, 491,
639, 642 f., 813, 835 f., 845, 984, 1006, 1047, $1086,1119 \mathrm{f}$.

- durch Aktenverlust 357, 639

- Erlaß v. 7.6.1939 335

- Gesetz v. 20.12.1932 325f., 337

- Gesetz vom 7.8.1934 335f., 345, 353, 361, 370, 374, 376f., 379, 411, 432, $464 \mathrm{f}$., 467, 475, 478f., 645, 705, 707, 1120

- Gesetz vom 23.4.1936 335, 469

- Gesetz vom 30.4.1938 335

- Verordnung v. 21.3.1933 324-328, 330, $332,397,1119$

- Verordnung v. 2.8.1933 332, $380 \mathrm{ff}$., 388, $393,411,639,642$

Strafgefangenenlager s. unter Strafvollzugsanstalten

Strafgesetzbuch (StGB) 19, 27, 29, 84, 104, $113,118,200,204,330,362,370,377,400$, 409, 411, 418, 452f., 459, 466, 494, 496, 499, 513, 516f., 519, 556, 619, 621, 638, $660,667,687,692 \mathrm{f} ., 703,711,713 \mathrm{f} ., 716$, $720 \mathrm{f}$., $723,728,730 \mathrm{f}$., $733,735,737,740 \mathrm{f}$., 743 f., $752,755,760$ f., $765 f$., 769 f., 773 f., 776, $778 \mathrm{f} ., 784 \mathrm{ff}$., $791 \mathrm{f}$., $794 \mathrm{f}$., 798,800 , $805,808,813,816$ f., 822 f., 827 f., 830 , $834 \mathrm{ff}$., $838 \mathrm{ff}$., $844,848,850 \mathrm{ff}$., $856,861 \mathrm{ff}$., 865 f., $880,882,886$ ff., 893 f., 896 f., 902 , 907, 910, $914 \mathrm{f}$., 917 f., 921 ff., 925, 950 , 1026, 1046f., 1049, $1060 \mathrm{f} ., 1072,1076$, $1128,1130,1134$

- angekündigter Entwurf der NSDAP 768

- Entwurf von 1922760,780

- Entwurf von 1925 760, 838, 1056, 1128

- Entwurf von 1927 753, 760, 838, 1056, 1128

- Entwurf erster Lesung vom Januar 1935 $766,850,865,985$

- Entwurf vom Dezember 1936 735, 749, 773-791, 792 ff., 846f., 866, 914 f., 918, 928 , 951, 977, 1025, 1129

- Entwurf i.d.F. vom Juni 1938 737, 753, 806, 810 ff., 898, 912, 917, 1025 ff., 1033 , $1047 \mathrm{f}$.

- Entwurf i.d.F. vom Dezemer 1939740 f., $807,820 \mathrm{f}$.

- österreichisches 805, 811

- sowjetisches 850

„Strafgewalt“ (Strafbann) 977 ff., 1090, 1134

Strafkammer

- große 932, 934, 938f., 941, 953, 976, 1062, 1096

- kleine 933, 938, 944, 976, 982

Strafminderung $28,404,779,842,914,918$, 1080, 1084, 1100, 1103

Strafprozeßkommission

- („Große“) Amtliche 72, 697, 736, 741, 931, 
$939 \mathrm{ff} ., 951,953,980 \mathrm{f} ., \quad 1008,1011 \mathrm{f}$., 1015-1046, 1049f., 1055, 1069, 1075, 1082, $1098 \mathrm{ff}$.

- „kleine“ 232, $931 \mathrm{f}$., 935, 940, 946, $981 \mathrm{ff}$., 988, $993 \mathrm{f} ., 1003,1012,1015,1019,1035$, 1038

Strafprozeßordnung (StPO) 31, 321, 341, 375, 385, 387 f., 391, 393, 402, 405, 408 f., $415 \mathrm{f}$., $427,429 f ., 452,519,537,549,557,560$, 584 ff., 588,592 ff., 597 f., $604,606,633$, 653, 692, 700 f., 714, 716, 729, 794, 843, $859,881,888,958,979$ f., 982, 986f., 991, $1003 \mathrm{ff} ., 1014,1019,1022 \mathrm{f} ., 1030,1034$, 1050 ff., 1055,1057 ff., 1063 f., 1066, 1068, $1070,1078,1083,1088,1090,1100$, $1106 \mathrm{ff} ., 1110,1121,1123 \mathrm{f} ., 1134 \mathrm{f}$., 1142

- österreichische v. 23.5.1877 $1084 \mathrm{f}$.

Strafprozeßrecht s. Strafverfahrensrecht

Strafrahmen 1103, 1126, 1129

Strafrecht $86,106,113,242,722,737,741$, 748, 758, 760, 791, 798, 865 ff., 905, 921, $931,990,1047,1091,1112,1135$

- Ausscheidung des Täters aus der Volksgemeinschaft $924 \mathrm{f} ., 1131$

- „autoritäres“ 765, 790, 847, 855 f., 860, 901, $924 \mathrm{f}$., 956, 1128, $1130 \mathrm{f} ., 1137,1144$

- Einführung des deutschen in Polen 915

- Erfolgsstrafrecht 765, 775, 914, 925

- erhöhter Schutz für „Staatsträger“ und Prozeßbeteiligte $831 \mathrm{f}$.

- Gefährdungsstrafrecht 763 ff., 782, 843

- Geltungsbereich 780,915

- Gesetzgebung 822-925, $1128 \mathrm{ff}$.

- Gesetzgebung zum politischen $822 \mathrm{ff}$., $831 \mathrm{ff} ., 1128$

- Gesinnungsstrafrecht 458, 775

- nationalsozialistisches 855,925

- österreichisches 885

- Reform 6, 242, 259, 494, 497 ff., 621, 687, 693, 731, 740f. 746, 748f., 752-822, 824, $833 \mathrm{ff}$., 846,851 ff., $861,864,886 \mathrm{ff}$., 912 , $919 \mathrm{ff} ., 925,981,994,1002,1046,1049$, $1128 \mathrm{ff}$.

- Reformkontinuität 773, 789f., 838, 843, $1128 \mathrm{f}$.

- Tätertypenstrafrecht 908f., 977, 1000, 1005

- Verletzungsstrafrecht 763

- Willensstrafrecht $722 \mathrm{f}$., $763 \mathrm{ff} ., 775,778$, 782, 788, 892, 894, 910, 914, 925, 977, 1005,1129

Strafrechtliche Fortbildungswoche in Jena 1101

Strafrechtskommission $71,104,232,234,285$, 498, 546, 556, 731, 753-773, 778, 786f., 789 ff., $796,804,810,819,824$ f., 844, 847 ,
850 f., 856 ff., 864 ff., 895 f., 985, 993, 1015, $1129 \mathrm{f}$.

Strafregister $86,107,470,993$

Strafsache(n), politische $s$. Tatbestand, politischer

Strafverfahren s. auch Hauptverfahren, Vorverfahren und Wiederaufnahme des Verfahrens $743,948,1049$

- „Auflockerung“ 983, 988 f., 995 f., 1001, $1003,1045,1135 \mathrm{ff}$.

- autoritäres $983 \mathrm{f}$., 995, 1001, 1137

- beschleunigtes s. Schnellverfahren

- Beschleunigung 982, 995, 1003, 1045, 1068 f., 1135

- Beteiligung eines Kommissars der NSDAP $999 \mathrm{f}$.

- gegen Abwesende 771, $982 \mathrm{f} ., 991 \mathrm{f.,}$ 1013, 1015, 1052 f., 1066 ff., 1069, 1136

- Mündlichkeit und Unmittelbarkeit 1067

- nur mit Zustimmung des Stellvertreters des Führers 809, $813 \mathrm{ff} ., 819,833,890,922$, $999 f ., 1046 \mathrm{ff} ., 1092,1123,1135$

- Reform 954, 980 ff., 1032 ff., 1046, 1049, 1053, 1058, 1065, $1068 \mathrm{ff} ., 1090 \mathrm{f} ., 1134 \mathrm{ff}$.

- Reformkontinuität 1090

- „Richtlinien“" s. dort

- Täter-Strafverfahren 1000

- Vereinfachung im Kriege $1068 \mathrm{ff} ., 1090 \mathrm{f}$., $1136 \mathrm{f}$

- Verhinderung durch „Aktenverlust“ 357, 639,1119

Strafverfahrensordnung (StVO) 793, 803, 808 f., 813, 817, 931, 951, 1039, 1046, 1049

- Entwurf von 1920981

- Entwurf 1. Lesung v. 15.12.1934 $931 \mathrm{f}$., $981,984,986$

- Entwurf v. 11.3.1935933 ff., 937

- Entwurf v. 27.2.1936 736, 813, 937 ff., 951, 980, 985 ff., 993 f., 1016, 1020, 1034, 1036 f., $1044 \mathrm{f} ., 1047 \mathrm{f} ., 1052,1057,1059 \mathrm{f} ., 1065$, $1068,1090,1134$

- Entwurf v. 1.5.1939 $942 \mathrm{ff} ., 974,976 \mathrm{f}$, 1017,1023 ff., $1028 \mathrm{ff}$., 1032-1045, 1047, 1050, 1055, 1057, 1059f., 1068, 1070, 1072, $1074,1076,1082,1084,1088,1090,1123$, 1134,1136

Strafverfahrensrecht $6,29,567,570,596,659$, $701,758,819,847,855,931,944,954,974$, 980 ff., 1049, 1057, 1091 f., 1112, 1138, 1145

- Ablehnung des „Parteiprozesses“ $980 \mathrm{f}$., 998

- Bestätigung der Urteile durch die Justizverwaltung 1141

- Bestätigung der Urteile durch Hitler 997, 1006, $1076 \mathrm{f} ., 1140$

- Gesetzgebung 1049-1091 
- Grundsātze und Ziele der Reform 982 ff., 995, 1003, $1032 \mathrm{f}$.

- nationalsozialistisches $1001 \mathrm{f} ., 1008,1018$, 1045f., 1048, 1137

- präventive Befugnisse der Polizei $1021 \mathrm{ff}$., $1025 \mathrm{ff} ., 1058,1135$

- Reformkontinuität 992, 996, 1016, 1045

- Stellung des Gerichtsvorsitzenden s. Gerichte, Rechte des Vorsitzenden

- Stellung des Staatsanwalts $980 \mathrm{ff}$., $985 \mathrm{ff}$., 992, $998 \mathrm{f} ., 1001,1004 \mathrm{ff} ., 1012 \mathrm{f} ., 1018 \mathrm{ff}$., 1032 f., 1047, 1063 ff., 1089 f., 1135 f., 1145

Strafvollstreckung $610 \mathrm{ff}, 618,729,734,785$, 935, 990, 1053, 1066f., 1074f., 1078, 1083, 1088, 1094, 1132

Strafvollstreckungsordnung $605,610,793$

Strafvollzug 7, 86, 91, 106f., 113, 242, 247, 268, 344, 364, 576f., $579 \mathrm{f} ., 613 \mathrm{ff} ., 617$, 621 f., $652,713,742,776$ f., 838, 840, 842, 1039

- Tageskosten 735

- verschärfter 583

Strafvollzugsanstalt(en) 296f., 319, 368, 547, 573-583, 613 ff., 624, 629f., 647, 675, 681, $710,719,729$ f., 733 ff., 742 f., 840, 1127

- Bautzen I 368

- Bernau (Chiemsee) 619

- Brandenburg 352

- Bruchsal 615

- Freiburg i.Br. 615

- Fuhlsbüttel 374

- Gollnow 352

- Landshut 575, 579

- Laufen 619

- Lübeck-Lauerhof $577 \mathrm{f}$.

- Mannheim-Heidelberg 615

- Naugard 352

- Stadelheim 434 ff., 442, 459, 575

- Strafgefangenenlager Emsland 364, 464, 735, 742

- Wohlau 675

- Zahl der 119, 296

Strafvollzugsbeamte $107,155,290,296 \mathrm{ff}$., $302,408,615,729$

- jüdische 128, 155

Strafvollzugsordnung 578, $580 \mathrm{f.,} \mathrm{646,1127}$

Strafzumessung $19,28,200,621,722,730$, $732,763,775,778,790,879,888,900,933$, $979,982,1027,1036,1074,1097 \mathrm{f.}, 1100 \mathrm{ff}$., $1105 \mathrm{ff} ., 1126,1137,1141,1143$

Straßenraub 908, 913

- mittels Autofallen 806, 897 ff., 952, 1110, 1140

- mittels Autofallen, Zahl der Todesurteile 952

Streikverbot $762,782,801,919$
Studentenwerk München 439

„Der Stürmer“ 176, 231, 394f., 663, 671,870

Stuttgart 56, 91, 109, 111, 114, 835, 1017

Sudetendeutsche Partei (SdP) 278, 286

Sudetenland 179, $277 \mathrm{f}$., 280, 286f., 303, 657, $660,808,817,1026$

Südamerika 865

Sylt 201, 257, 330, 431, 831

Tātertyp 786, 839, 843, 901, 906 ff., 911, 913 f., 925,1130

"Tagesbußen“ 789, 1047

Tatbestand 712, $720 \mathrm{f}$.

- exakter 765 f., $810,857,925$

- krimineller (nichtpolitischer) 20, 197, 329, 408, 410, 418, 470, 535f., 547, 550, 591, $733,925,951,956,1087,1107,1120,1122$

- neuer 417, 550, 690 f., 761 ff., 781 ff., 801 , $823 \mathrm{ff} ., 836,845,889,900,907,913,918$, $1052,1128 \mathrm{ff}$.

- objektiver $19,40,61,324,774,813,889$, 905, 999, 1143

- politischer 19, 26, 186, 197, $324 \mathrm{ff} ., 332$, 334, 336, 343, 346 f., 406 ff., 410 f., 470,476 , 488, 496, 519, $535 \mathrm{f}$., $584 \mathrm{ff} ., 593,595,606$, $609,643,790,862,897,925,939,949,951$, 1050, 1052, 1091 f., 1096, 1107, 1120, 1123, $1130,1132,1141,1143$

- subjektiver 19, 324, 491, 660, 774, 813, 889

- unbestimmter 862, 889, $898 \mathrm{f}$., 901, $906 \mathrm{ff}$., 914, 1130 f., 1138

Technische Nothilfe 251, 254, 787, 833

Tegernsee (Oberbayern) 571

Teltower Bankräuber $679 \mathrm{ff} ., 685,911 \mathrm{f} ., 1110$

Teupitz (Heilanstalt) 512

Thüringen 32, $98 \mathrm{f}$., 109, 112, 114, 228, 239, $246,344,660,945$

Tierschutz 837

The Times 75

Tittling (Niederbayern) 382

Todesstrafe(n) $61,328,333,365,415,457$, $517,521,536,597,637,646 \mathrm{f}$., $677 \mathrm{f} ., 680 \mathrm{f}$., $687,690,693,746,761 \mathrm{f} ., 771,776,779$, $781,786,799$ ff., $804,806,823$ ff., 829 , 831 f., 845 f., $855,864,886$ f., 892 ff., 897 ff., 906 ff., 910 f., 916 ff., 921,923 f., 957, 977 , $997,1052,1071,1079$ f., 1089, 1103 f., 1110 , $1127 \mathrm{f} ., 1131,1134,1143$

- Ausweitung 790, 900, $1129 \mathrm{ff}$.

- Vollstreckung 776, 799, 826, $829 \mathrm{f}$, 900, 907, 1094

- Vollstreckung durch „Schierlingsbecher“ 776

Todesurteil(e) 18, $677 \mathrm{f}$., $685,687 \mathrm{f}$., 705,830 , 839, 855, 896, $898 \mathrm{f}$., 911, 946, 957, 965, 
1006, 1052, 1071, 1074, 1078 ff., 1094, $1128,1136,1143$

- wegen Abhörens von Auslandssendern $905 \mathrm{f}$.

- wegen „Rassenschande“ 909

- wegen Straßenraubs mittels Autofallen 952

- Zahl der vollstreckten 900

- Zahlen 900

„Trauma von 1918“ 901, 921, 1103, 1131

Treubruch $761,781,784,790,836,847,910$, 925

Tschechoslowakei 372, 641, 806

Tscheka 569

Tübingen 585, 766

\section{Überzeugungstäter 776}

Ulmer Reichswehrprozeß 956

Unabhängigkeit der Gerichte $69,77 \mathrm{f}$, 139 , $196 \mathrm{f} ., 343,381,672,680,935,997,999$, $1007,1038,1102,1133,1138,1142 \mathrm{ff}$.

Unabhängigkeit der Richter

- persönliche 49, $130 \mathrm{ff} ., 137,156,161,164 \mathrm{f}$., 190, 192 ff., 200 ff., $281,317,671$ f., $973 \mathrm{ff}$., 1110 f., $1118 \mathrm{f}$., $1133,1137 \mathrm{f} ., 1142,1144$

- sachliche 26, 40f., 49, $191 \mathrm{ff}$., $195 \mathrm{ff} ., 205$, $372,391,528,625,659,668,671 \mathrm{f}$., $730 \mathrm{f}$., 861, 983, 997 f., 1003 f., 1010, 1035, 1064, $1077, \quad 1100 \mathrm{ff} ., \quad 1105 \mathrm{f} ., \quad 1108 \mathrm{ff} ., \quad 1134$, 1138 ff., 1144

Unabsetzbarkeit des Richters s. Unabhängigkeit des Richters, persönliche

United States of America (USA) 894, 896

Unterbringung $516 \mathrm{ff} ., 1057 \mathrm{f}$., 1065

Untergöltzsch (Heilanstalt) 518

Unterhaltspflicht $740 \mathrm{f}$., 782

Unterlassung $763,765,775,789,854,886 \mathrm{ff}$., $892 \mathrm{f} ., 917$

Unterschlagung $423,440,762,764,788$, 836

Unterstaatssekretär s. unter Reichsjustizministerium

Untersuchung, zwangsweise körperliche 1059

Untersuchungshaft $322,325,564,574,577$, 579 f., 582 ff., 599 f., 603 ff., 607,609 ff., 661 , $685,704,707,709,740,883,915,948,965$, $983,986,989,1000,1014,1040,1044$, 1050, 1057, 1059f., 1066, 1075, 1078, 1081, $1088,1096,1126,1135$

- Erweiterung der Gründe 1065 f., 1136

- Vermeidung durch finanzielle Sicherheitsleistung 984, 989, 1032, 1057

Unversetzbarkeit des Richters s. Unabhängigkeit des Richters, persönliche

Unzurechnungsfähigkeit s. Zurechnungsunfähigkeit

Urheberrecht $665,787,927,1132$
Urteil(e)

- Bestätigung der s. unter Analogie und unter Strafverfahrensrecht

- Korrektur durch die Polizei s. unter Schutzhaft

- Kritik durch die Polizeiführung s. unter SSund Polizeiführung

- Zahl der Strafurteile 953, 1143

„Urteilsrüge“ 943, 1009, 1011

Vaterländische Front 261, 278, 285, 288

Vaterländische Verbände $17,22,27,29 \mathrm{f}$., 32 f., 35, 43 f., 46, 48, 51

Verdächtigung, falsche $809,814,816,836$

Verdunkelungsgefahr 561, 585f., 588, 635, 989, 1057, 1065

Verdunkelungsverbrechen $681,689,907 \mathrm{ff}$., 1103,1131

Vereinfachungsverordnungen für die Strafrechtspflege v. 1.9.1939, 13.8.1942, 29.5.1943 s. unter Verordnungen

Vereinigte Schlesische Granitwerke $\mathrm{GmbH}$ 695

Vereinigung nationaler Anwälte 130

„Verfahrensrichter“ 1005

Verfassung

- bayerische 20, 24f., 29f., 48, 332

- Danziger 863

- nationalsozialistische $799,919,996,1018$, 1076, 1124, 1129

- preußische 330, 545

- Reichsverfassung von 1871980

- Weimarer 20, 29, $32 \mathrm{f}$., 37, $48 \mathrm{f}$., $57 \mathrm{ff}$., 62 , $66,85,133,211,535 \mathrm{f} ., 543 \mathrm{f} ., 548,827 \mathrm{ff}$., 834, $946 \mathrm{f}$., 959, 1054f., 1076, 1113, 1115, $1143 \mathrm{f}$.

Verhältnismäßigkeit der Mittel 454

Verjährung $27 \mathrm{f}$., $834 \mathrm{ff}$., 839

Verkehrsstrafsachen 1050, 1095

Vermögenseinziehung 776f., 845, 864, 870, 936, 1033, 1066

„Vernichtung durch Arbeit“ 534, 745

Verordnung (in zeitlicher Reihenfolge:)

- betreffend die einstweilige Versetzung der unmittelbaren Staatsbeamten in den Ruhestand v. 26.2.1919 222

- des Reichspräsidenten auf Grund des Artikels 48 Abs. 2 der Reichsverfassung, betreffend die zur Wiederherstellung der öffentlichen Sicherheit und Ordnung im Reichsgebiete ... nötigen Maßnahmen v. 13.1.1920

- über Gerichtsverfassung und Strafrechtspflege v. 4.1.1924 s. Emminger-Novelle

- des Reichspräsidenten zur Sicherung der Haushalte von Ländern und Gemeinden v. 24.8.1931 60 
- zur Sicherung von Wirtschaft und Finanzen und zur Bekämpfung politischer Ausschreitungen v. 6.10.1931946f.

- preußische Sparverordnung v. 23.12.1931 161

- des Reichspräsidenten über Maßnahmen auf dem Gebiete der Rechtspflege und Verwaltung v. 14.6.1932 1061

- des Reichspräsidenten, betreffend die Wiederherstellung der öffentlichen Sicherheit und Ordnung im Gebiet des Landes PreuBen v. 20.7.1932 59f.

- des Reichspräsidenten, betreffend die Wiederherstellung der öffentlichen Sicherheit und Ordnung in Groß-Berlin und Provinz Brandenburg v. 20.7.1932 60

- der Reichsregierung über die Bildung von Sondergerichten v. 9.8.1932 946

- des Reichspräsidenten gegen politischen Terror v. 9.8.1932 61, 326, 828

- der Reichsregierung über die Aufhebung der Sondergerichte v. 19.12.1932 63, 946

- des Reichspräsidenten zur Erhaltung des inneren Friedens v. 19.12.1932 63

- des Reichspräsidenten zum Schutze des deutschen Volkes v. 4.2.1933 537, 823, 1050, 1070

- des Reichspräsidenten gegen Verrat am deutschen Volke und hochverräterische Umtriebe v. 28.2.1933 $823 \mathrm{f}$., $844 \mathrm{f}$., 900, 958, 960, 1051

- des Reichspräsidenten zum Schutz von Volk und Staat v. 28. 2.1933 s. Reichstagsbrandverordnung

- des Reichspräsidenten zur Beschleunigung des Verfahrens in Hochverrats- und Landesverratssachen v. 18.3.1933 958, 960, 1051

- des Reichspräsidenten über die Gewährung von Straffreiheit v. 21.3.1933 s. unter Straffreiheit

- des Reichspräsidenten zur Abwehr heimtückischer Angriffe gegen die Regierung der nationalen Erhebung v. 21.3.1933 s. auch Heimtückeverfahren 327, 476, 633, 824 f., 832 f., 845,946 f., 950

- über die Bildung von Sondergerichten v. 21.3. 1933 327, 946 ff., 988, 1052, 1066

- über die Zuständigkeit der Sondergerichte v. 6.5.1933 950

- zur Durchführung der Gesetze über die Zulassung der Rechtsanwaltschaft und zur Patentanwaltschaft v. 20.7.1933 159

- bayerische, über die Gewährung von Straffreiheit v. 2.8.1933 s. unter Straffreiheit

- zur Durchführung der Gesetze über die Zu- lassung zur Rechtsanwaltschaft und Patentanwaltschaft v. 1.10.1933 163

- über die Vereidigung der Beamten und der Soldaten der Wehrmacht v. 2.12.1933 206

- über den Vollzug von Freiheitsstrafen v. 15.5.1934 108

- zur Vereinheitlichung der Zuständigkeit in Familien- und Nachlaßsachen v. 31.5.1934 108

- über den Volksgerichtshof v. 12.6.1934 961,971

- über den Volksgerichtshof v. 29.6.1934 962, 966

- über die Reichsnotarkammer v. 17.7.1934 108

- zur Vereinheitlichung und Verbilligung der Verwaltung v. 19.7.1934 105

- über die Zuständigkeit der Sondergerichte v. 20.12.1934 950

- des Reichsjustizministers über das Verfahren in Gnadensachen v. 6.2.1935 (Gnadenordnung) 335

- über den weiteren Ausbau des Gemeinschaftslagers Hanns Kerrl v. 9.3.1935 304

- zur vorläufigen Regelung des Dienststrafrechts im Bereich der Justizverwaltung v. 15.3.1935 119

- zur einheitlichen Regelung der Gerichtsverfassung v. 20.3.1935 119, 317, 971, 975

- zur Vorbereitung der Überführung der bisherigen Landesjustizbeamten in die Reichsbesoldungsordnung v. 23.3.1935 118

- über die Laufbahn für das Amt des Richters und des Staatsanwalts v. 29.3.1935 119, 316, 975

- zur Durchführung des Gesetzes zur Sicherung der Einheit von Partei und Staat v. 29.3.1935 490, 797

- über den Volksgerichtshof v. 22.8.1935968

- über die Zuständigkeit der Sondergerichte v. 24.9.1935 950

- zum Reichsbürgergesetz v. 14.11.1935 163, 169 f., 181, 189, 770, 877 f.

- zur Ausführung des Gesetzes zum Schutze des deutschen Blutes und der deutschen Ehre v. 14.11.1935 878f.

- über den Waffengebrauch der Wehrmacht v. 17.1 .1936

- über die Zuständigkeit der Sondergerichte v. 5.2.1936 950

- über die Gestaltung des Hoheitszeichens des Reiches v. 7.3.1936 1124

- zur Durchführung des Gesetzes über den Volksgerichtshof und über die fünfundzwanzigste Änderung des Besoldungsgesetzes v. 18.4.1936 968, 971 
- zur Durchführung des Deutschen Beamtengesetzes v. 29.6.1937 191, 203, 205

- über die Altersgrenze der Beamten der Reichsjustizverwaltung v. 27.7.1936 292

- zur Anderung der Militärstrafgerichtsordnung und des Einführungsgesetzes zu ihr v. 5.9.1936 960

- zum Gesetz über die Wiedervereinigung Österreichs mit dem Deutschen Reich v. 18.3.1938 596, 1026

- über Angelegenheiten der Rechtsanwälte, Verteidiger, Notare und Patentanwälte in Österreich v. 31.3.1938 175, 181

- über die Einführung der Nürnberger Rassengesetze im Lande Österreich v. 20.5.1938 173

- zur Neuordnung des österreichischen Berufsbeamtentums v. 31.5.1938 173

- über die Einführung der Vorschriften über Hochverrat und Landesverrat im Lande Österreich v. 20.6.1938 597

- zum Reichsbürgergesetz v. 25.7.1938 178

- über das militärische Strafverfahren im Kriege und bei besonderem Einsatz v. 17.8.1938 s. Kriegsstrafverfahrensordnung

- über das Sonderstrafrecht im Kriege und bei besonderem Einsatz v. 17.8.1938 s. Kriegssonderstrafrechtsverordnung

- über Angelegenheiten der Rechtsanwälte, Rechtsanwaltsanwärter und Verteidiger in Strafsachen in Österreich v. 27.9.1938 181

- zum Reichsbürgergesetz v. 27.9.1938 $178 \mathrm{ff}$, , 185,188

- zur vorläufigen Ausübung der Rechtspflege in den sudetendeutschen Gebieten $\mathbf{v}$. 8. 10.1938278

- zum Erlaß des Führers und Reichskanzlers über die Verwaltung der sudetendeutschen Gebiete v. 22.10.1938 1026

- über Angelegenheiten der Patentanwälte im Lande Österreich v. 31.10. 1938188

- zum Reichsbürgergesetz v. 31.10.1938 187

- über die Erweiterung der Zuständigkeit der Sondergerichte v. 20.11.1938 678, 943, $951 \mathrm{f}$.

- über das Verfahren in Hochverrats- und Landesverratssachen v. 16.12.1938 831

- über die Beförderung zum Richteramt, zur Staatsanwaltschaft, zum Notariat und zur Rechtsanwaltschaft v. 4.1.1939 302

- über die Vorbildung und die Laufbahnen der deutschen Beamten v. 28.2.1939 216, 267 f., 292

- zur weiteren Úberleitung der Rechtspflege im Lande Österreich und in den sudeten- deutschen Gebieten v. 28.2.1939 277, 1085

- über die deutsche Gerichtsbarkeit im Protektorat Böhmen und Mähren v. 14.4.1939 279

- über die Laufbahn für das Amt des Richters und Staatsanwalts v. 16.5.1939 317

- über außerordentliche Rundfunkmaßnahmen v. 1.9.1939 597, 902 ff., 952, 955

- über Maßnahmen auf dem Gebiete der Gerichtsverfassung und der Rechtspflege v. 1.9.1939 (1. Vereinfachungsverordnung) 202, 317, 952 f., 974 ff., 977, 1069 ff., 1082, $1088 \mathrm{f} ., 1133$

- über Maßnahmen auf dem Gebiete des bürgerlichen Streitverfahrens, der Zwangsvollstreckung, des Konkurses und des bürgerlichen Rechts v. 1.9.1939 1069

- über die Vereinfachung der juristischen Staatsprüfungen v. 2.9.1939 301

- Kriegswirtschaftsverordnung v. 4.9.1939 901, 907, 952, 955

- gegen Volksschädlinge v. 5.9.1939 678, $681,683,693,906$ ff., 910 f., 913 f., 952,955 , 1086,1093 f., 1097,1102 ff., 1127,1130 f., 1141

- über die Behandlung von Ausländern v. 5.9.1939691

- zur Durchführung der Verordnung gegen Volksschädlinge v. 7.9.1939 907

- zur Durchführung der Verordnung über Maßnahmen auf dem Gebiete der Gerichtsverfassung und der Rechtspflege v. 8.9.1939 1069

- zur Durchführung des Gesetzes zur Änderung von Vorschriften des allgemeinen Strafverfahrens, des Wehrmachtstrafverfahrens und des Strafgesetzbuchs v. 17.9.1939 u. v. 11.12.1939 $1074 \mathrm{f}$., $1077 \mathrm{f}$.

- zum Schutz gegen jugendliche Schwerverbrecher v. 4.10.1939 741, 910f., 1086, 1131

- über eine Sondergerichtsbarkeit in Strafsachen für Angehörige der SS und für die Angehörigen der Polizeiverbände bei besonderem Einsatz v. 17.10.1939 654, 944

- über den Aufbau der Rechtspflege im Generalgouvernement v. 26.10.1939915

- über Sondergerichte im Generalgouvernement v. 15.11.1939915

- zur Ergänzung der Strafvorschriften zum Schutz der Wehrkraft des Deutschen Volkes v. 25.11.1939 820, 892, 906

- zur Änderung und Ergānzung des Strafrechts (Entwurf vom Dezember 1939) s. "Schwerverbrecherverordnung" und "Staatsverbrecherverordnung“" 
- über Verfahren in Hochverrats- und Landesverratssachen im Gebiet der bisherigen Freien Stadt Danzig v. 4.12.1939831

- gegen Gewaltverbrecher v. 5.12.1939680, 820, 843, 912 ff., 916 f., 952, 955, 1079, 1103,1131

- zur Ausübung der Rechtspflege im Gebiet der bisherigen Freien Stadt Danzig v. 5. 12.1939280

- zur Durchführung und Ergänzung der VO gegen Gewaltverbrecher v. 28.12.1939 914

- zur Ergänzung der Ersten Ausführungsverordnung zum Blutschutzgesetz v. 16.2.1940 $885 \mathrm{f}$.

- über die deutsche Gerichtsbarkeit im Generalgouvernement v. 19.2.1940915

- über die polnische Gerichtsbarkeit im Generalgouvernement v. 19.2.1940 915

- über die Zuständigkeit der Strafgerichte, die Sondergerichte und sonstige strafverfahrensrechtliche Vorschriften v. 21.2.1940 (Zuständigkeitsverordnung) 952, 954, 977 ff., 1083 ff., 1088 ff., 1134, 1137

- bayerische, über die Behandlung von polnischen Arbeitskräften v. 11.3.1940 691

- zur Durchführung der Verordnung über die Zuständigkeit der Strafgerichte, die Sondergerichte und sonstige strafverfahrensrechtliche Vorschriften v. 13.3.1940 1085, 1088

- über den Geltungsbereich des Strafrechts v. 6.5.1940 915

- über die Einführung des deutschen Strafrechts in den eingegliederten Ostgebieten $\mathbf{v}$. 6.6.1940 831, 915

- zur Durchführung der Fünften Verordnung zum Reichsbürgergesetz v. 12.6.1940 $186 \mathrm{f}$.

- zur Durchführung der Verordnung über außerordentliche Rundfunkmaßnahmen $\mathbf{v}$. 2.7.1940 u. 20.9.1940 904

- über die vorläufige Ausübung der Rechtspflege in den Gebieten Eupen, Malmedy und Moresnet v. 29.7.1940 831

- zur Ergänzung des Jugendstrafrechts v. 4.10.1940 910

- zur weiteren Ergänzung der Reichs-Rechtsanwaltsordnung v. 24.6.1941 702

- zur Durchführung des Gesetzes zur Änderung des Reichsstrafgesetzbuches v. 24.9.1941 728

- über die Strafrechtspflege gegen Polen und Juden in den eingegliederten Ostgebieten v. 4.12.1941 692, 905, 915, 1127, 1145

- zur weiteren Vereinfachung der Gerichtsverfassung, der bürgerlichen Rechtspflege und des Kostenrechts v. 16.5.1942979
- zur Durchführung des Deutschen Beamtengesetzes v. 29.7.1942 203

- zur weiteren Vereinfachung der Strafrechtspflege v. 13.8.1942 (2. Vereinfachungsverordnung) 979, 1070, 1083, 1086f., 1090

- über die Anrechnung von Wehrdienst bei der Zulassung zu den juristischen Staatsprüfungen v. 27.11.1942 301

- zur Durchführung der Verordnung zum Schutz von Ehe, Familie und Mutterschaft v. 18.3.1943 741

- zur Vereinfachung der Strafrechtspflege v. 29.5.1943 (3. Vereinfachungsverordnung) 979,1090

- dreizehnte, zum Reichsbürgergesetz v. 1.7.1943 185, 597, 886

- zur weiteren Anpassung der Strafrechtspflege an die Erfordernisse des totalen Krieges v. 13.12.1944 (4. Vereinfachungsverordnung) 980,1090

- über die Errichtung von Standgerichten v. 15.2.1945 980

Verreichlichung der Justiz $49 \mathrm{ff}$., $85 \mathrm{ff}$., $91 \mathrm{ff}$., $96 \mathrm{ff} ., 100 \mathrm{ff} ., 107,117 \mathrm{ff} ., 169,207,231,240$, $246,263,271,315,329,368,377,379,407$, $588,610,613,645,662,747,751,767,770$, $947 \mathrm{f}$., $969,1091,1115 \mathrm{ff}$.

Versailler Vertrag 16f., 25, 66, 834, 875, 1114

"Verschärfte Vernehmung" s. auch Aussagen, Erpressung der $359 \mathrm{ff} ., 370,391,646 \mathrm{f}$., 703-719, 785, 883, 1031, 1127

- Besprechung im Reichsjustizministerium v. 4.6.1937 $715 \mathrm{ff}$.

Versorgungsanwärter $295 \mathrm{f}$.

Versorgungsberechtigte der Justiz, Zahl der 119

Verteidiger $175,351,566,572,585,591,595$, $739,949,1066,1079,1085$

- jüdische Pflichtverteidiger s. auch Konsulenten, jüdische 128, 163, 180

- Stellung und Rechte im Verfahren 567, 570, 573, 585, 949, 960 f., 983, 985 f., 989, 992, 999 f., 1004 f., 1015, 1030, 1045, 1050 f., 1090

- Zulassung und Bestellung 180, $185 \mathrm{ff}$., 1004 f., 1014 f., 1069 f., 1075, 1089, 1136

Verwaltungsgerichte s. auch Oberverwaltungsgericht $537 \mathrm{ff}$., 544,556

Verwarnung mit Strafvorbehalt 777, 789, 985

Völkerbund 863

Völkische 47

"Völkischer Beobachter" 61, 64, 83, 87, 90 ff., $96,100,115,126,131,226,329,338,437$, $445,457,473,478,484,487,652,677$, 686 f., $710,724,731,755,758,767,795$, $799,832,877,896,898,903,923,958,982$, 1129 
Völkischer Block 19, 48

„Völkischer Kurier“ 45

Völkisch-sozialer Block 64

"Volksbote“ 375

„Volksgemeinschaft" 63, $65 \mathrm{f}$., 122, 131, 134, 217, 304, 475, 498, 616, 693, 713, 727 ff., $733,736,741,761,763,774,778,784,790$, $810,814,846,850,875,897,918,924,932$, 989, $992 \mathrm{f} ., 999 \mathrm{f} ., 1018,1025,1027,1064$, 1067, 1101, 1131

Volksgericht(e)

- bayerische $22 \mathrm{f} ., 26,35$

- München I 25, $28 \mathrm{f}$., $33 \mathrm{ff}$., 39, 41 f., $45 \mathrm{f}$., 48

Volksgerichtshof $2,6,64,86,119,121,142$, $185,208,210,217,233,259,263 \mathrm{f} ., 270 \mathrm{f}$., 290 f., 294, 449, 496, 521, 527, 571, $600 \mathrm{f}$., $614,618,678,687,847,855,893,900,910$, 923, 933, $936 \mathrm{f} ., 939,941,943,949,951 \mathrm{f}$., 973, 976, 979f., 993, 996, 1011, 1017, 1029, $1038 \mathrm{f} ., 1041,1044,1062,1064,1069$, 1071 ff., 1081, 1083, 1095, $1099 \mathrm{f.,} 1132 \mathrm{f}$., 1136

- als „Reichsstrafgericht“ $966 \mathrm{f}$.

- Besoldung der Richter und Staatsanwälte 966, 968, 971

- Besonderer Senat 970, 1011, 1072, 1075, $1077 \mathrm{f}$., $1081 \mathrm{f}$., 1085, $1136 \mathrm{f}$.

- Bestellung der Mitglieder 960 ff., 968 f., 1075, 1132

- ehrenamtliche Beisitzer 935f., 943, $961 \mathrm{f}$., 966, 968, 970f., 976, 1132

- Errichtung und Organisation 956, $959 \mathrm{ff}$.

- Etatisierung $966 \mathrm{ff}$.

- Präsident $600 \mathrm{ff}$., $962,965 \mathrm{f}$., $968 \mathrm{ff}$., 972 , 974, 1013, 1015, 1038, 1100, 1133, 1137

- Rechtsprechung 862, $964 \mathrm{f}$., $1132 \mathrm{f}$.

- Verfahrensvorschriften 847, 960f., 988, 1000, 1050, 1060, 1064, 1075, 1136

- Zahl der Richter 961, 964, 966, 968, 970

- Zahl der Todesurteile 900, 965

- Zahl der Verfahren 969

- Zuständigkeit 960, 969, 977, 979, 1132

Volksgesetzbuch der Deutschen $752 \mathrm{f}$., $929 \mathrm{f}$.

Volksschädling(e) 26, 464, 506, 587, 686, 693, 695, 843, 906 ff., 1131, 1141

Vorbeugungshaft $5,719-745,778,844,1024$, 1026,1028

- Erlaß des RMdI v. 14.12.1937 725 ff., 729, 733, 735 ff., 1029

- Erlaß Görings v. 13.11.1933 719 f., 726

- kriminalpolizeiliche 538,719ff., 1021, 1125

- politische s. Schutzhaft

- Versuch der Einschränkung $733 \mathrm{ff}$.

- Zahlen 721, 724f., 735, 738

- zur Korrektur der Justiz 729 ff., 733, 740 ff., $744,1027 \mathrm{f}$.
Vormund(schaft) 505 f., $511 \mathrm{f} ., 515,517,532$, 703, 772

„Vorschau“ s. unter Lenkung der Rechtsprechung

Voruntersuchung, gerichtliche $345,381,383$, 387,392 f., 395, 634ff., 638, 644, 649, 678, 791, 940, 958, 960, 981 f., 986, 991, 1001, $1005,1016,1050 \mathrm{ff} ., 1054,1063 \mathrm{f} ., 1096$, $1100,1132,1136$

Vorverfahren 1044, 1058

„Vorwärts“ 823

Vossische Zeitung $125 \mathrm{ff} ., 135,242$

„Waffe“ i. S. der Gewaltverbrecherverordnung 913, 1079 f., 1103

Wahlen s. unter Reichstag

Wahlfeststellung $773,852,855,861,984,989$, 1061, 1093, 1129, 1136, 1141

Waldenburg (Schlesien) 461, $464 \mathrm{ff}$.

Waldheim (Heilanstalt) 508,517

Wartestand 222

Wehrkraftzersetzung s. Zersetzung des Wehrwillens

Wehrmacht s. auch Reichswehr 68, 83, 267, $279,291,298,305,312$ f., 334, 500, 621, 649 f., 677 f., $786,813,815,817,819,832$, $855,873,886,892,920,938,945,974,980$, $1004,1017,1032,1093,1110,1142$

Wehrmachtgerichtsbarkeit s. auch Militärgerichtsbarkeit 412, 415, 654, 677 f., 854, 900, $907,911,915,1048,1072 \mathrm{ff}$.

Wehrmachtrechtsabteilung $1071 \mathrm{ff}$.

Wehrmachtstrafgesetzbuch s. Militärstrafgesetzbuch

Wehrmittelbeschädigung $776,820,855$, 886 ff., 890, 892, 919, 924, 969

Wehrpflicht 801 f., 854f., 863, 875, 992

Weiden (Oberpfalz) 491

Weimar 114

Weisungsfreiheit, richterliche s. Unabhängigkeit der Richter, sachliche

Weisungsgebundenheit der Staatsanwaltschaft 36,195 f., 452, 487, 540, 659, 729, 978, 986, $998,1004,1038,1041,1044,1057,1060$, $1077,1080,1085,1092,1095,1098,1101$, 1105 ff., 1121, 1134, 1142

„Westdeutscher Beobachter“ 856

Westerland (Sylt) 337

Widerstand 254

Wiederaufnahme des Verfahrens 26, 516,661, 714, 938 f., 948 f., 982 f., 987, 991 f., 1006 f., $1012,1038,1048,1059,1063,1066 \mathrm{f}$., $1071 \mathrm{ff}$., $1076 \mathrm{ff} ., 1135 \mathrm{ff}$.

Wien $174 \mathrm{f} ., 177,180,260,268,283,286,288$, 532, 596, 805, 807, 1017

Willensstrafrecht s. unter Strafrecht 
Wirtschaftsministerium, preußisches s. unter Preußen

Wirtschaftsordnung, kapitalistische 3, 665

Wirtschaftssabotage 894, 900, 969, 1095, 1131

Wirtschaftsstrafrecht $783,835,858$

Wirtschaftsverrat $781,783,796,818 \mathrm{f} ., 1052$, 1066

Wittenberg (Bezirk Halle) 566, 698

Wolfstein (Pfalz) 383

Württemberg $98,110,112,129,147,238$, 240,245 f., 259, 305, 513

Würzburg $56,698,766,826,1016$

Wuppertal 353 ff., 361, 704, 733, 965

Wyk auf Föhr 340

Zentralausschuß für die Innere Mission der Deutschen Evangelischen Kirche 506

Zentral-Justizamt für die Britische Zone 240

Zentralstaatsanwaltschaft 224, 324, 332, 345 bis 348,350 ff., 363 ff., $373,406, \mathbf{4 4 2 ,} 444 \mathrm{ff}$., 462, 480, 683, 712, $714 \mathrm{f}$., 717 f., 954, 1091, 1095, 1116, $1120 \mathrm{f}$.

Zentrum 9f., 49, 59, 144, 213, 222 ff., 242 f., 288, 461, 465, 863

"Zersetzung“ 17

- defaitistische 902

- der Polizei 824, 845

- der Reichswehr 824, 845, 965

- des Wehrwillens 762, 782, 901, 906, 1131

Zeugnisverweigerungsrecht 804, 881, $1031 \mathrm{f}$, 1053
- für Geistliche $1030 \mathrm{f}$.

Zigeuner $588 \mathrm{f} .$, 726, 785, 1096

Zinnowitz (Pommern) 1017

Zivilarbeiter

- „fremdvölkische“ 197, 597, 688f., $1126 \mathrm{f}$.

- polnische $583,597,679,688 \mathrm{ff} ., 718,1089$, 1126 f., 1137

- sowjetrussische 597, 679, 718, $1126 \mathrm{f}$.

Zivilgerichtsbarkeit 931f., 935, 967, 976, 1014, 1069, 1102 f., 1143

Zivilprozesse 694 f., 984

Zivilprozeßordnung (ZPO) 118, 124, 993, 1053

Zivilrecht s. auch Bürgerliches Recht 865, 925, 967

Züchtigungsrecht 787

Zurechnungsunfähigkeit $516 \mathrm{ff} ., \quad 772,774$, 777, 779, 841 ff., 918, 984, 992, 1025 f., 1057 f., 1065

Zwangspensionierung $132 \mathrm{f}$., $137 \mathrm{f} ., 155 \mathrm{ff}$., 159 ff., 165 ff., 169 ff., 191 ff., 195 ff., 201 ff., 222 ff., 230, 237, 242 f., 248, 378 f., 512, 662, 1118 f., 1142

Zwangsversetzung s. unter Justizbeamte und unter Richter

Zwangsverwalter 700, 703

- jüdische 128

Zweibrücken (Pfalz) 323

Zweikampf 786f., 819, 837, 846, 1093

Zwischenverfahren s. auch Eröffnungsbeschluß $987 \mathrm{f}$., $1033 \mathrm{f}$., 1050 